\title{
Online communities of practice and professional change: A three-tier view of the knowledge embedding process
}

\author{
By \\ Jocelyn Cranefield \\ A thesis submitted to Victoria University of Wellington \\ in fulfilment of the requirements for the degree of \\ Doctor of Philosophy in Information Systems
}

Victoria University of Wellington

2009 



\begin{abstract}
This interpretivist study, in the field of Information Systems, investigates the process of transformative professional change using a knowledge management lens. The goal of the research was to understand how online communities of practice (CoPs) facilitate the transfer and embedding of professional knowledge. It was guided by the question: How do online CoPs facilitate the transfer and embedding of professional knowledge?
\end{abstract}

This topic was of contemporary and strategic significance in New Zealand: The government had embarked on a strategy to transform teaching in NZ schools, aiming to leverage a major investment in IT infrastructure, using online CoPs to help embed a new paradigm of studentcentred, ICT-enriched learning at system level. There was, however, no research to suggest how this might occur. Despite the increasing use of online CoPs by organisations, and an expansion in the number of tools available for this purpose, there is little understanding of how online CoPs can facilitate knowledge transfer. The way in which knowledge embedding (deep transfer) occurs, and the role online CoPs may play in supporting this process, is particularly poorly understood. This is significant issue in this internet-rich era, when developing nations are aiming to cultivate knowledge economies.

I conducted the research using a case research strategy, qualitative methods and an inductive process of theory generation. The research case was a national professional development programme for schools, with five CoP subunits: Four were regionally based school cluster CoPs and one was a distributed blogging community. (Membership of this community overlapped with three of the cluster CoPs.) Based on my analysis of data, and on feedback from participants, I found that three complementary mechanisms were operating simultaneously, facilitating the embedding of knowledge at meso, micro and macro levels. The result of my study is a threelevel explanatory theory.

At the meso (school) level, knowledge embedding followed a six-stage cycle, with different activities and issues characterising each stage. Online CoPs played a different role at each stage. At the micro (individual) level, knowledge embedding was driven by teachers' crossings of multiple engagement spaces (communication contexts) in a polycontextual environment. Crossings drove personalisation and facilitated the linking of theory and practice, leading to deep individual understanding. At the macro level, the embedding of knowledge was driven by the brokering function of a middle layer community in a system of overlapping, tiered CoPs. Key roles were played by two kinds of knowledge brokers: connector-leaders and follower-feeders.

All three embedding-facilitating mechanisms promoted five fundamental knowledge embedding processes: focusing, persuading, aligning, adapting, and owning (developing ownership). 
I dedicate this thesis to my mother and father, Judith and Mervyn Cranefield, who encouraged me to value education and think about things.

\section{ACKNOWLEDGEMENTS}

I thank the Tertiary Education Commission for funding my research and associated conference travel through a Top Achiever Scholarship. Thanks to the Ministry of Education for supporting this project and for facilitating access to my participants; especially to Murray Brown and Nancy Groh of the e-learning unit.

I am most grateful to Professor Pak Yoong for encouraging me to take on this endeavour and enthusiastically guiding and supporting me through it; also to Professor Sid Huff, my co-supervisor, for his interest and critical insights. Thanks also to the other academic and administrative SIM staff at Victoria University who provided support.

My sincere thanks go to my research participants and their employers who granted me their time, hospitality, and provided access to valuable thinking and online records.

Special thanks to my family - Tom, Kathy and Alice, and cousin Jen - for supporting me in this endeavour, and to the friends who have provided me with support and companionship; especially Jan, Nishanie, Trish and Jackie.

My thanks to Jan for IT support, Rob Hill for assistance with transcribing interviews and Helen Widdicombe for help with formatting. 


\section{TABLE OF CONTENTS}

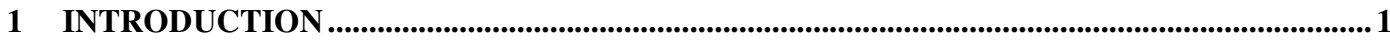

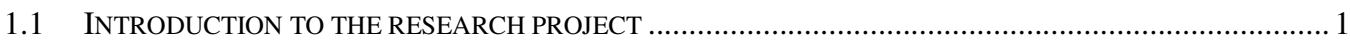

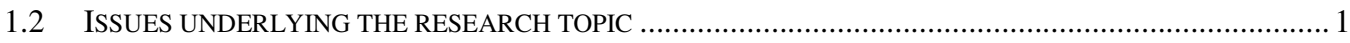

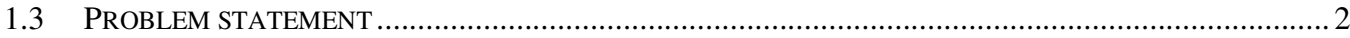

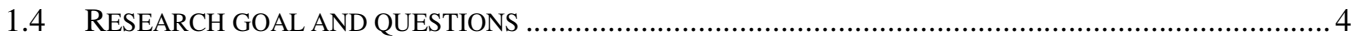

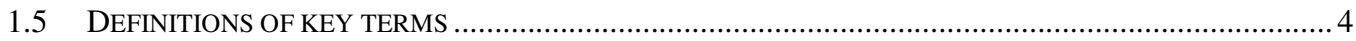

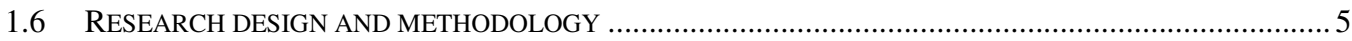

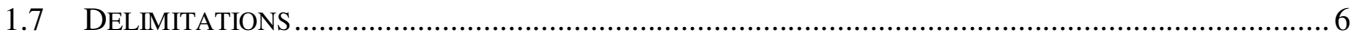

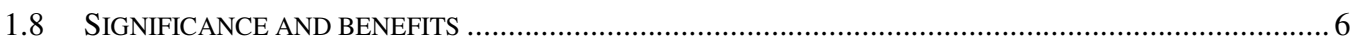

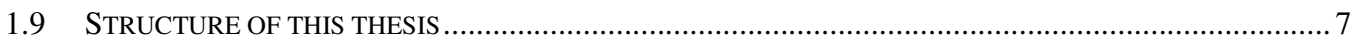

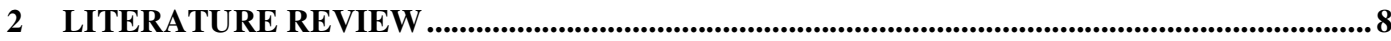

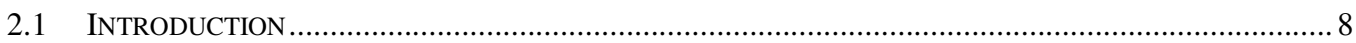

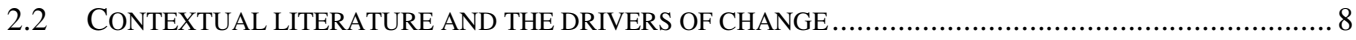

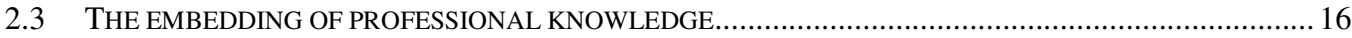

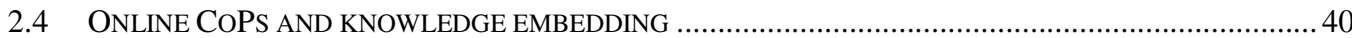

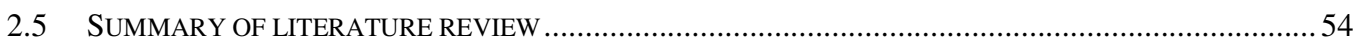

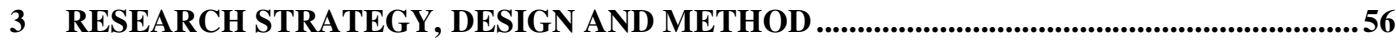

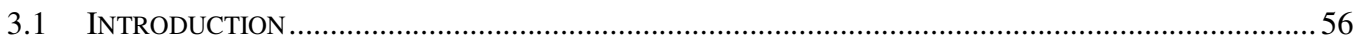

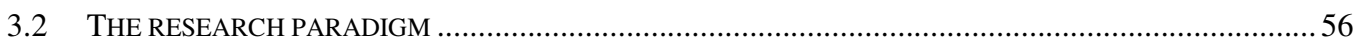

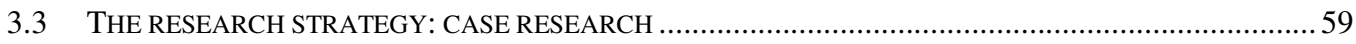

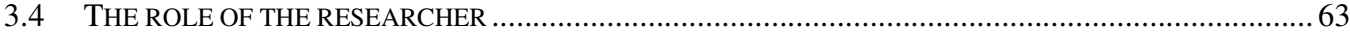

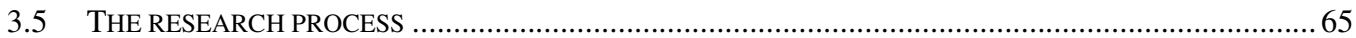

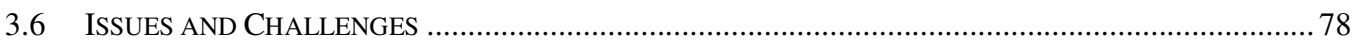

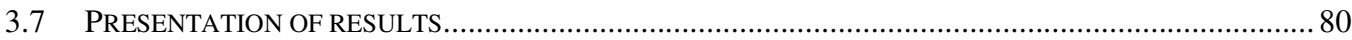

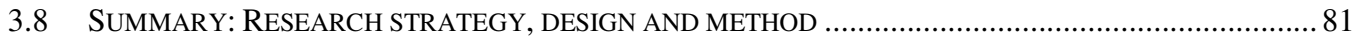

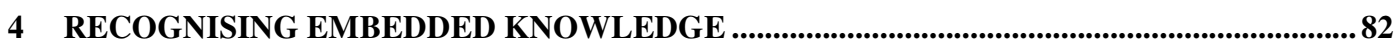

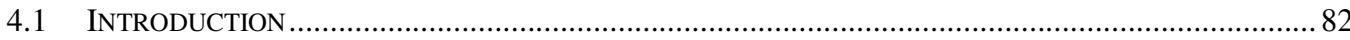

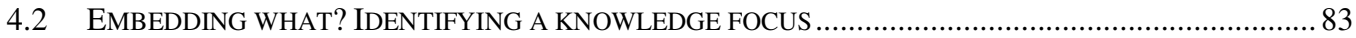

4.3 ELICITING INFORMATION ABOUT EMBEDDED KNOWLEDGE..................................................... 92

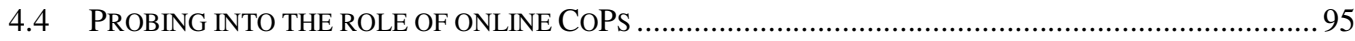

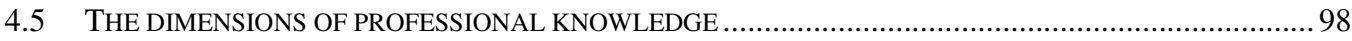

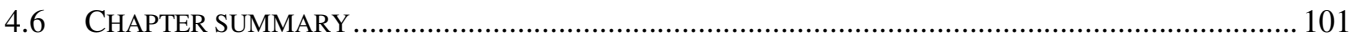

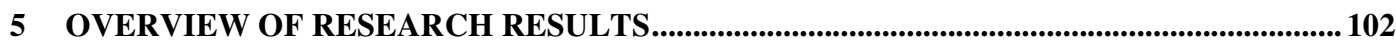

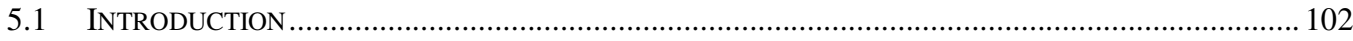

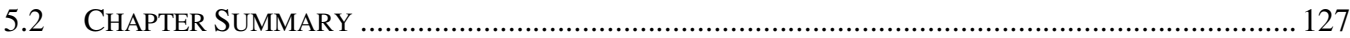

6 THE MESO-LEVEL KNOWLEDGE EMBEDDING CYCLE................................................... 128

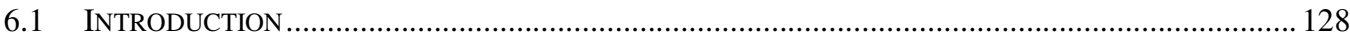

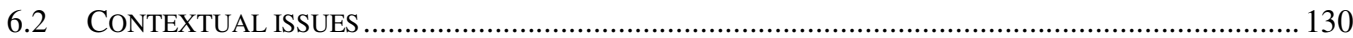

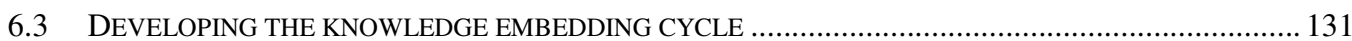

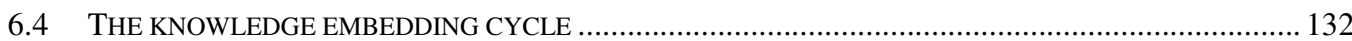

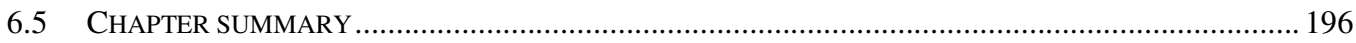

7 THE MICRO-LEVEL FACILITATING MECHANISM: CROSSINGS ................................... 198

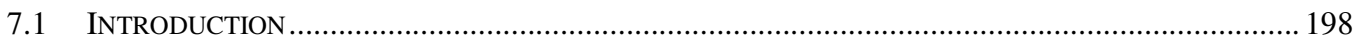

7.2 COMPLEX COP SYSTEM WITH MULTIPLE ENGAGEMENT SPACES …...................................... 199

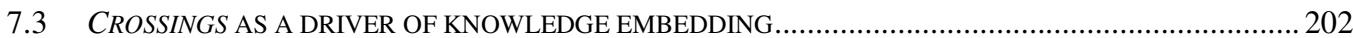

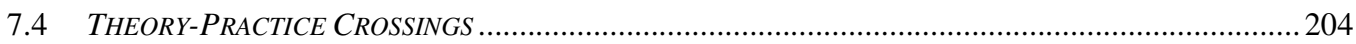

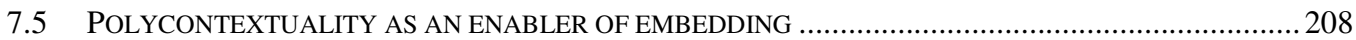

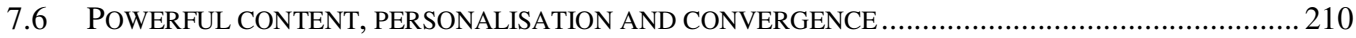

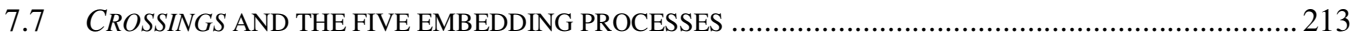

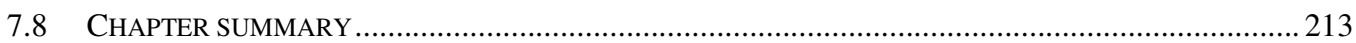


8 THE MACRO-LEVEL MECHANISM: INTER-COP BROKERING .....................................215

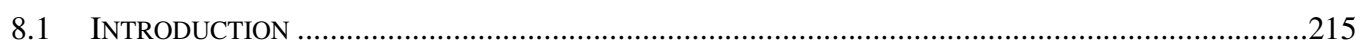

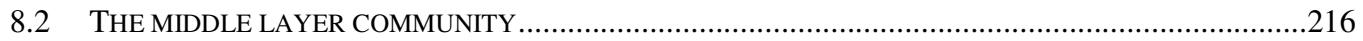

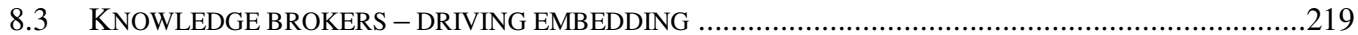

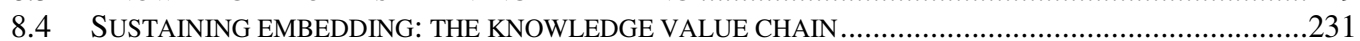

8.5 THE MACRO-LEVEL MECHANISM AND THE FIVE EMBEDDING PROCESSES ...................................234

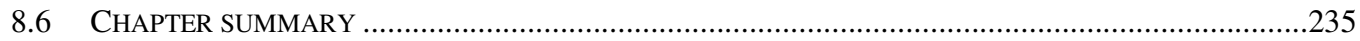

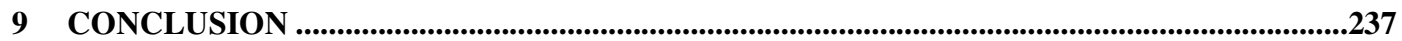

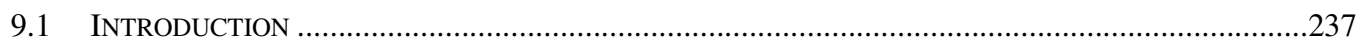

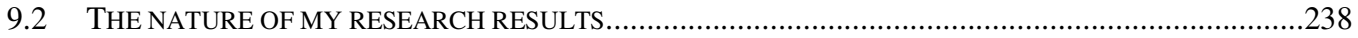

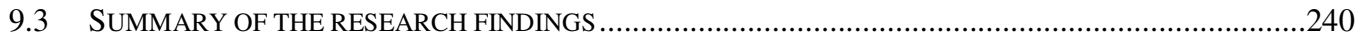

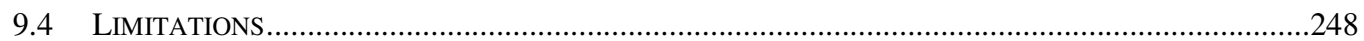

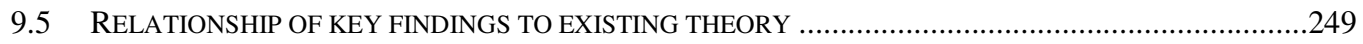

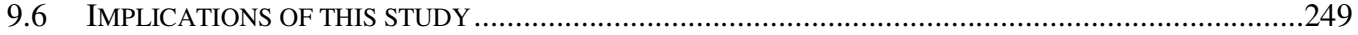

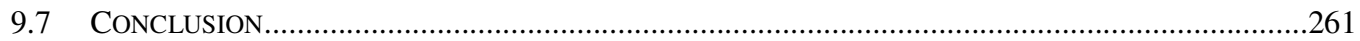

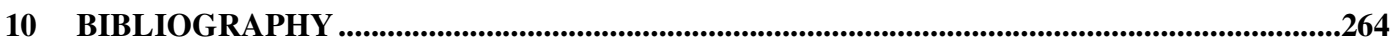

APPENDIX A

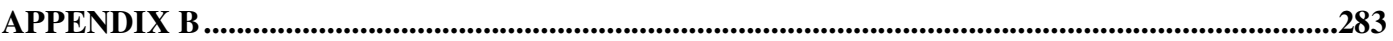

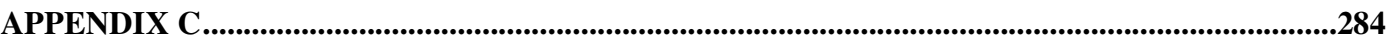

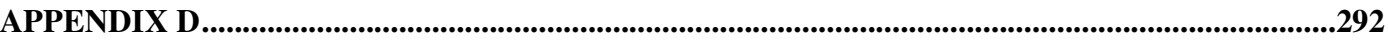

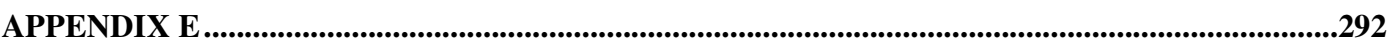

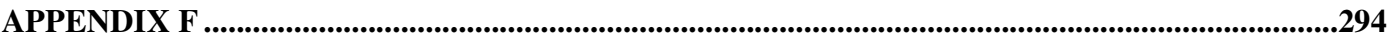

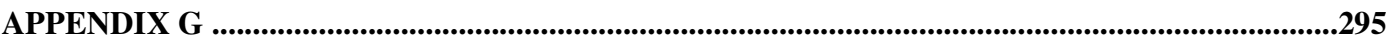

APPENDIX H 


\section{TABLE OF FIGURES}

Figure 1: PISA 2000: NZ's high variance in student outcomes ........................................................

Figure 2: Effective teaching and the three Vital Outcomes (MOE,2006c) ............................................... 10

Figure 3: The SECI model of the knowledge-creating company (Nonaka \& Konno, 1998) .................... 30

Figure 4: Castro's (2006) CoP conversation space ecosystem ......................................................5

Figure 5: The case structure showing overlapping CoP membership ..................................................63

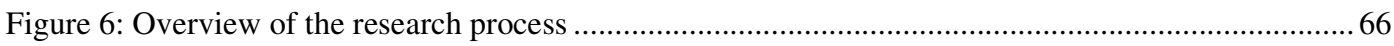

Figure 7: Data analysis process: interactive model (Huberman \& Miles, 1998, p.181) .........................70

Figure 8: The inductive mode of research in a qualitative study (Creswell, 1994) ............................... 74

Figure 9: The reflexive development of my "interpretive toolkit" ..................................................... 84

Figure 10: Establishing a Knowledge Focus: Comparison of top-down \& bottom-up themes ................. 89

Figure 11: Establishing a knowledge focus: the new way - four key themes....................................... 91

Figure 12: How clarification of a knowledge focus helped me to address the how question ...................91

Figure 13: Early exploratory map of professional knowledge (based on NVivo codes) ...........................99

Figure 14: Later working model of knowledge dimensions ........................................................... 101

Figure 15: Participating CoPs, showing overlapping membership with CoP E ................................ 116

Figure 16: Three-level theory: micro-, macro- and meso-level facilitating mechanisms ....................... 117

Figure 17: The six-stage knowledge embedding cycle ................................................................... 121

Figure 18: The macro level perspective - CoP E as a hub and middle layer community ...................... 124

Figure 19: Interaction of connector-leaders, follower-feeders and regular teachers .......................... 127

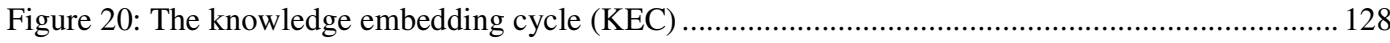

Figure 21: Knowledge Embedding Process - Iterations 1 and 2 ................................................. 131

Figure 22: Knowledge embedding cycle: final spiral-based model ................................................. 132

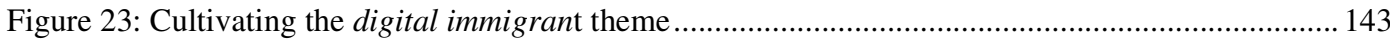

Figure 24: Cultivation of a theme from Prensky (2001) in online forum, ............................................ 144

Figure 25: Dimensions of knowledge being embedded .................................................................. 152

Figure 26: Photos posted in a forum by the facilitator to reward a contributor .................................... 160

Figure 27: Invisible e-mail to a connector-leader from a follower (CoP E) ........................................ 173

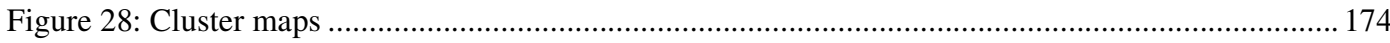

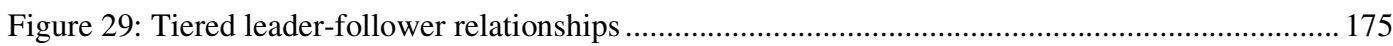

Figure 30: Polycontextuality - amplification of the professional environment ..................................200

Figure 31: Engagement spaces, showing points where crossings were made .................................... 202

Figure 32: The multiple dualities of context provided by the system of engagement spaces.................. 203

Figure 33: Making WebQuests practical -crossings between buddies............................................... 207

Figure 34: Embedding of memorable themes via crossings ........................................................ 211

Figure 35: Embedded themes compared with 'source' ....................................................................... 211

Figure 36: Tiered online CoP showing CoP E as a middle layer community .......................................2 217

Figure 37: Interactions between tiers of system participants in a subset of CoP B …......................... 231

Figure 38: Knowledge value chain: reciprocal flows that drove and sustained embedding ................... 233 


\section{TABLE OF TABLES}

Table 1: Effective Teaching Practices Using ICT (MOE, 2006B, p. 10)...............................................15

Table 2: Comparison of three staged-based models of KT ..............................................................27

Table 3: Processes and activities that facilitate types of knowing at Kappa (Orlikowski, 2002) ...............28

Table 4: Stages of CoP development: Comparison of six models, ......................................................47

Table 5:Data types and their perceived suitability for revealing embedding processes...........................68

Table 6: Examples of probing questions about the role of online CoPs in embedding knowledge ............97

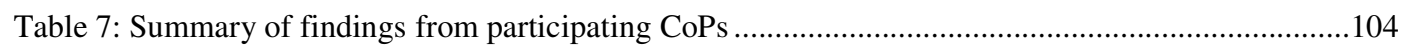

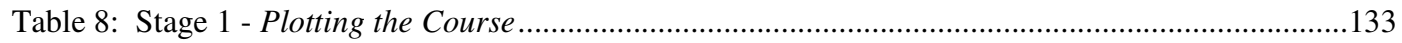

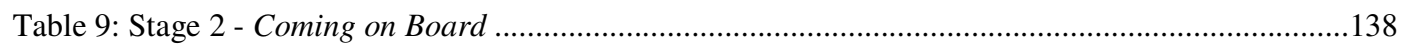

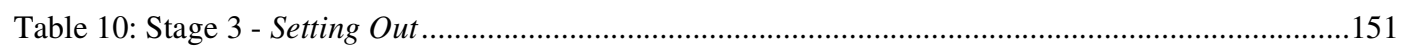

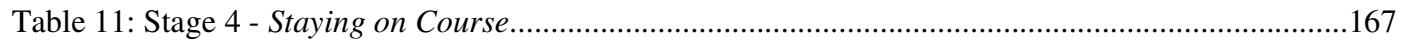

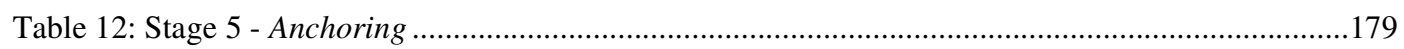

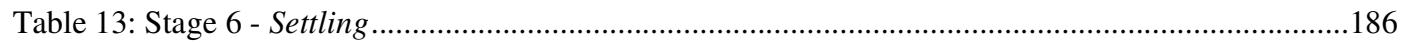

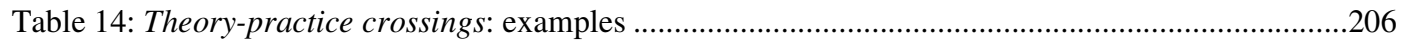

Table 15: The micro-level mechanism and the five knowledge embedding processes ...........................213

Table 16: Quotes from interviews indicating hierarchical CoP structure ............................................218

Table 17: Filtering and focusing practices of connector-leaders ......................................................223

Table 18: Reinforcing and contextualising practices of connector-leaders ............................................225

Table 19: Feeding and Helping others: practices of connector-leaders ...........................................227

Table 20: The macro-level mechanism and the five knowledge embedding processes..........................234

Table 21: Overview of how the meso-level theory answers the research question(s)............................243

Table 22: Overview of how the micro-level theory answers the research question(s) .........................244

Table 23: Overview of how the macro-level theory answers the research question(s)..........................247

Table 24: Relationship of key findings to existing theory ...............................................................250 


\section{Introduction}

At the heart of [educational] transformation are...online communities of educators who are passionate about transferred innovation.

(Hargreaves, 2003, p.66)

\subsection{Introduction to the research project}

This study was motivated by my recognition of the New Zealand (NZ) government's strategic dependence on online communities of practice (CoPs) to help drive a change initiative aimed at embedding professional knowledge across the schooling sector. This was both an unresearched area in Information Systems (IS) and an area of societal concern. In this chapter I outline the issues underpinning the study, set out the research goal and questions, and discuss the significance of this study.

\subsection{Issues underlying the research topic}

\subsubsection{Drivers of change in New Zealand's schooling system}

This study is underpinned by four interrelated issues that are driving change in NZ's schooling system in the early twenty-first century. The first issue is a lack of educational equity, reflected in the wide disparity of student outcomes in comparison to most developed countries (OECD, 2005). This has triggered a strategic drive for more effective, equitable teaching on the part of the Ministry of Education (MOE).

The second driver is the government's desire to integrate Information and Communications Technologies (ICT) into teaching, capitalising on its investment in ICT infrastructure. The effective use of ICT is seen as having potential to transform learning, acting as an equaliser:

e-Learning ${ }^{1}$ has the potential to transform the way we learn... [and] provide accessible, relevant and high-quality learning opportunities so...every student is better able to achieve their full potential. (MOE,2006a, p.4)

The third issue is an emphasis on using knowledge to drive changes in educational policy and practice (OECD2003). The MOE has invested in building knowledge about what works in education, based on a synthesis of research. It has signalled a need to

\footnotetext{
${ }^{1}$ The term e-learning refers to the use of ICT in school teaching and learning in a broad sense.
} 
embed this knowledge across the schooling system (MOE,2006c). This includes knowledge about effective teaching with ICT:

While there are many examples of highly effective practices using ICT in schools, these practices are not yet fully embedded into everyday teaching practice, both within and between schools. These changes in teaching practice are not yet systemic. The challenge now is to ensure that what we know about effective teaching and learning using ICT is rapidly spread and adopted throughout the school system.... (MOE,2006a, p.6)

The fourth driver of change is NZ's goal of becoming a knowledge society. A new view regarding the kind of knowledge that tomorrow's citizens will need has led to a reengineering of the school curriculum. The traditional subject-based approach is being replaced with a student-centred model, based around developing students' capacity for learning, or learning competencies. This is a paradigm shift.

Together, these four issues create a powerful agenda for educational transformation one that impacts powerfully on the professional role of the teacher. Of particular significance to this study, the proposed transformation is reliant on a strategy of community-based knowledge transfer (MOE,2006a, 2006c). According to the MOE's website, the potential of ICT to transform learning "relies on the education community sharing knowledge and understandings of the effective use of e-learning" (MOE2008).

Online CoPs are to play a key role:

[ICT can] facilitate and support educators to engage in creating, using, and sharing evidence within professional communities...

(MOE,2006a, p.17)

\subsection{Problem statement}

\subsubsection{The challenge of knowledge transfer}

NZ's government has embarked on a strategy for change. It relies on the nationwide transfer of knowledge between teachers and schools via CoPs, with an emphasis on the use of online CoPs. The knowledge at stake is professional knowledge - it concerns effective teaching, notably through the use of ICT, and a new student-centred paradigm. The goal is transformative change. However, knowledge transfer (KT) is known to be difficult, presenting multiple challenges (Gorgoglione, 2003; Harada, 2003; Szulanski, 2000). The problem of KT may be more daunting where knowledge must be transferred across time and space (Davenport \& Prusak, 1998; Sarker et al., 2005). The MOE's documents do not address this issue. Instead, its e-learning Action Plan 
uses language suggesting a Diffusion of Innovations (DOI) perspective (Rogers, 1962), according to which innovations are progressively adopted by members of a social system. Research into the transfer of evidence-based practice in health has shown that a DOI lens, which views knowledge as an innovation that is present or absent, is too simplistic for studying KT (Greenhalgh et al., 2005). Teaching is a similarly complex area of knowledge.

The challenge is compounded by the fact that, unlike the business sector, education has traditionally undertaken change from the centre. It has no history of using KT to drive improvement (Hargreaves, 2003). UK-based visionary Hargreaves sees it as necessary to change this:

There is a profound lesson to be learned in...business and industry, which have produced a culture of relentless innovation and the capacity to transfer it as the key to success. The education sector must now do the same. (2003, p.74)

The strategic imperative for KT in education is clear and the online infrastructure to support CoPs is available, but how do online CoPs drive KT? The ways in which online CoPs might facilitate knowledge embedding, the problems that may arise, and how these should be addressed, are unclear.

\subsubsection{Poor understanding of the knowledge embedding process}

The government's strategy aims to embed knowledge throughout the school system, but the way in which knowledge is embedded is poorly understood. There is no agreement as to what the knowledge embedding process constitutes, and a lack of research about how it can be facilitated. In addition, while the government's concern is embedding knowledge at a system level, KT studies are typically focused at an organisational or inter-organisational level.

\subsubsection{Lack of evidence about how online CoPs work}

The e-learning Action Plan (ibid) portrays online CoPs as playing a key role in the system-wide transfer and embedding of professional knowledge. Hargreaves paints a similar picture: "At the heart of transformation are communities of educators who are passionate about transferred innovation" (2003). He sees ICT as facilitating KT in "a small world where every teacher is linked by a short chain to the right peer" (ibid, p.14). Unfortunately, there is a lack of research to support this vision. As Hargreaves acknowledges, "we know too little about the dynamics of on-line communities, both in general as well as in education" (ibid, p.15). 


\subsubsection{Summary of the problem}

NZ's MOE has stated a need to drive change by embedding knowledge about effective teaching throughout the schooling system. Having invested in building a national IT infrastructure, it sees online CoPs as instrumental in this strategy. There is support for this strategy at a visionary level, but no research-based understanding of the knowledge embedding process or of how online CoPs might facilitate it.

\subsection{Research goal and questions}

The goal of my research was to gain an understanding of how online CoPs can facilitate the transfer and embedding of professional knowledge. I aimed to elucidate the nature of the knowledge embedding process and the activities involved, and to identify the technologies, roles and other factors that contribute to this process in the online CoP context. My research was guided by the overarching question:

- How do online communities of practice (CoPs) facilitate the transfer and embedding of professional knowledge?

and the subsidiary questions:

- What technologies, roles, and other factors help online CoPs to embed knowledge?

- What is the nature of the knowledge embedding process in online CoPs?

\subsection{Definitions of key terms}

Embedded knowledge is knowledge that is strongly contextualised (localised, customised, or personalised) and integrated with other knowledge. In colloquial terms, it has stuck or is sticky (Hippel, 1991; Szulanski, 1996); so well integrated it is difficult to un-stick and/or transfer.

Knowledge embedding is the process through which knowledge becomes embedded. In knowledge management $(\mathrm{KM})$ literature this process is seen as occurring either as the final stage of KT (Boisot, 1998; Kwan \& Cheung, 2006; Szulanski, 1996) or an ongoing dimension of KT (Sanchez, 2005).

Knowledge transfer $(\mathrm{KT})$ is a complex process, comprising various stages and/or activities, through which knowledge from one source is given to and taken up by another.

Note: Knowledge sharing does not necessarily result in KT, but this term is sometimes used interchangeably with the term KT. Use of the phrase "knowledge transfer and embedding" in my research question emphasises my focus on 'real' or deep knowledge transfer; knowledge that is both transferred and embedded.

Professional knowledge is knowledge that is profession-specific and is used by members of that profession to do, and think about, their work. 
A Community of Practice $(\mathrm{CoP})$ is a group of people who interact regularly and are united by a shared interest area or profession, and the value they place on learning in that area (Wenger, 1998b). CoPs are characterised by a sense of joint enterprise, shared accountability to a body of knowledge; relationships of mutual engagement; and a shared repertoire of communal resources; artefacts, assumptions, language, and understandings (Wenger et al., 2002).

An Online CoP relies on online tools to connect its members, who may also meet face-to-face (Dubé et al., 2006; Lai et al., 2005). Members may use traditional media (e.g., phone/fax) as well as IT tools (e.g., e-mail, videoconference, newsgroup, forum, instant messaging (IM), blogs, website, intranet) to interact. Traditional online CoPs are closed, facilitated and bound by a shared platform. Today, non-facilitated online CoPs may exist in dense areas of reciprocal links in blogging networks (Efimova \& Hendrik, 2005).

The terms micro, meso, and macro (derived from the ancient Greek mikros (small), mésos (middle), and macros (large) have been used in diverse ways to study the workings of complex systems in fields such as ecology, evolutionary science, the social sciences, and economics. Use of these terms reflects the view that there are multiple systems, or subsystems, simultaneously operating in a research setting, all of which play a part. I have used these terms as follows:

The micro-level perspective, or level of analysis, focuses on how the knowledge embedding process occurs in individuals who belong to a CoP and organisation.

The meso-level perspective focuses on how the knowledge embedding process occurs at the level of an organisation and its associated CoP.

The macro-level perspective takes a system-level view. It focuses on how knowledge embedding occurs between different CoPs (and their associated organisations).

\subsection{Research design and methodology}

My research set out to produce theory for explaining (Gregor, 2006) how online CoPs facilitate knowledge embedding. As is considered appropriate when generating explanatory theory, I approached my research question using case research strategy (Yin, 2003), studying a unique, real-world situation; the ICT Professional Development (ICT PD) programme for schools. According to Yin (ibid), case research is the most suitable approach when the researcher has little control over events, the focus is a contemporary phenomenon in a real-life context, and/or if how or why questions are asked. All three conditions were present in my study. The ICT PD programme provided a setting where teachers and schools were using online CoPs while moving to a new, student-centred, ICT-enabled teaching approach which I term the new way.

I adopted an interpretivist paradigm and used qualitative data gathering and analysis methods, for reasons outlined on pp.58-59. In generating theory, I took an inductive approach, as is considered suitable when there is little prior understanding (Rowlands, 2005). I outline and justify my research method and design in Chapter 3. 


\subsection{Delimitations}

This study was concerned with elucidating the process through which knowledge is transferred and embedded; focusing on how online CoPs can facilitate this. I did not aim to develop an instrument for measuring the quality or effectiveness of the knowledge embedding process, or to verify the extent to which new knowledge is embedded in practice. Although the education sector provided the context for my study, the study is not about educational improvement or teaching practice per se.

\subsection{Significance and benefits}

This section outlines the wider rationale for this research. I consider its relevance to the fields of Information Systems (IS) and Knowledge Management (KM), and its potential contribution to systemic change initiatives.

\subsubsection{Contribution to the IS field}

The state of knowledge in the IS field provided a strong justification for my research project. My study set out to help address the following issues:

\subsubsection{Understanding knowledge embedding}

$\mathrm{KT}$ is considered vital for organisational versatility, innovation, competitiveness, and survival (Earl \& Scott, 1998; Nonaka, 1998; Van Buren, 1999). In professional fields it is needed to keep understandings and practice current. Research suggests that KT is a complex process comprising different stages or sub-processes, with embedding being one of these. (Embedding is considered by some authors to be the final stage of KT while others see it as an ongoing aspect of KT.)

The embedding of knowledge is critical to the success of KT. Unless it is embedded, acquired knowledge can be seen as akin to a newly studied language that cannot be used in conversation. Despite this, knowledge embedding has received little attention from researchers. Argote et al. (2003) identify six KM topics into which further research is required. One of these is the embedding of knowledge in organisations.

\subsubsection{Enriching research into online CoPs}

This study seeks to enrich the research into online CoPs. Studies of online communities rarely go beyond identifying knowledge sharing to consider whether, and how, they have achieved the intended result $-\mathrm{KT}$ and embedding. My research 
focuses on the intersection of the topics of $\mathrm{KM}$ and online CoPs. It seeks to uncover the deeper workings of CoPs, so as to gain insight into their potential.

\subsubsection{Considering the use of social technologies for KM}

This study extends research into social technologies. Wagner and Bolloju (2005) note that, out of the available technologies used for $\mathrm{KM}$, the most popular are those that enable interaction in online communities. Exploration of social or collaborative technologies (such as wikis, blogs and collaborative publishing tools) is at an early stage. This project considers how these tools can support knowledge embedding.

\subsubsection{Extending research into the boundary spanner}

Previous research identifies the importance of boundary-spanning individuals in KT (Cranefield \& Yoong, 2007b; Harada, 2003; Pawlowski \& Robey, 2004). Boundary spanners perform multiple activities, filtering, interpreting and translating knowledge. This study extends this research by identifying the boundary-spanning roles and practices involved in facilitating knowledge embedding the context of online CoPs.

\subsubsection{Contribution to change-based initiatives at system level}

This study aims to contribute to understanding of strategic change initiatives by providing (a) a better understanding of the knowledge embedding process, (b) an understanding of how online CoPs can be managed to support this process at a system level, and (c) an appreciation of the barriers and enablers, including roles, practices and technologies, that are relevant to KT in this context. With the NZ government's emphasis on becoming a knowledge economy (IT Advisory Group, 1999) has come a growing awareness of the value of knowledge and KT.

\subsection{Structure of this thesis}

In the next chapter, I review the key research literature that is relevant to this study. Chapter 3 provides an account and justification of my research design and method. In Chapter 4, I recount how I focused my study around the embedding of a new teaching paradigm, which I call the new way. This positioned the research as being about high order professional change. Chapter 5 gives an overview of my results, which I outline in detail in Chapters 6-8. Chapter 9 outlines the significance, implications and limitations of my findings, suggesting areas for future research. 


\section{Literature Review}

\subsection{Introduction}

In this chapter I review literature of significance to the research topic, identifying key issues. I consider the relationship of my research to this literature and justify its importance.

In the first section, I outline the key contextual issues that impacted on this study. I also justify my selection of a professional knowledge lens, rather than a practice lens, for this study.

In the second section, I review research to do with KT and knowledge embedding, considering literature at three levels of analysis - organisation (meso-level), individual (micro-level) and system (macro-level). I identify a duality in the way in which the knowledge embedding process has been conceived. Finally, I consider the nature and limitations of micro-, meso- and macro-level theory and suggest that there is a need for a multi-level view, or theory, of how knowledge embedding occurs.

In the third section, I review research relating to the use of online CoPs to support KT and embedding. I consider a recent expansion of the definition of online CoPs to include dense areas of online networks (Efimova \& Hendrik, 2005) and the concept of an Online CoP Ecosystem (Castro, 2004, 2006). I also synthesise literature on the developmental stages of CoPs, and the roles and processes that might contribute to $\mathrm{KT}$ and embedding.

\subsection{Contextual literature and the drivers of change}

The context for my research was NZ's schooling sector at a time of system-wide change. As the result of public sector reform in the early twenty-first century, government agencies had moved away from their traditional outputs-based management approach to a new outcomes-based system. This had resulted in a new strategic focus and a greater level of social accountability. The emphasis in school education had fallen on the role of the teacher, with a goal of more equitable, effective teaching for NZ's diverse students. The MOE saw knowledge of what works in teaching as guiding this change. This knowledge needed to be embedded in the schooling system, with much of the onus for achieving this resting with professional communities. Online CoPs were to play a key role in driving this transformation. 


\subsubsection{Inequity and the drive for effective teaching}

The key driver of change underpinning this study was the government's discovery of a previously unknown level of inequity in education. A comparative international study, PISA 2000, had exposed the fact that, while NZ was a leading nation in terms of overall student achievement, it delivered a low level of educational equity to students compared with other developed countries (OECD, 2005, see Figure 1). Of specific concern, there was greater variation in achievement within schools than between schools. This was seen as indicative of a considerable variation in the standard of teaching. In other words, "Which teacher a student happens to get within a school matters more than which school a student happens to attend" (Nye et al., 2004, p.247).

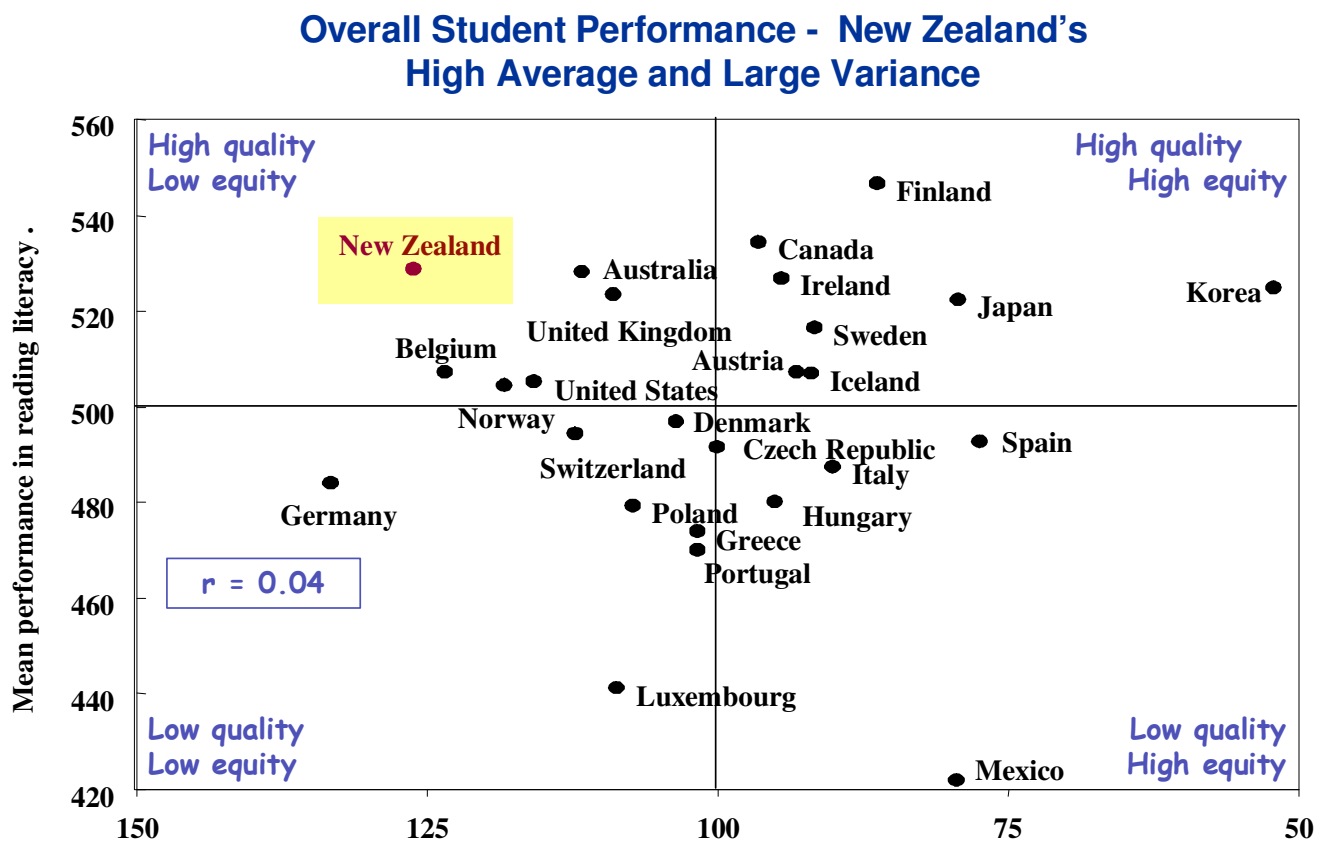

Figure 1: PISA 2000: NZ's high variance in student outcomes relative to other OECD countries. (MOE, 2005C)

The quality of teaching is the key determinant of student achievement (MOE, 2006c), explaining up to $60 \%$ of variation in student outcomes (MOE, 2004). In response to the inequity highlighted by PISA, the MOE identified effective teaching as one of three Vital Outcomes (see Figure 2), positioning it at the core of its Statement of Intent (2006c, p.12). The ICT Professional Development (ICT PD) programme for schools, which was my research case, sat within this initiative. It focused on the transfer and embedding of effective teaching and learning in the context of ICT use. 


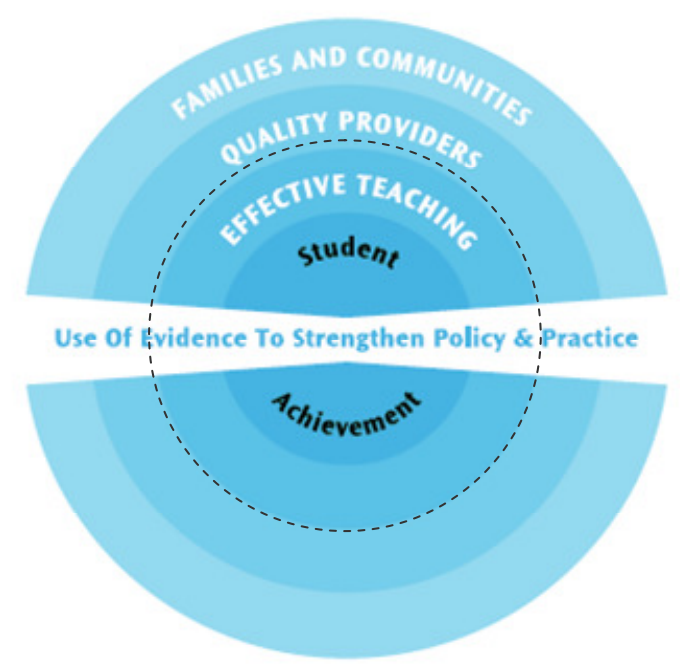

Figure 2: Effective teaching and the three Vital Outcomes (MOE,2006c)

\subsubsection{Knowledge as a strategic change agent}

The second issue underlying this research is a governmental emphasis on the use of knowledge to drive change in education. In 2006, the Secretary for Education said:

One of the...most important changes that I have seen is the huge growth in knowledge ${ }^{2} \ldots$ within our education system and profession. Evidence is now driving change...It is providing a knowledge base that is changing expectations, practice, and policy. (MOE, 2006b, p.2)

Knowledge is not only seen as the product of the Education system, but also as a key system input. A briefing to the incoming Minister (MOE, 2005a) advocated for the urgent embedding of knowledge of what is working. The objective was systemic change in the school sector:

There is an opportunity and an urgent need to learn from what is working. Practices need to be embedded across the system, in a sustainable way, to allow all students to benefit. This is a significant and important shift that will require concerted effort (ibid, p. 48).

\subsubsection{ICT as a driver of change in the teacher's role}

Another important theme is that ICT has been seen as a driver of change in the teacher's role. There are two sub-themes to this argument. Firstly, the use of ICT is seen as leading to changes in the way teachers teach, transforming their relationship

\footnotetext{
2 The new knowledge implicitly referred to is the Best Evidence Synthesis programme, a major synthesis of educational research sponsored by the MOE (Alton-Lee, 2006),
} 
with pupils into a partnership in which there is shared responsibility for learning (Gilbert, 2005; Hargreaves, 2003; Lai, 2005). Lai describes this as a paradigm shift:

Using technology not only lets teachers do things faster but requires a shift of paradigms in teaching and learning. It provides an opportunity to develop a totally new relationship between the teacher and the learner (ibid, p.15.)

Gilbert (2005) argues that as the use of ICT becomes more pervasive, the boundaries of the school will dissolve until the teacher becomes a learning broker (p.142).

The second way in which ICT is seen as changing the teacher's role arises from its networking potential (Cornu, 2004; Hargreaves, 2003; Ministry of Education, 2006a). ICT enables networked communication structures that are very different from the hierarchical structures of schools. Because networks have many nodes, and multiple pathways between nodes, they vastly increase the number of possible linkages, increasing access to like-minded colleagues, new ideas, and resources. In a seminal essay, Education Epidemic, UK-based educationalist, Hargreaves (2003) outlines a vision for transformation that capitalises on this networking potential. He promotes a strategy of lateral KT within education, arguing that the traditional approach, whereby government acts as a hub through which new policies are routed, is ineffective. He sees the key to transformation as innovation and $\mathrm{KT}$ via online communities and networks. A similar vision underlies NZ's e-learning Action Plan (MOE2006a):

ICT can...facilitate and support educators to engage in creating, using, and sharing evidence within professional communities...(p. 17)

In NZ's case, ICT seems to offer particular promise for building and supporting communities and networks of practice for teachers. Lai (2005) notes that while teaching has traditionally been an isolating job, in NZ this isolation is particularly pronounced: Nearly half the primary schools (46\%) have fewer than seven teachers (MOE,2005b). In the absence of ICT, this statistic makes for limited KT opportunities.

It is not only researchers and visionaries who view ICT as a driver of change in the teacher's role. A 2005 survey suggests that practitioners themselves view ICT as a transformative force. Johnson et al. (2005) measured principals' attitudes about the impact of ICT on teaching and learning. The majority of respondents agreed (or strongly agreed) that ICT is important to improving teacher practice ( $75 \%$ of primary and $80 \%$ of secondary schools) and that it makes a difference in teachers' and principals' professional practice (83\% and $86 \%)$. 


\subsubsection{Re-engineering schooling for the knowledge society}

As I embarked on my study, another high-level driver of change in schooling was exerting its influence. As part of the government's plan to transform NZ from a resource-based economy to a knowledge society (OECD,1999), a new concept of what kind of knowledge students should gain through their schooling was emerging.

In education, knowledge had traditionally been seen as stuff that resided in disciplines, being built up over time (Bereiter, 2002; Gilbert, 2005). However, as human understanding expands and as the future becomes less predictable, the model of knowledge as stuff becomes problematic. (Which stuff will be most relevant? For how long?) Today's increasingly complex, global society requires citizens who can learn and innovate continuously, solve problems and work with diversity, yet:

...it is an undeniable conclusion that the education system and its partners have failed to produce citizens who can contribute to and benefit from a world which offers enormous opportunity, and equally complex difficulty of finding your way in it. (Fullan, 2009, p.103)

Because of this concern, the emphasis of schooling in developing countries is shifting from building a stock of knowledge towards developing students' learning competencies and capacity for knowing (Bereiter, 2002; Fullan, 2009; Gilbert, 2005). While novel for education, this view of knowledge resonates strongly with that of KM theorists such as Snowden and Nonaka. For the schools and teachers in this study, it represented another point of transformative change.

Following a curriculum review in 2000-2002, a new competencies-based curriculum had been drafted. Following comprehensive consultation, it was moving to a final version at the time of my interviews. It significantly repositioned the emphasis of teaching, seeing students as being at the centre of teaching and learning (MOE,2007a) and coming with a call to personalise the student learning experience (MOE,2007b). These events were significant to my study because of the emerging curriculum's impact on, and synergy with, the ICT PD programme. The new competencies-based view of knowledge was an impending paradigm shift that was influencing programme facilitators and school leaders alike.

Schools were faced with a call to transform teaching, and ICT was seen as a means of helping to achieve this. Those who participated in the ICT PD programme had an opportunity to kick-start the transformation prior to the 2010 deadline for implementing a school-specific version of the new curriculum. The MOE's view of ICT's potential to 
drive transformation is hinted at in the e-learning Action Plan (MOE,2006a), published prior to the new curriculum:

For NZ, the development of a prosperous and confident knowledge society means the development of new skills and knowledge. It will require a culture of continuous enquiry, innovation and improvement, risk taking, and entrepreneurship. This can only come from the education system....We stand at the start of a new century, seeking to transform our nation. The power of education to drive that change is...potent... But we can only exercise that power if education itself is transformed, and this e-learning action plan will make a substantial contribution to that transformation. (MOE, ibid, foreword)

\subsubsection{Effective teaching}

This study investigates the transfer and embedding of knowledge about effective teaching, so it is necessary to consider literature relating to this theme. In the nineteenth century, teacher-training institutions were called normal schools because there was one accepted way to teach - the norm. Today, no single style of teaching is endorsed. Effective teaching is seen as a product of teachers' skill, understanding, imagination and resilience, and as depending on diverse factors, such as the age and ability of students, their backgrounds and needs, the subject and the resources available (Dunne \& Wragg, 1994). It is a complex professional activity that cannot be reduced to a single formula:

The teacher is continually making professional decisions, responding to instructional situations as a flexible problem-solver, and monitoring their students' progress. Effective teachers don't follow pre-determined programmes of...instruction. They align the....activities with their students' progressions (MOE,2003, p.79)

While research into best practice is widespread in other disciplines, educational discourse typically avoids using the term best practice. The concept is considered problematic because a practice that is well-suited to one context may not work at all in another (English \& Baker, 2006). The term effective practice is therefore used instead. It is difficult to recognise which practices are effective and suitable to transfer. According to Hargreaves (2003), "much that is said about good practice is based on mere opinion or unsubstantiated assertion rather than robust evidence about "what works" in particular circumstances" (p.46).

Based on a major synthesis of empirical research, the MOE has identified ten principles of effective teaching, (Alton-Lee, 2003). However, the way and extent to which any of these principles work in a given setting is seen as depending on the 
curriculum area, the teacher's experience, and the prior knowledge and needs of the learners. In addition, while the term effective practice implies a discrete teaching act, because of the diversity and the constantly changing needs of students, effectiveness requires teachers to focus on "maximising learning outcomes for all learners in every situation" (MOE,2006c). In other words, much of a teacher's effectiveness comes down to their circumstantial judgement and knowledge.

Rather than basing this study on the transfer of practice, I elected to use KT as the lens. This has several benefits: Firstly, professional knowledge is a richer construct than teaching practice. (Knowledge of teaching practice is only one of three types of knowledge that the MOE sees as contributing to effective teaching. The others are knowledge of subject and knowledge of students (MOE,2006c, p.29)). The construct of professional knowledge allows me to consider the breadth of what is being embedded, including how teachers think about teaching and what they value. Secondly, practice concerns what teachers do in their classrooms. I am not an educationalist and this is not an educational study, so I did not consider it appropriate to engage in observations of teaching. My data is based on professional communications and reflections; knowledge about practice. Thirdly, because the MOE itself emphasised the need for the embedding knowledge, a KT lens seemed appropriate. My knowledge focus aligns this study with a rich body of KM literature.

\subsubsection{Effective teaching with ICT}

Effective teaching with, or through, ICT is an emerging area of educational research. According to Ham (2005), there is no common view of what constitutes effective practice in this area, although the literature abounds with suggestions. The MOE's e-learning Action Plan identifies five generic effective practices that use ICT, which reflect the new student-centred paradigm (see Table 1). The same document emphasises, however, that "there is no simple prescription for how to teach effectively with ICT: It is the teacher's strategic use of instruction that makes the difference" (ibid, p.10).

Teaching with ICT is seen as a dynamic area in which the possibilities are expanding faster than the evidence of their impacts on learning. For this reason, the MOE promotes reflective practice and peer-to-peer knowledge sharing. It calls on online CoPs and networks to facilitate $\mathrm{KT}$ :

One of the easiest and most effective ways to communicate what we know about best practice in e-learning is through the use of ICT... 
...While there are many examples of highly effective practices using ICT in schools, these... are not yet fully embedded into everyday teaching....within and between schools...The challenge... is to ensure that what we know about effective teaching and learning using ICT is rapidly spread and adopted throughout the school system. (ibid, pp6-8)

\begin{tabular}{|l|l|}
\hline & $\mathbf{5}$ Effective Teaching Practices Using e-Learning (ICT) \\
\hline 1 & $\begin{array}{l}\text { Create new learning environments...allow students to explore and experiment, think critically, work } \\
\text { creatively, reflect and plan, use feedback and self-assessment and create new knowledge }\end{array}$ \\
\hline 2 & Use customised tools that aid preparation, programming, assessment, and reporting \\
\hline 3 & Customise learning experiences to recognise individual, cultural, and developmental differences \\
\hline 4 & $\begin{array}{l}\text { Enhance communication and collaboration to build partnerships beyond the classroom, expanding } \\
\text { the community of learners and enhancing the quality of learning }\end{array}$ \\
\hline 5 & $\begin{array}{l}\text { Create new education communities by increasing the modes of teaching and learning and the range } \\
\text { of people who can be involved }\end{array}$ \\
\hline
\end{tabular}

Table 1: Effective Teaching Practices Using ICT (MOE, 2006B, p. 10)

\subsubsection{Section summary: Contextual issues}

In this section I have summarised the key contextual issues underlying this study. The setting for my research was NZ's schooling sector at the start of a period of transformational change. The government's aim was to improve the equity of teaching for students. At the same time, it was embarking on changing the essential what and how of teaching through a new student-centred, competencies-based curriculum.

The MOE's change strategy relied, in part, on online CoPs to drive the system-wide embedding of knowledge about effective teaching, including teaching with ICT. ICT was seen as a transformative force, with potential to change the teacher's role, placing students at the centre of learning. However, in order to achieve its promise, ICT needed to be used effectively. Effective teaching with ICT cannot be reduced to a list of skills, being reliant on teachers' professional knowledge and judgement.

In the following sections of this chapter I situate my research topic within the relevant theoretical and research-based literature and justify its significance to the IS field. 


\subsection{The embedding of professional knowledge}

\subsubsection{Introduction}

In this section I review literature relating to professional knowledge and how it is embedded. I consider the concepts of professional knowledge and embedded knowledge then summarise the literature relating to the knowledge embedding process. My focus is on uncovering material that helps answer the questions: What is embedded knowledge?, why is it important? and how is knowledge embedded? Because my research context is bound up with system-level change, I consider research that operates at three levels of analysis: the organisation (meso-level), the individual (micro-level) and the system (macro-level).

\subsubsection{The research question}

At the heart of my research is a concern with understanding a specific aspect of the KT process: the embedding of knowledge. In the literature I have reviewed, the embedding of knowledge is considered to be either a sub-process or sub-stage of KT. I used the phrase transfer and embedding in my research question owing to the emergent state of knowledge about knowledge embedding and the lack of a commonly-accepted definition of embedded knowledge. My focus on knowledge embedding provides a clear orientation for studying the KT process.

In the simplest of terms, embedding of knowledge can be seen as the whole point of $\mathrm{KT}$. It is the process of making knowledge that has come from elsewhere stick. The intended outcome of $\mathrm{KT}$ is knowledge that is embedded, integrated with other existing knowledge, and used in a routine way. Despite agreement in the literature that embedded knowledge is of value, only a small quantity of research considers the knowledge embedding process in depth. The process of embedding of knowledge in organisations has been highlighted as a hot topic in $\mathrm{KM}$, into which research is needed (Argote et al., 2003). The process of embedding knowledge in individuals falls within the under-researched KM theme of personal knowledge management (Pauleen, 2009).

\subsubsection{The range of literature surveyed}

The majority of literature that considers KT and embedding is situated in the context of business. My research is concerned with the embedding of knowledge in a noncommercial organisational environment. However, as schools are organisations, this literature is somewhat relevant. In order to consider individual professional knowledge and how it is embedded, it is necessary to also consider literature from the fields of 
education and cognitive psychology. Another body of literature investigates the embedding from a system level. This lies within the fields of sociology, public health and education, and has been conducted using varying theoretical frameworks.

\subsubsection{The nature of knowledge}

Knowledge management (KM) emerged as a field of research in the 1980's (Davidson \& Voss, 2002; Wiig, 2000). It spans the disciplines of information systems, economics, organisational behaviour, psychology, strategic management and sociology (Argote et al., 2003). KM literature portrays knowledge as a valuable resource that cannot easily be captured, being based largely in physical and social contexts (Argote \& Ingram, 2000; Barney, 1991; Barney et al., 2001; Brown et al., 1989; Dunford, 2000; Spender \& Grant, 1996). The principal value of knowledge is seen as being in its usefulness, including its potential for enabling an organisation to be flexible, innovative and responsive. Davenport and Prusak (1998) encapsulate this view in their description of knowledge as:

a fluid mix of framed experience, values, contextual information, and expert insight that provides a framework for evaluating and incorporating new experiences and information (p.5).

\subsubsection{The dimensions of knowledge}

KM theory adopts two broad categories or dimensions of knowledge, established by Polanyi $(1958,1967)$ : explicit knowledge -knowledge that can be articulated or written, and tacit knowledge - knowledge that cannot be fully articulated or captured because it is bound up with practice or know-how (Greenhalgh et al., 2005; Nonaka, 1998). For example, skilled workers cannot explain all the protocols that underpin their performance (Reed \& DeFillippi, 1990) and people know more than they can tell (Polanyi, 1967). Tacit knowledge exists both at the level of the individual and in organisations (Madhaven \& Grover, 1998), although organisational know-how cannot exist independently from the minds of its members (Kogut \& Zander, 1992; Nelson \& Winter, 1982). Workers therefore store components of organisational routines, which are difficult to observe, analyse or describe, in a distributed procedural memory (Cohen \& Bacdayan, 1994). Organisations are seen as needing to transfer tacit knowledge through social acts of sharing (Nonaka, 1998; Nonaka \& Takaeuchi, 1995; Van Buren, 1999).

Some authors reject the idea of a taxonomy based around the duality of tacit and explicit knowledge, claiming that even documented knowledge has a tacit dimension 
(Brown \& Duguid, 2000; Orlikowski, 2002; Tsoukas, 1996). As Brown and Duguid (2001) have pointed out, Polanyi did not propose two types, but rather two dimensions of knowledge. Tacit and explicit knowledge are, at the very least, interdependent. Nonaka has said they are:

...mutually complementary entities. Without experience, we cannot understand. But unless we try to convert tacit knowledge to explicit knowledge, we cannot reflect upon and share it organisationally. (Scharmer, 2001, p.4)

Architects of any KT initiatives need to bear this in mind.

\subsubsection{Professional knowledge}

Individuals in many work environments rely on professional knowledge. This is the knowledge that underpins and governs their performance in a profession, such as engineering, project management, teaching, etc; and which they use to interpret and understand their work. For this project, I define professional knowledge as knowledge that is profession-specific and is used by members of that profession to do and think about their work. In cases where an organisation employs many staff from a single profession, such as schools or hospitals, professional knowledge is part of organisational knowledge.

Professional associations concern themselves with capturing bodies of professional knowledge, but at the individual level, professional knowledge looks very different: It is strongly activity-oriented, contextualised and personalised (Borko \& Putnam, 1996; Bromme \& Tillema, 1995; Connelly \& Clandinin, 1985; Elbaz, 1983; Tillema, 1995). The context for my research was the school education sector, so I now consider the nature of teachers' knowledge.

\subsubsection{Teachers' knowledge}

According to Munby et al (2001), the concept of teachers' knowledge dates from the early 1980's. Teachers' knowledge is known to be complex and multi-dimensional, and there is no agreement on how to portray it. Some researchers have taken a reductionist approach, identifying multiple sub-categories of teachers' knowledge, while others have developed constructs to emphasise its richness, personal nature, and contextual dependency. These include personal practical knowledge (Connelly \& Clandinin, 1985; Elbaz, 1983), images (Calderhead, 1988), knowledge in action (Schön, 1983), situated knowledge (Leinhardt, 1988), and event-structured knowledge (Carter \& Doyle, 1987; Borko \& Putnam, 1996). Given the contextual nature of 
knowledge and the different perspectives of researchers, it seems pragmatic to adopt the view, expounded by Borko and Putman (1996), that:

What teachers know and believe is completely intertwined, both among domains and within actions and context" (p.677).

Teachers' judgement, referred to in the last section, can be seen as an integral component of their professional knowledge.

The literature about teachers' knowledge is also distinguished by a dichotomy between an emphasis on theoretical knowledge, characterised by paradigmatic modes of thought, and a concern with practical knowledge, characterised by narrative modes of thought (Bruner, 1985; Grossman, 1995; Munby et al., 2001). These modes inform different researchers' perspectives and can be seen as representing reciprocal dimensions of teachers' knowledge. For example, Leinhardt et al. (1995) highlight the existence of dualities: Knowledge gained through practice tends to be procedural, specific and pragmatic; whereas the knowledge teachers gain in academic settings is more declarative, abstract and conceptual. Teachers develop personal professional knowledge by integrating these dualities (ibid).

\subsubsection{What is embedded knowledge?}

When KT is of strategic significance, the intended outcome is embedded knowledge: Unless new knowledge is embedded, it will be unevenly dispersed and/or applied in limited ways, leading to inconsistencies and isolated, temporary benefits. It is necessary to embed new professional knowledge in individuals and organisations and sectors in order to keep practice current, reflect changing governmental, environmental, and societal concerns, and respond to new understandings. Despite recurrence of the term embedded knowledge in $\mathrm{KM}$ literature, there is no consistency as to how it is described, making it necessary to synthesise a working definition.

\subsubsection{Embedded organisational knowledge}

The resource-based view of the firm holds that by continually embedding new knowledge in people, structures, routines, tools, products and culture, an organisation can stay flexible in an ever-changing environment (Argote \& Ingram, 2000; Barney, 1991; Dunford, 2000; Spender \& Grant, 1996). Despite this, there are widely varying definitions of what embedded knowledge actually is.

Armistead (1999) and Tuggle and Goldfinger (2004) describe embedded knowledge as being based in, and influencing, an organisation's operational processes. Lam (1997) notes that knowledge can be "stored 'organically' in team relationships" (p.979), while 
Madhavan and Grover (1998) see embedded knowledge as a specialised kind of human capital; "the potential for new knowledge (that) is embedded in the team and its interactions" (p.2). It can be transformed into embodied knowledge, in the form of new products, by combining individuals' knowledge through their interactions. Blackler (1999), who defines embedded knowledge as residing in systematic routines, differentiates it from embodied (action oriented) knowledge, encultured knowledge (based on shared understandings) and encoded knowledge (conveyed by signs and symbols). However, he notes that "it is a mistake to assume that embodied, embedded, embrained, encultured and encoded knowledge can sensibly be conceived as separate one from the other" (p.1032). This suggests that there is value in an inclusive definition.

What the above descriptions have in common is a view of the knowledge embedded in organisations as being highly tacit and context-specific; integrated into, and inseparable from, an organisation's structures, processes, culture, and social context, and distributed amongst its human and non-human components.

Embedded knowledge is also part of organisational memory (Argote \& Ophir, 2000; Walsh \& Ungston, 1991). In an account of how knowledge is embedded within hospitals, Ellingsen and Monteiro (ibid) describe it as being "moulded and crafted through re-presentations, thus creating a genealogy of historical sediments of layers of knowledge representations" (p.205). This definition introduces the idea that embedded knowledge may be incorporated into an organisation's documentation as well as its social structures and products. In other words, embedded knowledge may have an explicit as well as tacit dimension. Evidence to support this comes from several authors who view embedded knowledge as being stored in different kinds of organisational repositories (Badaracco, 1991; Kwan \& Cheung, 2006; McGrath \& Argote, 2001; Sanchez, 2005; Walsh \& Ungston, 1991). For example, Walsh and Ungston (ibid) propose a model whereby knowledge is embedded in individuals, culture, transformations (such as work design, procedures and administrative systems), organisational roles and structures, and workplace ecology; as well as in external archives (such as former employees, competitors, and government).

Szulanski (2000) highlights two further dimensions of embedded organisational knowledge (he uses the term integrated): It is both routinised and institutionalised (Goodman \& Steckler, 1989; Szulanski, 2000). Routinisation is the process whereby 
new practices "progressively lose their novelty and become part of the objective, takenfor-granted reality of the organization" (p.29).

Knowledge that is embedded in organisations has also been seen as a means of gaining alignment by facilitating convergence in the interpretive frameworks of employees. Sanchez (2005) describes how embedding organisational knowledge "in the form of an official, institutionalized interpretive framework...pressures individuals to adjust their personal interpretive frameworks to conform to the view of "reality" embedded in the institutionalized framework" (p.27). This has obvious implications in the context of change initiatives.

In answering the question "What is embedded knowledge?", I view the definitions summarised above as being complementary. Based on the synthesised literature, I suggest that, although embedded knowledge may have a strong tacit dimension, it is not synonymous with tacit knowledge. Rather, it is distinguished primarily by its high degree of contextualisation and its strong integration with an organisation's (or group's or individual's) other knowledge. I now briefly review the literature of embedded individual knowledge, before arriving at a working definition.

\subsubsection{Embedded individual knowledge}

Research in KM often focuses on the unit of the organisation, viewing individual and group knowledge as subsidiary. Personal KM is an under-researched area (Pauleen, 2009). In order to consider the special nature of personal, individual-level embedded knowledge, it is useful to consider research from the fields of education and cognitive science.

Like the knowledge that is embedded in organisations, knowledge embedded in individuals is strongly contextualised. Whereas in organisations it is customised, in individuals it is personalised. Research suggests that professional knowledge is embedded in people's interpretive frameworks, belief structures, work routines and practices (Richardson \& Placier, 2001; Tillema, 1995). Educational studies have indicated that with increasing work experience, teachers' knowledge becomes more embedded - more personalised (Kagan, 1990; Tillema, 1995), stabilised (Bennett, 1992) and fixed in nature.

This is a problem in the face of change: teachers' knowledge can develop into selfreinforcing structures that make professional change difficult (Kagan, 1990; Louden, 
1991; Richardson \& Placier, 2001; Tillema, 1995). Embedded meaning structures can limit an individual's response to new information, leading them to reject new ideas if they do not readily fit the existing schema. Where the goal of professional development is transformative change, new practices must therefore be underpinned by compatible interpretive frameworks and beliefs in order to become embedded in a sustainable way (Handal, 2004; Richardson \& Placier, 2001; Smith et al., 2005). Of particular relevance to this study, Handal (ibid) has noted that this is particularly important when ICT is being introduced:

It is indispensable that teachers' instructional beliefs match principles underlying current constructivist reform, particularly in regard to incorporating technology in the classroom, so the effective educational change can take place (p.1).

This poses an issue for change-orientated professional development programmes, underlining the relevance of the question, how is professional knowledge embedded?

\subsubsection{Embedded knowledge: a definition}

Based on a synthesis of the literature reviewed, I developed the following definition:

Embedded knowledge is knowledge that is strongly contextualised (localised, customised, or personalised) and integrated with other knowledge. In colloquial terms, it has stuck or is sticky (Hippel, 1991; Szulanski, 1996); so well integrated it is difficult to un-stick and/or transfer.

Related terms which signify the variety of ways in which knowledge may be embedded are: absorbed, embrained, encoded, encultured, institutionalised, integrated, internalised and routinised.

\subsubsection{How does knowledge become embedded?}

It is clear that embedded knowledge is seen as significant. The literature explores its theoretical nature and locus, its benefits and the issues associated with it. However, there is relatively little understanding of the process, or processes, through which knowledge becomes embedded. In a high-level review of the KM field, Argote et al (2003) identify six key emerging KM themes into which further research is required. One of these is the embedding of knowledge in organisations. In this section, I firstly synthesise research concerning the moderators of KT and embedding. I then consider the few sub-streams of research that have investigated the knowledge embedding process. 


\subsubsection{Moderators of knowledge embedding}

Prior to considering the knowledge embedding process, I review research that investigates moderators of the KT and embedding process. Various factors, conditions, activities and roles have been identified as potential moderators of KT and/or embedding. For example, based on Szulanski (2000), Sarker et al. (2005) present an overarching framework of five elements that may moderate KT: channel, message, context, recipient and source characteristics. In addition, it is difficult to know which moderators have relevance beyond their research setting. Furthermore, few studies relate KT moderators to specific aspects (sub-stages or sub-processes) of KT, including embedding.

I firstly summarise generic moderators of KT that are of potential relevance to knowledge embedding, then highlight stage-dependent moderators that have been found to impact on knowledge embedding specifically. (In section 2.4.2.2 I review research into the moderators of $\mathrm{KT}$ in online CoPs.)

\subsection{Transparency and absorptive capacity}

Two organisational moderators of KT are the transparency of an organisation - its openness to communication, and its absorptive capacity - its ability to recognise the value of knowledge, absorb and apply it; effectively the ability to embed knowledge (Rolland et al., 2003; Szulanski, 2000). In these studies absorptive capacity was facilitated by shared the cognitive bases (similar mental models) of individuals.

\subsection{Knowledge convertibility, translation and interpretation} Holden and Von Kortzfleisch (2004) introduce the concept of knowledge convertibility as a moderator of KT. This is a combination of the perceived usefulness of knowledge and the availability of experts to reveal its significance to others. A related finding is that translation and interpretation can facilitate KT (Cranefield \& Yoong, 2007b; Gorgoglione, 2003; Holden \& Von Kortzfleisch, 2004; Wenger, 1998b). In a study of inter-organisational KT (Cranefield \& Yoong, ibid.) participants reported needing to translate and interpret knowledge to create meaning and prevent ambiguity.

Translation involved presenting ideas in language or images their colleagues would understand, while interpretation involved creating practical, interesting examples in familiar contexts. The new knowledge had to be explained, exemplified, and modified or simplified. New terminology was then appropriated by organisations, and persistent repetition of new terms helped embed their meaning.

According to Gorgoglione (2003) two cognitive processes are involved in KT: the upstream act of codification (turning knowledge into language, models and images) 
and the downstream act of interpretation (understanding this codified knowledge). Both depend on the successful selection and organisation of information. This is affected by the people's cognitive characteristics, backgrounds, goals, values and beliefs.

Difficulties can arise in selecting the code, motivating people to share knowledge, making knowledge accessible, and interpreting the coded information. If the goal is training, consistency between cognitive systems is seen as beneficial. If the goal is innovation, different cognitive systems are considered useful.

\subsection{Disembedding and adapting interpretive frameworks}

In order for new knowledge to become embedded, it may be necessary to disembed or unlearn existing knowledge and practices (Malhotra, 2002; Szulanski, 2000). The more institutionalised a practice is, the harder it is to dismantle it, and therefore the higher the unlearning barrier (ibid). Embedding and disembedding are seen as occurring simultaneously in a reciprocal process of adaptation as existing practices are modified to accommodate new knowledge (Goodman \& Steckler, 1989; Kwan \& Cheung, 2006). Sanchez (2005) found that in group settings, individuals are likely to seek cognitive congruence with their peers, adopting similar patterns of thought.

\subsection{Stage-dependent moderators}

According to the stage-based KT literature (summarised in section 2.3.9) the moderators of KT may have differing levels of influence at different stages of the process (Chai, et al., 2003; Cranefield \& Yoong, 2006; Kwan \& Cheung, 2006). For example, Chai et al (2003) found that mechanisms with high richness (the ability to communicate variety and depth of information) were most effective for the later stages of KT (embedding). Based on a review of 20 studies, Kwan and Cheung (ibid) align their stage-based model of KT with success determinants. Key determinants impacting on the final stages of KT (where knowledge embedding occurs) are: absorptive capacity, transmission channels, partner relations and reliability of source (Implementation stage) and causal ambiguity (Retention stage). Causal ambiguity is a difficultly in determining the cause of success or failure in replicating a capability. These findings show the value of taking a granular approach in KT research investigating specific stages or sub-processes, and illustrate the value of an awareness of stages in organisational-level KT.

\subsection{Gatekeepers, knowledge brokers and boundary spanners} A subset of KM literature considers the role of a key person (knowledge broker, gatekeeper, boundary spanner, champion, or promoter) in KT and innovation. A 
gatekeeper is a person who is exposed to, monitors and filters, external information for its relevance to an organisation (Allen, 1967; Katz \& Tushman, 1981). The gatekeeper establishes external networks and helps bridge terminological cultures and values systems (Hernandez et al., 2004). Gatekeepers and others who connect different organisations, communities, groups, or networks are known as boundary spanners (Allen, 1977; Cross \& Prusak, 2002; Tushman, 1977). These people may act as knowledge brokers, transferring knowledge across the boundaries they span by identifying opportunities, promoting ideas, facilitating their uptake, and recombining and adapting them to fit the recipient context (Brown \& Duguid, 1998; Davenport \& Prusak, 1998; Harragon \& Sutton, 1997; Wenger, 1998b).

Being a knowledge broker is a complex, multi-dimensional role, requiring a mixture of judgement, communication and relationship management skills. These include gatekeeping (Allen, 1967; Ancona \& Caldwell, 1992; Cranefield \& Yoong, 2007a; Katz \& Tushman, 1981), translating and interpreting - converting knowledge to fit the recipient context, increasing absorptive capacity (Cranefield \& Yoong, 2007b; Holden \& Von Kortzfleisch, 2004; Pawlowski \& Robey, 2004; Wenger, 1998b); and the ability to align perspectives (Wenger, 1998b). Despite the complexity of their work and the range of expertise required, knowledge brokers typically perform non- or semi-official roles and are accorded low institutional recognition (Tushman, 1977; Wenger et al., 2002). Organisations with silo-based structures are poorly equipped to support them, making it difficult to recognise their value of such roles, and knowledge brokers may experience feelings of inadequacy, arising from their sense of uprootedness; being "neither in nor out" (Wenger, 1998b, p.110). The importance of brokering is also a theme in the CoP literature.

I now consider research about how knowledge is embedded, reviewing literature that operates at several levels of analysis. Firstly I consider the embedding of knowledge in organisations, based on the KM literature. I then review research about how professional knowledge is embedded at the individual level, with reference to research in education and cognitive psychology. Finally, I consider research from diverse social sciences into the embedding of knowledge at system level.

\subsubsection{Embedding knowledge in organisations}

$\mathrm{KM}$ literature positions the knowledge embedding process as part of an overarching process of KT or knowledge creation. There are two sub-streams in this research: The first portrays KT as a linear change process comprising successive stages. Embedding 
(or an analogous construct) is seen as occurring in the final stage (or stages) of this process (Boisot, 1998; Kwan \& Cheung, 2006; Szulanski, 1996). The second substream views embedding as part of a continual process of knowledge creation (Nonaka, 1998; Nonaka \& Takaeuchi, 1995), knowing in practice (Orlikowski, 2002), or organisational learning (Argyris \& Schön, 1978; Sanchez, 2005). This apparent contradiction can be seen as arising from a duality of perspectives. Research that positions KT as a linear process focuses on tracking the transfer of a component of knowledge over time. Its aim is to understand the change process with the goal of helping managers predict the interventions needed at each stage. In describing how embedding occurs, this research concerns itself primarily with identifying what happens and when. On the other hand, the literature presenting KT as a continuous process operates at a deeper level. Its aim is to understand the nature of the ongoing mechanisms that foster KT and drive change. A concern with how the process occurs is uppermost. In the context of transformative change, both perspectives may be necessary to explain how professional knowledge is embedded. I now summarise these two sets of literature.

\subsection{Embedding as the final stage of $K T$}

Several studies portray KT as a structured process that occurs in discrete stages, each with its own activities and challenges (Boisot, 1998; Cranefield \& Yoong, 2006; Kwan \& Cheung, 2006; Major \& Cordey-Hayes, 2001; Szulanski, 1996). The value of such models is that they provide frameworks to help managers understand the KT process and to plan accordingly; anticipating the issues that are likely to occur. Different stages of KT may require diametrically opposed skills, so one must understand the issues specific to each stage (Kayes et al., 2005).

Table 2 compares three of these models: Szulanski (1996), Boisot (1998) and Kwan and Cheung (2006). While each positions knowledge embedding as occurring at the final stage - integration, impacting and retention respectively, they provide differing perspectives, with Szulanski's and Boisot's models focusing on routinisation and integration, and Kwan and Cheung's emphasising adaptation and retention of knowledge. On closer examination, embedding can be seen as beginning in the penultimate stage. In all three cases - ramp-up, absorption, and implementation - the activities describe the progressive application of knowledge in practice. This is reminiscent of Orlikowski's (2002) continual process view (outlined on page 30) and 
suggests that embedding should be seen not only a stage of KT, but also as an ongoing process.

\begin{tabular}{|c|c|c|}
\hline Szulanski (1996) & Boisot (1998) & Kwan \& Cheung (2006) \\
\hline \multirow[t]{2}{*}{ 1. Initiation } & 1. Scanning & 1. Motivation \\
\hline & 2. Problem-solving & 2. Matching \\
\hline \multirow{2}{*}{$\begin{array}{l}\text { 2. Implementation } \\
\qquad \quad \text { Knowledge is put to use }\end{array}$} & 3. Abstraction & \multirow{3}{*}{$\begin{array}{l}\text { 3. Implementation } \\
\text { - } \text { Knowledge is put to use } \\
\text { - } \text { Social ties established } \\
\text { - } \\
\text { Recipient uses } \\
\text { knowledge iteratively \& } \\
\text { performance is built up }\end{array}$} \\
\hline & 4. Diffusion & \\
\hline $\begin{array}{l}\text { 3. Ramp-Up } \\
\begin{array}{l}\text { - Gradual improvement } \\
\text { of performance }\end{array}\end{array}$ & $\begin{array}{l}\text { 5. Absorption } \\
\begin{aligned} \text { - } & \text { Knowledge applied } \\
& \text { to various } \\
& \text { experiences, } \\
& \text { resulting in learning } \\
\text { - } & \text { Knowledge } \\
& \text { becomes uncodified }\end{aligned}\end{array}$ & \\
\hline $\begin{array}{l}\text { 4. Integration } \\
\text { - } \quad \text { Routinization } \\
\text { - Shared history, } \\
\quad \text { meanings \& behaviours }\end{array}$ & $\begin{array}{l}\text { 6. Impacting } \\
\text { Knowledge is } \\
\text { embedded into } \\
\text { practices, artefacts, } \\
\text { rules \& behaviours }\end{array}$ & $\begin{array}{l}\text { 4. Retention } \\
\begin{array}{l}\text { - Institutionalisation } \\
\text { - } \\
\quad \text { Retention in a } \\
\text { repository }\end{array}\end{array}$ \\
\hline
\end{tabular}

Table 2: Comparison of three staged-based models of KT

\subsection{Embedding as a continuous process}

Another small set of KM literature presents an alterative view, seeing the embedding of organisational knowledge as a continual process (Armistead, 1999; Nonaka \& Takaeuchi, 1995; Orlikowski, 2002; Sanchez, 2005). I consider three of these studies, showing how they provide a useful complementary perspective.

Sanchez's (2005) model for embedding is based on an organisational learning framework. Five continuous learning cycles drive the emergence of new knowledge and its embedding into an organisation's interpretive framework(s), culture, systems, and processes. There is a movement from individual to group to organisation as knowledge emerges, and back again from organisation to group to individual as it is embedded. The learning cycles are (1) the individual learning cycle, (2) the individual/group learning cycle, (3) the group learning cycle, (4) the group/organisational learning cycle, and (5) the organisational learning cycle. Emergence occurs from cycle 1 to cycle 5, and embedding in the reverse direction. This model is of interest because it takes in account different levels of knowledge embedding in organisations and because its structural basis is the boundaries across which embedding occurs. A limitation is that it presents embedding as unidirectional. It 
can be argued that embedding may also begin with the individual or group and move to the organisation.

In a study of KT in a software development company, Orlikowski (2002) identified a repertoire of practices, activities and knowing that facilitated knowing how to do product development, leading to collective competence (p.257). The practices that helped embed different types of knowing were sharing identity, interacting face to face, aligning effort, learning by doing, and supporting participation (see Table 3).

Although these practices and activities may not apply to other contexts, Orlikowski's study is significant in relationship to my research for two reasons: Firstly, it portrays the richness and complexity of collective knowledge needed by specialist workers in an organisation, showing how different activities may be necessary to embed different aspects of knowledge (Orlikowski uses the term knowing to emphasise the fluidity and elusiveness of knowledge). Secondly, the study presents compelling evidence that the embedding of professional knowledge is a continual process.

\begin{tabular}{|l|l|l|}
\hline Practice & Activities & Type of Knowing \\
\hline Sharing identity & $\begin{array}{l}\text { Common training \& socialization } \\
\text { Using common orientation for development } \\
\text { work } \\
\text { Identifying with the organization }\end{array}$ & Knowing the organization \\
\hline $\begin{array}{l}\text { Interacting face-to- } \\
\text { face }\end{array}$ & $\begin{array}{l}\text { Gaining trust, respect, credibility, and } \\
\text { commitment } \\
\text { Sharing information } \\
\text { Building \& sustaining social networks }\end{array}$ & $\begin{array}{l}\text { Knowing the players in the } \\
\text { game }\end{array}$ \\
\hline Aligning effort & $\begin{array}{l}\text { Using common model, methods, metrics } \\
\text { Contracting for expertise annually } \\
\text { Using standard metrics }\end{array}$ & $\begin{array}{l}\text { Knowing how to co- } \\
\text { ordinate across time and } \\
\text { space }\end{array}$ \\
\hline Learning by doing & $\begin{array}{l}\text { Investing in individual development } \\
\text { Mentoring employees } \\
\text { Rewarding not punishing effort }\end{array}$ & $\begin{array}{l}\text { Knowing how to develop } \\
\text { capabilities }\end{array}$ \\
\hline $\begin{array}{l}\text { Supporting } \\
\text { participation }\end{array}$ & $\begin{array}{l}\text { Globally distributing product development work } \\
\text { Involving participants in project decisions } \\
\text { Initiating and supporting overseas assignments }\end{array}$ & Knowing how to innovate \\
\hline
\end{tabular}

Table 3: Processes and activities that facilitate types of knowing at Kappa (Orlikowski, 2002)

A critic of the resource-based view of knowledge, Orlikowski (2002) argues that knowledge cannot be a "separate entity, static property, or stable disposition embedded in practice" (p.250). She sees knowledge as enacted in practice on a dayto-day basis and uses the term knowing in practice to emphasise the fluidity of this concept. Knowing is "...an ongoing social accomplishment, constituted and 
reconstituted as actors engage the world in practice" (p.245). In other words, knowing is continually being embedded through workplace practices.

A similar view is provided by Nonaka and Takauechi's (1995) well-known SECI model for the knowledge-creating company. It shows knowledge as being continually created through interactions between explicit and tacit knowledge, based around four concurrent conversion processes: Socialisation, the sharing of tacit knowledge through peer-to-peer interaction; Externalisation, the articulation of tacit knowledge; Combination, the integration and dissemination of explicit knowledge; and Internalisation, the conversion of explicit knowledge into tacit knowledge by individuals (Nonaka \& Konno, 1998).

Although embedding does not explicitly appear in the SECI model, some inferences about it can be made. Given the stickiness of embedded knowledge, it appears that internalisation and socialisation - which generate tacit knowledge - are important contributors to knowledge embedding. However, these processes cannot occur without the other two. The embedding of knowledge must therefore be seen as arising from the whole SECI process. The central clockwise spiral indicates how this continuous process moves up through levels of the organisation. As the spiral expands, knowledge becomes more deeply embedded. This is a powerful, although complex theoretical model. Its strengths lie in its holistic nature and the structure it provides for analysing and managing complementary processes. Like Sanchez's model, it accounts for KT through interactions between individuals, groups and the organisation. A drawback of the model is that it was developed in the context of companies where there is extremely low staff turnover.

Like Orlikowski, Nonaka and his co-authors have highlighted the complexity of organisational knowledge and its strong social dimension. Once again, continually operating sub-processes can be seen as responsible for the embedding process. The views of Orlikowski and Nonaka - in which knowledge can never captured, but must instead continually be recreated or re-embedded, provide an answer to the question "How is knowledge embedded in organisations?" that complements the answer provided by the staged models. 


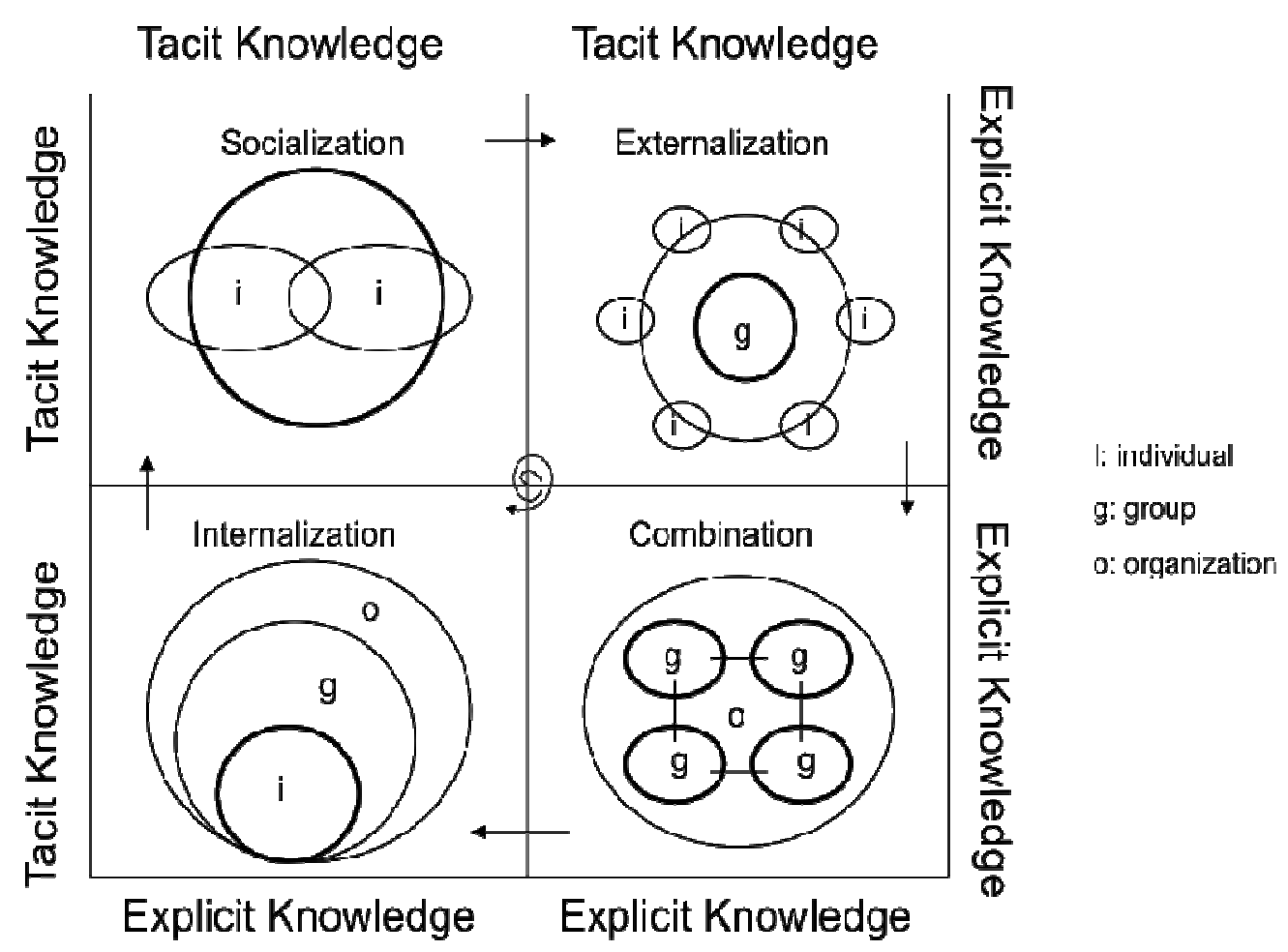

Figure 3: The SECI model of the knowledge-creating company (Nonaka \& Konno, 1998)

\subsubsection{Embedding knowledge in individuals (a micro-level view)}

There is no consensus as to how professional knowledge becomes embedded in individuals (personalised and integrated with other knowledge). However, research into how professional knowledge and teachers' knowledge develops, and how teacher change occurs provides useful insights, highlighting the likely challenges.

Bromme and Tillema (1995) note that although professionals are expected to draw upon a body of professional knowledge that is constantly being renewed, it is their performance in practice that generates (personal) professional knowledge (or knowledge in action (Schön, 1983). The professional therefore exists in a field of tension between professional action and theoretical knowledge. They must transform theory, integrating, tuning and restructuring it to the demands of practical situations and constraints (Bromme \& Tillema, ibid, p.262).

Leinhardt et al. (1995) view the development of teachers' knowledge as a recursive process, based around the integration of dualities of knowledge gained in practice (which is typically procedural, specific and pragmatic) versus knowledge gained in academic settings (which is more declarative, abstract and conceptual; pp 402-3). The 
degree of integration needed to develop skilful practice cannot result from the unidirectional translation of theory into practice. It requires the reciprocal transformation of knowledge learned in each of these settings into forms that are associated with the other. In other words, individuals must continually particularise theories and abstract theories from practice (p.403), making iterative, reciprocal readjustments.

Unfortunately, features of the workplace create barriers to this level of integration; in particular, the lack of opportunity for reflective and analytical thought. Leinhardt et al call upon the university education system to "create structures to slow down time" so as to facilitate reflective revision (ibid, p.404). (In 1995 it would have been impossible to consider whether online CoPs may help fulfil this function.)

While research on teachers' knowledge focuses on the emergence, establishment and growth of professional competence, research into teacher change deals with how to revise this knowledge once it has become embedded. This is strongly relevance to change programmes. In the case of teaching, research indicates that new knowledge cannot be easily 'added' because an individual's existing interpretive structures, attitudes and beliefs act as powerful mediators, filtering incoming knowledge (Handal, 2004; Tillema, 1995). Individuals have been found to ignore, reject or abandon information that does not fit their existing interpretive schemas (ibid, Sanchez, 2005) . For new knowledge to be accommodated, it must either fit within the existing structures, or the existing structures must be revised to make accommodation possible (in the case of transformative change). For this reason, Connelly and Clandinin (1994 ) use the term knowledge reconstruction. They argue that reconstruction of narrative knowledge, by retelling teaching/learning stories, can lead to "awakenings and transformations" that promote such change (p.158).

According to Handal (2004), teachers' beliefs are a central issue in the study of teacher change. This is because, where the goal of professional development is transformative change, research shows that new practices need to be underpinned by compatible beliefs if they are to become embedded into day-to-day practice in a sustainable way (Handal, 2004; Handal \& Herrington, 2003; Keys, 2006; Martin, 1993; Smith et al., 2005). Incompatible beliefs and frameworks need to be replaced or restructured to better fit the emerging paradigm. Some theorists see beliefs as an inseparable part of teachers' knowledge (e.g. (Bromme \& Tillema, 1995).

The literature reviewed above provides no ready answer to the question of how new knowledge becomes embedded by established professionals, but it emphasises the 
significant challenges that are involved once personal knowledge constructs have become established. This is a key issue in the context of challenging, transformative change. I will now consider research that studies the embedding of knowledge at system or macro level.

\subsubsection{Embedding knowledge in systems (a macro-level view)}

As my research context is one where system-level KT and embedding is involved, and as online CoPs typically span organisational boundaries, it is relevant to consider research that concerns the embedding of knowledge at a system, or macro, level. Research at this level concerns processes across a range of levels, such as individual, organisation, community, network, and system and their interrelationships. Systemlevel KT studies typically apply one of several types of theory: DOI Diffusion of Innovations (DOI) theory $(1962,1983,2003)$; a hybrid of DOI and KM theory, Theory of Action, and Ecological Theory. I consider each of these and summarise issues of relevance to my study.

\subsection{DOI and Knowledge Diffusion \& Utilisation (KDU) theory} Rogers' DOI theory (ibid) was designed to explain the progressive uptake of an innovation in society. It has been applied, with limited success, to explain KT or knowledge diffusion and utilisation (KDU) and institutionalisation in health and education (Greenhalgh et al., 2005; Hutchinson \& Huberman, 1993). Recent studies in this tradition use the term knowledge mobilisation (e.g., Cooper et al., 2009).

KDU research studies the dissemination of research-based knowledge and its uptake and institutionalisation. (A KDU lens appears to frame the NZ government's discourse about using knowledge to transform education.) This research is characterised by a top-down view of KT and a change orientation: the knowledge at stake is designed to replace existing knowledge. KDU research is typically based around a simplistic threestep model of knowledge transfer: creation, dissemination and utilisation. The third stage is where one might expect embedding to occur.

KDU research is, however, distinguished by poor theoretical development (Rich, 1991) arising from the use of problematic frameworks and the difficulty of integrating literature from multiple disciplines. In a 1991 literature review, Friedman and Farag (1991) describe their inability to synthesise a literature in excess of 10,000 studies, spanning over 18 disciplines, and using diverse terminology. In addition, in a review of KDU research in educational change, Hutchinson and Huberman (1993) found that linear 
diffusion models are unsuccessful in rooting new knowledge in place because they do not account for the motivations, contexts, and realities of the intended recipients (ibid). In the field of heath, Farkas et al (2003) note that, "the need to institutionalize knowledge in daily practice has long been recognised as one of the most difficult aspects of knowledge utilization" (ibid, .50). Rich (ibid) has highlighted a combination of serious conceptual gaps, unanswered questions, conflicting findings and poorly developed theory in KDU research (pp 334-335). These problems have persisted. In a review of research into the diffusion and utilisation of service innovations Greenhalgh et al. (2004) note a gap in the understanding of:

...the detailed process by which ideas are captured from outside, circulated internally, adapted, reframed, implemented, and routinized ....and how...this process [might] be systematically enhanced. (p. 618)

These findings are unsurprising, given the lack of attention in this research tradition to embedding - a process which $\mathrm{KM}$ research shows to be vital and complex. As Greenhalgh et al. (2005) note, the problem is that DOI theory, in which an innovation is seen as present or absent, is too simplistic to be applied to KT studies.

\subsection{Hybrid KDU and KM theory}

In light of the problems with DOI-based theory (noted above), some researchers have developed hybrid models. Farkas et al's (2003) 4E's framework (used by Boston University's Roybal Centre) blends KM concepts with DOI theory. It takes into account the need for attitudinal and behavioural change in the case of a paradigm shift and comprises strategies for exposure, experience, expertise and embedding with goals aligned to different target populations. The embedding strategies for providers are organisational change (developing structures to support the use of new knowledge), power strategies (establishing new rules), and resource-based strategies (changing funding models). Support in developing experience and providing expertise is also needed. For health consumers, embedding is seen as requiring personally focused intervention, peer support and feedback tools. This framework does not explain how professional knowledge is embedded, but it recognises complexity, giving an overview of the kind of strategies needed when the knowledge to be embedded is transformative. It also provides a continual process view of the embedding process. (The duality observed between the linear and continual process views of embedding in the organisational literature is replicated at this macro level.) A weakness of the model is that it fails to consider the needs of individuals. The findings of Nonaka and Orlikowski concerning the social dimension of knowledge, combined with research 
findings about the difficulty of individual professional change, suggests a need for guidance in this area.

\subsection{Accounting for knowledge construction at a system level}

Research in the institutionalisation of educational research has recently taken a constructivist perspective, viewing the teacher as actively engaged in the creation of their own knowledge rather than as a passive receiver (Hutchinson \& Huberman, 1993). Problem-solving (ibid) and dialogic engagement (McGregor et al., 2006) are seen as key facilitators of knowledge utilisation. This research positions embedding as being based around social interactions. Key requirements are two-way communications and iteration. Teachers learn new practices and new ways of thinking by constantly readjusting their understandings and checking with experts and intermediaries as new practices are introduced (Hutchinson \& Huberman, 1993). Constructivist theory is incompatible with the problematic dissemination-based model, so alternative ways of viewing $\mathrm{KT}$ at a system level must be considered. I now briefly review the application of Ecological Theory and Theory of Action to KT research.

\subsection{Ecological theory}

In the social sciences, many studies of system-level change have been conducted using ecological, or bio-ecological systems (BS) theory (Bronfenbrenner, 1979, 2001, 2005b). This theory holds that decisions and behaviours are mediated by one's social and physical environment through a complex system of reciprocal behaviourenvironment interactions. We sit in the centre of an ecology made up of a series of overlapping ecosystems - the microsystem(s), mesosystem, exosystem and macrosystem - "a set of nested structures, each inside the next, like a set of Russian dolls" (Bronfenbrenner, 1979, p.22). The individual is impacted on by this ecology and in turn influences it, creating a two-way cycle that is strongly sensitive to change. There are interactions between systems at all levels (Bronfenbrenner, 2005a; Lerner, 2002, 2005). Within each system, roles, norms and rules help shape human behaviour and development. The influence of the system is in turn moderated by our perception.

BS theory has been used as a tool for planning system-level interventions and as a research framework for investigating programmes focused at a societal level, such as studies of the implementation and institutionalisation of health promotion programmes (Reynolds et al., 1997) and of educational reform. Lewthwaite (2006) used it to identify multi-system factors contributing to teachers' delivery of science programmes, highlighting the roles involved in affecting change. McLeroy et al. (1988) developed a 
modified ecological model identifying five leverage points for embedding of knowledge in the context of health promotion programmes: intrapersonal factors, interpersonal processes, institutional factors, community factors and public policy.

BS research is strongly contextual, so the main source of relevance of these studies to my research lies not in the findings of specific studies but in the BS framework itself. It can be seen as potentially suitable for accounting for the factors impacting on knowledge embedding at the individual, school, community and system levels, and for the interactions of these. However, there are two drawbacks: Firstly, the model is essentially factor-focused. While it could be used to identify key moderators of embedding and their interactions, it is unsuitable for accounting for a complex process that occurs over time. Secondly, BS theory is firmly focused on the individual and sees larger change as the cumulative result of change in many individuals. The embedding targets in my research context were both schools and individuals. KM research makes it clear that the embedding of knowledge in organisations is more than the sum of embedded knowledge in individuals. (Davenport (1997) has adapted Bronfenbrenner's idea of nested systems to create a model for an information ecology. The organisation, rather than the individual is placed at the centre. This creates the converse situation: a lack of emphasis on the individual.

\subsection{Theory of Action}

A series of studies commissioned by UK's National College for School Leadership (NCSL) investigated the nature of networked learning communities and their impact on schools, based on the 2002-2006 Networked Learning Communities (NLC) programme. This programme studied 134 school networks, involving around 35,000 staff and over 675,000 pupils. It was an exercise in studying lateral KT from a constructivist lens. The programme has produced a 36-page directory of publications ranging from theoretical syntheses to evaluative studies (Bell et al., 2006; Earl et al., 2006; Goodfellow, 2003; McCormick, 2002; McGregor et al., 2006; Thorpe, 2003a, 2003b; Thorpe \& Jelfs, 2004). These studies do not explain how knowledge is embedded, but they provide useful information about the facilitators of embedding at multiple levels.

The NCL programme design used a levels of learning framework to investigate how KT and professional dialogue were managed at six levels - pupil, adult, leadership, school, school-to-school and network-to-network (Bell et al., 2006). The final report (Earl et al., 2006) uses a Theory of Action (TOA) framework to explain the reciprocal influence of 
schools and networks on each other, leading to professional creation and sharing. (The theme of embedding is not addressed. It is also worth noting that fewer than half of schools involved reported having experienced "changes in thinking and practice", p.21 and that online interactions played an insignificant role.)

TOA was developed by Parsons $(1937,1949,1951)$ and modified continuously throughout his life (Fox et al., 2005). It explains dynamic processes in terms of a social system and the relationships of its parts. It is beyond the scope of this review to analyse its development and applications, but at its simplest, it sees a social action as based on changes in four elements of social structure: ends, means, norms and conditions. Earl et al.'s (2006) model is loosely based on this approach. While it does not explain how embedding occurs, it is useful in providing a broad-brush view of factors that were associated with professional knowledge creation and sharing present in the networked communities.

At the network level, the key factors contributing to professional knowledge creation and sharing were: focus, formal leadership, distributed leadership, enquiry culture, relationships, collaboration and joint work that challenges thinking and practices. The same factors operate at school level, along with developing capacity for collaborative enquiry. These two sets of factors have a reciprocal influence on each other. The school level conditions exert the main influence on professional knowledge creation. This in turn leads to distributed, deep and sustained changes in practices and structures in schools, which impact on student learning, engagement and success in a knowledge society (Earl et al., ibid p.6). The authors note that a drawback of their theory is that "it is difficult to disentangle the key features to isolate ones that are essential from the others" (p.77).

The TOA model (ibid) stops well short of explaining the embedding process, but it is useful in highlighting (a) the preconditions of knowledge creation in a network-ofcommunities based system, and (b) the complex structures and interactions that are involved when studying KT at a systems level. (DOI theory does not adequately account for this.) Earl et al.'s study raises a third issue of significance to my study; the problem of the invisibility of network level influences in a system. The authors note that:

The work of networks of schools is almost always indirect, evidenced in changes that occur in schools and classrooms removed from the network by time and space. This makes it very hard to establish any direct links. Even the people who are most knowledgeable about the networks don't always see their influence...At the same time, there is 
evidence from this study that attachment to the network and widespread involvement in it are related to changes in thinking and practice and to pupil learning. (p.14)

There is a paradox in that what may be a key source of value can be invisible to the players involved. This is of concern in a global environment where whole-of-system reform strategies are coming of age (Fullan, 2009) yet governments require robust evidence of the outcomes of system-level interventions.

A TOA framework is more suitable than the models used in much KDU research for accounting for the reciprocal levels based at different system levels, but it does not account for how $\mathrm{KT}$ or embedding occurs.

\subsection{The nature of macro-level theory}

In this section, I have not aimed to summarise all possible macro-level theories that could be used to account for KT and embedding, but to draw attention to the drawbacks of some existing macro-level models and highlight issues to do with macrolevel theory generally. The fundamental problem is that the nature of macro level theory means it must focus on system level relationships at the expense of process. This applies to theoretical frameworks other than the ones discussed in depth. For example, McCormick (2002) notes the limitations in using Engeström's Activity Theory ${ }^{3}$ to account for the learning processes that occur in networked learning communities:

It is not clear how it helps either with the analysis of the situation or any prescriptions that might be useful to those involved in the networks. (p.14)

This raises a methodological issue for my study: Because my research is concerned with elucidating the nature of the embedding process as well as the facilitating factors, a macro-level framework can be seen as unsuitable.

\footnotetext{
${ }^{3}$ Activity theory focuses on "the learning and development that emerge in the institutionalized contexts of culturally and historically mediated social practical activities" (Yamazumi, et al., 2007, p.44).
} 


\subsubsection{Section summary: Embedding professional knowledge}

In this section I have synthesised literature from diverse fields to ascertain what is known about embedded knowledge, professional knowledge and the knowledge embedding process. Owing to considerable diversity in how embedded knowledge is described in the literature, I have synthesised a working definition. I have argued that, although embedded knowledge may have a strong tacit dimension, it is not synonymous with tacit knowledge. It is characterised primarily by its highly contextual nature - the extent to which it has been personalised or customised and integrated with other knowledge. Professional knowledge exists at organisational and individual levels. While in constant interaction, these kinds of knowledge are very different. Individual professional knowledge is a complex, personal construct.

This section has also justified knowledge embedding as a relevant research topic. Embedding of knowledge is needed to foster organisational currency, effectiveness, flexibility and alignment; yet when it comes to change, a key barrier to embedding new knowledge is the knowledge that is already embedded. Individual and organisational beliefs and interpretive frameworks must be consonant with new knowledge in order for sustained embedding to occur. When transformational knowledge needs to be embedded, beliefs, values and interpretive frameworks should therefore be seen as part of the package. This underlines the importance of my research question. Research that specifically considers knowledge embedding - as distinct from the overarching KT process - is scarce. This area has been identified as a significant gap in the KM field and a hot topic for research (Argote et al., 2003).

My analysis of KM literature has uncovered a dual perspective on how the embedding process may occur. Some researchers emphasise linear process models and the importance of temporal moderators of embedding, but others focus instead on portraying embedding as a continual process that drives personal and organisational knowing or knowledge creation. The duality occurs in both organisational and systemlevel research. I have argued that the two perspectives are equally important and that there is a need for an integrated approach.

My research setting is one of systemic change and CoPs typically span organisational boundaries. I have therefore reviewed literature operating at three levels, providing a three-tier view of how knowledge embedding has been addressed: Tier 1 is a mesoleve/ view based on an organisational perspective; tier 2 is a micro-level view focusing 
on the individual professional; and tier 3 is a macro-leve/ view that focuses on the system and the relationships between components of the system. These views are not nested within each other. Rather, they provide complementary perspectives, depending on the unit of concern and the closeness of scrutiny involved.

As one zooms in from a birds-eye view, a portion of the landscape becomes clearer, at the expense of the field of vision. As one zooms out again the detail is lost, for the sake of the big picture. Macro-level models are of value in capturing the different kinds of activities and interventions needed at each level as well as the relationships between these. They therefore cannot offer a sufficiently granular view to explain how embedding operates at the meso or micro levels. Likewise, as the literature review has shown, the organisational (meso) level perspective of embedding does not explain how knowledge is embedded at the individual (micro) level, and vice versa. A multi-level, or three-tier, view is of value in that it provides the level of detail and degree of focus that is most appropriate to each level. (This view, along with its limitations, is mirrored in the way I have presented my research results. I did not, however, set out to produce multilevel theory; as I explain later in this thesis.)

In the next section of this literature review, I focus on the research into online CoPs and how they might facilitate the embedding of professional knowledge. 


\subsection{Online CoPs and knowledge embedding}

\subsubsection{Introduction}

In this section I review literature relating to online CoPs. Although the literature suggests that online CoPs can provide a supportive environment for $K T$, and pays considerable attention to moderators of knowledge sharing behaviour, there is no research that explains how they might facilitate the actual KT and embedding process. I therefore provide an overview of the CoP literature, highlighting themes and studies that are of potential relevance.

\subsubsection{Traditional CoPs}

The earliest CoP research, conducted in offline settings, concerned the transfer and embedding of professional knowledge. Lave and Wenger, in their seminal (1991) study of apprenticeship found that novices acquired knowledge and skills from experts in the context of everyday activities. According to their Situated Learning theory, participation in social workplace settings is inseparable from learning. The ongoing interactions between an individual and their social context determine the understandings they develop (MaKinster et al., 2006). This is similar to Nonaka's concept of socialisation (1995) and Orlikowski's knowing (2002).

A CoP is a group of people who interact regularly and are united by their shared interest or profession, and the value they place on shared learning in that area (Wenger, 1998b). CoPs are distinguished by: (1) a sense of joint enterprise around a topic of interest, creating shared accountability to a body of knowledge, (2) relationships of mutual engagement and (3) a shared repertoire of resources such as artefacts, assumptions, language and understandings (ibid). These features facilitate innovation, knowledge exchange and learning (ibid) and help workers explore the meaning of their practice and develop a professional identity (Gray, 2004; Hew \& Hara, 2007a). CoPs may cross the boundaries of institutional hierarchies, cultures, countries, and organisations (ibid), providing a common focal point.

CoPs also provide value to organisations. They can facilitate organisational learning by supporting the sharing of distributed knowledge as it is created, valued and understood (Brown \& Duguid, 1991) and may help improve organisational performance by fostering three types of social capital: structural (connections amongst workers), relational (trust and mutual obligation), and cognitive (shared language and context) 
(Lesser \& Storck, 2001, p.831; Teigland \& Wakso, 2004). As a result, CoPs have been utilised by organisations for "what amounts to an interventionist strategy...seeking to harness the learning potential of workers for the benefit of the organisation" (Thorpe \& Jelfs, 2004).

\subsubsection{Online CoPs}

In the late twentieth century, with the arrival of internet-mediated communications and a growing awareness of the value of knowledge, CoPs gained a new online dimension as organisations began to utilise online CoPs to support KM initiatives. Online CoPs are CoPs whose members rely on ICT and the Internet to communicate, although they may also meet face-to-face (Dubé et al., 2006; Lai et al., 2005). Online CoPs may therefore combine the use of traditional media (e.g., phone, teleconference, fax) with the use of online tools (e.g., e-mail, forum, chat, on-line meeting space, videoconference, newsgroup, shared database, website, and intranet) to establish a shared virtual collaborative space (Lai et al. ibid, p.70).

The terminology used in studies of online CoPs is far from consistent: Wenger et al. (2002) use the term distributed CoPs, while Hew and Hara (2007) use the term computer-mediated CoPs. Hemmasi and Csanda (2009) dispense with a prefix altogether, seeing online communication as integral to modern CoPs. The term virtual $\mathrm{COP}$ is used to refer to a subset of online CoPs whose members rarely, or never, meet face-to-face (e.g.,Dubé et al., 2006; Saint-Onge \& Wallace, 2003). Brown and Duguid (2000) and Wasko and Teigland (2004) use the term electronic networks of practice (NoPs) for such groups, arguing that CoP members must be co-located in order to have strong ties. This view is challenged by Murillo (2008) who has identified online CoPs that exhibit Wenger's CoP constructs within Usenet discussion networks. I have used the term online CoPs owing to its inclusiveness.

\subsubsection{The value of online CoPs for supporting $K T$}

Since the late twentieth century, the growing appreciation of the importance of KM by organisations has resulted in the development of various "KM" technologies. Davidson and Voss (2002) identify two broad approaches: codification-based systems, based around capturing and codifying knowledge; and personalisation-based systems that aim to support knowledge sharing amongst people. Online CoPs are seen as having potential to integrate these approaches, providing members with access to a repository of collective contributions while facilitating direct interaction (Zhang \& Watts, 2003). However, there seems to be an increasing emphasis on using CoPs to support the 
personalisation approach: Wagner and Bolloju (2005) have noted that inexpensive technologies for supporting human interaction - discussion forums, weblogs (blogs) and wikis, or conversational technologies - are now the most popular KM technologies and that these tools are ideal for supporting CoP needs such as daily question answering, access to a diverse group of experts and incremental knowledge refinement (p.vii). At a higher level, this trend may reflect a reported shift in KM practice away from a computer-centred focus towards one that is more human-centred (Lee \& Lan, 2007; Schmitz-Justen \& Wilhelm, 2005).

Various claims have been made about the role of online CoPs in facilitating KT amongst professionals. They are seen as militating against the barriers of distance, time and professional isolation, increasing opportunities for knowledge sharing, making interactions more visible, sustaining these interactions and extending their reach (Davenport, 2004; Hara \& Kling, 2002). Johnson (2001) suggests that in an online CoP setting where collaboration is text-based, norms change, making it easier for introverts to share ideas with extraverts (p.45). Studies of online learning communities suggest text-based environments may act as an equaliser, transcending social or physical barriers to participation (Baker-Eveleth et al., 2005; Harasim, 1990). Through text-based dialogue, participants can reduce psychological distance (Rovai, 2002), developing shared understanding through "a volleying of ideas, meanings and understandings" (Baker-Eveleth et al.,p.5). Wagner and Bolloju (2005) draw on constructivist learning theory, suggesting that "the process of expressing knowledge [in an online $\mathrm{CoP}$ ] helps people to construct it (cognitive constructivism) as the conversation helps in refining the knowledge (social constructivism)" (p. ii).

Online CoPs are considered to be useful for supporting professionals who need to keep up with a changing knowledge base (Hargreaves, 2003; Hew \& Hara, 2007b). They may even have a transformative potential: The collective building of an archive of knowledge is seen by Hara and Kling (2002) as promoting the kind of reflective practice which can lead to learning in the form of modified understandings and actions (Dewey, 1933; Schön, 1983).

There is relatively little research that provides robust evidence to support these purported benefits of online CoPs. Although it is known that KT can occur in distributed online settings (e.g., Sarker, et al., 2005; Zhang \& Watts, 2003), there is a poor understanding of how this occurs; particularly of how embedding might occur. There is, however, a set of studies that examine the moderators impacting on KT in online CoPs. 


\subsubsection{Moderators of KT in online CoPs}

Research into KT in online CoPs is typically underpinned (explicitly or implicitly) by an input-output, or transmission-based, model in which KT is seen as arising from the giving of knowledge by a source and its receiving by a recipient (e.g.,Schmitz-Justen \& Wilhelm, 2005; Sharatt \& Usoro, 2003; Zhang \& Watts, 2003). Studies in this tradition are concerned with identifying factors that encourage and/or inhibit users' participation (e.g., Ardichvili et al., 2003; Yoo et al., 2002), or knowledge sharing (e.g., Hew \& Hara, 2007a; Schmitz-Justen \& Wilhelm, 2005; Sharatt \& Usoro, 2003; Wasko \& Faraj, 2005). For example, based on a literature review, Sharrat and Usoro (2003) propose a framework of five factors influencing the decision to share knowledge in online CoPs: knowledge sharing (contribution), organisational structure, information system, trust and recognition. Trust is known to be a critical facilitator of $\mathrm{KT}$, encouraging risk taking and new ways of negotiating meaning members (Brosnan \& Burgess, 2003; Lai et al., 2005). Closely related is the need to feel valued. McDermott (2000) found that while members join a CoP because of interest, they stay involved because of their sense of belonging. The perceived credibility of others is another moderator: In an exploratory study of virtual teams, Sarker et al. (2005) identified three characteristics needed for people to be seen as effective KT agents: extensive online communication, perceived credibility and collectivist values. (There may be a stronger imperative for KT to occur in virtual teams than in CoPs, but these findings nonetheless seem relevant.)

In a more recent literature review, Hew and Hara (2007b) identify six categories of motivators for sharing knowledge in online environments: reciprocity, personal gain, altruism, collectivism, ease of technology use and external goals. Using multi-case analysis they found that the most common motivator was a combination of collectivism and reciprocity. An interesting outcome of this study was the finding that different combinations of motivators and barriers exist in individuals, and that these may impact in an integrated way on knowledge sharing (ibid, p.2322). Barriers appear to vary according to context, but may include lack of time, unfamiliarity with the subject, having nothing to add, technology issues, confidentiality concerns, the perceived inability to use knowledge and fight avoidance.

The studies considered are valuable in that they recognise the critical role of the individual in engaging, articulating and interpreting knowledge in online settings. However, they have a number of limitations. Firstly, the lack of consistency suggests that the motivations for sharing knowledge may be contextual and even practice dependent: Hew and Hara (2007) note that while collectivism and reciprocity impacted 
positively on knowledge sharing amongst teachers, this did not apply in a study of lawyers (Wasko \& Faraj, 2005). Secondly, an input-output model of KT is problematic: Unless KT is conceived of as a process that may be both complex and gradual - as the $\mathrm{KM}$ literature suggests it is - the issue of how KT occurs does not arise. The emphasis in studies may easily rest on finding the enablers of more superficial sharing behaviours, such as the one-off use of an idea.

Thirdly, the emphasis on user-related moderators of KT can be seen as disproportionate in relationship to the full range of factors known to be involved: Based on Szulanski, Sarker et al. (2005) propose a framework of five elements that may influence KT: channel, message, context, recipient and source (p.203). Although developed for use in studying virtual teams, this model could be usefully applied to enrich research into KT in online CoPs. There is a relative paucity of research into the impact of channel, message and context. Notably, while studies of traditional CoPs emphasise the crucial importance of context in KT (Brown \& Duguid, 1991; Brown \& Duguid, 2000; Lave \& Wenger, 1991), concern with context is an emergent area in the online CoP literature. It is an area that appears to offer potential: In a study of an online student teacher community MaKinster et al. (2006) found that different online contexts impacted on the nature of members' reflections and their perceptions of value.

Reflection amongst colleagues in a forum was a more social practice than reflection in a private journal and was more valued. Reflection amongst experienced teachers was valued even more. These findings suggest that social context may exist online and that it may indirectly influence $\mathrm{KT}$.

Zhang and Watts' (2003) explored how contextual message-related factors may impact on knowledge adoption in online CoPs, applying dual process theory. This theory holds that there is a direct (central) and an indirect (peripheral) route to one's understanding. The central route is based on information content and involves cognitive, systematic thinking. The peripheral route is based, on the other hand, on information context. It involves validity assessments based on heuristic cues. The two modes co-exist, having variable levels of influence (Gilbert, 1999; Zhang \& Watts, 2003). The authors used a survey to explore the interaction and impact of various factors on the two modes. Their results suggest that genre conformity (conformity to the norms of use for a given message genre, such as a forum) and information consistency may operate as heuristic cues in online CoPs. The impact of these cues decreased as users became more involved. Focused search and disconfirming information increased recipients' use of the direct route. With increasing levels of user expertise, the impact of argument 
quality increased. Also, as users undertook more focused search, the impact of source credibility decreased. This study, while exploratory and perhaps overly complex for a survey, highlights the likely complexity and interconnectedness of contextual factors that may affect $\mathrm{KT}$, and their interplay with user behaviour.

This section has summarised research concerning the moderators of KT in online CoPs. In the previous section, a review of the KM literature has exposed a duality of perspectives on how KT occurs - there is a continual process view and a stages-ofchange view. (I have suggested that these are complementary views and that it is useful to understand both.) The CoP literature provides no such duality. When concerned with identifying moderators of $\mathrm{KT}$, the CoP research takes an ongoing process view of KT, often based on an input-output model. This literature does not consider the possible stages of KT that may occur in conjunction with change and therefore does not examine the possible stage-dependency of KT moderators. Although claims have been made that online CoPs might support professional change (e.g., Hargreaves, 2003) current research does not provide a good basis for understanding how this might occur. This is an important area for future consideration.

\subsubsection{Developmental stages of CoPs}

The theme of developmental stages or a lifecycle of the CoP itself has, however, received attention from a group of authors (Allee, 2000; Gongla \& Rizzuto, 2001; Lai et al., 2005; McDermott, 2000; Wenger, 1998a; Wenger et al., 2002). The common theme is that, according to their stage of development, CoPs exhibit different characteristics, face different challenges, require different roles, undertake different activities, and differ in the nature of value that they provide (ibid). This literature is of relevance for methodological reasons: Depending on one's research question, when selecting CoPs for inclusion in a study it may be necessary to take this into account.

Table 4 compares six developmental models. Two are lifecycle models (McDermott, 2000; Wenger, 1998a), two employ an evolutionary capability development perspective (Gongla \& Rizzuto, 2001; Wenger et al., 2002), and two are syntheses (Lai et al., 2005; Tarmizi \& De Vreede, 2005). Lifecycle models view development as a one-way process, whereas evolutionary capability models allow for maturing or dissolving at any stage. Rather than recount the models in detail or debate their relative merits, I consider when, in relationship to a CoP's development, KT and embedding might be expected to occur. 


\subsection{Early stages}

In the early stages of development, CoP structures, systems and relationships are established. Key activities are building membership and connections, finding common ground, building a common vocabulary, designing the community, formalising operating principles, building trust, establishing core members, and generating energy. These stages can be seen as setting the foundational conditions for subsequent KT and embedding.

\subsection{Middle stages}

In the middle stages, the CoP is mature and its members actively share knowledge. It seems that the conditions are now right for KT and embedding to occur. In Gongla and Rizzuto's Engaged stage the focus is on access and learning. Enabling people behaviour includes developing trust, loyalty and commitment, modelling knowledgesharing, telling community stories and building the knowledge base. Process support includes supporting tacit knowledge exchange, developing and disseminating communications, gathering and managing feedback, correcting problems and adjusting. In the Active stage, the focus is collaboration (ibid).

Key issues in the Maturing stage include managing boundaries to avoid distraction, and shifting from sharing to organising knowledge (Wenger et al., 2002). The CoP may begin to share an explicit learning agenda. At the Stewardship stage, key concerns include maintaining relevance, finding a voice in the organisation, keeping the tone and intellectual focus lively and challenging, and staying at the cutting edge (Wenger, et al., 2002). CoPs may find their domain, membership and practice expanding. To stay relevant, they may need new ideas and approaches. Stewardship requires managing tensions between ownership and openness, and between maintaining focus and expanding. These tensions may pose a challenge to the embedding of knowledge.

\subsection{Late stages}

In the final stages, CoPs close, adapt or transform. Members may disengage and activities may cease. According to Wenger's (1998a) model, the CoP is inactive in the final Memorable stage, but it retains its memories, stories and artefacts. (This may facilitate reflective practice, promoting embedding at an individual level.) While Wenger et al.'s later (2002) model dispenses with this stage, it is used by Tarmizi and de Vreede (2005). Unfortunately there is a lack of research into CoPs at this late stage of development. 


\begin{tabular}{|c|c|c|c|c|c|}
\hline \multicolumn{2}{|c|}{ Lifecycle models } & \multicolumn{2}{|c|}{$\begin{array}{l}\text { Evolutionary capabllity } \\
\text { models }\end{array}$} & \multicolumn{2}{|c|}{ Syntheses } \\
\hline Wenger (1998B) & MeDermott (2000) & Gongla \& Rizzuto (2001) & $\begin{array}{l}\text { Wenger, McDermott \& } \\
\text { Snyder (2002) }\end{array}$ & $\begin{array}{l}\text { Tarmizl \& de } \\
\text { Vreede (2005) }\end{array}$ & Lad et al (2005) \\
\hline 1. Potential & 1. Planning & 1. Potential & 1. Potential & 1. Potential & 1.Formation \\
\hline $\begin{array}{l}\text { Pcople face similar } \\
\text { situations without the } \\
\text { benefit of a shared } \\
\text { practice. Finding each } \\
\text { other, exploring } \\
\text { commonalities }\end{array}$ & $\begin{array}{l}\text { A network with a topic of } \\
\text { interest and the potential } \\
\text { for growth and value is } \\
\text { discovered. Planning } \\
\text { commences }\end{array}$ & $\begin{array}{l}\text { A community is forming } \\
\text { Fundamental function: } \\
\text { conncction }\end{array}$ & $\begin{array}{l}\text { CoP starts as loose } \\
\text { network that has } \\
\text { potential to become } \\
\text { more conneeted \& create } \\
\text { valuc } \\
\text { Key issue: Finding } \\
\text { common ground }\end{array}$ & $\begin{array}{l}\text { Potential members } \\
\text { explore the } \\
\text { possibility and } \\
\text { desirability of } \\
\text { sctup }\end{array}$ & \multirow[t]{3}{*}{$\begin{array}{l}\text { Identify potential } \\
\text { community; determine } \\
\text { purposc and scope; build } \\
\text { community; create } \\
\text { preliminary design for } \\
\text { community; Incubate and } \\
\text { deliver immediate value; } \\
\text { and launch of community. }\end{array}$} \\
\hline 2. Coslescing & 2. Start-up & 2. Building & 2. Coalescing & 2. Buildiug & \\
\hline $\begin{array}{l}\text { Mcmbers come } \\
\text { together and recognise } \\
\text { their potential } \\
\text { Exploring } \\
\text { connectedness, } \\
\text { defining joint } \\
\text { enterprise, negotiating } \\
\text { community }\end{array}$ & $\begin{array}{l}\text { The CoP starts (typically) } \\
\text { with a spark of interest, } \\
\text { then energy falls. Trust } \\
\text { and value begin to be } \\
\text { built. A 'core' group of } \\
\text { regular members inspires } \\
\text { others. }\end{array}$ & $\begin{array}{l}\text { The eommunity defines } \\
\text { itself and formalises its } \\
\text { operating principles } \\
\text { Fundamental function: } \\
\text { memory and context } \\
\text { creation }\end{array}$ & $\begin{array}{l}\text { Members build } \\
\text { connections, and } \\
\text { coalesce into a CoP } \\
\text { Key issue: gencrating } \\
\text { energy to coalesce, } \\
\text { demonstrating valuc }\end{array}$ & $\begin{array}{l}\text { The CoP is set up } \\
\text { but has not } \\
\text { officially started } \\
\text { (it may run as a } \\
\text { pilot) }\end{array}$ & \\
\hline 3. Active & 3. Growth & 3. Engaged & 3. Maturing & 3. Active & 2. Sustaining/Maturing \\
\hline $\begin{array}{l}\text { Members engage in } \\
\text { developing the } \\
\text { practice. Joint } \\
\text { activities, creation of } \\
\text { artifacts, adapting, } \\
\text { renewing interest. } \\
\text { commitment\& } \\
\text { relationships }\end{array}$ & $\begin{array}{l}\text { Growth brings new ideas } \\
\text { and members and } \\
\text { increases exposure. } \\
\text { Vibrancy increases and } \\
\text { patterns are disrupted. } \\
\text { Tension between growth } \\
\text { and focus needs to be } \\
\text { managed }\end{array}$ & $\begin{array}{l}\text { The community executes } \\
\text { and improves its } \\
\text { processes } \\
\text { Fundamental function: } \\
\text { access and learning }\end{array}$ & $\begin{array}{l}\text { The CoP grows in terms } \\
\text { of membership and often } \\
\text { also the depth of } \\
\text { knowledge that is shared } \\
\text { Key issue: Clarifying } \\
\text { focus, role and } \\
\text { boundaries }\end{array}$ & \multirow{3}{*}{$\begin{array}{l}\text { The CoP has } \\
\text { officially started, } \\
\text { it takes off, it } \\
\text { shows rapid } \\
\text { growth, and } \\
\text { becoumes a routine } \\
\text { part of the } \\
\text { organisation (this } \\
\text { stage combines } \\
\text { Gongla \& } \\
\text { Rizzuto's stages } \\
3,4 \& 5 \text {; or } \\
\text { Wenger's stages } \\
3 \& 4 \text { ) }\end{array}$} & \multirow{3}{*}{$\begin{array}{l}\text { Focus is on sustaining and } \\
\text { maturing the CoP through } \\
\text { leadership, mentoring new } \\
\text { members, seeking } \\
\text { relationships \& henchmarks } \\
\text { outside the organisation; } \\
\text { establishing the } \\
\text { community; undertaking a } \\
\text { "checkpoint"; building a } \\
\text { knowledge repository; and } \\
\text { evaluating purpose \& } \\
\text { direction. }\end{array}$} \\
\hline 4. Dispersed & 4. Sustain & 4. Active & 4. Stewardship & & \\
\hline $\begin{array}{l}\text { Members no longer } \\
\text { engage intensely, but } \\
\text { the CoP still functions } \\
\text { as a force and centre } \\
\text { of knowledge. Staying } \\
\text { in touch, } \\
\text { communicating, } \\
\text { calling for advice, } \\
\text { reunions } \\
\end{array}$ & $\begin{array}{l}\text { The mature community } \\
\text { maintains momentum } \\
\text { during changes in } \\
\text { membership, technology, } \\
\text { environment. Leaders } \\
\text { bring new members and } \\
\text { present new challenges. } \\
\text { Sense of ownership and } \\
\text { intimacy is high } \\
\end{array}$ & $\begin{array}{l}\text { The community } \\
\text { understands and } \\
\text { demonstrates benefits } \\
\text { from KM and the } \\
\text { collective work of the } \\
\text { community } \\
\text { Fundamental function: } \\
\text { collaboration }\end{array}$ & $\begin{array}{l}\text { CoP goes through cycles } \\
\text { of high \& low activity. It } \\
\text { may take more active } \\
\text { stewardship of the } \\
\text { knowledge and practices } \\
\text { they share, consciously } \\
\text { developing them. Key } \\
\text { issue: sustaining } \\
\text { momentum } \\
\end{array}$ & & \\
\hline 5. Memorable & 5. Close & 5. Adaptive & 5 Transformation & 4. Inactive & 3. Transformation \\
\hline $\begin{array}{l}\text { The CoP is no longer } \\
\text { central, but people still } \\
\text { remember it as a } \\
\text { significant part of their } \\
\text { identities. Telling } \\
\text { stories, preserving } \\
\text { astifacts, collecting } \\
\text { memorabilia }\end{array}$ & $\begin{array}{l}\text { The community fades } \\
\text { away, turns into a 'social } \\
\text { club', or is closed }\end{array}$ & $\begin{array}{l}\text { The community and its } \\
\text { supporting organization(s) } \\
\text { are using knowledge for } \\
\text { competitive advantage } \\
\text { Fundamental function: } \\
\text { innovation and generation }\end{array}$ & $\begin{array}{l}\text { An event, new members, } \\
\text { or a drop in energy leads } \\
\text { to a transformation, a } \\
\text { retum to an earlier stage, } \\
\text { or closure. Key issue: } \\
\text { Not stated }\end{array}$ & $\begin{array}{l}\text { Members are no } \\
\text { longer active and } \\
\text { do not use the } \\
\text { community, but } \\
\text { people remember } \\
\text { it as part of their } \\
\text { identities }\end{array}$ & $\begin{array}{l}\text { Transformation or } \\
\text { disengaging. CoPs may } \\
\text { face expansion, fading } \\
\text { away, and/or death }\end{array}$ \\
\hline
\end{tabular}

Table 4: Stages of CoP development: Comparison of six models, highlighting when knowledge embedding is likely to occur.

\subsection{Summary: Developmental stages of CoPs}

A review of literature on the stages of CoP development suggests that embedding is most likely to occur at the middle and/or late stages of a CoPs lifecycle. As a CoP matures, its focus moves on to learning, knowledge building and systematising knowledge. This can be seen as requiring a significant amount of KT. As it enters the late stages, the repository may facilitate reflective practice, leading to embedding of knowledge. The structures that facilitate the embedding of knowledge appear to be laid in place much earlier. The implication is that, unless conducting a longitudinal study, it would be most useful to select CoPs that are well established if studying the phenomenon of embedding. 


\subsubsection{Roles in online CoPs}

One of my research sub-questions concerns how roles contribute to the embedding of knowledge through online CoPs. I therefore briefly summarise research on the topic of roles. I firstly consider literature that considers the activity profile (level of participation) of CoP members and then studies of the functional nature of CoP members' roles. (Space does not permit a detailed review of research into specific CoP roles, such as the facilitator.)

\subsection{Core and peripheral members}

CoPs are known to have different levels of participation in terms of communication and involvement. There is typically a small leadership group, a core group of active members, and a larger group of peripheral members (Wenger, et al., 2002). Those in the core group identify topics of interest and help to move the group along the learning agenda (Wenger et al., ibid). Wasko and Teigland (2002) studied an online CoP of legal professionals using social network analysis. They found that the community's knowledge exchange was sustained by a critical mass of individuals ( $4 \%$ of members). This core group was not exclusive in that its members largely communicated with noncore members. The core group is described by Timbrell et al. (2007) as a key concept that characterises an online CoP. They argue that the essence of coreness is not simply about participation rates and requires further attention in research.

Wasko and Tiegland (2002) found a group of peripheral individuals who helped to sustain the CoP by infrequently posting responses to others. There was a further group of seekers who only asked questions. The role of the lurker has also received attention (Nonnecke \& Preece, 1999; Nonnecke, et al., 2004; Rafaeli et al., 2004; Soroka \& Rafaeli, 2006; Timbrell et al., 2007). A lurker is a member who does not post to the online CoP discourse, but may follow the online discourse, and may later become a more active member. The possibility of the lurker contributing to offline KT amongst non-active CoP members has not been investigated.

\subsection{Functional roles}

Fontaine (2001) proposes the following four categories of functional CoP roles, based on a synthesis of case studies. (This study does not distinguish between online and offline CoPs, but appears to assume that CoPs have an online dimension.)

(i) Knowledge domain roles are filled by people who "embody deep knowledge of the practice" (ibid, p.17). They include subject matter experts (SMEs), core community members, and members - the glue that holds a CoP together (ibid). 
(ii) Leadership roles are filled by leaders and sponsors who provide CoPs with legitimisation, visibility, strategic direction, and funding.

(iii) People performing three knowledge intermediary roles are responsible for facilitating the exchange of knowledge by capturing, codifying, retrieving and transferring it. These are:

Facilitators, who build a positive community dynamic, shepherd conversations and connect members with each other and experts. They "encourage and energize participation by interacting with the community, by endorsing ideas, and by directing knowledge to the appropriate experts" (ibid. p.19) Tarmizi and de Vreede (2005) examine this role in depth, outlining the facilitation activities needed at different developmental stages;

Content co-ordinators - the "ultimate" knowledge sources (Fontaine, p.19), and Journalists, who focus on capturing and codifying knowledge.

(iv) Support roles are filled by mentors, event co-ordinators and technologists.

2.4.2.4.3 Personality-based roles: Connectors, Maven and Salesman While Tarmizi and de Vreede and Fontaine emphasise the nature of activities performed by (mainly) appointed role-bearers, members' personality characteristics may also play a role in $\mathrm{KT}$. CoP roles have been characterised on this basis by Nichani and Hung (2002), who draw on Gladwell (2000), arguing the importance of the intermediary roles connectors, mavens and salesmen in online CoPs. In his bestseller, The Tipping Point, Gladwell (ibid) demonstrates how these roles can impact on social epidemics. Connectors are natural boundary spanners who have a gift for connecting people. Mavens are natural information specialists and brokers, while Salesmen are born charismatic persuaders.

\subsection{Roles and KT: A research gap}

There is an opportunity to investigate the relationship of CoP roles on KT. Thomson et al. (2005) applied Fontaine's roles in a discourse analysis study, aiming to investigate the relationship between roles and depth of learning. They contend there was a positive correlation between reported levels of learning, level of interaction and roles 
adopted. However, the research was inconclusive, with a low response rate and using a problematic analysis tool.

\subsubsection{Reconceptualising the online CoP}

Most research into online CoPs to date has been conducted in the context of 'traditional' online CoPs - private spaces, bounded by a shared online platform and using facilitated forums. However, recent technological developments and an emerging set of alternative CoP literature have challenged assumptions about what qualifies as an online CoP. The definition has not changed, but it is necessary to expand the parameters for inclusion.

\subsubsection{Online CoPs in blogging and discussion networks}

Efimova and Hendrik (2005), Hodkinson (2006) and Wei (2004) and Kaiser et al. (2007) have identified online CoPs in the high-density areas of reciprocal connections in blogging networks. In blog-based communities, discussions are neither bounded nor facilitated, but instead are distributed amongst the blogs of members who employ practices such as tagging, RSS feeding and social bookmarking, to monitor the conversation. Similarly, Murillo (2007) have identified online CoPs in the dense areas of Usenet discussion networks, screening them for a fit with Wenger's original CoP characteristics.

Awareness of these manifestations of the online CoP is very recent, so studies concerning the facilitators of KT have not yet been conducted in these settings. These recent developments suggest that there are alternative paths for the development of online CoPs: they may either arise as an extension of (co-located) organisational groups or develop in an emergent way from networks. Murillo (ibid) predicts that this will occur if a network of practice becomes more energetic and develops a coreperiphery structure. While the former kind of online CoP has a shared offline dimension, the latter does not.

\subsubsection{Castro's CoP ecosystem model}

Castro $(2004,2006)$ provides a further challenge to the conception of online CoP, proposing a more holistic, fully inclusive perspective. Building on the theme that different CoPs co-exist, with individuals belonging to more than one CoP (Hildreth et al., 1998; Wenger et al., 2002), he argues that any group of practitioners can now set out to shape practice - there is no single practice $(2004$, p.4). Secondly, he argues that with the Internet, costs of collaboration and information exchange have decreased, 
leading CoP members to communicate more frequently than before: "Now we can see more frequent interactions, hundreds of times more activity than in primitive communities" (p.5). This is coupled with a diminishing importance of face-to-face communication, and a less formal approach to written communication. Thirdly, there is greater information longevity, as the ability to store, find, and retrieve documents has increased, and as exchanges amongst members are readily codified. These factors have resulted in many more CoPs than previously.

In addition, online communication channels are now typically open, creating few barriers to access. CoPs therefore operate in a universal theatre, overlapping with other communities that deal with the same subject (p.6). The participation of individuals in more than one CoP within a field is common, so the domain is no longer the main boundary between communities. Based on these arguments, Castro proposes a model of a wide conversation space in which an individual may participate as a core member in some communities and in a peripheral way in others; the CoP Conversation Space Ecosystem (2004, 2006; see fFigure 4).

The model is a system containing a set of people and their conversations. Individual CoPs are "nuclei of activity within a wider ecosystem"(p.8). They take on different shapes and are differentiated primarily by their culture and set of information (ibid). Individuals select communities according to the suitability of the IT platform, the degree of fit with their learning needs, and the level of cultural fit. A key feature of Castro's model is that "information gathered in one place... may have an echo and reflection in several ways across the conversation space" (ibid, p.8). This suggests the possible existence, at system level, of a knowledge-amplification effect and a thematic convergence effect in conjunction with $\mathrm{KT}$.

Castro's arguments are convincing in light of the recent explosion of social technologies, such as the freely available Web 2.0 tools (for example, wikis, blogs, social bookmarking tools, and Twitter) and his work is significant in introducing the theoretical concept of an ecosystem of interconnected CoPs. The arena for interaction amongst professionals has, without doubt, increased dramatically in complexity since Lave and Wenger's discovery of CoPs, with such new online tools allowing for concurrent professional communication in diverse online contexts. Various questions of potential significance arise; for example, in what ways do these online contexts differ, how does this impact on their use, and how are they used in relationship to the CoP ecosystem as a whole? Castro's discussion of the model can, perhaps, be 
criticised for its failure to consider of the offline dimension(s) of the CoP ecosystem. If an ecological view of CoPs is to be adopted, it can be argued that this is a significant omission.

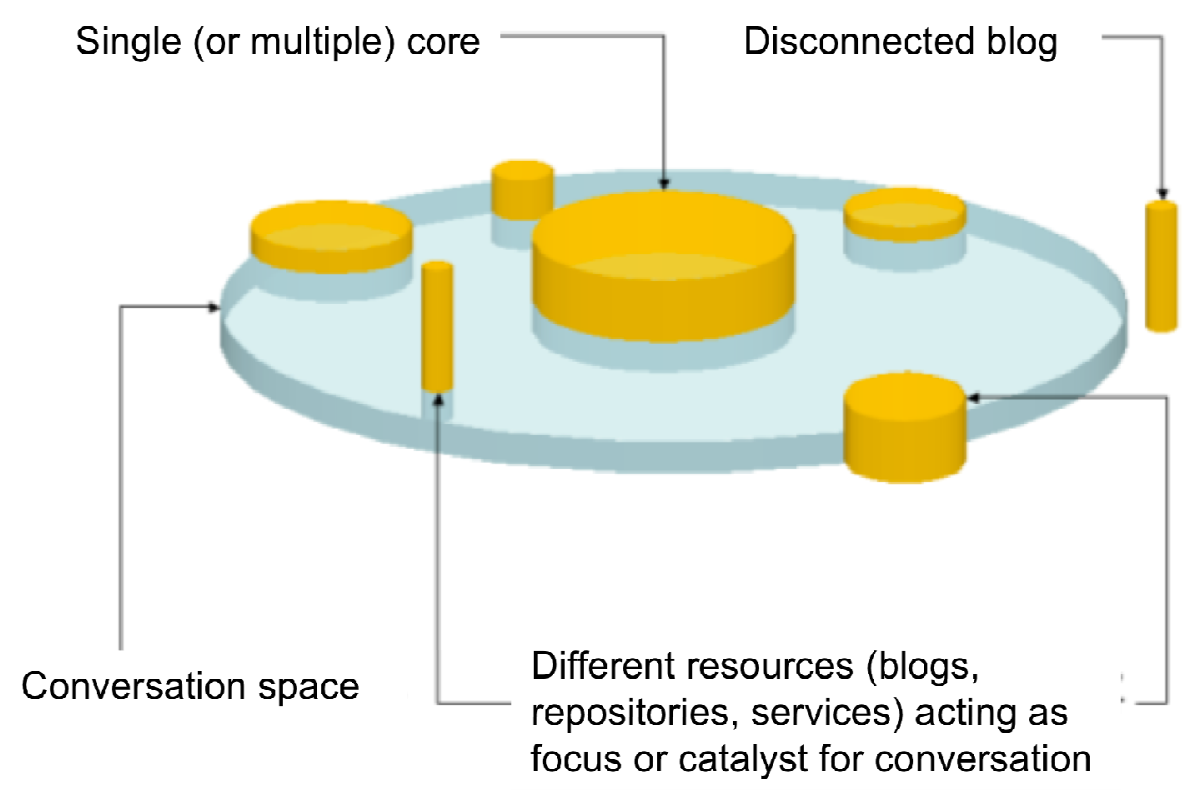

Figure 4: Castro's (2006) CoP conversation space ecosystem

Murillo (2008) has noted that, "for individuals, it will not be virtuality or co-location that matters most, but the fact that for different problems, different communities of practice are better resources, because of their area of expertise or because of the people that participate. Hence, people will likely seek membership in both co-located and virtual communities of practice and adjust their participation to fully exploit learning and identity-acquiring possibilities."

\subsubsection{The need for new CoP theory to explain KT}

Several authors have highlighted the lack of theory to explain how online CoPs work and the problem of assuming that Wenger's theory can be translated to fit the online context (Brown \& Duguid, 2000; Castro, 2006; Hargreaves, 2003; Zhang \& Watts, 2003). As Castro has noted, "the new observable creature does not fit well within the old theoretical framework" (2006, p. 3). In relationship to this study, it is questionable to what extent situated learning, a concept designed to explain the immersive face-toface process of professional knowledge acquisition in CoPs (Lave and Wenger 1991) 
is applicable in the online context. Where, from amongst the many available online and offline channels, should the professional be seen as being situated? Can a professional be situated in many places? It appears there is need for a new explanation of how professional KT occurs in the context of online CoPs. These are issues of significance to my research. This literature review has highlighted a lack of theory to adequately account for the process of professional learning or knowledge embedding ('deep' KT) in the context of online CoPs.

\subsubsection{Section summary: Online CoPs and knowledge embedding}

In this section, I have reviewed literature relating to how online CoPs may support KT and embedding. There is little understanding in this area. Although the complexity of human behaviour has been highlighted in relationship to the moderators of $\mathrm{KT}$, and the complexity of CoPs is recognised, this research does not acknowledge that $\mathrm{KT}$ itself may be a complex process, relying largely on an input-output view of KT. In the case of professional change, such a view may provide only part of the picture.

The review has highlighted a number of themes of potential relevance. These include the apparent suitability of new conversational technologies for supporting social interactions and therefore $\mathrm{KT}$, the suggestion that online CoPs may promote embedding through reflective practice, the possibility that differentiated social online contexts have a differing influence on $\mathrm{KT}$, and the suggestion that $\mathrm{KT}$ and embedding are more likely to occur during the middle and late stages of a CoP's development. There is also an opportunity to explore how particular roles (notably the knowledge intermediary or boundary spanner) function in relationship to the transfer and embedding of professional knowledge.

It is necessary to acknowledge that online CoPs have themselves undergone recent change. They have become more complex and more interconnected and have developed new manifestations, appearing in blogging networks. Castro's (2006) CoP Conversation Space Ecosystem model has been noted as worthy of investigation. Challenging assumptions about online CoPs, it portrays the online CoP setting in which today's professionals operate as a complex system of diverse online communication spaces. This is a far more complex system than the traditional online CoP model. Castro's description of the echoes and reflections that arise suggests a degree of thematic repetition and redundancy that may be conducive to the embedding of knowledge. His model is also significant owing to the potential number of boundaries 
involved across which KT may occur and it highlights the need for theory to explain how online CoPs facilitate KT and embedding. Lave and Wenger's situated learning theory seems unsuitable, due to the highly fractured nature of the online CoP environment, in which an individual may simultaneously participate in multiple online CoP contexts.

\subsection{Summary of literature review}

The NZ government has initiated a project to embed knowledge about effective teaching at system level. It relies on the transfer of knowledge via CoPs and online CoPs. However, there is a lack of research to suggest how this should occur and what issues are involved. My research addresses this gap head-on. In this chapter, I have summarised a range of theoretical and research-based literature of relevance to my research question.

In the first section I have outlined the transformational agenda infusing my research setting. Four issues are driving change in schools: (1) the government's focus on more effective and equitable teaching, leading to a system level imperative; (2) a global initiative to transform schooling to as to produce citizens with suitable competencies for a knowledge society (reflected in the transformed curriculum); (3) a view that IT can help facilitate change in teaching (in the 'right' direction); and (4) a vision of knowledge about what works in education as driving change. These converge to underpin my project.

The research reviewed in the second section indicates that in order for a KT and embedding strategy to be successful in the context of transformative professional change, it must take account of needs and issues that arise at all three levels: At the organisational, or meso level, knowledge embedding must account for (a) the stagedependency of the embedding process and its moderators; (b) the need to foster the ongoing social conditions that facilitate embedding, and (c) the need to build contextual structures that institutionalise the use of new knowledge. At the individual (micro) level, research in KM, education and cognitive science demonstrates unequivocally that embedding must allow for the individual reconstruction and personalisation of professional knowledge, including interpretive frameworks and beliefs, for adequate peer support, and for the building of cognitive congruence amongst peers, so as to foster absorptive capacity. Literature in KM, health and education shows that at the macro (system) level, it is necessary to consider the role of knowledge brokers and 
intermediaries, as well as external experts, in supporting individuals and organisations (Farkas et al., 2003; Hutchinson \& Huberman, 1993).

In the third section I have summarised literature relating to how IS and online CoPs might facilitate the transfer and embedding of knowledge. I have highlighted new developments in the manifestations in online CoPs, noting the implications of this for research. I have argued that there is a need for new CoP theory to explain how KT and embedding is facilitated through online CoPs.

The extant literature provides no clear answer to the question, how do online Communities of Practice (CoPs) facilitate the transfer and embedding of professional knowledge? The 'baseline position' could be summarised as follows: The embedding of knowledge (making new knowledge stick) is seen as essential to the knowledge transfer (KT) process, yet the way in which embedding occurs is poorly understood. Furthermore, the literature presents an apparent dichotomy, with embedding being presented by some researchers as a finite stage of $\mathrm{KT}$, and by others as an ongoing process. Both views appear useful, yet this dichotomy has neither been noted nor addressed. In addition, different challenges to knowledge embedding seem to exist at different level of analysis (organisation, individual, and system). This suggests a possible need for different knowledge embedding solutions at each level. Finally, within respect to how online CoPs might facilitate knowledge embedding we know virtually nothing: Despite the strong linkages between early CoP theory and the deep transfer of professional knowledge (Lave and Wenger, 1991), the online CoP literature is typically restricted to investigating knowledge sharing in lieu of actual knowledge transfer.

This project seeks answers to a strongly relevant research problem. For governments and organisations, there is abundant potential to capitalise on a better understanding of how online CoPs work. Massive investments have been being made in IT infrastructure, and there is both a growing awareness of the value of $\mathrm{KM}$ and a concern with using knowledge to drive system-level transformation. According to Fullan (2009), whole-of-system reform has come of age. This is also the age of the Internet. Owing to these factors, the recent evolution and widespread availability of online communication technologies, and the lack of online CoP theory, it is timely to address the identified research gap. 


\section{Research Strategy, Design and Method}

\subsection{Introduction}

In this chapter, I outline my research strategy, design and methodology. The aim of my research project was to generate theory that helps to explain a phenomenon about which little was previously known - how online Communities of Practice (CoPs) facilitate the transfer and embedding of professional knowledge. In approaching this question, I adopted an interpretivist epistemology. I used the case research strategy (Yin, 2003) and qualitative research methods. My approach to the generation of theory was inductive, based on a reflexive and iterative analysis process. The type of IS theory I set out to produce was explanatory (Gregor, 2006).

I begin by discussing the research paradigm then justify my chosen research strategy, design and methodology. Following this I describe the stages of the research process, my data collection and analysis methods and my approach to the generation of theory. I also discuss how I managed the assurance of quality and rigour in this project. Finally, I identify key issues and challenges that arose and describe how I managed these.

\subsection{The research paradigm}

\subsubsection{The philosophical paradigm in IS research}

A philosophical paradigm - the researcher's interpretive framework, or worldview enfolds any study in the Social Sciences. It guides the way in which the researcher conceives of their research objectives and approaches the research process, and it influences the nature of the research outcomes. Some authors have used the metaphor of a net to describe how the research paradigm operates. Bateson (1972) sees the researcher as being "bound in a net of...premises which - regardless of ultimate truth or falsity - become partially self-validating" (p.314).

Denzin and Lincoln (2000) see this net as having three dimensions: (a) the researcher's ontological perspective (what is the nature of reality?), (b) their epistemological views (what is the nature of the relationship between the inquirer and knowledge?), and (c) their methodological premises (how does one gain knowledge of the world?) (p.19). Because of the influential nature of the net, it is important for 
researchers to identify their research paradigm, relating it to the methodological literature, and acknowledging its role in framing and constraining their perspective.

\subsubsection{The ontological dimension}

The ontological dimension of the research paradigm (what is the nature of reality?) describes the type of belief system that underpins a research project. A realist perspective is one in which the external world is seen as made up of "pre-existing, hard, tangible structures which exist independently of an individual's cognition" (Fitzgerald \& Howcroft, 1998, p.319). A relativist perspective, on the other hand, is one in which the researcher believes that "multiple realities exist as subjective representations of the mind" (ibid, p.319). I have approached this research project within a relativist ontological view.

\subsubsection{The epistemological dimension}

The epistemological dimension of the research paradigm identifies the researcher's knowledge claim - their view on their own relationship with knowledge (Cresswell, 2003; Crotty, 1998; Denzin \& Lincoln, 2000). Traditionally, a dichotomy has been seen as existing between positivist research (positivism) and interpretivist research (interpretivism). Positivism is an approach derived from the physical sciences, based on the beliefs that the world conforms to fixed laws that can be explained by causality and that complexity is best managed by reductionism. Positivist epistemologies emphasise objectivity, measurement and repeatability (Fitzgerald \& Howcroft, 1998). Post-positivism, is a more recent epistemological view that recognises that a researcher cannot be completely positive about their claims on knowledge.

Interpretivism is based on the view that that there is no universal truth and understanding must therefore arise from interpretations created from within the researcher's frame of reference. Reality is seen as a subjective social product that is constructed and interpreted by humans, according to their beliefs and value systems (Myers, 1997; Orlikowski \& Baroudi, 1991). Research in this tradition aims to understand phenomena through the meanings people assign to them, and as meaning emerges through human complexity (Darke, et al., 1998; Kaplan \& Maxwell, 1994). According to Klein and Myers (1999), interpretive research is now an important tradition with the potential to produce deep insights into IS phenomena.

Myers (ibid) and Orlikowski and Baroudi (ibid) describe a third epistemological category, critical research, according to which knowledge is historically and politically 
constituted. (Creswell places critical research within the category advocacy/

participatory research.) The critical position sees knowledge as constrained by political, social and/or cultural domination. Research in this tradition is change-oriented and issue-orientated, encompassing feminist research, radicalised discourse, critical theory and disability enquiry.

There is some disagreement as to whether the three above epistemologies are necessarily opposed or whether they can be accommodated within the one study (Myers, ibid). Creswell's (2003) pragmatism, a pluralistic, problem-centred approach, allows for the pragmatic exchange or integration of perspectives. He sees it as a fourth epistemological option.

I adopted an interpretivist stance for this research and explain why in section 3.2.5.

\subsubsection{The methodological dimension}

The methodological dimension of the research paradigm identifies the researcher's views about how knowledge is best gained. Quantitative research methods are based around using measurements and statistics to test and validate theory, and to ascertain whether it can be generalised. Qualitative methods are designed to build a rich, deep understanding of a phenomenon as it occurs within a particular context.

Denzin and Lincoln (2000) view qualitative research as a situated activity that places the researcher in the world. They see the qualitative researcher as employing various interpretative practices to help make this world visible (ibid, p.3). This involves making sense of phenomena in terms of the meanings that people bring to them.

\subsubsection{Philosophical and methodological stance of this study}

I approached this study with an interpretivist epistemology. I saw this as a suitable philosophical approach for investigating the rich social interactions that may be occurring in online CoPs, and equally suitable for framing a study about the embedding of knowledge - known to be a subjective, complex and abstract phenomenon that cannot exist independently from its use by people. I saw interpretivism as providing me with the best opportunity for gaining deep insights into the research phenomenon, as convincingly argued by Klein and Myers (1999) and others.

My approach to data gathering and analysis was through qualitative methods. Because qualitative methods, unlike quantitative methods, focus on ordinary events in natural settings they are considered most suitable for revealing complexity and eliciting a 
depth of understanding (Miles \& Huberman, 1994). This is the goal if one is asking a how question. Qualitative methods are also seen as highly suitable for exploring a new area and generating conceptual frameworks (ibid, p.1). This is partly due to their flexibility: unlike quantitative methods they can be varied in the course of the study, according to the nature of emerging findings.

This flexibility proved beneficial to me in several respects. It allowed me to revise my interviewing technique to account for participants' understandings about online CoPs, eliciting more valuable data. More critically, it allowed me to adjust my research design in the course of data collection, so as to incorporate an additional online CoP: this nonofficial online CoP played a key role in the knowledge embedding process, but did not fit traditional descriptions of an online CoP. My choice of qualitative methods allowed me to revisit the literature and revise my initial conceptions about the nature of online CoPs, based on emerging definitions. I amended the study design and gathered further data to understand the role of this new CoP. Without this flexibility my study is unlikely to have led to the generation of multi-level theory.

\subsection{The research strategy: case research}

In addition to the research paradigm, the researcher must select a strategy of inquiry to frame and guide their research (Cresswell, 2003). I have used case research (Yin, 2003), a strategy that "investigates a contemporary phenomenon within its real-life context" (p.13). Case research is considered to be particularly well suited to the IS field, due to the fact that the focus of IS is not on technology per se, but on the relationship between IS and its organisational or social environment (ibid). Case research supports the field's aim to "understand the complex and ubiquitous interactions among organizations, technologies, and people" (Dubé \& Paré, 2003, p.598). It can also help researchers and practitioners to keep up with the rapidly changing nature of IS and organisations, suggest new lines of reasoning, and highlight opportunities, challenges and issues (ibid).

Case research strategy is seen as versatile and pragmatic (Dubé and Paré, ibid; Yin, ibid) in that it can be used in conjunction with either a positivist, interpretive, or critical philosophical perspective. It most commonly employs qualitative methods, but may also use quantitative research methods, or a combination. Yin (ibid, p.11) describes the ideal approach as whatever works best in relationship to a study's problem and research question. Due to fit with the IS field, its versatility, and its recently history of 
successful use, case research strategy has become well-respected and is probably the most-used research strategy in the IS field (Darke et al., 1998; Dubé \& Paré, 2003). This does not mean, however, that it would be a suitable for every study within IS.

\subsubsection{The case for case research}

According to Yin (2003), case research is the most appropriate research strategy when the researcher has little control over events, the focus of research is a contemporary phenomenon within a real-life context, and/or when how or why questions need to be asked. All three of these conditions were present in my study. Firstly, the events I investigated concerned the movement of knowledge within and between communities from which I was excluded and over which I had no control. Secondly, the context for the study was a contemporary phenomenon, a government strategy designed to transform teaching while fostering the use of ICT for effective teaching. Thirdly, the question guiding my study was a how question.

A further aspect of case research that made it suitable is the fact that it can be generalisable to theoretical propositions (Yin, ibid). Viewed from an interpretivist stance, theoretical generalisations should not be seen as wholly predictive for future situations, but rather viewed as tendencies (Bhaskar, 1979; Walsham, 1995). Walsham has adapted Yin's (implicitly positivist) concept of case research generalisability, proposing four types of generalisation that can arise from case research in the interpretive tradition: the development of concepts, the generation of theory, the drawing of specific implications in particular domains of action, and the contribution of 'rich insights' (ibid, p.79). My study resulted in all four types of generalisation.

Various sub-categories of case research strategy have been identified, including explanatory, descriptive, and exploratory (Yin, ibid). This study was explanatory in intent. It also had an exploratory flavour in that it sought to generate new theory, through a process of induction, in an area in which there is a lack of existing understanding surrounding the phenomenon being studied. As the literature review has demonstrated, no prior theory explains how knowledge is embedded in the context of the online CoP.

\subsubsection{Case design: a single, unique case}

The phenomenon being studied in case research must occur within clearly defined contextual boundaries (Miles \& Huberman, 1984; Yin, 2003). Case research may adopt a single or a multiple case design. Yin recommends multiple case design owing to its 
suitability for comparing and contrasting cases and for testing theory. However, he notes that a single case design is likely to provide the researcher with considerable richness in terms of events, people and data, leading to a depth of understanding (ibid). Yin sees a single case design as the most appropriate approach if the case is critical (i.e. it meets the conditions required for testing a theory), revelatory (based on a previously inaccessible situation), or unique in nature. In this study the selected case the ICT PD programme - was unique. However, it had a number of embedded subunits, providing an opportunity to capitalise on some of the advantages of a multiple case design.

\subsubsection{The case: ICT PD programme for schools}

The ICT Professional Development (ICT PD) programme was a government-funded three-year programme for NZ schools. It presented a unique opportunity in that one third of NZ schools were participating in it at the time of my research, grouped into around 100 self-selected clusters. I saw the programme as a suitable case for investigating my research question because of its system-level goal. It aimed to foster change in schools, with the goals of improving teaching and learning through the use of ICT, and strengthening CoPs through increased collaboration. There was also an implicit higher-level transformative goal behind the programme, as is evident in the following quote from the programme provider:

What we have seen in the (ICTPD cluster) programme to date is that we are not learning how to use ICT nor are we involved in developing ICT resources to match our curriculum. We are working on a new pedagogy for learning - looking at a new way of teaching... (Ham, cited by Cognition, 2007)

As a unique Case, the ICT PD programme met Yin's (2003) criteria for using a single case. It also provided a setting that was suitable for gaining a rich picture of CoPs because it supported a community-centric approach: Schools in NZ are self-governing and the MOE had fostered a 'grassroots' approach through which schools and clusters determined their own programme outcomes.

\subsubsection{Rationale for a single case design}

Although the ICT PD programme was unique, it would have been possible to approach this study as a multiple case, focusing the unit of analysis at the level of the cluster. This could have provided certain advantages, such as opportunities for comparative analysis and theory testing. However, I saw the benefits of the single case approach as outweighing these advantages: Viewing the programme as a single case allowed for 
consideration of the different system levels at which KT could potentially occur. I envisaged that KT would be occurring within schools and clusters, but also potentially between clusters within the programme. In other words, I felt that a single case would best support the possible emergence of a picture of both cross-boundary (cross-CoPs) and internal (intra-CoP) knowledge transfer and embedding. This was most likely to produce findings relevant to the government's concern with systemic level transfer.

In summary, I saw the single case approach as being richer and more suitable for investigating an issue of systemic concern. It allowed for the analysis of knowledge flows at several levels; within and across communities, and over time.

\subsubsection{The case structure}

In case research the unit of analysis may be at the level of an individual, an event, or an entity (Yin, ibid). In this case it was an entity: the ICT PD programme. I originally planned to include six school clusters (CoPs) as subunits of the case - three secondary and three primary, as well as national facilitators and the programme leader. School clusters were to be recommended to me by programme facilitators, based on their perceived suitability - i.e., their maturity as CoPs, which the literature suggested was likely to be indicative of KT activity, and their use of online communication amongst the teacher community - and their achievement of programme objectives. Based on data supplied by the MOE, I identified 20 clusters that would be in year 3 of the programme when I conducted my interviews in 2007, and a further 40 that would have recently completed the programme. This created a potential pool of 60 mature CoPs.

While I relied largely on the facilitators as intermediaries in locating suitable CoPs, a notice was also placed on my behalf, on a listserv sent to all programme clusters, inviting clusters in the third year of the programme with active online CoPs to participate. I was surprised to find that, despite the apparently large pool of candidate communities, it proved impossible to identify six suitable online CoPs within my timeframe: Several clusters had promoted their use of online CoPs to facilitators but were operating student-teacher communities only. In several cases, online CoPs turned out to be no more than resource banks. Other clusters that were keen to participate but were only just setting up online CoPs. After following a surprising number of false leads, I risked both project slippage and wearing out the patience of my business sponsors. I therefore embarked on the study with only four CoPs as 
subunits: two primary clusters (CoPs A and B) and two secondary school clusters (CoPs C and D).

I later included a fifth CoP - CoP E. This online CoP revealed itself only after I had begun interviewing. It was very different from the four cluster-based CoPs in that it had a highly distributed, open membership, no facilitator, and was based in a blogging network. It also had no formal association with any cluster, so was not associated with any organisational goals. CoP E was a valuable addition to this interpretative study owing to its complementary nature, its dynamism and its overlapping membership with the cluster-based CoPs. By adding it to the case I introduced complexity and reduced the equivalence of subunits, but this was more than compensated for by the depth of understanding it came to provide: I came to recognise it as a middle layer CoP that was instrumental in KT at programme level. The structure of the case is illustrated in Figure 5.

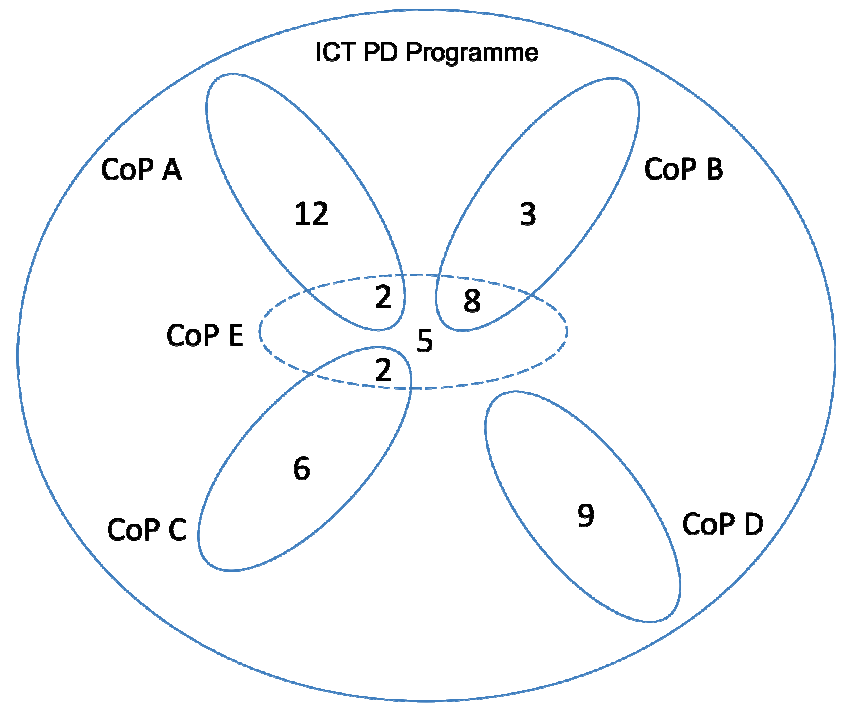

Figure 5: The case structure showing overlapping CoP membership

Despite the above issues, the sample included a wide geographical distribution of schools. Across the five CoPs, I interviewed 47 individuals based at schools in Southland, Otago and Marlborough in the South Island, and Wellington and Hawke's Bay in the North Island. A full case breakdown is provided in table 8 in Chapter 5.

\subsection{The role of the researcher}

The interpretive tradition recognises the researcher's role as central to the research process and results. The researcher must undertake "... the difficult task of accessing other people's interpretations, filtering them through their own conceptual apparatus, 
and feeding a version of events back to others."(Walsham,1995, p.77). Although there is no attempt to aim for "objectivity" or "neutrality", the researcher must acknowledge how their role as an interpreter impacts on data analysis, interpretation and the presentation of findings.

Walsham (ibid) identifies two different roles that can be played by the interpretive researcher: the outside observer and the involved researcher. Neither of these roles is viewed as an objective reporter, owing to the subjectivity involved in the collecting, analysing and reporting processes; and the possibility that the researcher may influence the interpretations of those being researched - the double hermeneutic (Giddens, 1987).

I adopted the outside observer role, as is typically done in case research (Walsham, ibid). This role required me to preserve some distance from the research setting and participants, which was not difficult. I had no personal stake in the interpretations or outcomes, and no formal alignment with stakeholders. This was an advantage, given that participants were relatively frank about their opinions. The disadvantage of this role was that I was unable to get a strong sense of the research setting from the inside. Walsham (ibid) has suggested that an outsider is unlikely to be privy to sensitive data, but I established good rapport with most participants and did not find this to be the case.

The wider setting for the study was the NZ school system, and the type of knowledge I studied was to do with teaching. Given this, the fact that I did not have a background as a teacher can be seen as both an advantage and disadvantage. It was a disadvantage to the extent that I did not have a ready understanding of the issues and concepts relating to teaching and its day-to-day challenges. I needed to be ever-alert for nuances, and to request validation of emerging understandings. (This was moderated by the fact that I had worked in the education sector - in publishing and website management - for five years, so was familiar with current issues and teaching terminology.)

My outsider perspective provided compensatory benefits. I believe that this role made it easier to see factors relating to the knowledge transfer and embedding processes (as distinct from teaching per se) and also factors unique to the cultural setting. My outsider's role also made it relatively easy to be open to emerging understandings of the teacher's role, as I lacked a strong personal opinion. I need to declare some bias in 
that I had an affinity for the goals of the ICT PD programme and saw it as a worthwhile programme.

\subsection{The research process}

My research process was iterative and flexible, in order to allow for the emergence and progressive confirmation of findings. Figure 6 provides a detailed outline of this process. I undertook four phases of data gathering and analysis.

\subsubsection{Phase 1}

The first phase (Data Collection/Analysis Phase 1) was focused on eliciting key issues, themes and categories in relationship to the research question. I gathered data (semi-structured interviews with teachers and principals, online data and programme documentation) from two CoPs and analysed this, creating a set of progressively refined interpretive categories leading to an emergent set of factors, processes and issues (see section 5.8). During this stage I iteratively refined my interview approach in line with teachers' understandings of key terms and the issues that were emerging.

\subsubsection{Phase 2}

In the second phase (Data Collection Phase 2/Analysis Phase 2) my focus was on exploring, teasing out and understanding the preliminary themes and issues in more depth, as well as identifying issues specific to primary versus secondary schools, leading to the emergence of theory. I conducted interviews with two further CoPs and the project leader, while conducting iterative analysis. During this stage, my view of the case became simultaneously clearer and less clear: On one hand, I gained rich and valuable new data from both CoPs. This enabled me to develop a preliminary theoretical model to explain the stages of the embedding process in schools: the knowledge embedding cycle. I also uncovered a series of contributing sub-processes facilitated by online CoPs. On the other hand, I found that key members of CoPs A, B and $\mathrm{C}$ belonged to a non-official online CoP that appeared to play a key role in facilitating KT at a national, or system level. Once this CoP became visible to me, I reviewed the literature, finding that my initial conception of CoPs had been too narrow - there were precedents for viewing this group of edubloggers as a CoP. I amended my case design to include CoP E and embarked on another round of data gathering and analysis, focused on understanding the role of this new CoP. The key outcome of including CoP E was that my perspective of the case structure had shifted firmly to a multi-level view: CoP E operated at a different level to the other CoPs. 


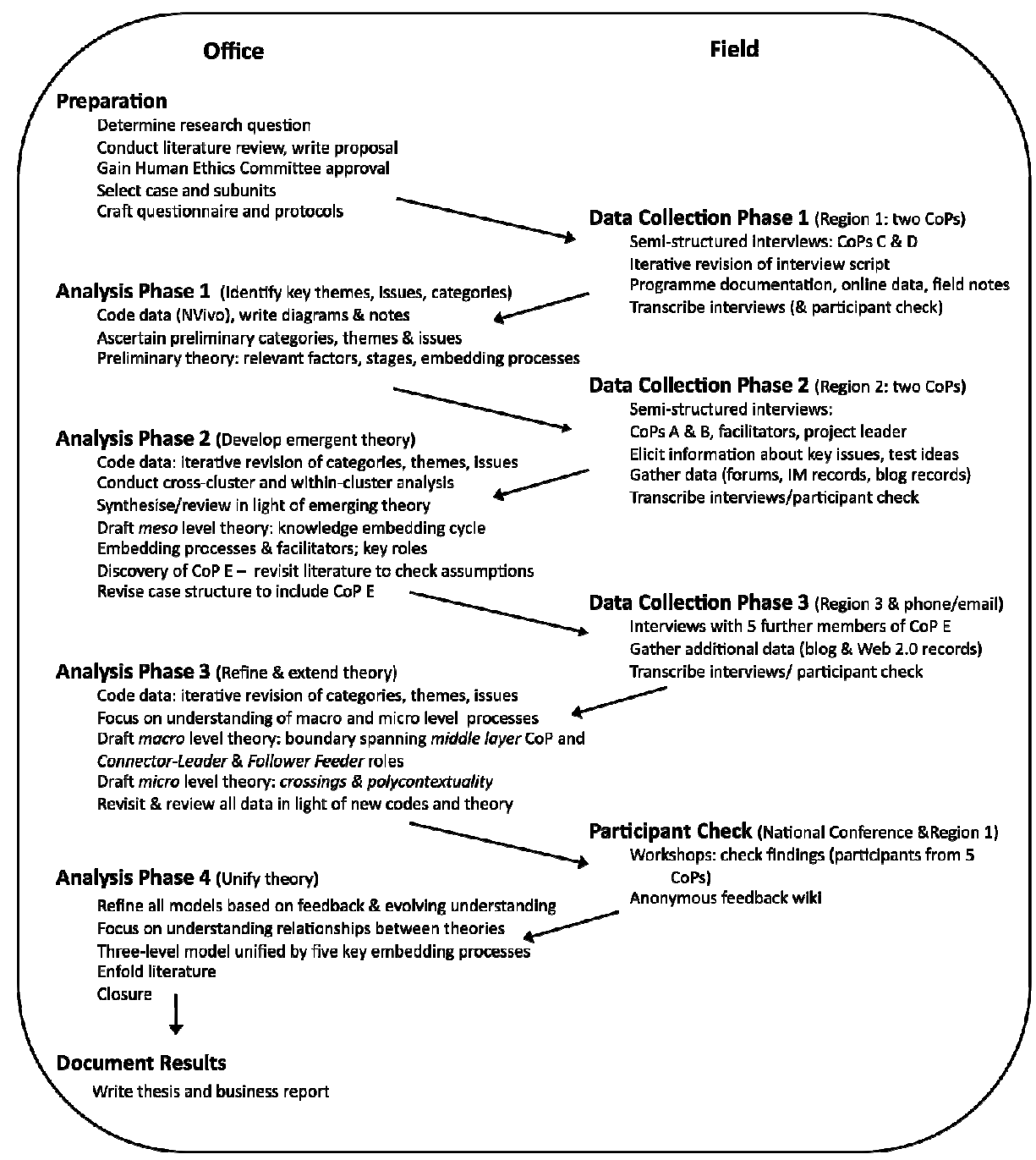

Figure 6: Overview of the research process

\subsubsection{Phase 3}

The third phase (Data Collection Phase 3 /Analysis Phase 3) was based around refining and extending my theory. I interviewed five additional members of CoP E and analysed these interviews together with extensive blog/Web 2.0 records. This led to the emergence of two new theoretical mechanisms through which online CoPs facilitated embedding process: a micro-level theory based around the themes of crossings and polycontextuality and a macro-level theory based around the role of CoP E as a middle layer CoP. The increasing richness of my findings during this stage were due to a combination of my increasing familiarity with the data, my growing interpretive sensitivity and - most importantly - the fact that CoP E was the most active online CoP of those studied: It was essentially a community of boundary-spanning individuals. This resulting expansion in theory at this stage provided me with richer answers to the how question, which is appropriate for an interpretive and inductive research process. 
It was now necessary for me to review previous data in light of my new understandings and to check the preliminary theory with participants.

\subsubsection{Phase 4}

In the final stage, my emphasis was on refining, streamlining and unifying the components of my theory. I conducted participant check workshops with self-selected members of all five CoPs, inviting these people to contribute anonymous feedback via a wiki (editme.com). This provided confirmation and feedback that allowed me to further refine the theory. I then reviewed critical data from all CoPs and conducted more analysis in light of (a) participant feedback, (b) my micro- and macro-level ideas and (c) the literature. This was a highly iterative and reflexive process. I reviewed new literature in cognitive science and psychology. Once I had reached theoretical saturation and the improvements became small, I moved on to finalising the theory and writing and reporting my results. The outcome was a three-tier explanatory theory.

\subsubsection{Data collection procedures}

Qualitative methods involve the collection of a range of empirical materials that describe "moments and meanings"(Denzin and Lincoln, ibid, p.3). A variety of materials can generate a deeper understanding of the phenomenon being studied by providing different views of the research phenomenon. The qualitative researcher must act as a bricoleur, piecing together an in-depth understanding of the phenomenon (ibid). A further reason for using multiple data sources was my desire to gain visibility of $\mathrm{KT}$ that might be occurring at different potential levels. I conceived these levels as being the individual $(\mathrm{I})$, the school $(\mathrm{S})$, the cluster $(\mathrm{C})$ and the programme $(\mathrm{P})$.

Creswell (2003) classifies qualitative data collection techniques into four groups: observations, interviews, documents, and audiovisual data (p.17). I used all of these techniques, apart from observations. My data sources, and their potential level of KT I saw each source as supporting, are summarised in table 5. (I have replaced Creswell's category audiovisual data with online records.) I used two main data collection procedures: semi-structured interviews and the harvesting of diverse online records. Interviews are considered a critical data source in interpretive research - they are the means through which the researcher can "best access the interpretations that participants have regarding the actions and events which...are taking place" (Walsham, 1995, p.78). 


\begin{tabular}{|c|c|c|c|c|c|}
\hline $\begin{array}{l}\text { Data } \\
\text { category }\end{array}$ & Details & I & $\mathbf{S}$ & $\mathbf{C}$ & $\mathbf{P}$ \\
\hline \multirow[t]{2}{*}{ Interviews } & Semi-structured interviews & $\sqrt{ }$ & $\sqrt{ }$ & $\sqrt{ }$ & \\
\hline & Members' check workshops & $\sqrt{ }$ & $\sqrt{ }$ & $\sqrt{ }$ & $\sqrt{ }$ \\
\hline \multirow[t]{11}{*}{ Online records } & Online forums & $\sqrt{ }$ & $\sqrt{ }$ & $\sqrt{ }$ & \\
\hline & IM records (Skype, ichat) & $\sqrt{ }$ & $\sqrt{ }$ & $\sqrt{ }$ & \\
\hline & Videoconferencing records & $\sqrt{ }$ & $\sqrt{ }$ & $\sqrt{ }$ & \\
\hline & Blogs & $\sqrt{ }$ & & & $\sqrt{ }$ \\
\hline & Wikis & $\sqrt{ }$ & & & $\sqrt{ }$ \\
\hline & Podcasts/ Teacher Tube videos & $\sqrt{ }$ & & & $\sqrt{ }$ \\
\hline & Listservs & $\sqrt{ }$ & & & $\sqrt{ }$ \\
\hline & $\begin{array}{l}\text { Web/ Web } 2.0 \text { records (RSS feeds, Twitter, Delicious tags, } \\
\text { blogrolls, blog \& web statistics) }\end{array}$ & $\sqrt{ }$ & & & $\sqrt{ }$ \\
\hline & Second Life records & $\sqrt{ }$ & & & $\sqrt{ }$ \\
\hline & Participant feedback wiki & $\sqrt{ }$ & $\sqrt{ }$ & $\sqrt{ }$ & $\sqrt{ }$ \\
\hline & Online conference and workshop records & & & $\sqrt{ }$ & $\sqrt{ }$ \\
\hline \multirow[t]{7}{*}{ Other documents } & My field notes/journals ( \& notes in Nvivo) & $\sqrt{ }$ & $\sqrt{ }$ & $\sqrt{ }$ & $\sqrt{ }$ \\
\hline & Milestone reports & & $\sqrt{ }$ & $\sqrt{ }$ & \\
\hline & Evaluations of the ICT PD programme & $\sqrt{ }$ & $\sqrt{ }$ & $\sqrt{ }$ & $\sqrt{ }$ \\
\hline & Presentations & & & & $\sqrt{ }$ \\
\hline & Strategic Ministry documents & & & & $\sqrt{ }$ \\
\hline & Participants' own studies & $\sqrt{ }$ & $\sqrt{ }$ & $\sqrt{ }$ & \\
\hline & Conference materials & & & & $\sqrt{ }$ \\
\hline
\end{tabular}

Table 5: Data types and their perceived suitability for revealing individual (I), school (S), cluster(C) and programme $(P)$ level KT and embedding processes.

\subsubsection{Approach to conducting interviews}

I conducted one-hour interviews with selected members of each CoP. Where possible I interviewed the principal, lead teacher and at least one other teacher from each cluster school. All participants were members of online CoPs, but with varying levels of activity. (Table 8 in Chapter 5 provides the participant breakdown.)

I followed a semi-structured interviewing approach, using open-ended questions based on a script (see Appendix A), and following up on issues as they were identified to elicit more information. I conducted early interviews with the aim of identifying broad themes related to the research question. As my data analysis became more advanced, I finetuned my interviewing approach to hone in on key issues. My interview tactics were a key interpretive tool in this study. In Chapter 4, I discuss how I iteratively developed these tactics and how I used them to address specific research issues. 
Good technique alone is not sufficient for good interviewing. It needs to be supplemented by strong social skills, a high level of personal sensitivity, and nonjudgemental listening (ibid, p.78). I aimed to be conscious of this and to critically evaluate and improve my performance, avoiding over-direction (Walsham, 1995). The interview data I collected was personal, anecdotal and reflective.

I recorded the interviews on tape and transcribed them as soon as possible (after transcribing fifteen, I engaged a professional to assist). After transcription, I sent the transcripts to interviewees to check for accuracy, also inviting them to elaborate further. (I used an Excel spreadsheet to keep track of this process.) I incorporated participants' amendments and annotations into the transcripts prior to analysis. Following interviews, I gathered material such as programme documentation and online forums.

\subsubsection{Data analysis procedures}

When analysing qualitative case research data, it is important to recognise that the data collected is soft, reflecting individuals' reported experiences, and (in the case of field notes and interview questions) the researcher's interpretation. Methodological rigour in case research arises from, among other things, appropriate data analysis techniques. The data analysis process in a qualitative study is seen as involving three overlapping sub-processes: data reduction; data display; and conclusion drawing and verification (Huberman \& Miles, 1998). Data reduction involves systematically reducing the data into categories; summarising, coding and identifying themes. Data display requires the organisation of information so that actions can be taken and conclusions drawn. Conclusion drawing and verification is how the researcher interprets and draws meaning from the displayed data.

Eisenhardt (1989) notes that in the case of interpretive case research, there must be an iterative data analysis process involving continuous interplay between data collection and analysis (data reduction). This is what enables the researcher to remain flexible and to make adjustments to data collection, improving the prospects of generating quality theory. Huberman and Miles (1998, p.181) provide a model to illustrate this interactive and iterative process, reproduced in figure 8 . This exhibits the approach I followed. I discuss how I applied a reflexive analytical approach in addressing key aspects of my research problem in Chapter 4, using the metaphor of an interpretive toolkit. 


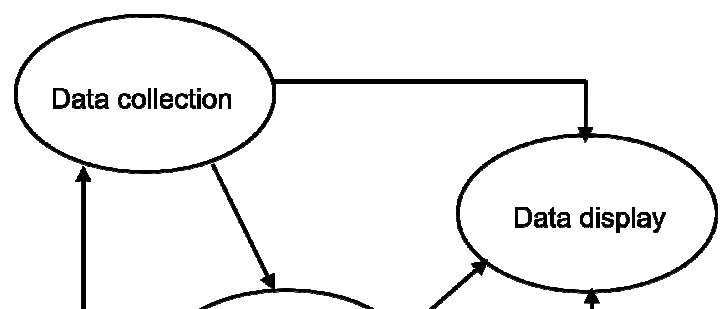


data, following Lincoln and Guba's (1985) recommendations for bridging, filling in, extending, and surfacing. I aimed for the codes to be semantically close to the terms and concepts they represented, have structural relationships to each other, and to be appropriate for the level of detail or unit of analysis that they described (such as a sentence, word or other or sub-unit of text). My concern for strong contextual relevance is reflected in my selection of names for the stages of the knowledge embedding cycle (see Chapter 6). These self-explanatory descriptors adopt a journeying metaphor that was in common use amongst participants: Plotting the Course, Coming on Board, Setting Out, Staying on Course, Anchoring and Settling. When I ran participant check workshops, I found that participants quickly identified with the early versions of this model.

I undertook coding as soon as possible after each round of data gathering, in order to keep an up-to-date overview of the key themes and issues. As the issues and codes evolved, I changed the interview questions accordingly, allowing for focusing in on key ideas, and refining and teasing out of emerging theory. As Miles and Huberman recommend, I worked with "loosely held chunks of meaning", unfreezing and reconfiguring codes as required by emerging meanings (ibid, p.70).

Once I had assigned a reasonable number of codes to the emerging data, I applied pattern-matching logic to identify emergent motifs or patterns. This involved grouping descriptive and interpretive codes (identified themes and issues) into higher level encompassing theme, identified by a set of pattern codes - explanatory or inferential codes (Miles \& Huberman, 1994, p. 70). For example, I used the early pattern codes roles, facilitators of embedding, key metaphors, embedding process and types of knowledge.

Whenever a new code emerged as being salient, I needed to return to the interview transcripts and reinterpret data with this new code in mind. (This happened, for example, when I developed the micro- and macro-level views of how embedding was occurring. It was like applying new lenses to the data.) NViVo is a very useful tool for this kind of layered coding, as one is able to recode without uncoding.

Over time I became deeply acquainted with my data and was readily able to recall key phrases from interviews and online records. At this point, search tools installed on my laptop (Google and, more recently, Spotlight) became useful. Rather than having to hunt manually inside existing NVivo codes, these tools allowed me to type in the 
remembered phrases and rapidly identify their source prior to coding. (I found that NVivo was most useful for around the first $75 \%$ of the analysis process but after this became a slight barrier. My attitude was perhaps influenced by my having suffered two corruptions of the NVivo database.) From time to time I also referred to annotated and highlighted hard copies of the data.

As the study progressed, the data collection and analysis process became more iterative, and I began to interpret and reinterpret data in the light of emerging and preexisting theory. Eisenhardt (1989) has noted that "analyzing data is the heart of building theory from case studies, but it is also the most difficult and the least codified part of the process" (p.539). Many of her recommendations for analysis were relevant to this case and informed the approach to data analysis at a structural level. For example, in the latter stages of the research process, I conducted within-case and cross-sub-case analysis. I determined unique patterns for each CoP prior to seeking general patterns across the programme. Cross-CoP analysis involved me in searching for patterns and categories, and then testing them by juxtaposing other CoPs and looking for similarities and differences.

\subsubsection{Data display}

In addition to text and tables, I have used a range of data display techniques in presenting the data, with the aim of making ideas and data more accessible. These include various kinds of diagrams, including mindmaps, flowcharts and structural models.

\subsubsection{Conclusion drawing}

The final stage of data analysis is the drawing and verification of conclusions. The key activities were (a) the development of new theory and (b) verification of the findings assuring quality and rigour. I consider each of these in turn.

\subsubsection{Development of theory}

Gregor (2006) has proposed a taxonomy of five types of inter-related theory in IS: (1) theory for analyzing, (2) theory for explaining, (3) theory for predicting, (4) theory for explaining and predicting and (5) theory for design and action. In this study I have aimed to produce theory for explaining, in the form of primary constructs and statements of relationships between the constructs, with the aim of being both "new and interesting" and explaining "something that was poorly or imperfectly understood 
before" (p.624). Owing to the fact that very little is known about the phenomenon being studied, it is appropriate for the sub-type of theory produced to fit Gregor's "lower-level" category of theory for explaining, whereby "explanations are given for how and why things happened in some particular real-world situation" (ibid, p.624). Gregor notes that interpretive field studies are an appropriate approach for developing this type of theory (ibid). This provides further support for my selection of a case research strategy.

In the course of data analysis, as themes, concepts, and the relationships between concepts began to emerge, I followed a systematic approach to theory induction. This involved ongoing construct identification, as I compared data with emerging theoretical constructs. I made many successive refinements with an iterative movement towards a final theory - in this case a three-level theory - that fitted with the data. (I have outlined this process in sections $5.1-5.4$.)

A critical aspect of my theory development process was comparison of emerging results to the existing theoretical literature. This is described by Eisenhardt (1989) as the enfolding of literature. Juxtaposition of emerging theory with conflicting literature is seen as beneficial in that it forces a more creative, frame-breaking mode of thinking (p.544). This can lead to deeper insights concerning both the emergent theory and the conflicting literature. It may also help clarify the limits of generalisability of the new theory. (An example of this is my realisation that the theory of situated learning associated with traditional CoPs could not account for how online CoPs facilitated the embedding of knowledge in the study setting: Participants crossed between many different online and offline contexts in the course of a day's work, becoming constantly re-situated.)

Comparison with similar literature is also important in that it can link similarities in phenomena that are not normally associated. This can result in a theory of a higher conceptual level (ibid, p.544). My theory regarding the micro-level facilitating mechanism of crossings is a result of insights I gained by juxtaposing the concept of polycontextuality from research into expert cognition (Engeström, et al., 1995; Goodwin, 1990; Reder, 1993) with my findings regarding multiple engagement spaces.

Figure 8 shows the broad approach I followed in inducting theory (Cresswell, 1994). 


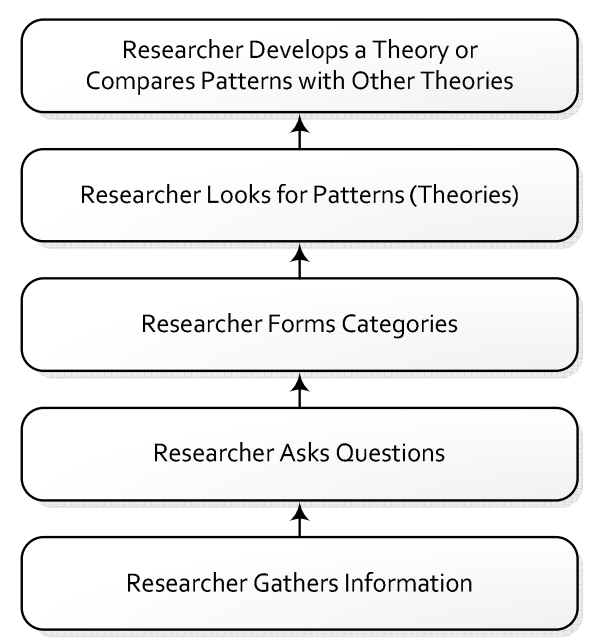

Figure 8: The inductive mode of research in a qualitative study (Creswell, 1994)

Closure in terms of theory development is ideally reached when "theoretical saturation" occurs (ibid). This means that the incremental improvement of the ongoing iteration process reaches a point where it is deemed to be minimal. A further pragmatic factor influenced my closure of theory development: my doctoral study was conducted as a project, with a planned end date.

\subsubsection{Approach to ensuring quality and rigour}

Eisenhardt (1989) outlines a much-cited process for building robust theory from case research. I followed her approach, in that my research process was iterative and tightly linked with empirical data. However, Eisenhardt's guidelines are underpinned by a positivist view. This is an interpretive study, so it is necessary for me to consider the issues of quality and rigour from an interpretivist stance. The issue of quality assurance in relationship to interpretive and/or qualitative methods is often raised. This is due, in part, to the fact that the positivist concepts of validity and reliability cannot be readily translated into interpretivist terms. Data, in the interpretivist paradigm, is inherently different from the positivist view of data: It is inseparable from the researcher's views, the interplay of these views with those of the research participants, and the research context. In other words, the interpretive researcher not only interprets, but may influence data that is gathered (Klein \& Myers, 1999; Lincoln \& Guba, 1985).

Numerous possible pitfalls have been identified in relationship to interpretive studies. These include the concern that a researcher's adoption of an interpretive stance will not guarantee collection of valuable data (Walsham, 1995), the risk of missing subtleties of human interpretation, a concern that premature, or narrow, use of theory 
by the researcher may lead to narrowing of interpretation, and the risk of researchers influencing participants' understandings through their use of language and concepts (ibid). Interpretive researchers face a challenge in that not only are they seeking to understand a moving target in the form of real-world phenomena (Klein and Myers, 1999, p.73); they must also communicate with a moving target (Sarker, 2007, p.251): audiences are at varying stages of an evolutionary continuum with respect to their understanding of qualitative research.

I now discuss how I tackled quality assurance issues with reference to two wellestablished sets of criteria: Lincoln and Guba's (1985) criteria for trustworthiness and then Klein and Myer's (1999) Seven Principles for Interpretive Research in IS.

\subsubsection{Trustworthiness}

A number of authors see the ensuring of trustworthiness as the analogue of reliability in interpretive studies (Denzin \& Lincoln, 2005; Golafshani, 2003; Lincoln \& Guba, 1985; Mishler, 2000; Seale, 1999). Seale states that the "trustworthiness of a (qualitative) research report lies at the heart of issues conventionally discussed as validity and reliability" (p. 266). Trustworthiness is a measure of rigour relating to the value and authenticity of findings. According to Lincoln and Guba (ibid), the key question trustworthiness addresses is: "How can an inquirer persuade his or her audiences that the research findings of an inquiry are worth paying attention to?" (p. 290). They propose a set of four criteria for addressing trustworthiness; credibility (replacing internal validity), transferability (instead of external validity), dependability (instead of reliability) and confirmability (instead of objectivity) (ibid, p. 300). My approach to managing these criteria is outlined below.

\subsection{Credibility}

Credibility arises from the richness of the information gathered and the analytical abilities of the researcher (Patton, 1990). The techniques I used for enhancing credibility were running member checks and a feedback wiki, inviting participants to corroborate and comment on my preliminary findings (Lincoln \& Guba, 1985), using an appropriate, consistent interviewing approach, developing results that have structural coherence, documenting the recursive nature of the research data gathering and analysis process, and ensuring the adequacy of references (Krefting, 1991). Further credibility arose through the application of my university's code of human ethics. 


\subsection{Transferability}

The transferability of a theory depends on the degree of similarity between the original situation and that to which it is being transferred. The qualitative researcher cannot predict the transferability of findings, but is required to provide sufficient information to allow others to determine whether the findings might be applicable to a new situation (Lincoln \& Guba, 1985). I aim to do this by providing contextual information and documenting considerations and qualifications surrounding my findings.

\subsection{Dependability}

Lincoln and Guba (ibid, p.317) propose the use of a third party inquiry audit as a means for enhancing the dependability of qualitative research. This is inappropriate for a $\mathrm{PhD}$, so as far as possible, I have applied Krefting's (1991) techniques. Krefting suggests that dependability is improved through dense description of research methods, stepwise replication, triangulation, and the code-recode procedure. In crosschecking and illustrating findings, I have triangulated different types of data (interviews, documents, online records). This reduced the opportunity for misinterpretation, identified areas of tension for further exploration, and increased the richness of understanding. Dependability also arises from some stepwise replication within my case - there were four structurally similar subunits (CoPs $A, B, C$ and $D$ ).

\subsection{Confirmability}

Confirmability refers to the degree to which the researcher can demonstrate how the research interpretations have been reached. As Lincoln and Guba (ibid) recommend, I have kept an audit trail comprising data, analysis notes, synthesis products, process notes, memos and developmental ideas (ibid, p.320-321).

\subsubsection{Klein and Myer's principles for interpretive research}

Some authors see it as unsuitable to use generic quality assurance criteria for all qualitative research, focusing instead on assurance guidelines for specific methods. Of particular relevance to this study is Klein and Myer's (1999) seven fundamental principles for conducting and evaluating interpretive research in IS. The principles, derived from hermeneutics, phenomenology and anthropology, are seen as a way to improve the "plausibility and cogency" of interpretive studies (ibid, p.79). I have followed these principles, as outlined below.

\subsection{The hermeneutic circle}

Klein and Myers view understanding as best constructed through an ongoing alternation between considering the interdependent meaning of a whole and its parts, as the seven principles are applied (ibid, p.72). I used this approach in the iterative 
generation of my three-level theory (outlined earlier in this chapter) and in the reflexive development and use of an interpretive toolkit to build understanding. I discuss my use of this metaphorical toolkit in the next chapter.

\subsection{Contextualization}

Klein and Myers emphasise that the researcher must "critically reflect on the social and historical context of the research setting, so that the...audience can see how the current situation being investigated emerged" (p.72). I have done this in Chapter 1. I refer again to the significance of the study context a number of times in this thesis. A converging set of strategic drivers provided a motivating force underlying the phenomenon under study.

\subsection{Interaction between researcher and subjects}

The third principle is the need to critically reflect on how the research materials are socially constructed through the interactions of the researcher and participants. It is necessary to acknowledge the role of participants as interpreters and analysts, and their impact on the research data (ibid, p.72). I have kept this in mind; for example, in managing teachers' conceptions regarding what an online CoP is, and in running participant checks through workshops and a feedback wiki (see Appendix C).

\subsection{Abstraction and generalisation}

The fourth principle is the need to relate theoretical concepts and abstractions to specific findings from the data (ibid). This enables the reader to follow the process through which theory is developed. I do this in Chapters 5,6,7 and 8.

\subsection{Dialogical reasoning}

The fifth principle involves having sensitivity to contradictions between theoretical preconceptions guiding the research design and the findings, and addressing these (ibid, p.72). My discovery of CoP E - an online CoP that sat within a blogging network - reflects my application of this principle. I needed to revisit my conceptions about what qualified as an online CoP in order to recognise its existence and the fact that it played a key role in embedding knowledge at system level across the case. I aimed to apply this principle more generally.

\subsection{Multiple interpretations}

The sixth principle is the need to be sensitive to, and address, the different interpretations of research participants (ibid). I have aimed to do this throughout my commentary. 


\subsection{Suspicion}

The final principle is the need to be suspicious of possible biases and distortions in the narratives of participants (ibid, p.72). This issue arose for me when some teachers described having made a sudden decision to commit to change, but the facilitators involved stressed that this was a rare situation. My explanation for this difference draws on research into the nature of human memory; notably problems to do with temporal recall. (I suggest that teachers may have collapsed time in retrospect.) Beliefs and values are strongly interwoven with my data, as they are known to play a key role in professional change. I have identified persuading as a driver of embedding and was sensitive to instances of this.

Klein and Myer's principles guided my overall interpretive approach to this project and I used them for periodic 'health checks'. Together with Lincoln and Guba's criteria for trustworthiness, they provided a robust reference framework for the pursuit of quality.

\subsection{Issues and Challenges}

This section outlines key issues and challenges that I faced, and how I managed them.

\subsubsection{Access to organisations and participants}

Gaining access to the ICT PD programme and participants was critical to the success of my study. I reached an agreement with the MOE (the sponsoring organisation) regarding expectations on both sides. The MOE provided a letter indicating support of my project to the schools involved. This enabled teachers to be granted release time to attend interviews. I established goodwill with stakeholders in each cluster and school, making it clear that I was not representing the MOE.

I have previously discussed the problem I had in locating suitable online CoPs. My approach to this involved building relationships with national facilitations who acted as go-betweens in locating clusters and allowed me to place a request on the national listserv. The problems I encountered may reflect a lack of knowledge about which clusters used online CoPs, a low uptake of online communication tools, and/or limited conceptions about what constitutes an online CoP. (In future I would ask for clusters where teachers "use online tools for regular communication with each other.")

\subsubsection{Constraints on the data gathering period}

Schools are sometimes unwilling to release teachers for participation in external projects in the final term of the school year, due to exams and end-of-year 
commitments. This placed constraints on my project timeline. My data gathering began in February 2007 and ended at the end of the third term 2007. I adopted a project management approach to ensure that the project remained on time. It would have been possible for my interviews to continue into the 2008 school year, but this was undesirable given the problem of sustaining relationships with participants. Turnover of staff is high in the schooling sector.

\subsubsection{Bias in data sources}

The issue of bias has already been discussed with respect to my role as a researcher. In qualitative research, personal opinion is inevitably bound up with data sources. The view of any one individual is unlikely to dominate, given the number of participants whom I interviewed (47). When interviewing the business owner and national facilitator, I took care to capture contextual issues relating to these people's interests and objectives. Triangulation of a range of data sources helped me gain a balanced approach.

\subsubsection{Richness of information}

In interpretive research it is vital to ensure richness of data sources. I designed my interview approach and questions to elicit maximum richness. I also took an iterative approach to interviewing, refining my interview script and tactics in order to maximise the richness and relevance of responses. I aimed to structure questions so they were relevant to the research question while allowing room for participant improvisation (see Chapter 4). I also harvested a range of complementary data sources.

\subsubsection{Recognising embedded knowledge}

A key issue that arose during the data gathering stage was how to 'recognise' knowledge that was being (or had been) embedded, and how to help participants do this, so as to elicit relevant interview content. In the next chapter, I discuss in depth how I tackled this.

\subsubsection{The need for a 'knowledge focus'}

Professional knowledge is a very broad concept, potentially encompassing any topic. Unless I could determine what professional knowledge to study the embedding of, my study risked being dangerously unfocused. Studying too broad a blend of knowledge could create entanglements of understanding, impacting on the usefulness and transferability of my results. Perhaps, for example, different mechanisms were involved in the embedding of different kinds of knowledge, creating too much complexity. For 
these reasons I determined that I needed to establish a clear 'knowledge focus' for the study. This would also help me know what to look for in terms of embedded knowledge. In the next chapter, I outline how I tackled this, establishing the new way as this study's knowledge focus.

\subsubsection{Timing and co-ordination challenges}

Owing to my iterative approach and travel logistics, my data gathering needed to occur between February and October 2007. I interviewed participants in the first two CoPs early in the school year, and interviewed those in the other clusters later in the year, when the embedding of knowledge was probably more advanced. However, seeing clusters at different stages of the embedding process and school year was beneficial, helping me to appreciate the challenges involved at different stages.

\subsubsection{Ethical issues}

I have followed appropriate ethical means of data collection, analysis and reporting. Ethical assurance measures include: (a) Informed consent from participants; (b) the interview transcripts being made available for participant checking and signoff, (d) emerging findings being made available to participants for feedback and final findings being made available upon completion; and (e) an agreement made that the privacy and confidentiality of individuals and organisations would be protected. This study was approved by Victoria University's human ethics committee. Relevant documentation appears in Appendix A.

\subsection{Presentation of results}

The results of this study have a diverse potential audience, ranging from academics in IS and Education, to business and/or government-based practitioners such as strategists and policy makers, knowledge workers, consultants, and senior managers. Bearing in mind that these groups (and individuals) will have different communication preferences, I have aimed to present the results of this study in a narrative way that is accessible to all these audiences. Where possible, I have used a narrative style and some rhetorical colour. The strengths of using written narrative are its familiarity, its ability to convey precise information, and its suitability for explaining abstract concepts (Yin, 2003). I have included quotations to illustrate the affective, personal nature of participants' responses to issues, add interest through diverse voices, and substantiate abstract concepts, while grounding them in everyday human contexts. I have complemented the written narrative with visual methods of communicating the results. 
Nonetheless, my style is constrained by the limitations of the thesis as a genre. The needs of other audiences can be met, to a degree, by re-versioning, synthesising and reducing the content. My results are positioned at three levels, which can be seen as fitting the interests of different audiences. For example, leaders and facilitators might be more interested in the meso- and macro-level findings; and individual practitioners in the micro- and macro-level ones. Central agencies may be more interested in the whole picture. There are future opportunities to slice and dice my results and present them as required for relevant audiences. (For example, I have written a separate business report, using the conventions of business style, for government stakeholders.)

\subsection{Summary: Research strategy, design and method}

In this chapter, I have outlined and justified my research paradigm, method and design. I have also justified the choice of a single case design and shown how my iterative data gathering and analysis methods led me to develop a three level explanatory theory. I have also addressed how I handled quality assurance and I have outlined my approach to presentation of the research results.

I conducted this research project with an interpretivist approach, using case research strategy, qualitative methods and the iterative induction of theory. This was an appropriate strategy for tackling my research topic, which was based around a how question in an area in which there was a significant lack of established theory: How do online CoPs facilitate the transfer and embedding of professional knowledge?

The case for the study was a national programme that aims to integrate ICT into teaching, in a way that contributes to 'effective' practice. The programme relies on online CoPs to assist in the transfer and embedding of knowledge, within and between a series of school clusters. It was a single (unique) case with a series of embedded subunits - a selection of mature CoPs.

My research method was iterative and reflexive. It depended on a versatile semistructured interviewing approach, together with the application of a flexible, but critical interpretive and analytical lens. The large quantity of data I gathered made this a challenging, but rewarding process. 


\section{Recognising Embedded Knowledge}

"What we see depends mainly on what we look for" - Sir John Lubbock

\subsection{Introduction}

In order to be able to ascertain how professional knowledge was being embedded in my research setting, I needed to understand what professional knowledge was involved: What kind of professional knowledge was significant in the research context? What should I be looking for? And what should I not be looking for? This chapter outlines this issue and how I addressed it. It describes how I established a knowledge focus for the study, pinpointing the identity, or essential nature, of the professional knowledge that was being embedded, and that mattered most in the study context. The chapter also introduces the key sub-themes and dimensions of this knowledge. By identifying a knowledge focus that was grounded in the research setting, supported by the data, and meaningful to me as a researcher, I gained a valuable interpretive filter and provided the project with clearer relevance, coherence, and manageability.

This chapter is also about the challenges that I faced in studying the amorphous concept of embedded knowledge. As can be expected of an interpretivist researcher using an inductive approach, my early understanding of the knowledge that was being embedded in the research context was less than clear. I discuss the key issues that I faced in identifying this knowledge and in implementing my research question, and how I developed an interpretive toolkit to help me tackle these challenges. This metaphorical toolkit comprised the knowledge focus, a set of interview tactics, and an evolving mental model of the dimensions of the knowledge that was being embedded. I developed these interpretive tools in a reflexive, and reflective, manner over time.

This chapter provides an important foundation for the chapters that follow. It explains key concepts which evolved alongside, and which influenced, my collection and interpretation of research data; and it establishes clear parameters for the relevance of my findings, putting a stake in the ground with respect to the complex landscape of literature on the subject of professional knowledge. Perhaps most importantly, it positions my study as being about the process of embedding new knowledge in the context of transformative professional change. 


\subsection{Embedding what? Identifying a knowledge focus}

From the outset of this study, I felt that in order to meaningfully investigate how professional knowledge was being embedded in the context of the ICT professional development (ICT PD) programme, I would need to gain an understanding of the knowledge involved: What kind of professional knowledge was being embedded? What was it about? I saw this as important for several reasons.

There is an extensive literature on professional knowledge, including research about teachers' knowledge, characterised by diverse ideas about what constitutes professional knowledge and how best to conceptualise it (Munby, et al., 2001). Given this landscape of complex, competing theory, I believed it would be important to identify and understand the nature of the knowledge involved in my study, to determine how best to interpret it for my research purposes, and to be able to justify this interpretation as a means of contextualising my findings. Perhaps, for example, different kinds of professional knowledge involved different embedding processes and different sets of issues. More importantly, I hoped that by answering this question I could establish a sense of purposeful focus - a core knowledge theme - to help me to 'see' and track the process of knowledge embedding in my research setting.

The ICT PD programme's objectives were broad ranging. Its intent, outlined in agreements with schools, was to increase 'teachers' ICT confidence, skills and pedagogical understandings of ICTs, increasing administrative efficiency in schools, fostering quality learning communities, increasing the frequency and quality of the integration of ICTs to support effective classroom teaching and learning and improving student achievement" (Ham, 2008). Was there a way I could narrow this potential grabbag of knowledge down to provide a meaningful focus for studying the embedding process? The Chinese proverb, "If you chase two rabbits, both will escape" underlines the risk of having a scattered focus.

My desire for a knowledge focus arose in part from the diversity of professional knowledge, and in part from my prior experience as a researcher. I had undertaken an earlier study investigating the process of inter-organisational knowledge transfer, in which the knowledge being transferred had been well defined. My ability in that study to focus on a particular knowledge theme, a new strategic model for measuring government success, meant that my discussions with research participants were straightforward. It also made it relatively easy for me to follow the 'progression' (or non- 
progression) of knowledge through the participating organisations and to screen out irrelevant material. In the absence of a knowledge focus for the present study, how would I track the process of embedding? I was concerned about the study's potential to become a series of fuzzy discussions, producing a proliferation of hard-to-interpret data.

Any knowledge focus would need to be relevant - grounded in my research setting, supported by the data, and ideally also valuable in its own right, worthwhile studying in terms of its potential to produce meaningful results. In order to establish a knowledge focus, however, I needed to tackle a more practical issue: How does a researcher elicit information about embedded knowledge from the very participants in whom it is embedded? I realised that the embeddedness, or stickiness (Szulanski, 2000), of embedded knowledge was a potential barrier to my research! These issues were in fact deeply intertwined: In order to establish a knowledge focus, I needed meaningful data to engage with, and in order to elicit quality data from participants I needed good interviews tactics. This in turn required me to have some understanding of the nature of the knowledge involved in the research setting, and particularly of what was most meaningful in this setting, the knowledge focus. The reciprocal relationship between these issues is shown in figure 9, along with the interpretive toolkit that I developed to help manage them. This metaphorical toolkit comprised a set of iteratively developed interview tactics, a knowledge focus and a working mental model of the dimensions of knowledge.

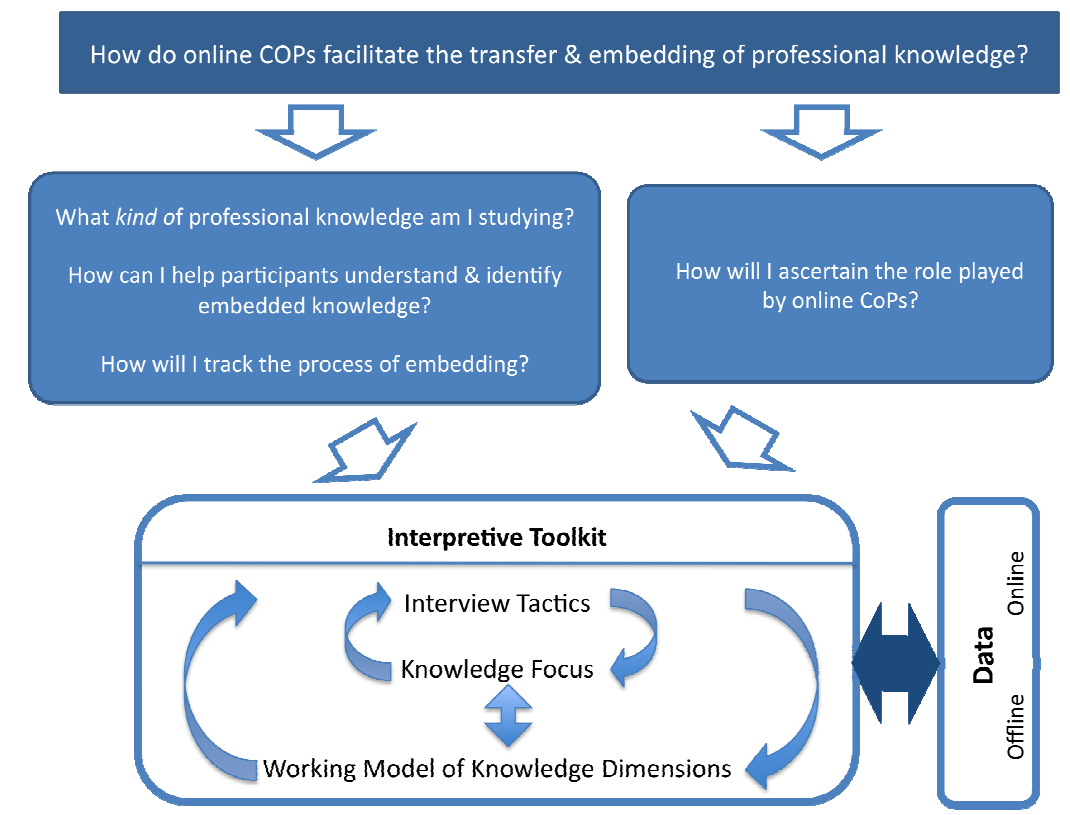

Figure 9: Key issues arising from my research question, and the reflexive development of my "interpretive toolkit" 
When I began interviewing, there was indeed some 'fuzziness' in my understanding. This resolved over time as my appreciation of the knowledge involved in the CoPs' KT activities grew, and as I built on this understanding, together with my experience of what was working in the interviews, to refine my interview tactics. This, together with my analysis of data, helped me to ascertain what knowledge was most meaningful in the study context - the new way. I began focusing in on this theme, leading to greater clarity.

I now return to the issue of how I determined a knowledge focus for the study. I begin with an examination of the wider governmental setting for my study, and an exposition of the high level agenda that was at play.

Having previously worked as a manager in government, I had an understanding of the MOE's emerging strategic direction for schools. In the early twenty-first century, educational policy was moving away from valuing the traditional subject-driven approach to learning, towards an approach favouring the development of a set of essential competencies in students, with the emphasis on developing each individual's potential. This resonated with contemporary knowledge management theory, valuing the ability for knowing above the delivery of knowledge as a product. Shifting the focus of schools to the fostering of essential competencies, defined as thinking, participating and contributing, self-management, relating to others, and using language, symbols and texts (MOE, 2006a), was seen as a means of improving equity of opportunity in education. From a social capital perspective, developing competencies was viewed as a means of producing the kind of flexible, innovative citizens who would help NZ flourish in a future that would be different from the twentieth century in ways we could not yet imagine; the so-called knowledge age (ibid).

This was a transformational agenda. At a high level, the government was promoting a whole new way of teaching and learning, as is made clear in the foreword to the eLearning Action Plan for Schools 2006-10 by the Minister of Education:

The answer lies in reorienting the system. Our challenge, as we examine how to make a difference (that is, how to ensure equal opportunity in education) is to reorient our system away from the organisation to the learner... Placing the learner at the centre of the education system is [a] radical notion... (Ministry of Education, 2006a, p.3).

How would this reorientation come about? Placing the learner at the centre would have profound change implications for both teachers and schools, but top-down 
approaches to educational change have a history of failure (Hargreaves, 2003) and the MOE had no managerial authority over schools. The MOE's approach to change, in the context of its ICT-based initiatives, is outlined in its eLearning Action Plan for Schools 2006-10 (MOE,2006a):

The focus on the use of ICT in schools has been on encouraging and monitoring change by teachers in their classrooms, that is, a bottom-up model with support and guidance from the centre. It is an approach that encourages reflective practice and collaboration within schools and between clusters and across wider communities of practice..."

(MOE,2006a, p.7)

The ICT PD programme sat within this Action Plan and operated via a partnership model, as outlined by the programme leader:

"[We\}... determine the viability of the [proposed] programme, and [the ability of] that group of schools to implement it, and then work in partnership with them through funding. Each and every one of those individual clusters can have a unique set of goals... It's based on the premise that the schools know best what suits them, in terms of the professional development of teachers." (Programme Leader, interview)

This is an example of the normative-re-educative approach to effecting change in human systems (Chin \& Benne, 1969). Unlike the power-coercive approach (which uses power structures to elicit change) and the empirical-rational approach (which emphasises the linear dissemination of an externally determined approach), normativere-educative change strategies involve the individuals themselves in determining the nature of change.

Initially, I failed to see a strong correlation between the ICT PD programme and this higher-level change agenda. As outlined earlier, the programme had a somewhat diffuse set of official goals, which had been developed some years prior to formalisation of the student-centred approach. A website described the programme in 'safe' terms as a "self-designed programme that promotes increased understanding by teachers, principals, students and school communities of the educational benefits of ICT" (MOE, 2008). However, by the time of my study, the programme was into its ninth year, and over half of NZ schools had participated in it. Two critical data sources provided evidence that by now, the larger educational change agenda was underpinning the programme.

A Powerpoint presentation, provided by the programme leader, was the first of these sources. A slide outlining the programme's three-year structure (see Appendix D) was provocatively entitled "Programme for Transformative Change". It positioned the 
programme as being "grassroots driven around MOE strategy" and used the key phrases "readiness for change" and "learner-centeredness". The second piece of indisputable evidence appeared in a statement about the ICT PD programme by the programme provider. This statement made explicit the fact that, at least in terms of "the view from the top", the change agenda was at the heart of the programme:

We are not [simply] learning how to use ICT, nor are we involved in developing ICT resources to match our curriculum. We are working on a new pedagogy for learning - looking at a new way of teaching. (Ham, cited by Cognition, 2007)

These pivotal data sources confirmed that, in reality, the ICT PD programme had a very ambitious intent. Its underlying goal was to spearhead transformative professional change, to instil the "new way of teaching" in participating schools, using ICT as a lever, or perhaps a Trojan horse. This change was to be driven by on-the-ground change agents (lead teachers). It required the buy-in and support of leaders and colleagues:

When teachers are changing, they start to make demands on the school system - it might appear as demands for new equipment, but it's really to do with the new pedagogy...When a teacher recognises the new paradigm and they're ready to lead the way, they hit so many barriers Unless they're supported by good leadership, and can find others who are doing the same thing, and there's a supportive culture, they will fail. (Project Leader, interview)

My finding about the 'true intent' of the programme occurred partway through my data gathering process and was critical. It positioned the new way as a candidate knowledge focus for my study. Given the grassroots nature of the ICT PD programme, there were two potential approaches that I could take in this study. The first was for me to investigate the transfer and embedding of all and any kind of professional knowledge that was involved, treating all knowledge as equally relevant. The second approach was for me to focus more exclusively on the embedding of knowledge concerning the new way. This was my preferred option.

Embedding the new way required teachers not only to incorporate new methods and technologies into their practice, but also to reframe their understanding of their profession and its source of value. If participating schools and teachers bought into this goal, they would need to relinquish their traditional role as a leader of learning and instead become a facilitator of individual, student-led learning, focusing on the fostering of skills for knowing rather than on knowledge as a product. Adopting the new way as 
a knowledge focus would therefore position my study as being about deeper, transformative professional change within the normative-re-educative tradition. I saw this as being a highly worthwhile, relevant approach for my research. Professional change is an issue of widespread, cross-sector concern. (The need for IS research to be of real-world relevance is such a recurring theme in the literature that is difficult to imagine a $\mathrm{PhD}$ candidate who does not have this engraved into their psyche!)

An additional source of relevance lies in he fact that in normative-re-educative programmes, dialogue amongst programme participants is seen as critical to their success. This is because normative change "entails understanding one's beliefs and knowledge and determining whether or not to change them" (Richardson \& Placier, 2001). My research was focused on the role of online CoPs, which provide opportunities for dialogue.

Having established the new way as a candidate knowledge focus, I needed to confirm its suitability. How well did the view from above, of those who might be called the paradigm pushers, match the view of received professional knowledge as seen from the ground, by the participants in my study? Knowledge about the new way would need to be sufficiently grounded in my research data to confirm that this was indeed the essence of what was being embedded. In order to ascertain this, I conducted a matching exercise, initially at an intuitive level and later more formally, exploring the fit between the themes emerging from the interviews and online data (bottom-up themes) and the change agenda (top-down themes). I analysed information about embedded professional knowledge, establishing a set of themes within an umbrella category called "nature of (professional knowledge) change".

Figure 10 shows the aggregated themes that emerged from this bottom-up analysis (bottom row), and compares it with a list of top-down themes that I established by analysing information associated with the ICT PD programme, including the eLearning Action Plan, MOE presentations, and my interview with the programme leader (top row). In order to clarify the extent of fit between top-down and bottom-up views, I grouped these two sets of change-related themes into three groups: (a) common (core) matched themes concerning the new way, (b) contextual (non-matched) themes to do with the new way, and (c) other change-related themes which were not closely connected with the new way. I found that there was a strong core category of matched themes. 


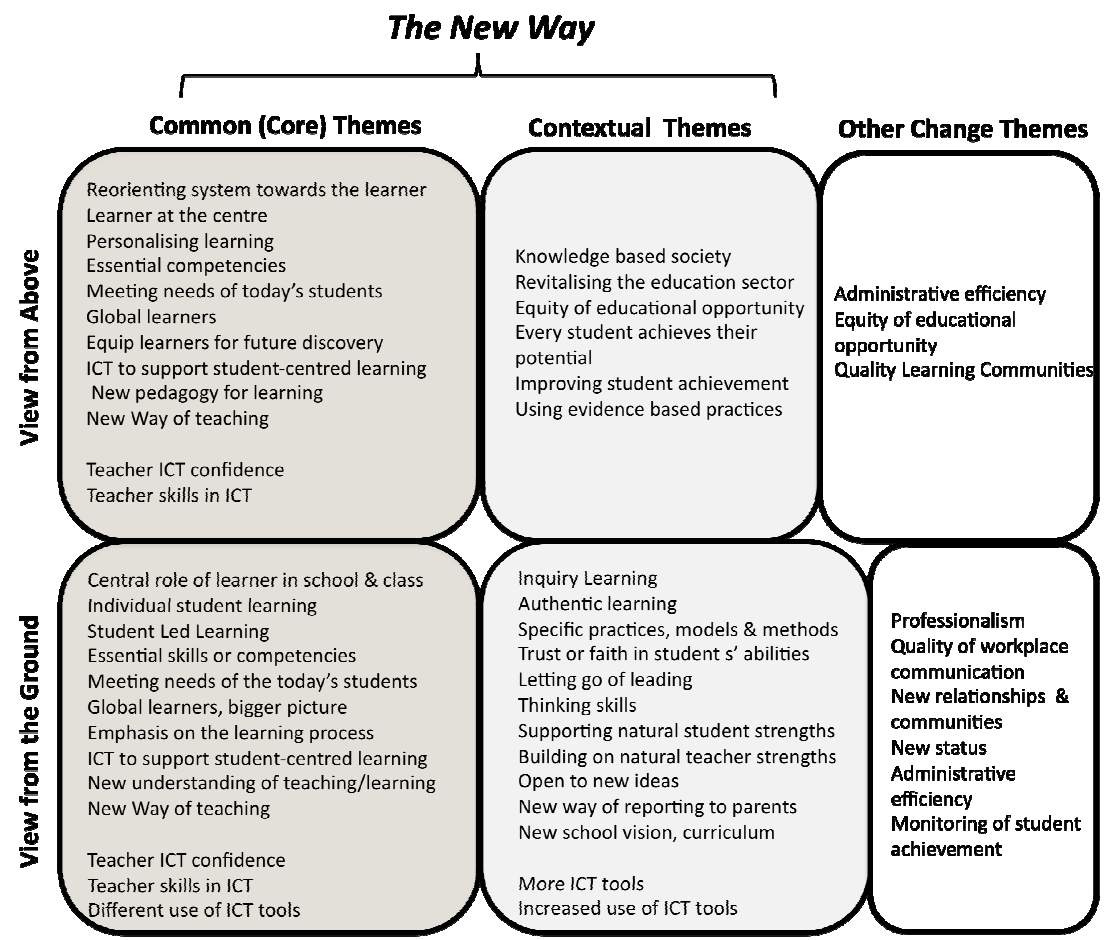

Figure 10: Establishing a Knowledge Focus: Comparison of top-down \& bottom-up themes

The themes in this core (left hand) category were well represented in the data from all but one of the five participating communities; CoP D. Their relative absence in this CoP can be explained by the fact that this CoP had completed the programme over a year prior to the others involved in this study. I had expected its maturity as a cluster to provide me with useful data about knowledge embedding, but this assumption proved erroneous. A Ministry official had described managing the programme as being "like working in shifting sand". I realised that because CoP D belonged to a different cohort from the others, it had not been exposed to the programme's recent focus on the new way. Instead, it had based its involvement in the programme around introducing a new technology for distance teaching. Perhaps co-incidentally, this cluster's online community had entered a period of stagnation, providing me with little useful data, so it was relatively easy to make the decision to prioritise my knowledge focus, and view this CoP as an unproductive sub-case.

A further source substantiates the relevance of the new way as a knowledge focus. This is a quantitative evaluation of national trends in ICT PD programme, focusing on the outcomes reported by the 2004-6 cohort (Ham, 2008). This study found that: 
"The (ICTPD) programmes were seen by the majority of participants as having been a 'significant' event in their overall development as teachers, which for many contributed well beyond any ICT-specific issues of increased technical skill, to encompass improved understandings in relation to teaching and learning more generally. Some $74 \%$ of the teachers indicated that the programme had contributed new ideas about teaching and learning, including $12 \%$ who felt that the programme had provided them with a whole new approach to teaching and learning" (ibid, p2).

The report goes on to articulate the nature of these new ideas, which include learnercenteredness. The findings from this study can be seen as confirming the relevance of my knowledge focus to the wider programme. Because I had been pointed towards clusters where the Ministry saw the programme as having been successful, and because the majority of my participants were in positions of leadership - lead teachers, informal leaders and facilitators, it is likely that I had strong representation from within the latter 12 percent. My data certainly suggested this.

Finally, in order to further clarify the knowledge focus that had emerged, I focused in on the category of common themes. I identified four recurrent key subthemes of the new way, as represented in both the top-down and bottom-up views. These themes were: (1) student-centred learning, (2) the teacher's (new) role as a learning facilitator, (3) a focus on the learning process and associated skills (or competencies), and (4) the integration of technology to support student learning. I then mapped the data from each community onto each subtheme, to ensure there was fit at this level of granularity. I found that there was an excellent fit with each of the key themes for CoPs A,B, C and E. Figure 11 shows the four key subthemes of the new way. (Appendix $E$ provides examples of these themes from each community.)

In summary, the exercise outlined above confirmed the thematic essence of what professional knowledge was being embedded, and what mattered most, in the study context. It clearly positioned my study in terms of its wider relevance to transformational professional change, and provided me with a means of focusing my interview questions and interpreting the data. In this respect, my knowledge focus can be seen as interpretive tool. 


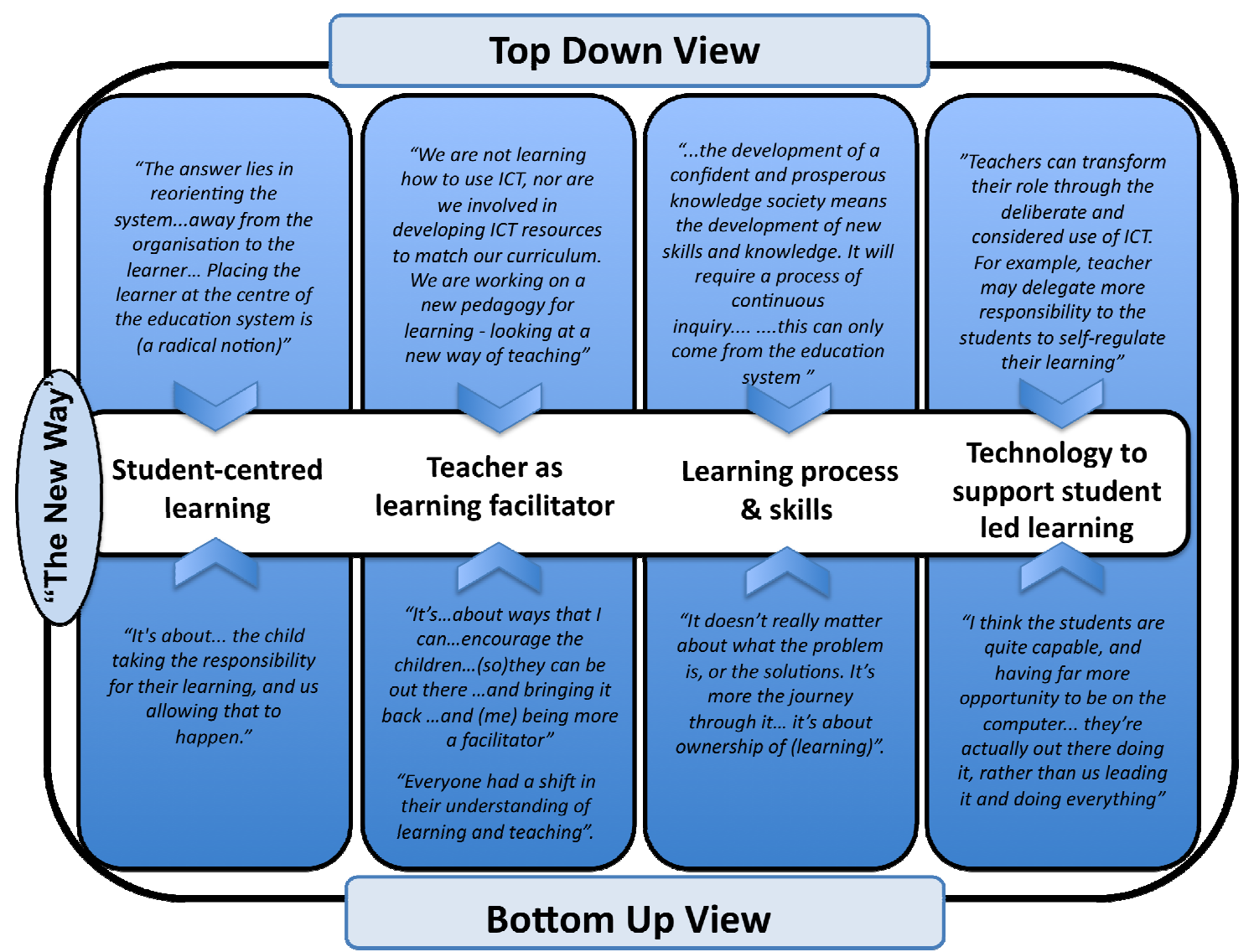

Figure 11: Establishing a knowledge focus: the new way - four key themes

Once I had established the new way as the focus, my understanding of the professional knowledge involved improved, and I was able to my interviews more exclusively and more meaningfully around the central how question. Figure 12 illustrates how I honed in from an early broad "what and how' approach in my questioning, to a more focused "how" approach once the knowledge focus was clear.

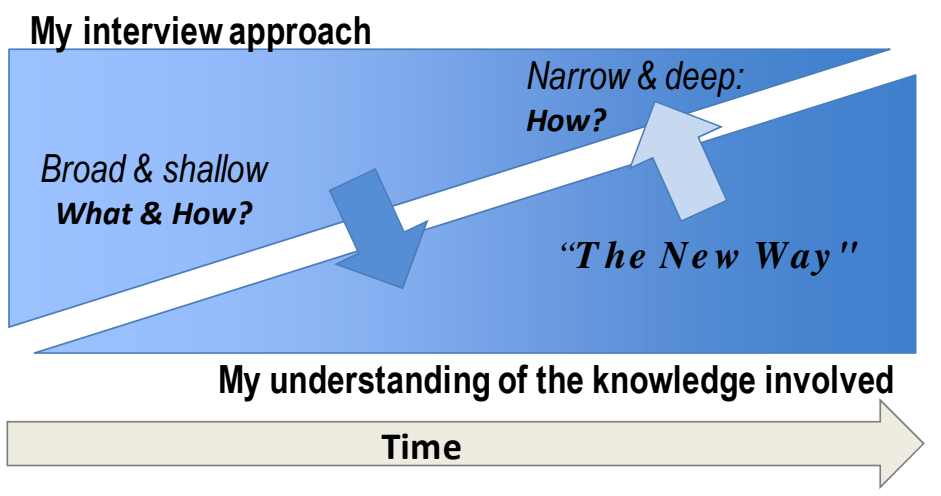

Figure 12: How clarification of a knowledge focus helped me to address the how question 


\subsection{Eliciting information about embedded knowledge}

As I contemplated how to identify the knowledge that was being embedded in my research setting, I realised that I faced a very practical challenge: How would I elicit information about embedded professional knowledge in my interviews? Given the degree of abstraction inherent in the notion of embedded knowledge, how could I make the concept relevant to a group of practically-oriented school educators? Most importantly, what would it take for these people to be able to recognise the professional knowledge that was embedded in their own, unique contexts? There was a paradox in the fact that I would be asking people in whom knowledge had become embedded (or was becoming embedded) to isolate and articulate it; to effectively disembed this knowledge. If embedded knowledge was as sticky as Szulanski suggests, would this be like trying to extract the egg from a baked cake?

My response to this issue was a decision to manage the interview process as effectively as possible to elicit the kind of information I needed. I worked on developing a set of interview tactics that would help address the challenge outlined above. Prior to entering the field, I revisited my draft interview script, drawing on the literature that I had reviewed, and infusing the script with a range of synonyms for embedded knowledge and knowledge embedding. I aimed for a mixture of jargon-free everyday terms and explanatory phrases that people might connect with, and which might help them to understand the concept of embedded knowledge and what it meant to them. My preliminary interview script, for example had included the statement:

I'm interested in understanding how professional knowledge is transferred; in particular, I'm looking in to how online communities help to transfer new teaching knowledge and new ways of working, so that the new ideas are taken up by people and/or schools...

Once I had incorporated new terms, the statement read:

... so that these new teaching ideas and ways of working are taken up more deeply by people or schools - to the point where they 'stick' or become embedded; become part of everyday routines...

While this was an improvement, I soon discovered that mentioning embedded knowledge in the same breath as online communities was overwhelming for participants. I therefore changed the structure of my interview. After introducing my research topic, I spent the first part of the interview encouraging people to describe what knowledge had been embedded and to talk broadly about how this had occurred. 
In the second part of the interview, I visited the how question in more detail, focusing exclusively on the role played by online communities. (I discuss issues relating to online CoPs below.)

When asking about what knowledge had become embedded, I achieved the best results by asking people what had changed in their teaching, ideas and understandings as a result of the ICT PD programme. Replacing the abstract term knowledge with these kinds of phrases appeared to make it easier for participants to recall such changes, and avoided the restrictions that might arise from individual interpretations of what did or did not constitute knowledge. In order to elicit examples of embedded knowledge, I asked all participants to identify a specific way in which their approach now differed from that at the start of the PD programme:

Please think of an example of a new teaching idea, approach or method that you've picked up during the ICT PD programme and that you now use regularly; something you're doing differently from before. What is this?

Once they had established a specific element of (new) teaching knowledge to focus on, I would return later in the interview, and explore what part online communities had played in helping to establish, or embed, this idea.

On the whole, this approach proved successful. Participants readily recounted a range of changes, including changes to teaching practice, their use of technology, their understanding of teaching and learning, their beliefs about education, their attitudes towards students, and their use of specific learning models and methods. Embedded knowledge did, nonetheless, prove to be sticky. Two participants had particular difficulty in recalling the nature of change. One of these people was heavily pregnant and had difficulty recalling any details of the programme. (I saw this as a temporary physiological issue!) The other person, a lead teacher, seemed to be unable to access and/or articulate what had been embedded:

I don't want to think about it too deeply now..(but) it's all there.. It's waiting to be called on....I don't remember all the specifics. I remember what I need to remember at the time, and if I want to go back to it then l've got those (online) readings. (Lead teacher, interview)

This comment was reminiscent of Davidson and Voss's (2002) description of knowledge as being so deeply embedded that workers "no longer think about what they're doing but simply do it" (p.63). The well-known concept of unconscious 
competence also came to mind. I interpreted the difficulty that this teacher reported as arising from the embeddedness of the knowledge under investigation. However, I also noted in my research log: "Unconscious competence" presents a challenge for the researcher who is 'looking for' embedding as it is not possible to determine whether there is genuine embedding or belief". This interview was perhaps most useful in that it reminded me that the information I was eliciting from participants was, at best, only part of the picture in terms of the professional knowledge that had become, and/or was becoming, embedded in my participants.

Questions about knowledge that had stuck were often successful in eliciting recognition from interviewees; for example:

What is it that makes a new idea stick so it becomes more deeply embedded in what you do and how you think?

The term stuck also arose spontaneously:

Some of our ideas have definitely stuck, and stuck very well. (Principal, interview)

Others spoke of knowledge gradually sinking or soaking in:

It's like, you're finding out information on-line, and you try three different sources.... It takes a while to sort of soak in.... For me it might take a couple of years to become soaked in. (Teacher, interview)

As I gained more interviewing experience, I found that different people responded differently to different phrases and my interview tactics evolved to become more improvisatory. If a particular descriptive phrase resonated with a participant, or if a participant used their own terminology, I would reiterate these terms within the course of the interview. My improvisation was guided by building on whatever language worked best for each participant. After testing out a few analogues for embedded, such as "stuck" and "stayed with you", I repeated the term(s) they responded best to.

Earlier in this chapter I identified a knowledge focus and a set of four key sub-themes. As the new way emerged as the knowledge focus of my study, I saw it as inappropriate to prompt interviewees about any particular themes or categories of knowledge. Instead, I cast the net widely, asking people what had changed in their practice and understanding. As much as possible, I treated professional knowledge as a package, leaving it up to individuals to elect their own focus. My growing awareness of topical sub-themes did, however, help me recognise where to 'dig deeper' in the interviews. 
My second way of identifying embedded knowledge was through online community records. Themes and ideas that were becoming embedded were easier to recognise and track in written form, for example, in forum and blog postings where people were outlining their opinions and ideas, and/or describing and critiquing their practice. Once I had established the new way as my focus, I was able to identify relevant recurring themes and metaphors. I could then review participants' recycling of these phrases in relationship to the theme of embedding - the reuse (appropriation, adaptation or personalisation) of key themes helped to make the process of embedding visible.

\subsection{Probing into the role of online CoPs}

I faced a further challenge - how to ascertain the actual role of online CoPs in the embedding of professional knowledge. There were two aspects to this problem. Firstly, members of school clusters participated in both face-to-face and online interactions. This made it difficult for them to ascertain which, if any, aspects of the professional change process could be attributed to the online dimension of these CoPs. (This was not an issue for members of CoP E, a highly distributed community.)

Secondly, the term online community means different things to different people. As has been outlined in the literature review, online CoPs have undergone relatively recent change, as formal facilitated, platform-centric communities have been supplemented and/or replaced by non-facilitated, open, multi-platform online community structures. In NZ's school sector, the term online community has been historically associated with web-based resource centres. I found that when I asked teachers about their participation in online communities, they readily recounted their use (or non-use) of these resource centres, and their participation in official online forums. However, they were less likely to mention their involvement in nameless, non-official, personal communities, such as their interactions with colleagues via IM tools (iChat and Skype), their email communications with a blogging colleague, or even their own blogging efforts.

Once again, I relied on a combination of interview tactics and online data sources to help me address these issues. I developed my line of questioning about online CoPs to follow a broad three-part structure - opening up, probing and rounding up. I began by opening up the concept of online communities. I asked participants what online communities they participated in and what role they played then explained that I was interested in the full range of their online involvement with colleagues via online 
technologies. I often gave a range of examples (e.g. online forums, instant messaging, blogs) to widen the net and help people expand their thinking. Sometimes I prefaced the phrases online CoP or online community with more generic expressions, such as online professional relationships:

I want to talk to you about the on-line professional relationships that you have; how that has contributed to the journey, the change that you've experienced. How would you describe the on-line communities that you belong to?

After this, I moved into a sequence of probing questions, aiming to draw out stories about the nature and influence of participants' online interactions, while addressing my research sub-questions about the influence of different technologies and roles. These probing questions were tailored to each person's situation. They took into account the nature of professional changes they had reported, their role, and their varying levels of cognition about the professional impact of their online engagement with CoPs. Table 6 illustrates the range of probing questions I used.

Some participants had produced an online trail of CoP engagement through their participation in blogs, social bookmarking sites and/or online forums. This data provided me with a series of snapshots of change as it had occurred over time. It also gave me a preview of teachers' interests, tool use, methods and relationships, which I would read prior to the relevant interview, then reference during the interview, allowing me to focus on relevant issues and probe more deeply.

Through probing, I discovered that there was a pervasive invisible dimension to many people's online CoP interactions. Some teachers operated almost exclusively in online contexts where they left no accessible record, such as IM (Skype and iChat), Twitter, and on the periphery of Web 2.0 communities, via 'behind-the-scenes' emails. Individuals with a strong visible online presence also undertook invisible communication, counterpointing this with more visible forms of online engagement. There were few online records available for me to 'see' these invisible interactions, so I was strongly dependent on interview tactics to help me understand the function of these invisible spaces. I asked participants to explain the reasons for their choice of online communication tools, and about how and why they used particular tools.

In the interest of ascertaining participants' own views of what was (and was not) an online CoP, I made a point of asking each person how they viewed the group of people with whom they interacted online - was this a community, or was it more of a network? 
Although participants gave varying descriptions of the online communities they belonged to, they nonetheless all stated that they felt the term online community was an appropriate descriptor.

\begin{tabular}{l|l}
\hline $\begin{array}{l}\text { Description } \\
\begin{array}{l}\text { Questions about the influence } \\
\text { of online CoPs }\end{array}\end{array}$ & How does that community work for you? \\
\hline $\begin{array}{l}\text { Questions about specific } \\
\text { technologies }\end{array}$ & How do you decide what communication technologies to use? \\
\hline $\begin{array}{l}\text { Questions about roles \& } \\
\text { relationships }\end{array}$ & $\begin{array}{l}\text { I'm interested in (understanding) the... skype and i-chat conversation that goes on. What's } \\
\text { your use of those kind of tools in the ICT community? }\end{array}$ \\
\hline $\begin{array}{l}\text { (Through) your blogging, and your Delicious...what kind of relationships have you developed...? } \\
\text { embedding process }\end{array}$ & $\begin{array}{l}\text { Who are the influential people in the communities that you belong to? How are they } \\
\text { influential? Who has been influential in terms of new ideas? }\end{array}$ \\
\hline $\begin{array}{l}\text { At what point does an idea move from something that's being quoted (in your blog) to being } \\
\text { your own idea? What happens over that time? }\end{array}$ \\
\hline $\begin{array}{l}\text { Questions building on online } \\
\text { data records }\end{array}$ & $\begin{array}{l}\text { At what point does an idea move from something that's being quoted (in your blog) to being } \\
\text { your own idea? What happens over that time? }\end{array}$ \\
\hline I'm interested in the online relationship you have with (Susan)...How has (it) helped you with \\
(establishing that) practice?
\end{tabular}

Table 6: Examples of probing questions about the role of online CoPs in embedding knowledge

This question produced useful reflections on the fluid, informal nature of CoP E, a nontraditional, blog-centric community:

(People might think that) the online community I belong to is somehow structured... but [it's] very informal and fluid. I am of the opinion...that [this] works much better. Formal and fixed communities such as [those used] for educational purposes tend not to have such a free flow of ideas.... People are scared of being wrong in those contexts and appearing stupid or ill informed, whereas this community that I seem to belong to there is an easy nature about it.... (Teacher, e-mail)

Finally, I asked one or two rounding up questions, bringing my central research question into the spotlight. I asked participants to reflect back on the online experiences they had recounted and to consider whether their online community involvement had contributed in any way to the embedding of knowledge, helping new ideas and methods to 'stick' - and if so, how had this occurred. An answer to the how question is not at all easy to arrive at (it is the subject of this thesis!) but my rounding up questions elicited a range of valuable personal insights. 
As the following example illustrates, they also highlighted where participants saw online $\mathrm{CoP}$ interactions as having played an important role in professional change:

....in terms of our inquiry focus, and incorporating thinking skills into what we do - that's a direct result of our online community work. (Lead teacher, interview)

In combination, the interview tactics outlined above, together with online data, provided me with relevant and useful data about the role played by online CoPs in relationship to the embedding of professional knowledge. As I analysed this data and my preliminary findings began to emerge, I was able to use later interviews to dig even deeper into how online CoPs and their associated technologies, roles, and relationships contributed to the phenomenon of embedding.

\subsection{The dimensions of professional knowledge}

As I analysed interviews, honing in on the thematic nature of the knowledge which was being embedded, I discovered that I was not only looking at a set of knowledge themes, but also at a number of dimensions of professional knowledge. Knowledge to do with the new way included people's theories about the new student-centric paradigm and the role of technology, their supporting beliefs and values, their personal interpretive frameworks (how they described, understood and critiqued their teaching and their students' learning, applying theories and metaphors), various learning models, action-based knowledge to do with practical methods and the use of technologies, and even school vision statements.

As has been outlined in Chapter 2, the literature about professional knowledge presents diverse and competing conceptualisations of what constitutes professional (teachers') knowledge and how it is structured. While some researchers have focused on identifying categories of teacher knowledge, others see this as counterproductive.

Borko and Putnam (1996), for example, note that:

A potential danger inherent in any description of categories of knowledge is that people may come to see the categories as representing an actual storage system in the human mind rather than a heuristic device for helping us think about teacher knowledge. That is, we may find ourselves thinking that teacher knowledge is organized into abstract, isolated, discrete categories, whereas, in fact, what teachers know and believe is completely intertwined, both among domains and within actions and context (p.677).

I, too, saw the reality of professional knowledge as a fluid, multi-faceted amalgam, with my data making it clear that beliefs were inseparable from knowledge: 
I think it becomes the embedded belief that you have yourself about something, so it adds to you. (Teacher, interview)

Despite this, I felt that it was useful to investigate the dimensions of the professional knowledge involved in my study context. Based around my emerging analytical codes, I drew a series of exploratory diagrams, trying to conceptualise the nature of the professional knowledge in my study. Figure 13 provides an early example, spanning both content categories (students, methods, identity, technology) and higher-level categories (theories, metaphors, beliefs).

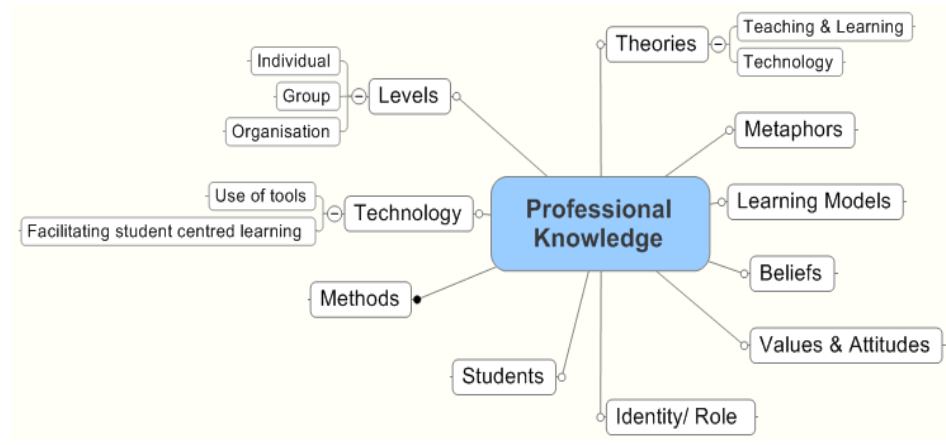

Figure 13: Early exploratory map of professional knowledge (based on NVivo codes)

These were rough working models that changed continuously, reflecting the fluidity of my mental models. The challenge was such that I did not produce a 'final' version, but the exercise, and my continual refection on the make-up of knowledge made me sensitive me to the existence of its composite dimensions.

I found that my data provided many rich examples of beliefs, values, theories, practices and models, and - significantly - several standout examples of movements between these dimensions. Following my visit to a cluster that made strong use of online forums (CoP A), I established a data category called "theory-practice link" and gained a sense that the process of moving between the theoretical and practical dimensions of knowledge was important to the embedding process:

For me it was looking at the theory of teaching, and the practice. Both those aspects came through. And the theory backed up what you were doing practically, and if you were doing something practically you could often match it up with something in the theory, and so, "That's why we do that."

[I]: If you pick up an idea and try it, what makes you stick with it?

I guess if it's working or not. And I still have to feed myself with going back and reading again... and going back refreshing the whole thing what it's about, and refining it... (Teacher, interview) 
My inability to settle on a single model that 'worked' led me to examine the literature surrounding the development of teachers' knowledge in more depth. I found two metareviews of research into teacher knowledge and teacher change that contained material that strongly resonated with my emerging model. According to Munby et al. (2001), research into teachers' knowledge is characterised by a dichotomy between theoretical knowledge, based in the paradigmatic mode of thought, and practical knowledge, based in the narrative mode. These can also be seen as reciprocal dimensions of knowledge, or as a duality. While theoretical knowledge is typically declarative, abstract and conceptual; practical knowledge is more procedural, specific and pragmatic (Leinhardt et al., 1995, pp. 402-403).

Of greater relevance, I found that Leinhardt et al (ibid) view the development of teachers' knowledge as a recursive process based around the integration of these dualities. The level of integration needed for effective practice does not result from the simple 'translation 'of theory into practice, but instead requires a reciprocal process of transformation of each kind of knowledge into forms associated with the other. They see individuals as needing to continually particularise theories and abstract theories from practice (p.403), making iterative, reciprocal readjustments. This theory gelled strongly with statements made by several participants, and validated the importance of my "theory-practice link" category.

In later iterations of my working model of knowledge, I elevated the paradigmatic/practical knowledge divide, grouped beliefs, theories and values together into the category paradigmatic knowledge, and identified more granular categories within each dimension, based on deeper data analysis. (See figure 14).

Another aspect of Leinhardt et al.'s paper gelled for me. This was the authors' comment that traditional workplaces provide barriers to the integration of paradigmatic and practical knowledge, notably through a lack of time for reflective and analytical thought. The ICT PD programme leader had portrayed teachers as being "time poor" and teachers' lack of time for deeper thinking had been a recurring theme in interviews. In 1995, a year after the first W3 consortium meeting, Leinhardt et al. had called for universities to create "structures to slow down time" so as to facilitate reflective revision and knowledge transformation (ibid, p.404). I wondered whether online communities might be helping to achieve this. 


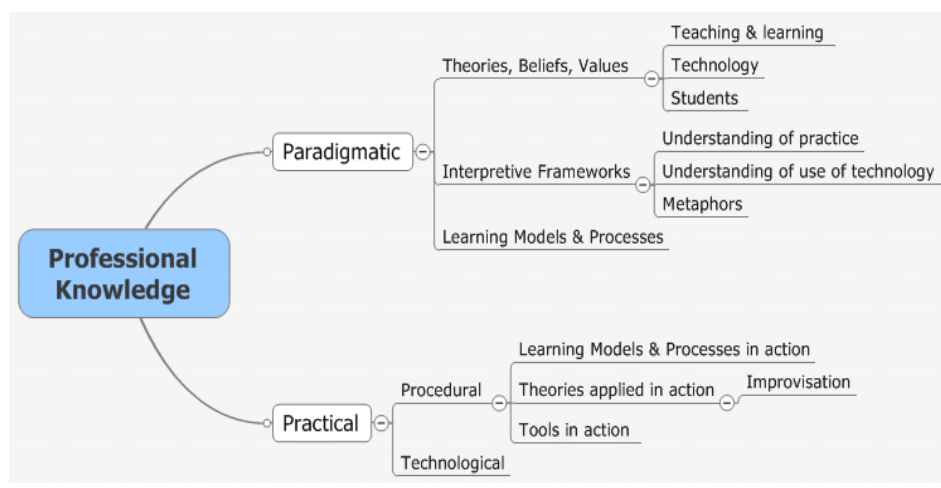

Figure 14: Later working model of knowledge dimensions

As noted above, my mental model of the dimensions of the professional knowledge being embedded was very fluid. It was nonetheless a valuable interpretive tool, and the drawing of diagrams based around emerging codes proved to be a useful practice. It helped to sensitise me to important aspects of my data and the difficulty of the exercise provided me with a strong incentive to dig more deeply into the relevant research literature, linking this back to my findings.

\subsection{Chapter summary}

In this chapter I have used the metaphor of an interpretive toolkit to show how I used a series of provisional models and an evolving set of interview tactics to mitigate the potentially fuzzy nature of my research question. Developing this toolkit provided me with a clear knowledge focus for my study: the embedding of the new way, or paradigm of student-centred, technology enabled teaching and learning. It also provided me with tactics to helped people access relevant stories about embedded professional knowledge, and to cast the net sufficiently widely when investigating people's participation in online CoPs.

I have demonstrated the relevance of the new way as the knowledge focus by a topdown/bottom-up matching exercise, showing how four key sub-themes appear prominently in the data from the five of the CoPs in my study. (I have also explained the reason for their non-appearance in CoP D.) In addition, I have argued that my knowledge focus elevates this study's relevance to practitioner communities, positioning the study as being about the embedding of knowledge in the context of transformative professional change. 


\section{Overview of research results}

\subsection{Introduction}

This chapter provides a background to my research results and presents a brief overview of the key theoretical findings.

In the first section, I outline my findings about the five Communities of Practice (CoPs) in this study. Firstly I present an overview of each CoP and the extent to which it relied on online means to facilitate the embedding of the new way. I identify key contextual issues that may account for differences between the cluster-based CoPs. Following this, I discuss the significance of my discovery of CoP E. Not only did this community challenge my conceptions about the nature of online CoPs, but my decision to include it in this study led me to take a new perspective on the case: Rather than viewing it as a set of parallel subunits, I developed a more complex, system-based awareness of how online CoPs were facilitating knowledge transfer (KT) and embedding in the research setting. I conclude this section by discussing the structural relationship between the five communities, showing how CoP E acted as a non-official but important connecting community.

In the second section of this chapter, I summarise my research results at a higher level of abstraction. The principal outcome of my research project is a three-level explanatory theory (Gregor, 2006). It comprises three complementary mechanisms for facilitating knowledge embedding, operating at the micro, meso and macro levels. I provide a brief introduction to each mechanism in this chapter; outlining them in detail in Chapters 6-8. In addition to this three-level theory, I identified five common underlying processes that facilitated the embedding of knowledge of the new way in the research case. In the final part of this chapter I introduce each process and discuss how it promoted the embedding of knowledge. 


\subsubsection{Findings about the five CoPs}

\subsubsection{The ICT PD cluster CoPs}

ICT PD clusters were self-selected groups of schools that determined their own programme objectives. Their online communities were part of blended CoPs whose members engaged in both online and offline communication. Face-to-face communication occurred more frequently within cluster schools than between them, but this was at lower level than in many work settings. (Teaching is a solitary profession, with most time being spent alone with students. In NZ this "student contact time" exceeds the OECD average (Statistics NZ,2008 ).) Clusters ran several workshops a year, initially based around ICT basics, then focused on the effective use of ICT for teaching and learning. Schools used programme funding to give appointed lead teachers release time from classroom duties so they could support their colleagues, communicate with lead teachers at other schools, and undertake online activities. Cluster schools ran regular internal meetings, techie brekkies and workshops.

Each ICT PD cluster had an online community environment, hosted on a proprietary platform by the programme provider. This included programme information, online forums and a resource library, managed by the cluster facilitator and lead teachers. It also provided a resource bank with lesson ideas, technical advice, links to conferences, and administrative content, managed by a national facilitation team. At the time of my study, cross-cluster forums had been discontinued due to poor uptake, but membership of a national listserv was compulsory. Rather than being participatory, the listserv was used to broadcast programme information. Within clusters, schools managed their own resource areas, either within the official online community, or on another platform or shared drive. The 'official' online CoP infrastructure described above was used in different ways by different clusters, due to the programme's grassroots approach and the preferences of the schools involved. However, this official infrastructure was only the part of the online picture. Most clusters had also developed a non-official, informal online CoP infrastructure, to extend and complement the official one.

I now summarise my findings about the five participating CoPs. Communities $A, B, C$ and $\mathrm{D}$ were cluster-based CoPs with a strong official dimension. Community $E$ was an unofficial CoP with distributed membership and little face-to-face contact between members. (These findings are summarised, with the participant breakdown data, in table 7.) 
Table 7: Summary of findings from participating CoPs

\begin{tabular}{|c|c|c|c|c|c|c|}
\hline CoP & Description & Maturity & Research Participants (roles) & $\begin{array}{l}\text { Extent of embedding of the } \\
\text { new way of teaching }\end{array}$ & $\begin{array}{l}\text { Level \& nature of online } \\
\text { interaction }\end{array}$ & Communication media \\
\hline$A$ & $\begin{array}{l}\text { Cluster of primary schools } \\
\text { Formal - online \& offline }\end{array}$ & $\begin{array}{l}\text { Mature } \\
\text { Recently } \\
\text { completed } \\
\text { Year 3 }\end{array}$ & $\begin{array}{l}14 \text { participants at } 4 \text { of } 9 \text { schools } \\
1 \text { facilitator } \\
6 \text { lead teachers } \\
3 \text { teachers } \\
4 \text { principals/school managers }\end{array}$ & $\begin{array}{l}\text { High \& continuing } \\
\text { Whole-of-school change approach } \\
\text { Mutual support between schools }\end{array}$ & High; Mandatory & $\begin{array}{l}\text { Widespread/ significicant use: } \\
\text { Forum, Email, IM, SMS, Online repository } \\
\text { Face-to-face workshops \& discussions } \\
\text { Lesser use (amongst } 3+\text { members): } \\
\text { Listserv }\end{array}$ \\
\hline $\mathrm{B}$ & $\begin{array}{l}\text { Cluster of primary schools } \\
\text { Formal - online \& offline }\end{array}$ & $\begin{array}{l}\text { Mature } \\
\text { In Year } 3\end{array}$ & $\begin{array}{l}11 \text { participants at } 4 \text { of } 7 \text { schools } \\
1 \text { facilitator } \\
3 \text { lead teachers } \\
4 \text { teachers } \\
3 \text { principals/school managers }\end{array}$ & $\begin{array}{l}\text { High \& continuing } \\
\text { Whole-of-school change approach } \\
\text { Mutual support between schools }\end{array}$ & High; near-mandatory & $\begin{array}{l}\text { Widespread/ significant use: } \\
\text { Email, IM, SMS, Repository } \\
\text { Face-to-face workshops \& discussions } \\
\text { Lesser use (amongst 3+ members): } \\
\text { Forum, Listserv, Blog }\end{array}$ \\
\hline $\mathrm{C}$ & $\begin{array}{l}\text { Cluster of secondary schools } \\
\text { Formal - online \& offline }\end{array}$ & $\begin{array}{l}\text { Maturing } \\
\text { In Year } 3\end{array}$ & $\begin{array}{l}8 \text { participants at } 3 \text { of } 3 \text { schools } \\
2 \text { cluster facilitators } \\
2 \text { lead teachers } \\
1 \text { teacher } \\
3 \text { principals/school managers }\end{array}$ & $\begin{array}{l}\text { Underway } \\
\text { Opt-in teacher participation; school focus } \\
\text { Mutual support between schools }\end{array}$ & Low; opt in & $\begin{array}{l}\text { Widespread/ significant use: } \\
\text { Email, Video-Conference, IM, SMS, Online repository } \\
\text { Lesser use (amongst 3+ members): } \\
\text { Forum, Listserv }\end{array}$ \\
\hline $\mathrm{D}$ & $\begin{array}{l}\text { Cluster of secondary schools (\& two } \\
\text { composite schools) } \\
\text { Formal - offline }\end{array}$ & $\begin{array}{l}\text { Inactive } \\
2+\text { years } \\
\text { post year } 3\end{array}$ & $\begin{array}{l}9 \text { participants from } 4 \text { of } 8 \text { schools } \\
0 \text { facilitators } \\
1 \text { (ex) lead teacher } \\
3 \text { teachers } \\
1 \text { technical co-ordinator } \\
4 \text { principals/school managers }\end{array}$ & $\begin{array}{l}\text { Negligible - focus on tool use \& technology- } \\
\text { led modifications to practice }\end{array}$ & Inactive as online CoP & $\mathrm{N} / \mathrm{A}$ \\
\hline $\mathrm{E}$ & $\begin{array}{l}\text { Virtual 'Web 2.0' community } \\
\text { ('community of passion' for ICT use) } \\
\text { Informal - online (virtual) } \\
\text { No official link to programme, but } \\
\text { overlapping membership with CoPs } \\
\text { A, B and C }\end{array}$ & Mature & $\begin{array}{l}17 \text { participants from } 9 \text { schools } \\
2 \text { also in Community A } \\
8 \text { also in Community B } \\
2 \text { also in Community C } \\
3 \text { also in other cluster CoPs } \\
2 \text { also national programme leaders } \\
\text { Roles in 'home' CoPs } \\
5 \text { facilitators } \\
4 \text { lead teachers } \\
5 \text { teachers } \\
2 \text { principals } \\
1 \text { other } \\
\text { Informal roles in this CoP } \\
8 \text { Connector-leaders } \\
9 \text { Follower-feeders }\end{array}$ & $\begin{array}{l}\text { Very high \& continuing } \\
\text { Distributed community focus }\end{array}$ & Extremely High & $\begin{array}{l}\text { Widespread/ significant use: } \\
\text { Email, Blog, RSS, Social bookmarking, IM, Twitter } \\
\text { Lesser use (amongst 3+ members): } \\
\text { Video-conference, Podcast/Teacher Tube, Second Life }\end{array}$ \\
\hline Other & Programme Leaders & & $\begin{array}{l}1 \text { Programme Leader (also in CoP E) } \\
1 \text { National Facilitator (also in CoP E) }\end{array}$ & & & \\
\hline
\end{tabular}




\subsubsection{CoP A and CoP B: Primary schools}

CoPs $A$ and $B$ were clusters of primary schools, catering for students in years 1-8 of their formal education. CoP A was in a predominantly rural region - two of the participating schools were based in small settlements and two in a nearby provincial centre. The schools in CoP B were located in rural townships. I interviewed 14 individuals across four of the nine schools in CoP A and 11 individuals across four of the seven schools in CoP B.

In both communities, knowledge about the new way had become well embedded at the cluster, school and individual level. Most participants recounted what they described as significant changes in their understandings about learning, the nature and content of teachers' informal workplace discussions, and their classroom practice. The four key subthemes of the new way, identified in Chapter 4, appeared regularly in interviews and online records associated with these CoPs (see examples in Appendix E). New school-level teaching approaches, and participants' buy-in to these, were signalled by the use of collectivist phrases, such as "Inquiry Learning is the way" (principal, CoP A) and "We're running with thinking skills" (teacher, CoP B). Inquiry learning and thinking skills are student-centred learning approaches associated with the new way. One or both of these approaches was in common use at most schools in these clusters.

At the time of my field interviews, new professional knowledge was still being formalised (routinised, codified and institutionalised) and differentiated (customised) by participating schools. CoP A's schools were at a more advanced stage of this process than those in CoP B, no doubt reflecting the fact that this community had recently completed the ICT PD programme - CoP B was part-way through the programme's final year. End-of-programme reporting requirements created an incentive to develop school-level results that would be seen as justifying the government's investment. This may have contributed to a ramping up effect in year three of the programme: CoP A experienced an upsurge in online forum activity in the third year, and a principal in CoP B spoke of being "in the thick of it".

CoPs $A$ and $B$ had both embarked on the ICT PD programme with a sense of focus and vision. This was centred, in each case, on the fostering of a student-led learning approach within schools, and facilitating students' use of ICT to support this.

Our school came into the cluster with a very clear vision; and that was inquiry learning...We believed our curriculum was broken, and we wanted to change it.... We spoke to [all the leaders and said] "Come on, 
here's ICT money, we want to change our approach. It's through ICT, but it's also through a change in pedagogy. (Principal, CoP B)

Both CoPs focused on the introduction of inquiry learning, while in CoP B there was an additional emphasis on developing students' thinking skills. These foci appear to have facilitated the embedding process - they were well articulated by participants, indicating a high level of common understanding and shared interpretive frameworks. Szulanski (2000) and Rolland et al. (2003) have found that shared cognitive bases increases absorptive capacity, facilitating knowledge transfer.

Most Interviewees from both CoPs A and B credited their online CoPs as having contributed to the embedding of knowledge and, in many cases, as having played a key role. This was stated most unequivocally by a lead teacher in CoP A, who said, In terms of our inquiry focus, and incorporating thinking skills into what we do; that's a direct result of our online community work.

In both clusters, online CoP participation had significantly increased teacher-to-teacher interaction and extended it outside of working hours. This was associated with the development of new relationships and the increased accessibility of colleagues' ideas, opinions, problems and work practices, which in turn lowered barriers to workplace engagement. As new ideas and challenges were introduced to each community through its participation in the ICT PD programme, online communications also played a critical role in allowing peers to benchmark their emerging thinking against each other. In both communities, the use of unofficial, informal and effectively invisible online communications (via IM tools) complemented the official, formal and more visible online infrastructure, leading to the establishment of a trusting, open, low-stakes communication culture.

CoPs $A$ and $B$ both made strong use of online communication tools, but had somewhat differing cultures of tool use. In CoP A, participation in online forums had initially been compulsory, resulting in a strong uptake of, and sustained emphasis on, this tool. CoP A's forums were used primarily to discuss professional readings and relate them to individual practice. CoP B also used online forums, but did so in a more episodic manner. Its forums were mainly focused around learnings arising from cross-cluster visits. (Those based on professional readings drew more limited engagement.) This aside, the members of both communities used online repositories of recommended teaching resources and received postings from a national listserv. In addition, 
members of both communities communicated regularly with each other via instant messaging (IM) tools, email and SMS (mobile text messaging). They also communicated face-to-face in workshops, meetings and discussions. The communication media used in CoPs A and B are itemised, together with other summary-level information, in table 7 . In both communities, taking into account both online and face-to-face communication settings, members can be seen as interacting within a set of diverse communication contexts, or professional engagement spaces. Each of these engagement spaces met particular professional needs and had its own conventions and culture of use. The significance of this diversity of engagement spaces in relationship to the embedding of knowledge is discussed in Chapter 7 . It forms the basis for my theory about the micro (individual) level knowledge embedding mechanism.

The accounts of participants from CoPs $\mathrm{A}$ and $\mathrm{B}$ about the knowledge embedding process indicated that a range of activities and issues had occurred over the two-tothree years prior to my visit. Data analysis showed that there were many commonalities in this process at the cluster and school level. The role played by online communities had changed over the course of the programme, as schools and teachers had moved from being challenged, through trialling new approaches, to eventually adapting and appropriating the new knowledge. (This finding - based on the analysis and synthesis of data from communities $A, B$ and $C-$ is the basis for my model of a six-stage meso-level mechanism for knowledge embedding, the knowledge embedding cycle.)

\subsubsection{Contextual issues: primary versus secondary CoPs}

The fact that CoPs A and B were clusters of primary schools appears to have been a significant facilitator of knowledge embedding at organisational level. In the primary years of schooling, teachers are responsible for teaching across the full curriculum, usually working with one cohort of students. This can be seen as creating ample common ground for professional practice initiatives, such as the ICT PD programme. In contrast, teachers at the secondary school level (years 9-13) teach one or two subjects - a narrow slice of the curriculum - to rotating groups of students. This results in a series of subject-based silos in secondary schools, and considerably less professional common ground. As one primary participant noted, there is a saying that primary teachers teach students, whereas secondary teachers teach subjects. This issue was noted by a number of participants, including the cluster facilitators and programme 
leader, as creating a problem for school-wide professional development initiatives ${ }^{4}$ at the secondary level. Lack of common ground is known to be a barrier to knowledge transfer (Rolland et al., 2003; Szulanski, 2000) as it results in low absorptive capacity a lack of shared cognitive bases, mental models and backgrounds.

CoPs C and D were clusters of secondary schools ${ }^{5}$. By including two secondary and two primary school communities in my research project, I had hoped to gain a better understanding of the contextual factors influencing knowledge embedding and the role of online communities in the research setting. Unfortunately, and in no small part due to the contextual issue noted above (primary versus secondary teaching), the data from these communities failed to match that of CoPs A and B in terms of its relevance, depth and richness. (The case of CoP D was particularly problematic, as I explain below.)

It appeared, in fact, that the ICT PD programme had more than one barrier at the secondary level. Firstly was the lack of common ground that made a whole-of-school approach difficult. This was evident in a less-than-clear sense of shared focus amongst CoP C's members, and a purely tool-based focus in CoP D. There was a second possible barrier in the larger size of the teaching pool in most secondary schools. Three out of seven secondary schools in this study had over 30 teacher full time equivalents (FTEs), teaching over 500 students. In these large schools, a smaller proportion of staff was engaged in the lead teacher role (a change agent role), making the task a larger job. The third, and perhaps most significant barrier, was the low perceived feasibility of whole-school change in secondary schools. Although participating principals saw shifting to a more student centred way of teaching as desirable, they did not feel such a major change could be accomplished within the time frame of the ICT PD project. The project was viewed instead as a means of kickstarting longer-term change:

What [secondary] teachers do... is pretty much the same as it was 30 years ago, and it's pretty much the same as it was 40 years ago.... when I started school. But I actually do think that in ten or fifteen years... it will finally, especially for the seniors, start to look considerably different... and it's this stuff [ICT] that will allow us to do things differently." (Principal, CoP C)

\footnotetext{
${ }^{4}$ CoP B also included one Area school (remote schools that cater for students from years 1-13), but only primary level teachers at this school were interviewed, and reported to be strongly engaged, in this community.

${ }^{5}$ Secondary clusters may include Area schools; remote schools that cater for years 1-13 of schooling. This was the case for COP D, which included two Area schools. Secondary school level needs strongly dominated the cluster's operations, however.
} 
A new curriculum was gazetted during the study period, with schools being given until 2010 to implement it. This undoubtedly influenced principals' attitudes towards the timeframe for change. The curriculum was strongly synergistic with the ICT PD programme's themes, placing students' needs as foremost, and positioning the fostering of key competencies at a level equal to the traditional learning areas.

\subsubsection{CoP C}

Despite the issues to do with secondary schools outlined above, data from CoP C proved useful. Like CoP B, it was in the third and final year of the ICT PD programme at the time of the interviews. It was a small cluster made up of three secondary schools situated in rural towns, around half an hour's drive from each other. I interviewed 8 participants from across the three schools. (The ninth participant was unavailable when I visited.) Analysis of interviews indicated that the new way was in the early stages of becoming embedded amongst lead teachers and principals within this community, in at least two of the three schools. However, this was at a less advanced stage than in CoP B and had reportedly impacted on smaller subsets of staff. This was evidenced by the lower extent of reported teacher buy-in, less consistency in participants' responses to interview questions, and a more diffuse sense of programme focus: Two schools had a focus on fostering student creativity through ICT and using it for selfdirected learning, but this was articulated less than clearly by some participants. The third school's focus was unclear, possibly because the lead teacher was new to the role and the principal was unavailable on the day.

Nonetheless, in terms of the progressive way in which professional knowledge was being embedded, and the different issues which had arisen at different stages, my findings about this community were broadly consistent with those for CoPs A and B. I attribute the slower knowledge embedding process in CoP $\mathrm{C}$, when compared with CoP B, to a combination of two factors. Firstly, and most significantly, CoP C was a secondary school cluster, subject to the issues outlined above. Its schools took an optin approach to ICT professional development, aiming to make initial gains amongst willing parties. The relative lack of common ground amongst its teachers meant it was impractical to focus on a specific aspect of the new way. As the facilitator explained,

The different groups are bigger and more disparate [than in primary schools]; more secular in their subject areas. To get a group of 80 teachers to read something and be interested in it is problematic. (CoP C) 
This cluster was therefore focusing on developing technological confidence and a set of implicit shared values, prior to rolling out new teaching approaches at a departmental level within schools, and across the cluster ${ }^{6}$. Secondly, CoP C had experienced recent turnover of key staff. The facilitator and one of the three lead teachers were new to their roles. The lack of continuity in these key roles had undoubtedly impacted on KT and embedding.

Despite these issues, professional change was gaining momentum in (at least) two schools in the cluster. The facilitator described the programme as instilling: (a) knowledge of technologies and how to use them, (b) knowledge about how technologies are changing society (i.e. recognising the need to change), (c) confidence knowledge ("being confident enough to have go and develop your own learning") and (d) knowledge of pedagogy - the principles and methods of (effective) teaching. This positioned the embedding of knowledge of the new way as a series of stepping-stones. At the time of my visit, those interviewed, and reportedly others within two schools, had taken several steps, moving beyond technological concerns to engage with higherlevel professional knowledge. They were also developing shared understandings about the transformative potential of this knowledge:

This year there's a definite step up with people trying out new things. I suppose it comes down to what's the nature of teaching...It's how do you think knowledge is actually transferred to kids...the process. We do try for knowledge, but it's not actually the main event any more. (Lead teacher)

This closely mirrors a comment in from a lead teacher in CoP B: "We're not trying [to instil] knowledge any more". Both statements embody a profound shift in professional values - the teacher's primary role is now seen as fostering students' skills for developing knowledge, not instilling knowledge by transmission.

Unsurprisingly, CoP B was a less vibrant online community than CoPs A and B. Lead teachers were the main participants in forums, summarising the outcomes of face-toface workshops prior to posting them online. (CoP A employed a similar approach as a prelude to broader involvement.) They also had regular videoconferences with the facilitator. The facilitator, however, engaged in considerable online communication,

\footnotetext{
${ }^{6}$ Based on interviews with facilitators, it seems that this was charactestic of the approach taken by secondary schools involved in the porgramme.
} 
being permanently connected via IM to a national group of colleagues and following the blogs of respected educators linked with the programme (members of CoP E). This informal online activity (which even occurred during the interview) contributed strongly to the embedding of knowledge in the cluster. More importantly, it contributed to my understanding of a system-level knowledge embedding mechanism that was at work within the ICT PD community. This is outlined in the macro-level embedding process outlined in Chapter 8.

\subsubsection{CoP D}

As previously noted, recruiting four suitable CoPs from the ICT PD programme for my research project proved unexpectedly difficult. Programme administrators were willing to recommend potentially suitable clusters, but lacked insider knowledge, being reliant on anecdotal reports. In hindsight I realise that assumptions about the nature of online CoPs, combined with an apparent shift towards using unofficial online communication tools (outside of the official online community space) made this task more difficult. The recruitment task was also compounded by the fact that I was seeking mature, or maturing, online communities. Perhaps there were few at this stage of development.

CoP D was recommended for inclusion based on its reputation as a mature CoP. This cluster of secondary schools ${ }^{7}$ had completed the ICT PD programme just over two years prior to the interviews, but had gained a further two years of funding for continuing its ICT-based initiatives, including professional development. The wide geographical distribution of its schools, plus reported regular inter-school videoconferencing, made it seem a likely candidate for online community activity. I interviewed nine individuals from four out of eight schools in this cluster. Two were in small settlements towns and two in provincial towns.

Unfortunately, CoP D turned out to be at an inactive stage of its lifecycle, fitting Tarmizi and de Vreede's (2005) Inactive stage and the Reflect, Self-assess and Renew stage of Snyder and Wenger's (1999) developmental model. At the time of my study, it was rolling out a business-as-usual model of technology use, with online interactions between teachers being based either around technology use or teaching management issues, such as the moderation of exam marks ${ }^{8}$. The participants I interviewed did not

\footnotetext{
${ }^{7}$ Two of the participating schools were small Area schools, catering for students across all the years of schooling (years 1-13). The cluster's focus, however, was on the needs of secondary level students.

${ }^{8}$ Teachers were rumoured to be engaging in online interactions at a pedagogical level, but none of my interviews substantiated this.
} 
substantiate external reports of higher-level professional discussions occurring online in this CoP.

Furthermore, the new way was not a shared agenda. Discussions with principals indicated that the new way of teaching was a developmental goal in two of the schools, but this was independent of the ICT PD initiative. A key participant in this CoP was a visionary who felt the CoP had lost its way. This person described the community as being in a situation of temporary stagnation, attributing this to two main factors. The first reason was the cluster's institutionalisation of a systematised, tool-centric approach to the use of ICT in teaching, leading to a loss of vision and passion:

[We hoped it would] also stimulate... some development in teacher practice....and yet....we've only scratched the surface... It's being used and it's being replicated... One of the things which makes things stick has been this process of institutionalising, but as you institutionalise, you kill the community. There's a tension there, or perhaps the passion runs out. The vision becomes harder and harder to sustain. (Visionary)

This person saw a need for a renewal of focus, combined with a shift to higher order professional knowledge, in order to sustain the CoP:

We're almost at a stage where if [this cluster] is going to go anywhere, there almost has to be this quite drastic shift of focus onto teaching.... We're starting to try to put together ideas we can use, which are going to be more focussed on the teacher, and pedagogy. (Visionary)

The second factor impacting on stagnation was the loss of the facilitator whose role it had been to connect people and ideas. The nature of this gap became clear when I interviewed the cluster's technical leader, who was now at the hub of the community. This person's technical focus, combined with a heavy workload and personal preferences, was leading them to actively avoid professional dialogue:

I really restrict my own inputs. If I have too many, life gets silly. So I refuse to be part of too many on-line e-mail groups. I don't want to have blogs and wikis and those sorts of things running... if people want to contact me, I've got e-mail, I've got my phone, and those are enough inputs. People often say, "Can I have your Skype address?" and I say, no, you can't. That's my personal life...

Interviewer (IV): So your role in making those communities work is to exclude the distracting other things?

Yes. I have to. If I didn't, I wouldn't get this job done...." (Technical leader) 
The fact that the technical leader had to work so hard to avoiding becoming a de facto facilitator is indicative of a demand for a connector in this community. The absence of such a person appeared to be a profound inhibitor of KT, with knowledge becoming trapped in a bottleneck:

There's probably not much [knowledge] flowing into me. It's probably a one-way flow out of me into the other people. (Technical lead)

Unfortunately, owing to the inactivity of this online CoP and its lack of focus on the new way, much of the data from CoP D lacked usefulness. However, the visionary's opinions about the need for CoP renewal following institutionalisation (the embedding of knowledge in a rigid way; seen as a barrier to further CoP-based developments) were highly useful. The data contributed to my development of a cyclic, rather than linear, model for how knowledge embedding can be facilitated by online CoPs at the organisational level. In addition, the situation outlined by the technical leader strongly supported my findings from other communities about the macro, or system, level knowledge embedding process; in particular, the vital role played by connector-leaders.

In retrospect, I recognised a methodological problem in having included CoP D. This cluster had been in an earlier cohort of ICT PD clusters than the others in this study. Owing to its having been funded for a further two years of ICT professional development, it appeared to be on a par with CoPs A, B and C. However, there had been no formal link with the ICT PD programme for two years. In the meantime, the ICT PD programme had developed a synergy with the objectives of the emerging curriculum, resulting in an emphasis on transforming teaching in line with the new way. There may have been a different outcome for CoP D had it embarked on the programme two years later.

\subsubsection{CoPE}

My discovery of CoP E more than compensated for this above problem. I had not included this CoP in my original research design and was unaware of its existence until after entering the field. Even once I encountered it, two assumptions got in the way of my recognising it as an online community: Firstly, I assumed that any CoPs relevant to this study would be formally linked to the ICT PD programme, and secondly, I assumed that online CoP were facilitated, with a centralised record of discussions. I had to revise both assumptions. 
The first sign of CoP E was my discovery that key individuals in these CoPs relied on blogs for keeping their professional knowledge current. One member of CoP A, two of CoP B, and one of CoP $\mathrm{C}$ authored their own professional education blog (edublog) and commented on the blogs of others. One further member of CoP A, six of CoP B, and one of CoP C were regular followers of one of more edublogs. The blogs these people followed belonged to respected educators involved in the ICT PD programme typically, but not always, cluster facilitators or lead teachers.

Upon consideration, I realised that this blog-based activity was a sign of dual community membership, or inter-community boundary spanning, on the part of the individuals concerned. I revisited the literature and found an emerging set of research that extends the traditional definition of online communities to include communities of reciprocally linked individuals within high-density areas of blogging networks (Efimova \& Hendrik, 2005; Hodkinson, 2006; Wei, 2004). CoP E was such a community. My analysis of blog and Twitter linkages (see Appendix F) showed strong reciprocity amongst those who had a visible online presence. (CoP E was the NZ-based subcommunity of a community of strongly inter-connected bloggers that sat within the edublogging network. Owing to the case boundaries, I have simplified the way I present this layered external structure, in most cases simply referring to it as a network.) I was fortunate in that teachers' restricted view of what constituted an online $\mathrm{CoP}$ had led me to revise my initial questioning approach, asking about their use of diverse online communication tools. This helped me to uncover the community.

CoP E was a non-official, highly active Web 2.0 based online CoP, located within an international network of edubloggers - teachers who interact via blogs (and other online tools). It could also be termed a Community of Passion: Its core members were converts to the new way, believing in the transformative power of ICT when used appropriately to support student-centred learning. They made intensive use of a wide range of online communication technologies to sustain their beliefs, enrich their understandings, and promote and broker knowledge. In Chapter 8, I describe their role as connector-leaders.

Those people who followed, but never (or rarely) authored or commented on, the blogs were peripheral members of CoP E. In Chapter 8, I describe their role as followerfeeders. These people were reluctant to leave a visible online record, typically seeing themselves as being at a lower level than the core members ("I'm at the bottom"; "They're way up there"). They therefore corresponded with those whom they followed 
via invisible, or behind-the-scenes means, via IM and e-mail. While connector-leaders kept in touch with each other via tagging, alerts, RSS feeds, and Twitter, they were aware of their followers' communication preferences, proactively feeding them relevant information (with links to URLs) via IM or e-mail.

My decision to amend my research design to include CoP E was more significant than I initially realised. As I interviewed more of its members ${ }^{9}$, I saw that this community operated at a different level to the others. Through its overlapping membership with cluster CoPs, it facilitated KT in clusters, while indirectly connecting clusters across the programme. It also acted as a middle layer community of knowledge brokers; forming as a bridge between the cluster communities and an international network of experts and practitioners whose ideas its members followed. Rather than viewing the case as a set of parallel subunits, I came to develop a more complex, system-based perspective of how online CoPs were facilitating the embedding of knowledge. I realised that I could better address the research question if I moved beyond considering only the meso-level perspective that was most obvious in interviews - a view of how embedding was occurring within schools and cluster - to also consider the macro-level perspective - how embedding was influenced by interactions between the different communities and levels of communities. This emerging system-based perspective was strengthened by my recognition, during the later stages of data analysis, of an additional micro-level perspective - the way in which online CoPs facilitated knowledge embedding at a personal, individual level. My system-based perspective on the case led me to develop a multi-level theory.

Figure 15 shows the structural relationships between the five online CoPs I studied, showing how CoP E (centre) acted as a connecting community. Three of the four official, cluster-based ICT PD communities (CoPs A, B and C) had overlapping membership with CoP E, a non-official, blog-centric community of connector-leaders (shown as the central circle). The overlapping membership occurred in two zones: (a) a central visible zone (the central shared area) within which the core members, connector-leaders, interacted with a publicly visible online presence and (b) a peripheral invisible zone (the lighter area around the core) within which followersfeeders interacted invisibly, via IM, email and phone; and face-to-face, with the

\footnotetext{
${ }^{9}$ Once I had identified COP E, I interviewed three further members who were in clusters not already in the sample and gathered data from the community's publicly available online records.
} 
connector-leaders. My information about follower-feeders' activity in CoP E came from my interviews with them and with the connector-leaders they followed. Although invisible in CoP E, follower-feeders had a visible role in their respective closed, clusterbased online communities. They fed the knowledge they gained through their participation in CoP E to their cluster-based peers using both online and offline means.

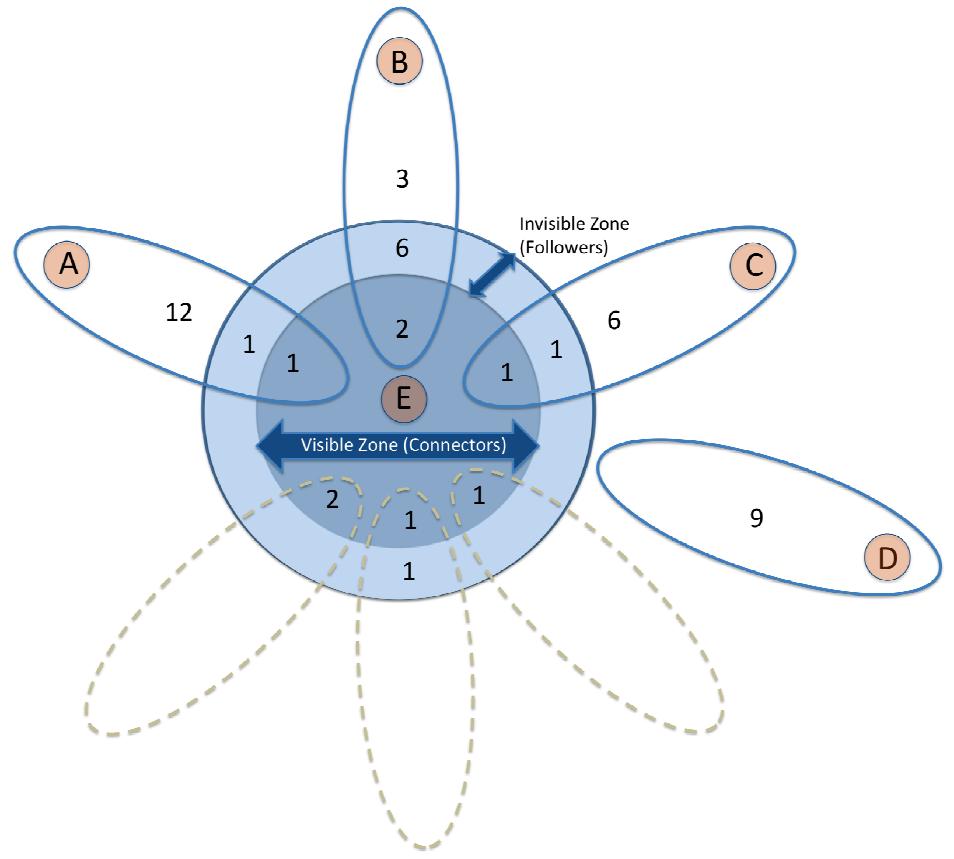

Figure 15: Participating CoPs, showing overlapping membership with CoP E

One member of CoP A, two of CoP B and one of CoP C were connector-leaders in CoP E. I gained access to considerable valuable online data from these members, including blog posts, lists of other bloggers whom they followed, Twitter records, Del.icio.us records (social bookmarking of content), Teacher Tube videos, podcasts, and material from other contexts, such as Second Life and Linked In, that had been cross-posted onto blogs. I gained further data from the public online records of selected other members of CoP whom these people interacted with at a national and international level.

One member of CoP A, six of CoP B and one of CoP C were invisible follower-feeders in CoP E. The programme leader and a national facilitator, who I had interviewed earlier, were also members of CoP E (as a follower and a connector respectively). The dotted lines in figure 15 indicate communities outside the scope of this study, to which the additional members of CoP E whom I interviewed belonged. 


\subsubsection{Overview of theoretical findings}

The key outcome of this research project is a three-level theory to explain how online CoPs facilitated the embedding of transformative professional knowledge in the research setting. This theory comprises three complementary embedding-facilitating mechanisms that operated simultaneously at the micro, meso and macro levels (see figure 16). In addition, I identified five fundamental knowledge embedding processes that promoted embedding and were facilitated by the mechanisms. I firstly introduce my findings about the five fundamental processes, then summarise the three-level theory.

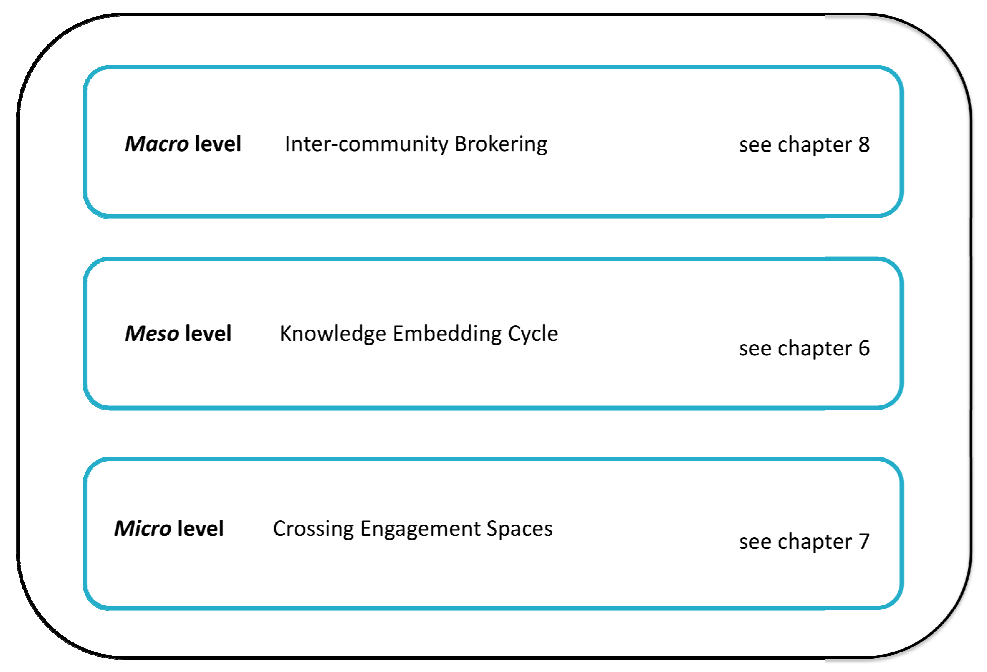

Figure 16: Three-level theory: micro-, macro- and meso-level facilitating mechanisms

\subsubsection{Five fundamental knowledge embedding processes}

Five fundamental knowledge embedding processes facilitated the embedding of the new way within the case: focusing, persuading, aligning, adapting, and owning (developing ownership). These processes were not exclusive to the online realm, but were driven, or catalysed, by the facilitating online CoP mechanisms at micro, meso and macro levels. The five processes are a unifying, recurring theme in Chapters 6-8. I derived them from the iterative aggregation of many categories of embedding-related codes.

\subsubsection{Focusing}

Focusing involved having a focus on (a) specific approach (e.g., a learning model, teaching method, or use of technology) that was intended to engender understanding of the new way; and/or (b) a specific issue related to implementing the new way. 
A school-wide focus, such as inquiry learning, thinking skills, student blogging, and IMbased student-to-student mentoring, provided a shared container or boundary object (Carlile \& Rebentisch, 2003; Pawlowski \& Robey, 2004) for negotiating meaning. It increased common ground, fostered collegiality, made change more manageable, and promoted the development of mutual understanding. CoPs $\mathrm{A}$ and $\mathrm{B}$ had focused in a sustained way on developing inquiry learning models, resulting in successful embedding. The more diffuse focus in CoP C seemed to be associated with a slower embedding process and more uneven results.

In order to successfully embed the new way, teachers also needed to focus on identifying and resolving specific issues relating to practice or theory. Having a sense of focus helped them stay on track, avoid being captured by 'the lure of technology' per se, and screen out non-relevant online content. The ways in which online CoPs facilitated focusing are outlined in Chapters 6-8, in relationship to each embedding mechanism.

\subsubsection{Persuading}

Persuading involved being persuaded, persuading oneself and persuading others of the rightness of the new paradigm and its superiority to the professional status quo.

Embedding of the new way required substantial change on the part of many teachers and schools. Persuading oneself and others of the need to change, and of the relevance and veracity of the values and beliefs associated with the new way was therefore an important aspect of embedding process. Persuading facilitated the reframing of mental models, engendering belief in the new paradigm. It inspired motivation to change, justifying the effort required to embark on change and engage with new knowledge. It also fostered the resilience needed to continue when things became difficult.

The cultures of use associated with forums and blogs encouraged teachers to use persuasion in their reflections and arguments. Individuals also persuaded one another via IM and Twitter. The diverse ways in which online CoPs facilitated persuading are outlined in Chapters 6-8, in relationship to each embedding mechanism. 


\subsubsection{Aligning}

Aligning involved (a) aligning emerging practices, experiences, interpretive frameworks, values and beliefs with those of peers and the community, and (b) aligning practice with theory that supported the new paradigm. This included reinterpreting existing practice in light of new theory, adjusting practice to better align with theory, and adjusting personal theory to align with emerging practice.

Collegial alignment of emerging understandings and practices helped build shared cognitive bases, increasing absorptive capacity; the ability to absorb new knowledge (Rolland et al., 2003, Szulanski, 2000). Alignment also provided reassurance about being on the right track, and helped legitimise and stabilise new knowledge.

The desire to align fostered relationships of mutual support: Teachers were comforted by the fact that they were caught up a common change process ("We're all in the same boat'). They were reassured by their recognition that they shared interests and problems with others, and relished the opportunity to tackle these in partnership. By discussing common themes, CoP members developed an aligned language and aligned ideas about what mattered. Teachers regularly tested out the fit of their emerging ideas with colleagues, keeping each other on track. They benchmarked their own progress against that of colleagues and emerging community norms. In instances where individuals challenged the emerging paradigm, facilitators intervened to bringing the resistant individual into alignment.

As the new way was embedded, the alignment of theory with practice and of practice with theory became an ongoing challenge. This iterative process gradually reduced dissonance and increased acceptance. The ways in which online CoPs facilitated aligning are outlined in Chapters 6-8, in relationship to each embedding mechanism.

\subsubsection{Adapting}

Adapting involved (a) adapting and modifying established practice, ways of thinking (interpretive frameworks) and values to accommodate emerging beliefs and norms, (b) adapting new approaches (models and methods) to better suit the organisational and individual context, and (c) adapting communication norms to a community-based approach.

New models and methods needed to be adapted so they better fitted participants' contexts, whether a particular age group of students, a department or syndicate, or a 
school. Adapting improved the fit of models and by customising them to local needs schools increased their stickiness (Szulanski, 2000).

The process of adapting was gradual, beginning with reframing of the status quo and committing to change. It involved piloting a new method then gaining fluency, modifying the method so that it was more practical. Once individuals gained fluency, they began to internalise their knowledge but continued adapting by improvising and making gradual refinements. Online CoPs facilitated adapting by providing new contexts for reflection, problem solving and mutual support. In CoP A, Prensky's (2005-6) metaphor of the teacher as digital immigrant was taken from online forums into workplace discussions and jokes, normalising the difficulties of adapting. The ways in which online CoPs facilitated adapting are outlined in Chapters 6-8, in relationship to each embedding mechanism.

\subsubsection{Owning}

Owning involved developing ownership of new knowledge and associated interpretive frameworks, practices, beliefs and values at the individual, school and/or community level.

Owning knowledge was necessary in order for it to 'stick' in schools and to become part of an individuals' own repertoires of practice. Knowledge that was strongly owned is knowledge that has been invested in. The new way - encapsulated in various teaching approaches and methods - was embedded in, and valued by, those with a sense of ownership, whether at the level of the individual, organisation or community.

The process of owning began with committing to change (Coming on board) and buying into a new vision. It continued with problematising - the articulating and owning of specific problems relating to change - and the borrowing and trialling of new models and methods. Contextualising new knowledge by customising it (at the organisational level) and personalising it (at the level of the individual) strongly facilitated owning. In schools, advanced ownership was associated with institutionalising and guarding of their customised models. For individuals, owning also involved believing in the new way. Owning was strongly facilitated by the micro-level mechanism of crossings. The ways in which online CoPs facilitated owning are outlined in Chapters 6-8, in relationship to each embedding mechanism. 
I now provide an overview of the meso-, micro- and macro-level embedding-facilitating mechanisms that make up the three-level theory.

\subsubsection{Meso-level facilitating mechanism: Knowledge embedding cycle}

At the meso, or organisational level, the embedding of transformative professional knowledge was driven by a six-stage knowledge embedding cycle. This cycle comprises six stages of an organisational change journey that occurred as the new way of teaching became progressively embedded (see figure 17). Each stage was characterised by a distinct set of activities and issues, with online CoPs playing an important role in addressing issues and facilitating progression through the cycle. The six stages of the knowledge embedding cycle are: Plotting the Course, Coming on Board, Setting Out, Staying on Course, Anchoring and Settling. The names of the stages reference the metaphor of a professional change journey that had strong currency amongst participants.

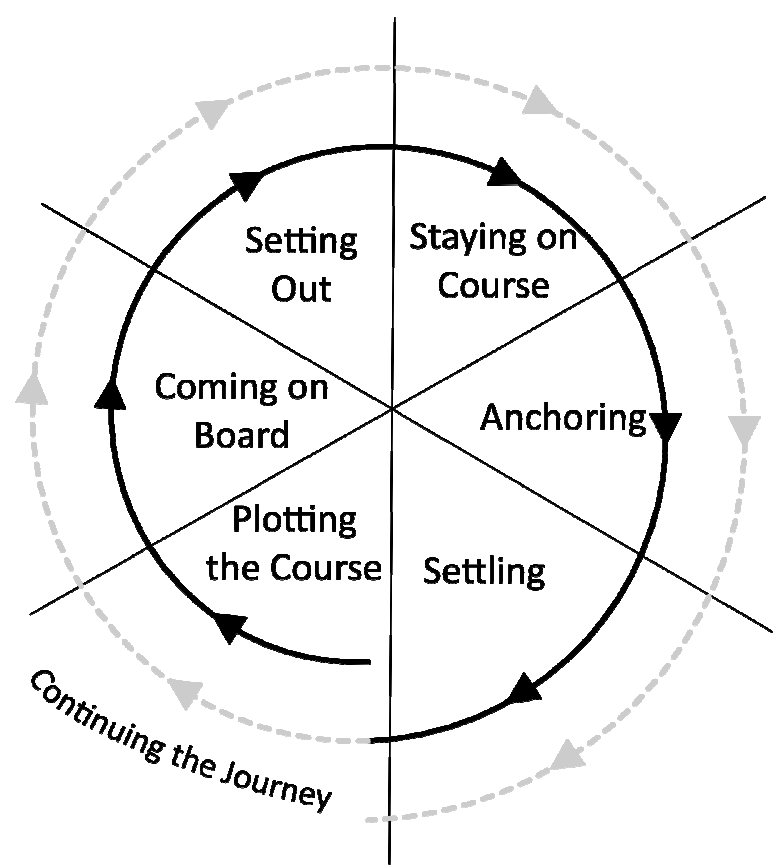

Figure 17: The six-stage knowledge embedding cycle

Completion of all six stages constituted the end of an embedding cycle, resulting in the routine use of a new model or method that encapsulated the new approach, bound up with a complementary new framework of thinking.

Unlike many models of organisational change, this model is not linear, but cyclic. This arises from the context for this study, which was one of transformative change. 
Embedding of a new paradigm is known to be contingent on a change in people's belief structures and interpretive frameworks (Handal, 2004; Keys, 2006; Richardson \& Placier, 2001). At participant check workshops, several individuals emphasised the view that that once an embedding cycle was completed, additional embedding cycles were needed. While a method encapsulating the new way could become embedded in the course of a single cycle, the new way cannot would not itself become fully embedded until several cycles, with new, complementary foci, had occurred. The meta-model for the embedding of transformative professional knowledge at the meso level is therefore a spiral in which each successive cycle wraps around and reinforces the previous cycle. The embedding of knowledge is a process that is never entirely complete, but is characterised by the ongoing deepening and enrichment of understanding and increasing personalisation of practice. The knowledge embedding cycle is outlined in depth in Chapter 6.

\subsubsection{Micro-level facilitating mechanism: Crossings}

At the micro, or individual, level the embedding of professional knowledge was driven by individuals' crossings between diverse online and offline engagement spaces in a complex, polycontextual system. CoP members interacted within a complex sociotechnological system made up of multiple, diverse engagement spaces, or communication contexts. Online forums, IM, blogs and Twitter supplemented the traditional offline engagement spaces of the classroom and staffroom, creating a new, multi-faceted arena for professional reflection and interaction. This was a situation of (hyper) polycontextuality (Engeström, et al., 1995; Goodwin, 1990; Reder, 1993); multiple micro-worlds or participation frameworks, within and across which individuals operated.

Different engagement spaces provided different benefits and had different cultures of use that governed the agenda, language, tone, style and register of communications. For example, forum culture promoted self-analysis and the making of resolutions, while IM culture valued more honest expression of concerns, seeking of clarification, critiquing of events, testing out of ideas, and prodding of peers. The embedding of knowledge was facilitated by individuals' ongoing crossings between these diverse engagement spaces, with the multiplicity of engagement spaces acting as a catalyst to the consolidating of ideas: 
I spend a lot of time crossing from environment to environment. Professionally these crossings help to consolidate my understandings and...give me that little bit more understanding I need to be able to take the ideas into my classroom. (Teacher, CoP E, wiki)

As teachers undertook crossings, driven by various needs, they crossed between different communities, roles, literary genres and social conventions; between synchronous and asynchronous modes of communication, online and offline contexts, and work and home. In doing so, they needed to constantly translate, interpret, reinterpret, adapt, combine, argue, revisit, analyse, justify and re-articulate the ideas they engaged with, while differentiating their contributions. (For example, a blog posting might draw on an idea stated in an online forum, but it needed to be more expansive, argumentative in structure and authoritative in the new context. Supporting examples could be gathered from colleagues via IM or Twitter.) The significant effort involved in this continual re-contextualisation led to strong personalisation (Nonaka \& Takaeuchi, 1995) of the ideas, theories, methods, values and beliefs with which individuals engaged, and a high level of ownership of this knowledge.

Embedding of the new way was also facilitated by theory-practice crossings; i.e., crossing between reflective engagement spaces (e.g., forums, blogs) and active ones (notably the classroom). This movement fostered the iterative alignment of practice with personal beliefs:

It sort of firms up what you believe, and then you look at what you're doing, and so you change that.... (Lead Teacher, CoP A)

Efforts to reconcile the theoretical and practical dimensions of knowledge are known to play a key role in the development of professional understanding in teachers (Bromme \& Tillema, 1995; Leinhardt, et al., 1995).

While individuals undertook different trajectories of crossings, they did this within an environment in which there was a broad convergence of knowledge. Polycontextuality provided a setting in which influential themes were readily distributed and magnified. Memorable themes, metaphors and "threads of ideas" were recycled and varied until they permeated many different engagement spaces. This, combined with social norms of alignment, promoted the convergence of beliefs and interpretive frameworks at community level. It also ensured that the knowledge being embedded through personalised crossings was in keeping with the new way. As one participant said, 
My learning is a dialogue. I read, comment, cross-comment, post, hyperlink and think and link. The views of others influence my thinking Through this dialogue I have to re-define my thinking. By justifying why I think the way I do I clarify in my own head what it is that I do actually believe. (Teacher, CoP E)

$\mathrm{KM}$ research has highlighted the importance of boundary crossing in KT (Ancona \& Caldwell, 1992; Harada, 2003; Pawlowski \& Robey, 2004). The boundary spanner is seen as facilitating the adaptation and translation of knowledge into new contexts. The micro-level facilitating mechanism extends this theme, viewing individuals' ongoing crossings between engagement spaces as a strong facilitator of personal knowledge embedding. The micro-level mechanism is the subject of Chapter 7.

\subsubsection{Macro-level facilitating mechanism: Inter-community brokering}

At the macro, or system of communities, level, embedding of the new way was driven and sustained by inter-community knowledge brokering. CoP E played a key role in a knowledge value chain. It formed a hub to CoPs A, B and C, indirectly connecting them, while acting as a middle layer community between them and a global network of edubloggers, to which its core members belonged (see figure 18). This structure exemplified Castro's CoP ecosystem of overlapping online conversation spaces (2004, 2006), but was more complex in that it was not only overlapping, but also tiered, like a natural ecosystem.

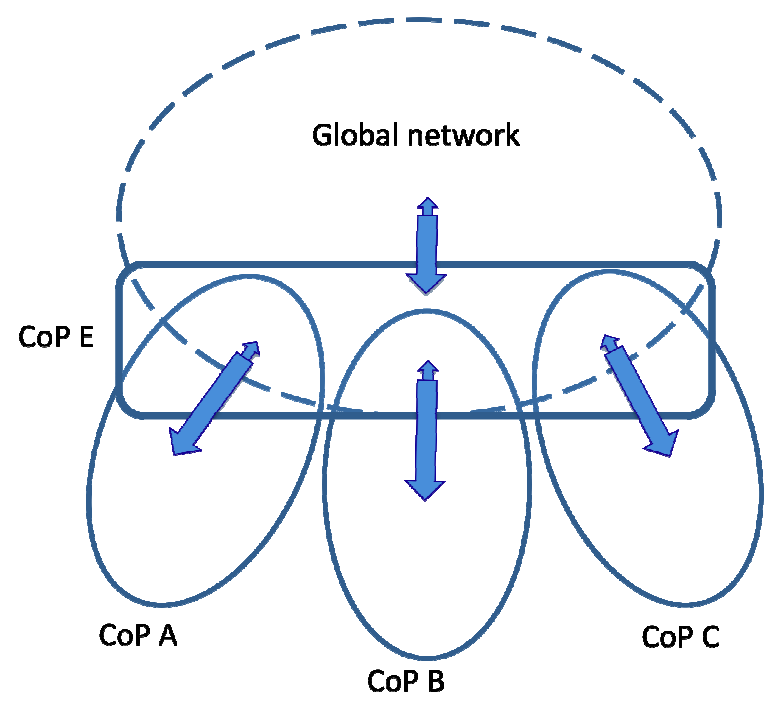

Figure 18: The macro level perspective - CoP E as a hub and middle layer community

CoP E was a community of passion whose members strongly supported the new paradigm. It brokered knowledge both 'downstream' and 'upstream', but knowledge 
flows were the strongest in the downstream direction (i.e., from CoP E to CoPs A B and C) owing to the high level of online activity of its core members and their role as connectors, their status as leaders, and their commitment to a cause: Those whom I interviewed could be seen as missionaries for the new way.

Brokering was performed by members of CoP E who played two non-official knowledge broker roles: visible connector-leaders and invisible follower-feeders. Connectorleaders were respected, well-connected educators who shared a belief in the potential of ICT for enhancing learning if used in a student-centric way. In CoP E they had a high level of visibility, authoring blogs and commenting on the blogs of others. (They also interacted less visibly using IM, Twitter and e-mail.) They identified strongly with this CoP:

(When I had a problem) my blogging community was the one that made me feel better. It wasn't my colleagues in the classroom next door, because they couldn't offer me any advice... There isn't anyone who's as passionate about the technology in the classroom as I am." (Connectorleader)

In their cluster CoPs, connector-leaders played formal or informal leadership roles, selecting discussion themes, facilitating forums, promoting methods, offering support and/or running workshops. Their connections in the global network provided access to a wide range of knowledge, and they followed and communicated with some of its members. Connector-leaders fostered the embedding of knowledge in ICT PD clusters through their connections with follower-feeders - peripheral members of CoP E who were core members in their cluster CoPs (CoPs A, B and C). Follower-feeders followed one or several connector-leaders' blogs and communicated with them invisibly via IM.

Connector-leaders had a strong persuasive influence. They filtered incoming knowledge from the global network, seeking out themes that supported the new way and were relevant to clusters' needs. They varied these themes in their blogs, personalising and localising content in a way that increased relevance to their followers. They also fed this content to their followers via IM and email. By echoing, varying and remixing a set of recurring themes they reinforced and amplified them, promoting alignment of thinking at system-level. In addition they provided a matchmaking service, linking followers to others with specialist knowledge. This was enabled by a culture of mutual $24 \mathrm{X} 7$ support. Connector-leaders also moved knowledge "upstream" to the global network, for example, featuring examples of novel student-centred classroom practice in their blogs. This enhanced their street-cred in 
the blogging network, building readership and raising the quantity and quality of engagement with their blogs, which in turn built value for their followers.

Follower-feeders were also important knowledge brokers, despite their invisibility in CoP E. Within their schools they were "leading lights". They participated in online forums and IM discussions, but also spent considerable time engaging with their own followers - regular teachers - in offline contexts. They extended the reach of connector-leaders and amplified their influence by seeking and receiving their advice, adapting their ideas, and feeding them on to regular teachers. This 'downstream' flow of knowledge was like a food chain:

I take up her ideas, and I'm not confident enough to give things back. But I am passing it on to people below me. There are probably people feeding off me who will never go on-line, so I have to go out seeking more to give to them (Follower-feeder).

Follower-feeders brokered the transfer of knowledge across the online-offline boundary of their cluster CoPs. Regular teachers spent little time online, so follower-feeders filtered content from the connector-leaders they followed with their needs in mind:

I check things out prior to telling staff. I guess I make decisions about what will work, and what not to tell them, also. (Follower-feeder)

Two ecological themes arise from the macro-level perspective: The first is a knowledge food chain (or value chain) in which knowledge was passed on, reconstituted and reused by different levels of consumers. The second ecological theme is that of symbiosis. Connector-leaders and follower-feeders performed symbiotic, mutually dependent roles: Follower-feeders relied on connector-leaders as purveyors of quality, for keeping their ideas current and relevant, for solving problems, and for connecting them to others. Connector-leaders gained status and recognition from this relationship, as well as access to practical knowledge that could enhance their credibility in the network. Figure 19 shows the interaction of Connector-leaders, follower-feeders and regular teachers and the different boundary zones across which knowledge brokering took place. I explain the macro-level mechanism in depth, with examples, in Chapter 8. 


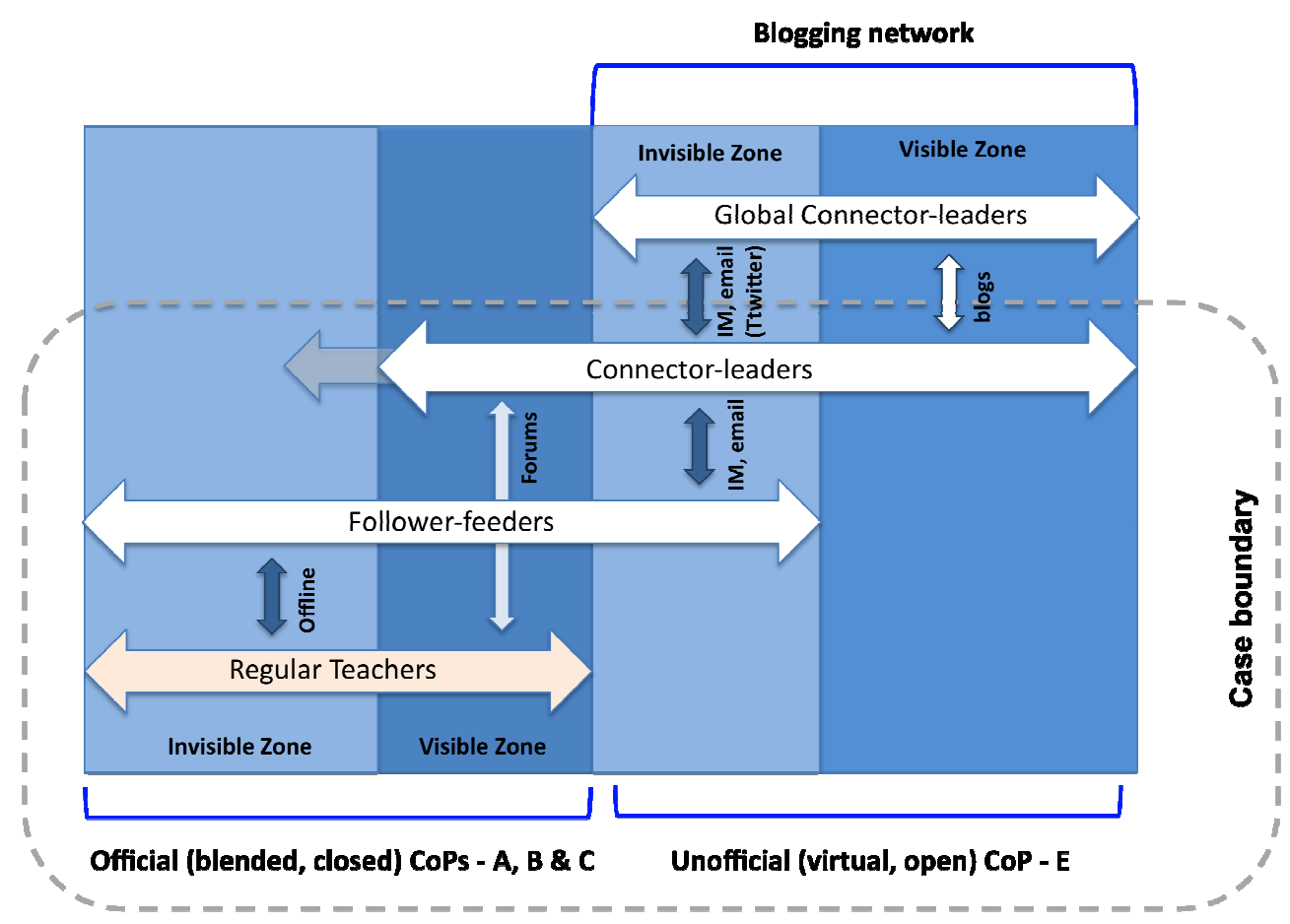

Figure 19: Interaction of connector-leaders, follower-feeders and regular teachers showing boundaries

\subsection{Chapter summary}

This chapter has provided an overview of my research results - five fundamental knowledge embedding processes and a multi-level theory comprising three complementary embedding-facilitating mechanisms that operate at the micro, meso and macro levels. In the next three chapters I discuss the three facilitating mechanisms in depth, with supporting evidence.

Chapter 6 provides an explanation of the meso, or organisational, level embedding mechanism, the knowledge embedding cycle. I deal with this mechanism first because it was the first explanation that arose from my analysis of the data. (The cycle has six stages, so Chapter 6 is also the longest chapter.)

The micro- and macro-level explanations for how online CoPs facilitated KT and embedding were less immediately visible, requiring more analytical and interpretive effort on my part, but they were equally important to the embedding process. Chapter 7 outlines the embedding-facilitating mechanism of crossings operating at the micro, or individual level. Chapter 8 outlines my explanation for the macro, or system level knowledge embedding mechanism. In the final chapter I summarise the limitations and implications of this study. 


\section{The meso-level knowledge embedding cycle}

\subsection{Introduction}

In this chapter I outline my meso-level explanation for how online CoPs facilitated the embedding of knowledge. The meso-level perspective is concerned with explaining the process of change within schools, taking into account the experiences of individuals and their interactions with others in the CoP. At this level, the transfer and embedding of knowledge followed a series of distinct stages, which I have represented in the knowledge embedding cycle (KEC) - figure 20. This model provides a useful framework for interrogating the role played by online CoPs in embedding the new way in participating schools. Its intent is to identify essential patterns and experiences related to the knowledge embedding, rather than to reflect the path taken by a particular school or group of schools.

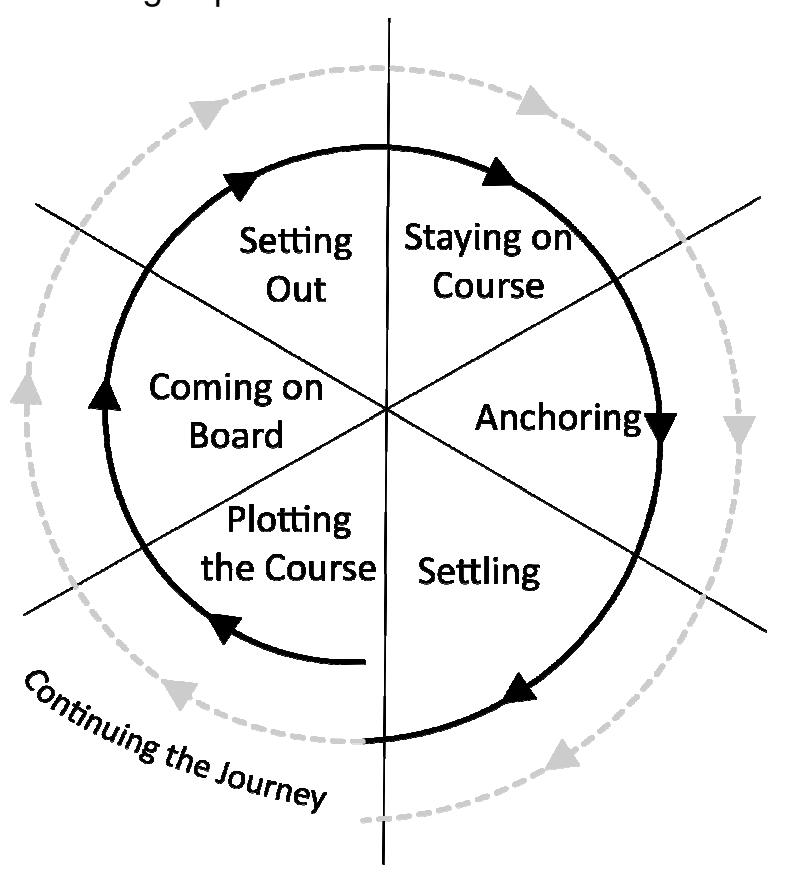

Figure 20: The knowledge embedding cycle (KEC)

The KEC portrays the embedding process as a transformative change journey that occurred in stages, based around school-specific foci, over a three-year period. Six stages of change occurred as the new way of teaching became progressively embedded in schools: (1) Plotting the Course, (2) Coming on Board, (3) Setting Out, (4) Staying on Course, (5) Anchoring and (6) Settling. The names of the stages reference a journeying metaphor that was in common use amongst participants and which fostered their acceptance of change. Each stage was characterised by a distinct 
set of issues and activities (or embedding sub-processes), as knowledge of the new way became progressively embedded. These activities contributed to the five fundamental embedding processes - persuading, aligning, adapting, focusing and contextualising.

Although the stages appear in the model as cleanly delineated, in reality there was some fuzziness between the end of one stage and the start of the next. For example, Coming on Board involved building commitment to change. It overlapped with the next stage, Setting Out, as teachers came on board at different rates. In addition, although the cycle has six equal segments this does not mean that equal amounts of time were spent in each stage.

Completion of a KEC resulted in a school's (and its teachers') routine use of a model or teaching approach that had come to embody that school's perspective on the new way. The school's use of this model or approach was bound up with a set of new norms and understandings - a blend of theories, values and beliefs and interpretive frameworks about the teacher's role and the nature of the teaching and learning process. Once embedded, this amalgam of knowledge of the new way was valued, believed in, understood and owned by schools and their staff members. It was used with growing fluency, resulting in satisfaction, pride, improved professional understanding, and a sense of belonging to a new professional order.

However, the new paradigm was not itself fully embedded in schools at the end of one KEC. The final stage, Settling, when a school's chosen approach was being institutionalised, was seen by participants as a time of risk: Over time, it might result in stagnation. Individuals at the participant check workshops emphasised a need for schools to undertake further KECs, with new foci that were compatible with the new paradigm. Successive cycles were also needed to bring latecomers on board. The meta-model for sustained embedding is therefore a spiral in which each successive cycle enfolds the previous one, building on and re-embedding its essence.

In this chapter, I firstly discuss why the meso-level perspective was the first one to emerge from my research project. I then outline a key contextual factor impacting on knowledge embedding at meso level: workplace leadership. Following this, I recount the evolution of the KEC. I then outline each stage in depth, discussing the issues associated with each, describing the activities involved, and considering how (and whether) online CoPs contributed to them. 


\subsection{Contextual issues}

Two contextual issues stand out in relationship to the meso level of analysis. These are (a) the perspectives and roles of the research participants and (b) the importance of strong leadership in the workplace.

\subsubsection{Participants' perspectives: facilitating a meso-level view}

In the interviews, participants placed a strong emphasis on describing change that had occurred at the level of the school. I saw this as arising from a combination of factors: Around two-thirds of the study participants held roles that involved some responsibility for school-level change. Others were enthusiasts for the new way, acting as unofficial change agents. This could be interpreted as a meso-level bias in the participant group, arising from the fact that schools had brokered the selection of participants for the study. Because change agents had played the most active roles in the online community, they were a natural choice for this study, which focused on the how of online community workings. In addition, the ICT PD programme itself had a meso-level focus: its key outcome was change at school level, with reporting undertaken on this basis. Given these factors, it is unsurprising that the meso-level perspective was the first to emerge from my analysis of the data. It was easier to identify and it required less analytical and interpretative input than did the micro- and macro-level views.

\subsubsection{Organisational leadership}

The second contextual issue I observed was that successful knowledge embedding at the school level was associated with strong and persistent organisational leadership. New knowledge had been most broadly embedded in schools where principals had treated the ICT PD programme as a change exercise, prioritised it, made participation (including online participation) mandatory, formed change-based allegiances, contributed to professional dialogue and actively countered resistance ${ }^{10}$. Doing all of these things required a major investment of leadership energy:

Once we got into it we realised that it was a bit bigger monster than we'd realised. It needed a lot of leadership to keep things going along (Principal, CoP A)

Strong leadership was undoubtedly a critical enabler of knowledge embedding at the organisational level. However, most principals had a low level of online community

\footnotetext{
${ }^{10}$ In one small school, close to fifty percent of staff; those most resistant to change, had resigned during the programme, underlining the fact that it was not a flavour-of-the-month exercise. The principal had leveraged this opportunity, recruiting staff with the skills to consolidate the gains made.
} 
engagement. Because the focus of my research is on online CoPs, I do not explore the role of the principal in depth.

\subsection{Developing the knowledge embedding cycle}

I developed the model of the knowledge embedding cycle from the iterative analysis and synthesis of data from individuals in CoPs $A, B, C$ and, to a lesser extent, those in CoPs D and E. In the schools in CoPs A, B and C, it was clear that a professional change journey had occurred, and/or was still occurring, Participants described the embedding of knowledge as having taken place gradually, over the last 2-3 years. Their accounts and the online records indicated that schools had faced different challenges over this time. I found there were commonalities in the kinds of issues they had faced at different stages. I grouped the reported issues and activities into stages, hoping that understanding their sequential structure would aid my investigation of how online CoPs had facilitated (or had not facilitated) the knowledge embedding process over time.

Despite the commonalities in the reported sequence of events, teachers at a given school did not necessarily see themselves as being at the same point of the change journey. In schools in CoP A, which had completed the programme, some teachers spoke of being in a final settling or catch-up period, but others spoke of having moved on, using their new knowledge as a kind of launch-pad for exploring a new method that supported the new way. I saw this as an indicator that the embedding process was not linear, but cyclic. I developed three iterations of a model that built on this concept of a cyclic process.

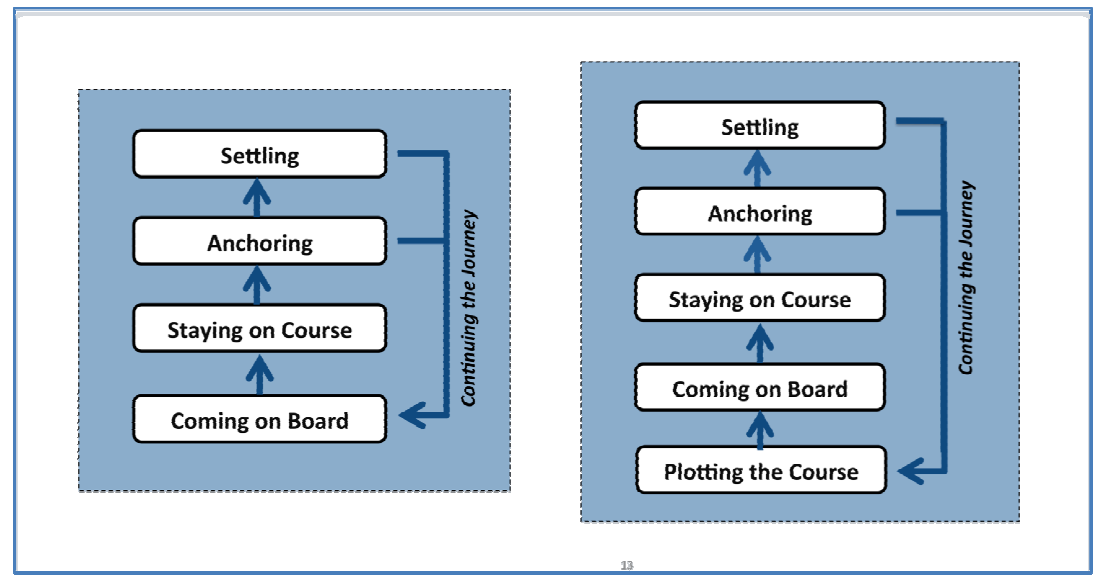

Figure 21: Knowledge Embedding Process - Iterations 1 and 2 
The first two iterations are shown in figure 21. I initially grouped the activities into four stages then later added the Plotting the Course stage to acknowledge the influence of planning for change. Both iterations had an optional loop back to the start prior to the stage when institutionalisation (Settling) occurred. This allowed for some teachers to move on before others. Selected members of all five CoPs attended participant check workshops and contributed feedback on the second version via an anonymous wiki. Based on their feedback, I developed the third and final version (figure 22).

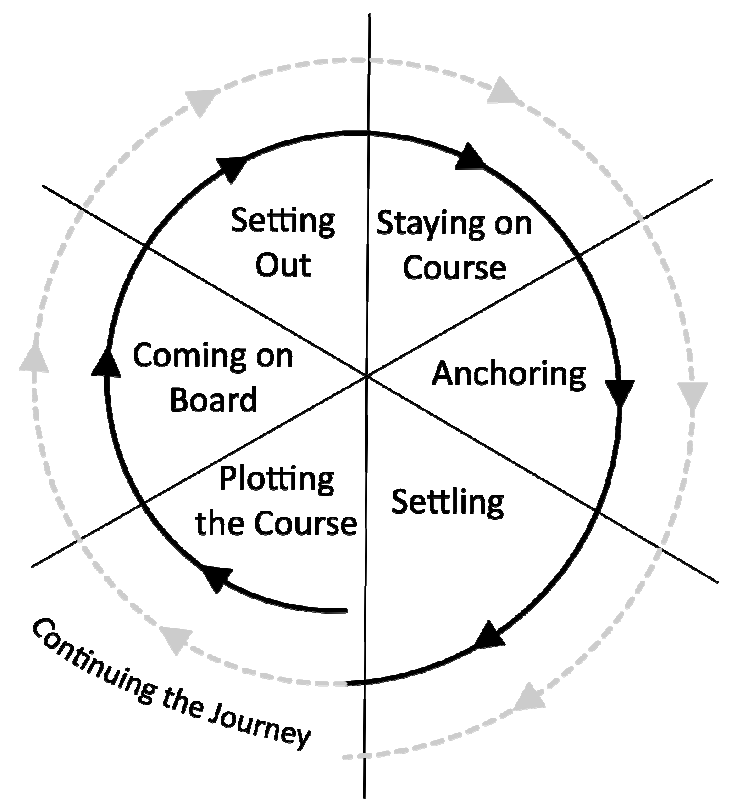

Figure 22: Knowledge embedding cycle: final spiral-based model

This version uses a spiral to portray the cyclic nature of the process, showing successive cycles enfolding one another. (The duration of my study did not allow me to see the completion of more than one KEC, but new cycles had begun in some schools.) This version also has a new stage called Setting Out. I added this to differentiate the issues encountered in the early stages of change from the multitude of issues occurring later, during Staying on Course. I also removed the optional shortcut from Anchoring to the start in this version. Instead, Settling is seen as flexible in duration, allowing for change agents to move ahead of others and initiate new cycles.

\subsection{The knowledge embedding cycle}

I now outline the six stages of the KEC. I discuss the issues faced and activities undertaken at each stage and consider the role played by online CoPs in facilitating the embedding of knowledge. For each stage, I provide a table summarising the activities involved and indicating how (and whether) online CoPs facilitated them. A summary of 
all the activities, and their relationship to the five fundamental knowledge embedding processes, appears in Appendix G.

\subsubsection{Stage 1: Plotting the Course}

We said, "Yes, this is what we're going to do!"

The first stage, Plotting the Course, was about building common ground and a shared sense of direction. School leaders and change agents engaged in visioning, selecting a focus and building a coalition to support each other through the process of professional change. While most activities undertaken at this stage occurred in face-to-face settings, they provided an important foundation for the later influence of online communities. The emphasis in this stage was on how to position and enact professional change in the context of the ICT PD programme. Schools in CoPs A and $B$ also selected a specific teaching method or model (most commonly a variant of inquiry learning) to encapsulate the new way and provide a unifying focus. This gave clarity to the vision, which made it easier to gain alignment of key staff.

Table 8 summarises the activities undertaken at this stage and how they contributed to the later impact of online CoPs.

\begin{tabular}{|l|l|l|}
\hline Activity & Description & Role of online community \\
\hline Visioning & $\begin{array}{l}\text { Developing a vision for how the future } \\
\text { will be different }\end{array}$ & $\begin{array}{l}\text { Strong visioning was linked with recognition of the need for } \\
\text { change and the building of community. Online communities } \\
\text { subsequently helped reinforce the visions and develop shared } \\
\text { ownership of them. }\end{array}$ \\
\hline feclecting $\boldsymbol{a}$ & $\begin{array}{l}\text { Selecting a model or method around } \\
\text { which to base change/ embody the new } \\
\text { paradigm }\end{array}$ & $\begin{array}{l}\text { Visioning and focusing were foundational focusing steps. In the } \\
\text { next stage, facilitators selected online content and managed } \\
\text { discussions in a way that supported the focus and drove } \\
\text { change. Online communities had the most impact where there } \\
\text { was a clear focus. }\end{array}$ \\
\hline Building $\boldsymbol{a}$ & $\begin{array}{l}\text { Building an alliance to drive and support } \\
\text { change }\end{array}$ & $\begin{array}{l}\text { Principals engaged lead teachers and facilitators - change } \\
\text { agents who subsequently became core members of the online } \\
\text { community, modelling and encouraging online community } \\
\text { engagement. }\end{array}$ \\
\hline
\end{tabular}

Table 8: Stage 1 - Plotting the Course 


\subsubsection{Visioning}

The ICT PD programme required each school to develop its own vision and goals within a cluster-wide approach. During visioning, leaders established a direction, destination and purpose for their school's journey and shared this vision with key staff. Visioning began when making funding applications and continued for up to a year. School visions were based around enfolding ICT into practice in a way that improved student learning. Schools in CoPs A and B went further, deciding to use the programme to drive whole-school change:

We believed our curriculum was broken, and we wanted to change it.... We spoke to [all the cluster leaders and said] "Come on, here's ICT money, we want to change our approach. It's through ICT, but it's also through a change in pedagogy". (Principal, CoP B)

The clarity of change-based visions of leaders in these schools, coupled with the leaders' enthusiasm, helped to recruit individuals whose role would be driving change:

It was my principal's vision for the school...that plugged me in. (Lead teacher, CoP A)

School visions were less clear in CoP C, and in one case had morphed over time: A principal reported having been steered to a higher-level vision by a facilitator who had a helicopter view. The fuzziness of visions in CoP $\mathrm{C}$ was compounded by a high turnover of facilitators and lead teachers, creating a lack of continuity in key roles.

Visioning was not just about determining what the ICT PD programme would achieve, but also how this would be achieved. In particular, leaders had to consider how much compulsion they would employ in bringing staff on board. CoP C's leaders took an optin approach. Those in CoPs A and B, however, had adopted a change-based perspective, so were faced with making change a requirement. They decided to offset this with an emphasis on community building:

I saw it as a great way to try and bring the school together. (Principal, CoP A)

School leaders recognised the potential value of building community in parallel with envisaged change, but apparently did not at this stage consider using online communities as a change lever. This is interesting in light of the MOE's vision for using online communities to improve professional practice, outlined in the e-Learning Action Plan (2006a). A lack of appreciation of the potential value of online communities is a recurring theme in my findings. Visioning was a foundational focusing step for the 
online CoPs that later helped to drive the embedding of knowledge. Where schools had clear, consistent visions, facilitators were able to develop online communities to align with these visions. The clarity of vision thereby indirectly influenced the ability of the online community to drive change. Visioning was also a way of putting an organisational spin on the ICT PD programme, differentiating it from the approaches of other schools. This contributed to embedding by building school ownership.

\subsubsection{Selecting a focus}

Embedding of the new way had been most successful, and had progressed the fastest, in schools where the vision was linked with a whole-of-school focus - the introduction of a specific teaching approach, model or method. School foci in CoPs A and B included inquiry-based learning, thinking skills and student blogging. These foci can be seen as examples of boundary objects, shared objects that embody knowledge and are able to be interpreted in different ways owing to their interpretive flexibility, facilitating shared understanding (Carlile \& Rebentisch, 2003; Pawlowski \& Robey, 2004). It was possible for these schools to establish such whole-of-school foci because they were primary schools: Primary teachers teach the whole curriculum. They are general practitioners, with ample professional common ground.

The secondary schools (CoP C) did not adopt whole-of-school foci. Unlike primary teachers, secondary teachers see themselves as specialists. They teach a narrow slice of the curriculum, identifying by subject area. Knowledge is difficult to transfer across different subject silos, resulting in restricted professional common ground. Schools in CoP C had therefore taken on more restricted foci; for example, improving back-ofhouse functions, such as the administration of assessment; and developing skills in distance teaching. This was apparently a typical situation in secondary ICT PD clusters: Several interviewees commented on the difficulty in achieving broad-based professional change in secondary schools. One facilitator, who worked in both primary and secondary schools, explained that:

In primary schools, it tends to be school-wide, whole school development, and staff come across a new wave of understanding. It might be new roles for teachers, or... about the 21st century learner... Because it's whole school, it's more significant in the longer term... In the secondary area, it's very much a personal teaching [thing]...You [might] get a department who will take it on....but...it's [mainly] individuals... who can't be held back...so you harness their enthusiasm and their attitudes and ideas, and use them as champions.... 


\subsubsection{Building a coalition}

In the course of visioning, school leaders forged change coalitions. As the ICT PD programme progressed, they would draw more and more on these relationships. Building a coalition involved recruiting a facilitator and one or more lead teachers. A facilitator formed the hub of each school's change coalition. This person's job was to drive change by facilitating workshops, training lead teachers, facilitating principals' meetings and developing and facilitating the cluster's online community. Facilitators were employed on a part-time basis, and shared between schools, but their extraordinary level of influence and respect was evident in all the interviews I conducted. (One facilitator was jokingly referred to as God by a principal.) Lead teachers were the leading lights responsible for driving day-to-day change by "working structures within the school". They were given release time to devote to the ICT PD programme. Initially, much of this time was spent supporting colleagues in using ICT, but an key facet of the role was to model and drive engagement in online communities.

Building a coalition was a necessary precursor to the process of (re)aligning professional values, beliefs and understandings so they were consonant with the new way. In the first year of the ICT PD programme there was a need to build teachers' familiarity and confidence with ICT, but in the second year, the emphasis began to shift onto persuading teachers to change at a deeper level.

\subsubsection{Summary}

By the end of Plotting the Course, schools had built a change coalition with a shared vision and sense of direction. Primary schools had also selected a unifying focus - a new approach or method that would embody the new way. Knowledge of the new way was still in a generic, abstract form. The next step was to bring others on board. 


\subsubsection{Stage 2: Coming On Board}

I think I will get on board with this

During the second stage, Coming on Board, members of the change coalition worked to convince their teaching colleagues to commit to the change journey. This stage involved the building of community across two dimensions - online and offline, and two tiers - a change agent community and a practitioner community. The online dimension of these communities, based around online forums and instant messaging (IM), played a key role in facilitating Coming on Board for schools in CoPs A and B. For schools in CoP $\mathrm{C}$, the online dimension played a lesser role: forums were mainly used to record workshop outcomes, with the change agent community making strong use of IM tools. My research project aims to generate an understanding of how online communities facilitate embedding, so I have focused primarily on what occurred in the former two communities.

Facilitators built the online dimension of community by fostering social cohesion, encouraging identifying with others (being "all in the same boat") and aligning with others. They persuaded individuals to come on board by seeding reframing themes; powerful content that supported the new paradigm, reframing teaching so as to question its validity to twenty-first century students. They then challenged teachers to respond to this content. The intent was to induce discomfort, persuading teachers to see the new way as superior and to view change as inevitable.

In CoP A, the seeding of reframing themes in online forums resulted in the school practitioner communities cultivating and appropriating these themes. This was the start of the development of new, shared interpretive frameworks that would come to influence and unify thinking at school level. These new, shared views would help compensate for the discomfort induced by reframing.

As individuals accepted the challenge to come on board, they committed to change at a personal level. This happened at different times and rates for different people, and for some people it did not happen at all. These people - the reluctants - were eventually left behind as schools moved ahead on their journeys. Early boarders wielded an influence in the online communities, persuading others to come on board. The Coming on Board of individuals was a staggered affair and continued to some extent during the next two stages. 
Table 9 summarises the activities undertaken at this stage and the facilitating role of online CoPs.

\begin{tabular}{|c|c|c|}
\hline Activity & Description & Role of online community \\
\hline $\begin{array}{l}\text { Building } \\
\text { community }\end{array}$ & $\begin{array}{l}\text { Building a cohesive and mutually supportive } \\
\text { community }\end{array}$ & $\begin{array}{l}\text { Scaffolding the start-up of online forums reduced barriers } \\
\text { and increased school level buy-in to change-related } \\
\text { postings. } \\
\text { Online facilitators built social cohesion, fostering a desire } \\
\text { for identifying with others ("all in the same boat") and } \\
\text { aligning (agreeing and echoing). }\end{array}$ \\
\hline Challenging & $\begin{array}{l}\text { Challenging the validity of current practice } \\
\text { and challenging teachers to engage with } \\
\text { difficult ideas, causing discomfort and } \\
\text { tension }\end{array}$ & $\begin{array}{l}\text { Facilitators challenged teachers to relate challenging } \\
\text { content to their practice in online forums. }\end{array}$ \\
\hline $\begin{array}{l}\text { Seeding and } \\
\text { cultivating } \\
\text { reframing } \\
\text { themes }\end{array}$ & $\begin{array}{l}\text { Introducing themes and metaphors that } \\
\text { reframe (change the meaning of) existing } \\
\text { practice and varying these themes }\end{array}$ & $\begin{array}{l}\text { Online forums were a focal point for discussing thought } \\
\text { leaders' reframing themes. Cultivating these themes (by } \\
\text { adapting/varying them) amplified their impact and } \\
\text { increased CoP ownership of them, normalising reframing. } \\
\text { By doing this, the online community was "sowing the seed" } \\
\text { for change. }\end{array}$ \\
\hline $\begin{array}{l}\text { Identifying } \\
\text { and aligning } \\
\text { with others }\end{array}$ & $\begin{array}{l}\text { Empathising with others "in the same boat"; } \\
\text { recognising common issues } \\
\text { Developing ideas and/or beliefs that are } \\
\text { aligned with others/the CoP }\end{array}$ & $\begin{array}{l}\text { Views and experiences of others were made more visible } \\
\text { and gained cumulative weight in online forums. } \\
\text { Facilators encouraged alignment through moderation. } \\
\text { Teachers absorbed existing online contributions, checking } \\
\text { that they were "on track" prior to posting, and "merging" } \\
\text { others' ideas into their "thought patterns" }\end{array}$ \\
\hline Committing & $\begin{array}{l}\text { Committing to a change journey (explicitly } \\
\text { or implicitly) }\end{array}$ & $\begin{array}{l}\text { Online forums played a complementary role to workshops } \\
\text { in encouraging teachers to commit to change. Forums } \\
\text { facilitated a shift in community mindset (CoPs A \& B). } \\
\text { Some teachers attributed committing to the impact of } \\
\text { online forums - reducing their comfort levels and/or } \\
\text { promoting deeper thinking. }\end{array}$ \\
\hline
\end{tabular}

Table 9: Stage 2 - Coming on Board

\subsubsection{Building community}

Cluster facilitators worked with the lead teachers and principal of each school on building community and normalising and socialising the change agenda. Two tiers of community were developed. The first tier was the community of change agents. The second, larger, tier was each school's practitioner community, comprising its principal, lead teachers and regular teachers. These practitioners also belonged to the clusterwide online community. (Chapter 8 explores the interactions of these tiers.) 
Lead teachers began to identify as members of a change agent community, to which the facilitator and other lead teachers in their cluster also belonged. They were in particular need of support: Their role required them to lead face-to-face meetings, be proactive in online settings and, as classroom teachers, adjust their own practice. Doing all of this was a challenge. In all three clusters (CoPs A, B and C) change agents recognised their mutual needs, gradually establishing a culture of online support. I discuss this further in the next section, Setting Out.

Building the practitioner community was a more difficult undertaking because it involved cultural transformation. There was a need to counter the legacy of professional isolation arising from individuated, private approaches to teaching practice. Building a strong school practitioner community required major changes to existing relationships and communication norms. According to three principals, teachers had rarely talked about teaching and learning with their colleagues prior to entering the ICT PD programme. It is therefore unsurprising that most interviewees reported an initial lack of confidence at the prospect of contributing to online forums: The idea of espousing half-formed theoretical thoughts in front of peers had been daunting, and their reluctance had been compounded by a widespread discomfort surrounding online media: The median age of NZ teachers at the time of my study was over 50. Despite these two significant barriers, the situation was set to change quite dramatically for the schools in CoP A and, to a lesser extent, for those in CoP B.

Each cluster had an official online community area in a private space within a larger ICT PD community and resource space, hosted on a web-based platform. CoP C used this space mainly for sharing resources and making announcements. CoPs $A$ and $B$ placed an additional emphasis on building a community through facilitated forums. Community B's forums were based around discussing inter-school visits as well as professional readings, with the former drawing stronger engagement. CoP A based its forums around professional readings.

Developing strong engagement in these communities required the disruption of workplace norms in the schools in CoP A. This was managed particularly well by the cluster facilitator and the school change agents. The result was the establishment of sustained new communications norms, initially online, then, in the next stage, also in the workplace. The methods used to kick-start this transformation are outlined below. 


\subsection{Scaffolding online participation}

The facilitator and lead teachers set about breaking down the barriers to online forum engagement through a conscious strategy of scaffolding ${ }^{11}$. In CoP A, teachers initially workshopped their responses to online readings in meetings. These meetings, coordinated by the lead teachers, led to jointly authored postings that were posted to the online forums. Once online, the postings were then jointly viewed and the facilitator's (and other schools') reactions to them were discussed collectively. This scaffolding process eased the building of an online community. It had the dual benefit of gaining collective buy-in at school level and normalising engagement in online forums.

After 2-3 months, individuals were encouraged to author and post their own comments. Lead teachers used gentle humour to reward contributors and bring recalcitrant colleagues on board.

They'd put up the [web]site, and say, "Look, here's all the postage. Where's yours?" It wasn't as blunt as that....and "so-and-so was first!" ... I remember being first once... they gave me a hard time about it. That was the sort of response they had... breaking down those barriers of feeling [un]confident. And we used to share any particular comments that might have been relevant, or out of left field. We'd try not to turn it too negative... That certainly kept people going and making contributions... (Principal, CoP A)

You'd say, "Have you been on-line?", or "Have you read this?. You'd talk about it, like, "Have you read this comment about that?"... I don't think we would have [done that otherwise] (Lead Teacher, CoP A)

The scaffolding approach, combined with the requirement for everyone to participate, was successful at bringing on board teachers who were reluctant contributors in workshops:

If you were like me and weren't that confident, I could go in and see what other people were doing, and it actually made me start thinking "Oh well his thinking's right" or "that's useful", and it gave me a lot more confidence about how to...put things. Whereas before, I probably relied on what other people were thinking, and just sat back. Because it was a combined thing, whereas this time I actually had to respond, I had to put my penny's worth in... it made me more aware of what was going on. And it was good. Because everyone shared ideas, you'd get something you'd never thought of. (Teacher, CoP A)

\footnotetext{
${ }^{11}$ Scaffolding provides temporary support for parts of a task that people are having difficulty with (Bonk \& Kim, 1998)
} 


\subsection{Building social cohesion}

CoP A's facilitator was skilled at lowering barriers to online participation and fostering social cohesion. She initiated online discussions with humorous references to her own failings, acknowledged people's contributions promptly, and built constructively on their ideas while moving discussions to a higher level. A teacher described the impact of this as follows:

Nobody in our school liked going online and contributing, for several reasons: there's no face-to-face and you can't pick up tone or inferred humour, so it's a risk...Some people feel that they're talking a risk when they go online. Some people don't care. But for our school, and the nature of our staff, we did care. And so...to actually go on and comment was big for some of us. So to get instant feedback, which is what [our facilitator] did, was brilliant... She'd go "ha-ha" at the end of it, and you'd go "Oh yeah". And she'd always come back and say, "So, what you're saying is.." and, "Had you thought about this..?" There was a wonderful conversation. It was a highlight and it really broke the ice for me...

(Teacher, CoP A)

When combined with the online-offline scaffolding previously outlined, the social cohesion resulting from skilled facilitation greatly eased the process of Coming on Board. Becoming a member of this online community was like stepping onto the gangplank of a vessel about to head on a journey, amongst friends. Once on board, teachers found themselves interacting in a context where reframing of practice was the norm and where change-based themes were reinforced daily.

\subsection{Building the offline community}

Building community was also an important offline (face-to-face) activity in schools. This was occurred in part via the scaffolding approach described above, and in part via cluster-wide workshops and techie brekkies; ICT-focused breakfast meetings. The purpose was to create a sharing culture and reduce resistance to ICTs. Techie brekkies began as formal affairs, but soon developed a relaxed improvisatory character. In the next stage the offline community would undergo a greater transformation.

\subsubsection{Challenging}

Facilitators selected powerful content (themed readings and videos) to act as springboards for online and face-to-face discussions about teaching. This content aligned with, and promoted, the student-centred paradigm through diverse, persuasive arguments. Rather than being dry and academic in style, it was lively, engaging and memorable; conveying the personal voices of its authors; educational thought leaders. 
It was also change-oriented and provocative: It worked by using powerful metaphors to reframe the existing approach to teaching, making it seem antiquated, irrelevant and ineffective, while presenting the alternative vision - a student-centred IT enabled way of teaching - as superior, inevitable and overdue: Teachers became digital immigrants whose job it was to teach the digital natives (Prensky, 2005-6; CoPs A and B), while schools were accused of killing creativity, of feeding students curricular fast food (Gawith, 2004; CoP A) and of educating them from the neck up and slightly to one side (Robinson, 2005; CoP C).

The originality and impact of the persuasive content was such that it often struck a chord with the teachers, leading them to identify with the issue at stake. The content challenged people's assumptions, making them interrogate their beliefs and values:

It was challenging our beliefs about teaching... there were people that still believed that [using] computers sitting in rows... was relevant. And they were challenged by the readings. They were questioning it... and that immediately makes other people think, "Oh, what do I believe? (Principal, CoP A)

Reframing and challenging, combined with skilful facilitation and teachers' willingness to engage and reflect, had the potential to shake teachers' confidence in their approach:

[The] reading and [the facilitators'] comment made me realise that I had not come up to speed with [the way]...the children I teach learn. My god, what a shock for my self-perception! Here I was thinking that I was an OK teacher, I mean, ERO ${ }^{12}$ told me...I was outstanding. And suddenly I could clearly see that I may be one thing for ERO and something quite different for the children, and it's the children that count. (Teacher, CoP A)

\subsubsection{Seeding and cultivating reframing themes}

Facilitators then began seeding these themes more deeply. They challenged teachers with targeted questions such as, "What digital immigrant behaviour have you displayed in the last week?" (CoP A) and "What are the implications for you as a teacher?" (CoP C). Such questions forced teachers to engage more deeply with the themes, relating them to their own practice. The questions often led them to revisit their core philosophy, values and beliefs about what constituted effective teaching. A secondary school teacher commented, "Found this very poignant...it's all about what I believed in when I started working" (CoP C).

\footnotetext{
${ }^{12}$ ERO is NZ's Education Review Office. It measures school performance.
} 
By reusing the thought leaders' metaphors in questions, facilitators encouraged forum participants to adopt the same metaphors ("In what ways are you adapting your teaching for the digital natives?'). As teachers reflected on these questions, they began cultivating the new themes and metaphors. They invented diverse variants of them and wove these into their responses in online forums. This sometimes had a snowballing effect: Figures 23 and 24 show the variants of the digital immigrant metaphor (Prensky, 2001) that were used by teachers in an online forum in CoP A over a three week period. Figure 23 illustrates selected variants, while figure 24 shows how thematic variants proliferated in two main families (adapting themes/phrases and immigrant themes/phrases) as the contributors aligned with each other. (Prensky's original metaphors are asterisked).

This brief but intensive period of metaphor cultivation, combined with incessant repetition of the source themes, facilitated the embedding of these reframing metaphors in several ways: (a) through the sheer cumulative impact of repetition, (b) through the enrichment of meaning that resulted from participants' cultivation and extension of the source themes, and (c) through growing ownership (appropriation) as the themes were contextualised in a way that made them more relevant to the community and its individuals. Not only the language that was being embedded, but also its meaning.

\begin{tabular}{|l|l|}
\hline $16 / 6$ & "What immigrant behaviour have you displayed in the past week?" (Facilitator) \\
\hline $17 / 5$ & "...that is my digital immigrant stance" \\
\hline $22 / 5$ & "I believe...respect for the heritage of our "Digital Immigrants" has a part to play" \\
\hline $22 / 5$ & "I cold see a tiny glimmer of hope that my immigrant status was changing!" \\
\hline $22 / 5$ & "... I need to stay a little longer in the refugee centre" \\
\hline $23 / 5$ & "I am in between two generations of digitals" \\
\hline $24 / 5$ & "I am...applying for residency... but will always be a bit of a foreigner." \\
\hline $24 / 5$ & "Thank you all those would-be natives out there for pulling me along..." \\
\hline $25 / 5$ & "Tools will constantly change making the...natives of today tomorrow's immigrants! \\
\hline $25 / 5$ & "I have two teenagers who believe that I am a digital dinosaur..." \\
\hline $26 / 5$ & "I am stuck in an immigrant world" \\
\hline $31 / 5$ & "...us old immigrants" \\
\hline $1 / 6$ & "I have...been a reluctant immigrant but as I come to be more digital savvy..." \\
\hline $6 / 6$ & "(I) have definitely adapted to my environment..." \\
\hline $24 / 6$ & "Life as an illegal alien" \\
\hline
\end{tabular}

Figure 23: Cultivating the digital immigrant theme. Online forum, CoP A (added bolding). 


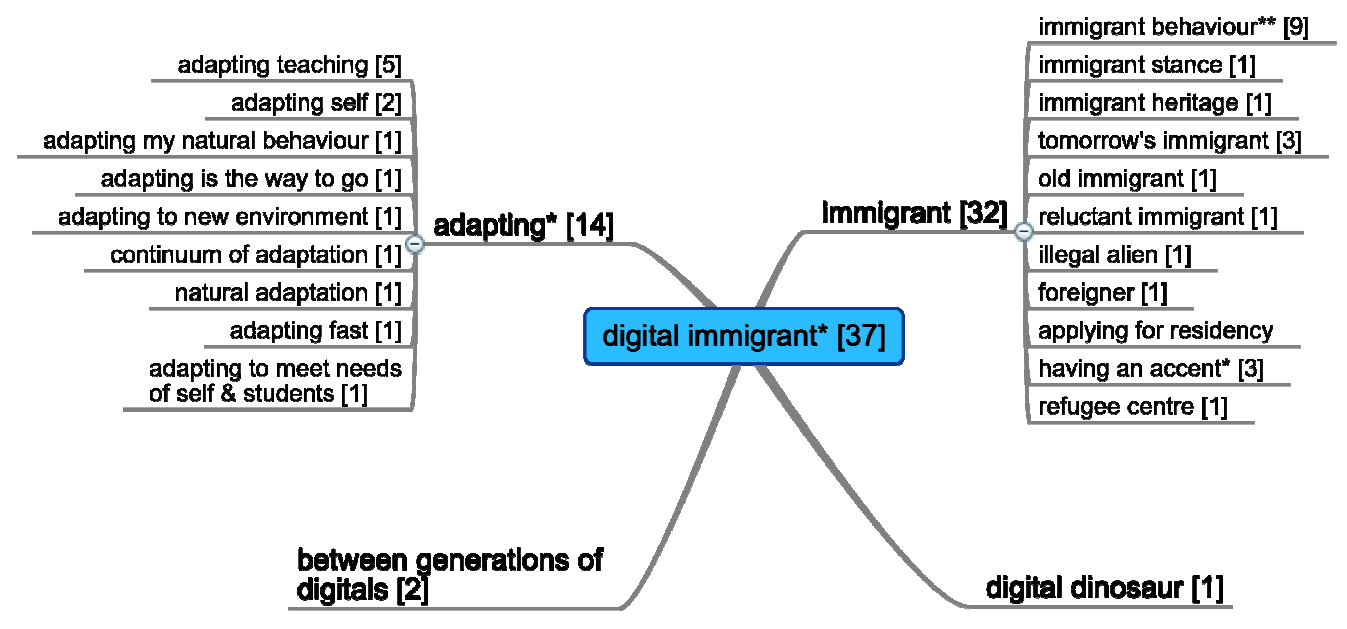

Figure 24: Cultivation of a theme from Prensky (2001) in online forum, showing the alignment of thematic variants within two families of metaphors (CoP A)

Members of CoP A cultivated metaphors in such a way that they remained true to the intent of the source; reframing teaching to question its relevance. This was exemplified by the coining of the humorous, yet pejorative, terms digital dinosaur and illegal alien.

Even when responses defended an aspect of the status quo, there was an implied acceptance of the reframed perspective, evidenced by the authors' willingness to continue cultivating metaphors:

I believe that respect for the heritage of our "digital immigrants" has a part to play in the overall scheme of things. But then maybe I need to stay a little longer in the refugee centre and learn yet more to be able to keep up in this ever-changing world. (Teacher, CoP A, added bolding)

Regardless of the exact terms used, teachers had been repositioned as immigrants; people who were poorly integrated into the digital culture that was part of students' lives. The impact of such discussions was significant. One principal noted:

I think the online dimension was sowing the seed for the school....to develop other things happening within the school, so that we could churn it over, I suppose; come to where we've got to.

This seed analogy supports Thorpe's (2003a) suggestion that online CoPs may provide generative value in the context of change: "If the community has to do something new, or developed added value from working online, that may be the kernel of what motivates them to alter already well-developed modes of working" (p.31). The reframing themes that had been seeded and cultivated during Coming On Board would begin to flourish during the next stage, as they were taken into informal workplace conversations, further appropriated, and sustained by the community. 


\subsubsection{Identifying and aligning with others}

As cohesive social norms developed in the online community, teachers often identified with each other ("Like [Penny] I teach very young children...'). They also agreed with, and echoed, each other's comments: "I also agree [with Allan that] today's native will be tomorrow's immigrants...". This was a self-reinforcing process: By iteratively aligning with each other and with the change themes - echoing and cultivating metaphors in a way that embedded their meaning - members persuaded themselves, and each other, to come on board and commit to the change journey. The impact of this online persuasion in was particularly strong in CoP A, where it can be attributed to a combination of factors: the high level of teacher engagement, the facilitator's skills and the school leaders' support and willingness to participate alongside their staff: "As a leader I thought, "I'd better do it". I can't just say, "You lot, do this!"

The asynchronous nature of forums allowed teachers to participate when and where suited them. This allowed them time to review others' comments, absorb their cumulative impact, and ensure their own responses were aligned appropriately.

People could do it at home, they could go on any time of day or night, and see what other people had put. And it wasn't just us, it was sixtyodd teachers sitting there, making comments. (Lead teacher, CoP A)

When I respond it [is]... after reading others so I know if I am on the right track... after thinking about the article, about what others have written and then when I have time [to work out] what I think. (Teacher, CoP A)

The combined impact of the facilitator's moderation and the community's opinions led not only to alignment, but also to a deepening of individual thinking.

As the readings went on and the moderations went on, you thought more deeply...rather than just you having your opinion, you were sort of forced to think about the other person's opinion, and then maybe then other ideas would come up as a result of that. So I think that you, certainly you broadened how you thought, and also perhaps reinforced; "Yes, this is the reason why I think like this". (Lead teacher, CoP A).

Through the cultivation of themes, the online community was developing strong ownership of the change-related themes.

\subsubsection{Committing}

The objective of Coming on Board was to gain collective and individual commitment to professional change; not merely a willingness to use ICT or a receptiveness to the new way, but a genuine readiness to engage in action. In CoPs A and $\mathrm{B}$, the combined 
efforts of persuading and building community resulted in a shift in the collective mindset in schools to the point where there was a readiness to embrace change. Committing at this level meant being willing to step out of professional comfort zones and to accept disruption to existing routines:

[This was] a very tidy, organised school. I've sort of tipped that upside down, and it's a huge change.... but the teachers have embraced it absolutely willingly... (Principal, CoP B)

In CoP A, this shift in mindset was embodied in the community's online alignment with, and growing ownership of, change-based themes, their implicit acceptance of reframing, and their newfound solidarity: "We're all in the same boat".

At an individual level, Coming on Board was a somewhat different affair. One facilitator said:

It's a deeply personal decision. And ninety percent of the time, it matters little what's happening around the teacher. The teacher really has to...be convinced in some way, themselves, before they make any big shift. (Facilitator, CoP A)

In some cases, the decision to commit to change was triggered by a desire for greater clarity in the understanding of their own teaching:

That link between theory and practice, it was never there before, but through those [online] discussions, I was able to make that link, and that sort of challenged me to decide to understand how I operated in the classroom. (Teacher, CoP A)

In such cases, committing was about resolving uncertainty. The decision was promoted by the nature of online discussions; specifically the way in which they challenged teachers to link theoretical readings with classroom practice.

In a few cases, teachers stated that their decision to change had been made quite suddenly:

It was almost like being struck by a bolt of lightening: "My God, things have changed! l'd better get my head out of the sand." (Teacher, CoP C)

However, it seems likely that hindsight made people's decisions to commit appear more rapid than they were in reality: At a participant check workshop, two facilitators noted that in their experience, bolt of lightening decisions were rare ${ }^{13}$. In their opinion,

\footnotetext{
${ }^{13}$ My contention about hindsight is supported by research by Wagenaar that suggests people have a poor ability to reconstruct the chronology of past events (Wagenaar, 1986).
} 
teachers most often committed to change after an episode of temporary immersion, such as a workshop, where they were removed from day-to-day routines. In relationship to such immersion episodes, online communities may have had some kind of complementary and/or reinforcing effect. Perhaps online discussions helped to cultivate a frame of mind in which individuals were predisposed to commit to change, and/or perhaps they helped individuals consolidate and clarify their thinking after workshops. Many teachers spoke of using IM to discuss workshops with colleagues after the event:

If we'd had a visiting speaker...we talked a bit about that [on Skype].... bouncing ideas off each other...saying, "I'm going to try this in the classroom", or skyping someone and saying, "I tried this, from that today", and telling them how it went. (Teacher, CoP B)

The asynchronous nature of forums provided for a complementary, more personal, kind of immersion experience. Most teachers engaged with forums in the evenings, when they were away from classroom routines and had the time and headspace to engage more deeply and at their own pace. One principal noted the superiority of this kind of immersive experience for deepening deeper understanding and ownership of ideas:

Ironically, for a lot of teachers, being in a group situation is quite threatening and they're not prepared to share their ideas....[but] online learning is great, because you can sit there, you can read, digest in your own time, then reply, and it's far less threatening... I myself enjoy it, because you get to see a whole range of comments, and then merge that into your own thought pattern, in your own time. (CoP A)

It is possible that, in relationship to workshops, online discussions may have acted as a kind of tipping point (Gladwell, 2000), tipping individuals towards making a commitment in relationship to that forum's content:

Jamie McKenzie struck a chord with me by mentioning "the cheapening and dilution of the authenticity of experience".. Who would want to be guilty of that? I am promising myself to use discretion and discernment when using clipart in future" (Teacher, forum, CoP A)

Online interactions probably impacted in a cumulative way over time. The forum discussions can be seen as building on and reinforcing face-to-face experiences, creating a timeless zone that was (a) suitable for reflecting on the new knowledge, (b) flexible enough to allow reflection at the best time and (b) strongly persuasive, with the collective voices of the facilitator and peers advocating for commitment to the new way. 
For some principals and teachers, coming on board was seen as a necessary way of keeping up to date. One principal used the analogy of a dentist to convey this idea:

I liken it to a doctor or a dentist. I say, "Do you want to go to a dentist who is still doing things the same as they did twenty years ago?" (Principal, CoP A)

Some teachers came on board out of the simple realisation that if they didn't, they would be left behind.

If you don't jump on and change, you just get left behind. So you just have to. That's what I tell my husband. We have to jump on that bandwagon. (Teacher, CoP A)

\subsubsection{Staggered boarding}

Teachers neither came on board at once, nor boarded with the same speed and enthusiasm. Those who boarded first were the change agents (lead teachers and principals) and other enthusiasts. To extend Prensky's metaphor, they were the first wave of immigrants. They paved the way for subsequent travellers by persuading them that it was safe to come on board. The online community provided a suitable means for doing this.

Lead teachers were in the minority at their own school, but had a greater level of comfort than their colleagues online. This meant they could readily combine their persuasive weight with that of other lead teachers, influencing the direction of online discussions. In the following example, a lead teacher incites their colleagues to commit to action:

The Nike motto of "just do it!" is the way that I have found to be most useful. You can theorise and justify, but if you believe that learning "new stuff" is easier than "learning new ways to do old stuff" there is really no other way. (Lead Teacher, Forum, CoP A)

As is typical with any change process, each school had individuals who were opposed to change. These people were left behind as the larger community moved ahead on their journey. The intention was to collect them at a later stage, once embedding had occurred at school level.

The Coming on Board stage did not have a clear end point. It continued to some degree during the following two stages as more individuals were persuaded to come on 
board. The late boarding of such individuals was compensated for to some degree by the final Settling stage, in which change was stopped, giving them catch-up time.

\subsubsection{Summary}

Coming on Board was the critical first step in professional change. It was strongly facilitated by the building of new norms and group constructs in the online community. During this stage, change agents gained an online environment in which they could interact and identify with each other. Online forums acted as a change lever for CoPs, as facilitators built a culture of social cohesion in which it was safe to discuss professional knowledge. This facilitated the seeding and mutual cultivation of powerful, persuasive reframing themes. The new way became bound up with constructs of professional identity that challenged the status quo, such as the teacher as digital immigrant. As the new themes took root and individuals reflected on them, the collective mood changed, and individuals began to commit to change. 


\subsubsection{Stage 3: Setting Out}

What a great journey we are all on!

In the Setting Out stage, schools and teachers began to engage more directly with the new way through practical action. They began piloting different methods and models with the result that new knowledge became more distributed across the cluster. This increased the value of online collaboration. There was a corresponding increase in the frequency of online interaction and an expansion in the range of online tools used. Activities aimed at building community continued, with facilitators fostering social cohesion while priming change agents to sustain discussions. As the CoP developed, online discussions of professional knowledge were normalised. Once established, this norm crossed into the informal workplace, creating a new culture of knowledge sharing. This, in turn, enriched the quality of online interactions, creating a virtuous online-offline communication cycle. Online forum reframing themes also crossed over into the workplace and were appropriated and normalised by the offline community.

Embedding of the new way began in earnest with a shift to going deeper - engaging more deeply with its meaning and implications. Facilitators used drawing out and challenging to shift online discussions to a higher level. Individuals undertook piggybacking - building on the ideas of others, as expressed in forums, to enrich and align their thinking. Teachers reflected on their practice, comparing it with the theory of the new way and checking its fit. They started problematising - identifying specific issues for resolution. Iterative adaptations would be necessary to gain a better fit. The matching up theory and practice would become more important during the next stage.

Online forums made the thoughts and practices of colleagues more visible, facilitating the identification of like-minded individuals. This led to the establishment of buddy partnerships, which were sustained through side-conversations using $\mathrm{IM}$, texting and email. In all three clusters, the change agent sub-community developed a vibrant culture of synchronous online interaction, based around day and night-time use of IM tools (Skype or ichat). A norm of being online developed, facilitating just-in-time support, sharing of ideas and interrogation of problems in invisible side-conversations. Change agents began to identify with and follow online influencers - respected practitioners and thinkers who published edublogs.

Table 10 outlines the activities undertaken at this stage and the role of online CoPs. 


\begin{tabular}{|c|c|c|}
\hline Activity & Description & Role of online community \\
\hline Piloting & $\begin{array}{l}\text { Trying out a borrowed model or } \\
\text { method over a period of time } \\
\text { and evaluating its success }\end{array}$ & $\begin{array}{l}\text { Piloting provided a shared focus that helped unite the online community. As } \\
\text { schools piloted different models, knowledge became more concrete, specialised } \\
\text { and distributed, increasing the value of the online CoP as a knowledge source. } \\
\text { Individuals shared and compared emerging knowledge via forums and IM. }\end{array}$ \\
\hline $\begin{array}{l}\text { Building } \\
\text { community }\end{array}$ & $\begin{array}{l}\text { Building a cohesive and mutually } \\
\text { supportive community }\end{array}$ & $\begin{array}{l}\text { Facilitators continued to build cohesion and primed individuals to sustain and } \\
\text { enrich community interaction. } \\
\text { Interaction in forums normalised the discussion of professional knowledge. } \\
\text { Once established, this norm crossed into the workplace, creating a new culture } \\
\text { of knowledge sharing. This, in turn, enriched online interactions, creating a } \\
\text { virtuous cycle. } \\
\text { Buddying: Online interactions made the thoughts and practices of colleagues } \\
\text { more visible, facilitating the identification of like-minded individuals. This led to } \\
\text { the establishment of buddy partnerships, which were sustained in side- } \\
\text { conversations using IM and email. } \\
\text { Building the change agent community: Change agents developed a vibrant } \\
\text { online sub-community based around day and night-time use of IM. A norm of } \\
\text { being almost always online enabled just-in-time support and sharing of ideas/ } \\
\text { and problems outside the larger, visible, forum-based community. }\end{array}$ \\
\hline $\begin{array}{l}\text { Cultivating } \\
\text { reframing } \\
\text { themes }\end{array}$ & See table 9 & $\begin{array}{l}\text { Teachers continued cultivating reframing themes in forums. These began to } \\
\text { cross into face-to-face workplace discussions. }\end{array}$ \\
\hline $\begin{array}{l}\text { Going } \\
\text { deeper }\end{array}$ & $\begin{array}{l}\text { Engaging more deeply with the } \\
\text { new knowledge and its } \\
\text { implications }\end{array}$ & $\begin{array}{l}\text { The facilitator used drawing out and challenging to shift discussions to a higher } \\
\text { level, aligning them with the new paradigm. This encouraged problematising; } \\
\text { articulating specific issues to do with applying the new way. } \\
\text { A culture of nightly IM amongst change agents facilitated going deeper into } \\
\text { issues via side-conversations that did not fit forum topics. } \\
\text { Piggybacking - building on the ideas of others (as made visible in forums) } \\
\text { facilitated the enrichment and alignment of thinking. }\end{array}$ \\
\hline $\begin{array}{l}\text { Matching up } \\
\text { theory and } \\
\text { practice }\end{array}$ & $\begin{array}{l}\text { Comparing new knowledge and } \\
\text { existing practice and making } \\
\text { adjustments }\end{array}$ & $\begin{array}{l}\text { The online community culture encouraged individuals to identify and share } \\
\text { aspects of their practice that fitted/did not fit, the new way. This was the start } \\
\text { of an ongoing process of adapting through iterative aligning of practice with } \\
\text { theory. }\end{array}$ \\
\hline $\begin{array}{l}\text { Identifying } \\
\text { influencers }\end{array}$ & $\begin{array}{l}\text { Selecting influential people to } \\
\text { follow (respected practitioners } \\
\text { and thinkers) }\end{array}$ & $\begin{array}{l}\text { Some individuals began to identify and follow influencers - practitioners and } \\
\text { thinkers - who kept blogs. Common membership with CoP E made this easy. }\end{array}$ \\
\hline
\end{tabular}

Table 10: Stage 3 - Setting Out 


\subsubsection{Piloting}

Schools began piloting student-centred learning approaches, models and methods that fitted their chosen focus. Schools in CoPs A and B piloted different inquiry learning models, while those in Community $B$ also piloted models for developing thinking skills. Linked with these broad approaches, teachers piloted methods for the use of ICT, including WebQuests ${ }^{14}$, student blogging, the use of digital drawing packages and the use of IM for student peer-to-peer mentoring ${ }^{15}$. Their aim was to gain a hands-on understanding of how to use ICT to foster the new way. One teacher noted,

We [had to] find an inquiry process, and actually do it ourselves, and put [ICT] into [our] programme with the theory... we were actually going in and doing it, and finding that we could make a difference.

Figure 26 explores the relationship between the new way and the learning models, methods, and technology, in terms of professional knowledge. Effective teaching is shown at the top level. Knowledge of the new way sits below this, and influences it.

The new way comprises practical and theoretical dimensions. Learning models (e.g., inquiry learning) are the boundary objects (Pawlowski et al., 2000) used to aid understanding and delivery of the new way. They cannot be delivered without complementary practical methods (e.g., knowing how to make WebQuests work).

\begin{tabular}{|cl|}
\hline Effective Teaching & $\begin{array}{l}\text { - Intentions } \\
\text {-Actions \& outcomes } \\
\text {-Adaptations }\end{array}$ \\
\hline The New Way & $\begin{array}{l}\text { : Theory \& rationale } \\
\text { - Practical application \& benefits }\end{array}$ \\
\hline Models & $\begin{array}{l}\text { - Inquiry learning } \\
\text { - Thinking tools... }\end{array}$ \\
\hline Methods & $\begin{array}{l}\text { - Webquests, Skype mentoring } \\
\text {-Student blogging... }\end{array}$ \\
\hline Technology & • How to use Ict \\
\hline
\end{tabular}

Figure 25: Dimensions of knowledge being embedded

\footnotetext{
${ }^{14} \mathrm{~A}$ WebQuest is an inquiry-based activity in which students gather information from websites pre-selected by the teacher (Dodge, 1995)

${ }^{15}$ In schools in CoP C, piloting occurred at the individual level only, but plans were underway for department-level piloting.
} 
Likewise, ICT-based methods cannot be applied without technical knowledge about the use of ICT (e.g., how to make an electronic whiteboard work). Schools needed to embed all these types of knowledge simultaneously, while prioritising the higher level constructs. (This model is intended to help the reader understand the constructs schools were working with and how they interrelate. The reality was that professional knowledge was a more complex amalgam.)

Piloting was an internally focused activity, with each school testing out a model, or series of models, with the aim of finding the one that suited it best. This would increase the common ground at school level, creating the breadth of shared knowledge that was necessary for embedding. As one facilitator commented:

I believe it's really cool that you use this model as a whole school. To quote one of your teachers, 'We all sing from the same song sheet'. You're creating a common language and a school culture that really helps embed [it] into place across the school. (Forum, CoP A)

This localised approach meant that as knowledge of the new way began to develop, in the form of emerging understandings and practical learning, it became more distributed amongst the schools in each community. This increased the value of collaborating online.

[It was greatl] looking at what other schools had been doing... the lead teachers would talk about things that they had, how to use it, how it worked, ideas about how to use it, lots of things. People would send us examples that they'd actually used - it was really good. (Lead teacher, CoP A)

At this stage, the new way was in a fluid state, with schools and individuals exploring their styles, preferences and 'takes' on student-centred learning. This fluidity, plus the shared focus that united each community, meant that the new knowledge was highly shareable. The fact that was actually possible to share it was a novelty: In the schools in CoPs A and B, the new visibility to others' thinking and actions, combined with a new culture in which inter-professional communication was safe, made it not just possible, but also highly desirable to share knowledge. This was the result of the successful building of community.

\subsubsection{Building community}

During Setting Out, community-building activities continued and diversified. Facilitators started priming change agents and other individuals to take a more active role in forums. This was a tactical form of persuading that aimed to reduce the community's 
reliance on the facilitator, transferring the sustaining of dialogue those who would become local experts:

I primed up one or two others... and the conversations were sustained by other people, rather than me, and they were asking questions of each other, and posing questions, and commenting... there was real depth in the conversations in the community at that point. (CoP A)

In CoPs $A$ and $B$, the increased frequency and quality of online interactions, together with the new culture of open professional dialogue, had two important outcomes. The first was the normalising of discussions about professional knowledge. The second was a new level of visibility to colleagues' thoughts and practices. In combination, these things triggered a significant transformation in workplace culture. Prior to embarking on the ICT PD programme, the schools in each cluster had had little knowledge of what the others were doing. Within schools, teachers had had little access to their colleagues' thinking, and little knowledge of events happening in each other's classrooms. In CoPs A and B, all of this changed, as the gains made in the online community were unexpectedly transferred to the workplace.

In CoP B, an open day was followed by online forum discussions emphasising the value of colleagues' methods. The positive nature of these interactions increased teachers' sense of safety to the point where they began to spontaneously share knowledge:

The teachers have started sharing. They've opened up their classrooms, so we walk the talk; we go visit each other; share our practice.

(Principal, CoP B)

In the four schools in CoP A there was a more dramatic transformation. To the surprise of school principals, the culture of professional online discussion crossed over into the informal workplace agenda. The online themes started to permeate face-to-face discussions, dramatically changing the tenor of workplace interactions. The impact of this change is evident in excerpts from interviews with two principals. In the first example, the principal attributes the dramatic change in workplace culture to the challenging nature of discussions about the new paradigm.

It was a stunning online community, in that the on-line discussion happened online, but then it happened within the staff-room that day...People would come in... and say, "No, I actually agree with that comment," and it created this wonderful... atmosphere of discussion about what learning and teaching was. And we weren't, by the by, discussing rubbish, and who wasn't on duty and so on, but the majority of the discussion in the staff-room was [about] what was happening on- 
line. So, while I found the on-line discussion interesting, I was, as principal, more fascinated by the flow-on effects within our staff-room.... And the reason, I believe, for that was that what we were discussing and reading about was sort of controversial, new; was challenging the teachers, and their beliefs about what teaching was, and what learning actually was. (CoP A)

The second principal attributed the change in culture to teachers' desire for alignment:

... the whole conversation in the staffroom changed from talking about the things that weren't about learning to talking about stuff that was about effective teaching and learning. It was whole change, and it happened quite quickly..."

[l:] What were they talking about before?

You know, what colour the shed was going to be painted ... Instead of just talking about kids' behaviour, or what they had for lunch, or whether they had good lunches, they started talking about effective ways of teaching....It was brilliant and I couldn't get over it. Suddenly there were teachers talking teaching language!

[l:] And you feel it was the online environment that [triggered that]?

That was part of it, because it made them think about what they'd been reading and discuss it with someone else. Before they'd put it on[line] for everyone else, they needed to talk with their mates about it. (CoP A)

There is little doubt that that the careful scaffolding of online engagement had played a key part in this school's cultural transformation. Even after the formalised scaffolding in schools had ended, many teachers noted that they saw a need to test out their thinking with colleagues prior to exposing it online.

I would read [the forum comments, then] write some notes down on post-it notes with some questions and things and I would just about always go and talk to somebody else about what they thought about it. So l'd still have that interaction with somebody else, because I like putting [out] thoughts and ideas... because it formalises what's happening up here [in my head], before I go and post things on the Internet." (Teacher, CoP A)

I seem to go through a rather slow process. First I read the reading, then I look at the question. Next I spend time looking at other people's responses, once, twice or even more! I might talk about it with other colleagues...This process could go on for a week. During this time I am reflecting and then I finally get round to posting." (Teacher, CoP A)

The resulting workplace discussions enriched the quality of subsequent online interactions, which in turn fostered new offline discussions. This resulted in a virtuous communication cycle and a degree of fluidity between the online and offline dimensions of community that continued until the Anchoring stage. 


\subsection{Buddying}

The online community was, by now, creating an unprecedented level of visibility to the emerging practices and thoughts of other teachers. Through participating in and/or reading online discussions, individuals discovered others with similar interests and like thinking:

There were things that, even within [my] school, one of the teachers had been using, and I didn't know she was in the school doing that.... I could actually walk over to the classroom and say, "Oh, can you show me how to do this?" It was quite good...It encouraged that talk within the school, where you've read something....and you could say, "Oh, I've read your comment about ra-ra-ra,", and actually talk to the person, rather than doing it on-line. (Teacher, CoP A)

This led to a number of instances of buddying - sustained, productive partnerships through which like-minded teachers helped each other with their problems. Buddying between teachers within schools was sustained primarily through face-to-face interactions, while buddying across schools was more dependent on online communication via IM tools, in so-called side-conversations: discussions outside the larger forum-based community that were off the visible record.

Buddying is exhibited in the example below, where two change agents in CoP B, "Steve" and "Jess", engage in a night-time IM exchange. They firstly engage in aligning, identifying with their facilitator's efforts to bring negatives on board and expressing solidarity over the difficulties of being at the coalface. Jess then shares information about a change-focused reading, indicating a shared concern, and introduces two new metaphors - sandpit time and cross-pollination. This turns the conversation to a positive note, building owning of shared, change-focused resources. The metaphors can be seen as persuading devices, further legitimising change. Crosspollination emphasises the value of sharing, reinforcing community spirit.

Steve: [Our facilitator] was good value today, she gained an appreciation of what its like to work with some of our more negative ICT luddites

Jess: I have found a reading about getting the negatives on board...it's about nudge and nurture... haven't read it but it's meant to be good.

Jess: It is hard work at times, i think the facilitators forget and they also forget the other stuff in out lives.

Steve: oh really, flick me the details would love to read it Jess: Today i discovered the latest terms are sandpit time and crosspollination!!

Steve: sandpit time? 
Jess: I could send it via skype as a file if i'm clever enough...bear with me and i'll find it online and try!!

Peter: ok

Jess: Sandpit time...that just means time to play with the stuff...ict tools and what they can and can't do!

Jess: cross-pollination is when you network with others and share ideas! Steve: oh cool, i like that

Over time, side-conversations like the one above came to play a vital role in supporting individuals as they developed understanding of the new way.

\subsection{Building the change agent community}

The above conversation illustrates how, during Setting Out, change agents recognised a need for regular, free and frank communication amongst themselves. They began supplementing their contributions to forums with invisible, synchronous peer-to-peer activity. IM tools (ichat or Skype) became the communication method of choice for these informal side-conversations, providing a means of interacting quickly and invisibly outside the larger community. A norm developed whereby the change agents made themselves available for each other outside of working hours:

I always turn Skype on so that people know that I'm there... I don't always initiate the conversation, but if somebody pops up, then I'll have a conversation. (Principal, CoP B)

What had previously been solo evening planning time was transformed into a time for fluid community activity. This occurred amongst change agents in all three clusters. IM provided a means through which lead teachers could identify with each other, express doubts, offer practical tips and comment on forum topics in a safe environment. This created a powerful sense of solidarity.

\subsubsection{Cultivating and sustaining themes}

During Coming on Board, the cultivating of reframing themes was carried over into the workplace, with the new communication norms. In one school in CoP A, the phrase "I'm a digital immigrant" was strongly appropriated, where it was used as a uniting theme or touchstone, fostering good-humoured collegial alignment:

That's something that definitely stuck in my mind, that I'm definitely an immigrant, and not a native [laughing]... That helped me to understand why I do a lot of things that I do... and relate it to other people, in the way they see things, or the way they use technology in their classroom, or don't...

[I] So what does that mean in terms of your actual teaching? 
What it means is, I'm more aware of it, so I make more of an effort with the children in the classroom than perhaps I did before, but also, I guess I use it as an excuse... (Teacher, CoP A)

The digital immigrant metaphor had, by now, acquired a richer, context specific meaning unique to this community. As a reframing metaphor it had initially created dissonance - undermining teachers' comfort levels by suggesting their current practice was inadequate. Now, as a catchphrase, it provided a form of stability in the face of change. It united the community and fostered alignment, providing a shared framing device for stories and a touchstone against which to measure progress. This normalised the difficulties of the professional change journey, reinforced the need for the journey, and helped individuals recognise the progress they were making.

It's very interesting...digital immigrants and the digital natives, those words came up all the time, in general conversation... On-line it was interesting... Some people that said that they weren't even a digital immigrant; they were something before that. But seeing the changes [they had made] people would say, "I'm a digital immigrant, but now I'm doing this!" (Lead teacher, CoP A)

\subsubsection{Going deeper}

Embedding of the new knowledge began with a shift to going deeper-engaging with the philosophy and beliefs underpinning the new way, its personal implications and its practical applications. Forum discussions became more interactive, practically focused (linked with piloting) and extended. (As one participant exclaimed, "The more you go into a topic, the more complex it becomes.") Teachers in CoP A spoke of having gained increasing value from forums as the discussions helped them "flesh out" and clarify their thinking and raise it to a higher level. Teachers began problematising, identifying specific issues that they faced and needed to resolve. (For example, several junior teachers anticipated problems in applying the new way with young children: "I don't feel that five and six year olds have the prior knowledge or life experience to engage in original thinking on an individual basis. ') Problematising was triggered by the online discussions, although the issues were not necessarily shared online. One facilitator described this process as the being the start of embedding:

[You start] by discussing... putting out the ideas, and then you start the [real] thinking.... "How is this going to affect me and my practice, and what does this mean for my kids?"... that, to my way of thinking, is the start of the embedding.

The following activities contributed to going deeper: drawing out, challenging, piggybacking, problematising, having side-conversations and indentifying influencers. 


\subsection{Drawing out}

In CoPs A and B, the facilitators and primed change agents started drawing out participants in forums, asking focused questions that required a deeper level of engagement. These questions typically related to the practical use of learning models:

This is a new organiser for me and I would be interested in your feedback and thoughts as to how the children are finding this organiser... I can see how you would use [it] to prioritise... I would be interested to learn about the responses the pairs and small groups give... How do you [do this?] (Facilitator, CoP A)

In CoP B, drawing out was used to elicit constructive feedback following a field trip in which teachers had seen each other using different methods:

I was impressed with the independence that all groups showed... I wonder how much of a challenge it is to get children to work independently...I wonder if [they] were independent workers in Term One? (Principal, CoP B)

Teachers were well aware of the purpose of these facilitation tactics:

Rather than just surface thinking, she was trying to get us to do some higher order thinking, I'm sure of it. (Lead teacher, CoP A)

Underlying the drawing out tactic was a concern with building the informational value of the community, while reducing demands on individuals. In the following example, the facilitator asks participants to provide one example of an idea they have used from another school.

Which ideas? How did you use them?... There are so many ideas out there. Can we each elaborate on just one in this online environment. I saw ...the students at [school name] make a digital story. The children were in pairs, They introduced each other and then outlined what their learning goal for the year was. This had been burnt onto CD. It will be interesting to revisit the $C D$ mid-year and ask how are they going about achieving the goals they had set. (CoP B)

When a participant fell into line with this suggestion, the facilitator rewarded the contributor with a photo that matched their interest: "Here's two photos of scaffolding for blogs... Hope they help" (see figure 26).

Drawing out was more successful in CoP A's forums than in CoP B's in achieving a deeper level of thinking. This was no doubt partly due to the requirement for teachers in CoP A's schools to contribute online. A further explanation may lie in CoP B's online communication culture. This cluster's first facilitator had used a blog, rather than 
forums, as the focal point for professional discussions. This had resulted in a culture of online engagement that was antithetical to the building of value in forums. The use of blogs is associated with a perception of value as lying in distributed, rather than centralised, repositories. Schools in CoP B had developed a culture whereby change agents and others communicated their responses to the facilitator via sideconversations outside the initiating discussion space. As the former facilitator explained:

Sometimes things l've put on my blog will prompt real conversations rather than virtual ones with people, and sometimes people will leave comments, and I'll e-mail things to them they've talked about, or asked about. So there's a number of different ways those conversations happen.... (Facilitator, CoP B)

It appeared that the leaders of CoP B had not considered the additional benefits that might have arisen from fostering more intensive participation in online forums.

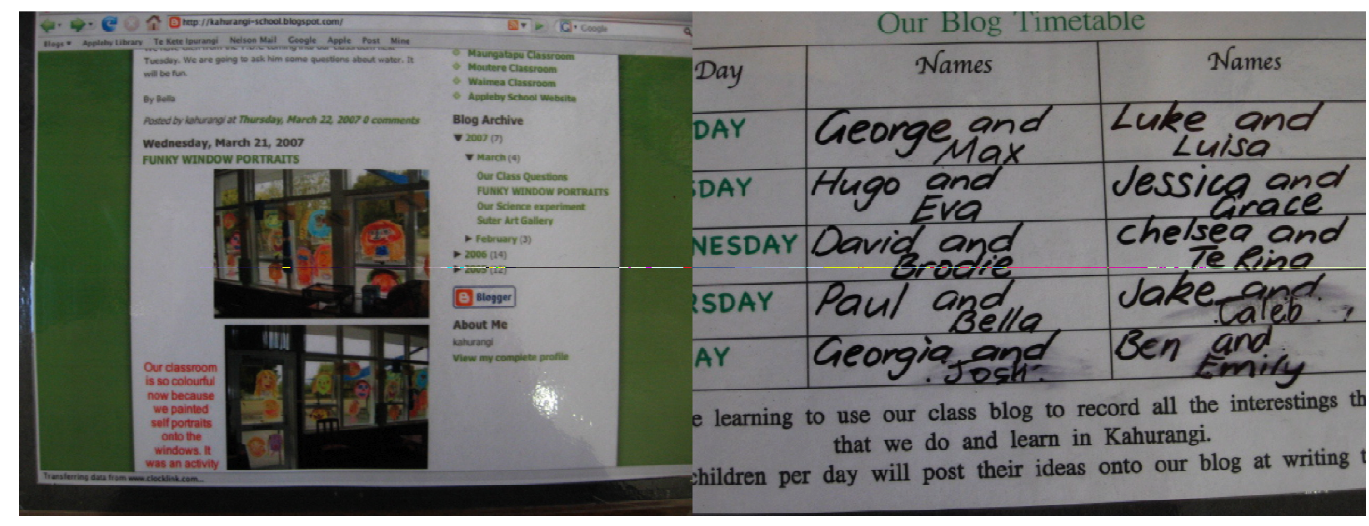

Figure 26: Photos posted in a forum by the facilitator to reward a contributor

\subsection{Having side-conversations}

I have previously mentioned side-conversations. These were valuable for going deeper when forum discussions went off-agenda or became risky. In some instances, a conversation that began in a forum was shifted into a series of IM-based sideconversations between buddies. (This is exemplified in an interchange about WebQuests in section 6.4.4.3) In other cases there was a more sustained counterpointing between IM and forums, with the former being used to test out halfformed ideas (seeking alignment) and the latter for expounding a fully formed point of view. IM was then once again used to go deeper, justifying the opinion that had been posted, with the intent of influencing forthcoming forum responses. In the following IM 
excerpt, a principal aims to influence a colleague's online forum comments so as to secure cohesion (alignment):

[Lead Teacher]: Just read your reply... You make some good points to encourage the conversation to go further

[Principal]: Thanks......there are so many issues and aspects but let's...acknowledge all the good things that are being done out there by our teachers...let's acknowledge that they are prepared to make the changes!

Teachers also picked up on IM conversations in the workplace or in cluster workshops:

'[Ann] said to [Peter], "The conversation we had on-line the other night, about the Prensky article, digital natives/digital immigrants, this is what I was meaning..."'(CoP B)

As thinking moved to a deeper level, conversations began to be sustained by crossing more readily between different media and contexts. These crossings are the subject of Chapter 7.

\subsection{Challenging}

Once individuals in CoP A had developed confidence in contributing online, the facilitator moved to challenging them more directly. This was aimed at gaining alignment with the student-centred approach, as evidenced in this challenge to a teacher's use of clipart:

Could one of your students not [have] drawn a school bus? If you were busy could you have not turned the problem over to the older students to see how they would solve it? Could you have used a fancy title in Word art and put wheels on the bottom? (Facilitator, CoP A)

Continuing the crusade against clipart, the facilitator made a direct real-time online intervention into another teacher's classroom. The teacher concerned had just defended the use of clipart in a forum, promoting a teacher-driven approach:

One thing I do with clip art, being a junior teacher is use it for drawing! (shame) I can hear the tsk, tsk!? BUT if you ask a child to illustrate their poem about a camel and they don't know what a camel looks like, then what will the quality of the drawing be like?? What I do is get images from google and clipart and make up a page of about 10 pictures, enlarge it on the copier and then they can see how many legs it has, what sort of tail etc.(Teacher, CoP A)

In a variant of the side-conversation, the facilitator challenged this teacher-driven approach, in real time: 
I saw she was on i-chat and I asked her "Why do you do that, and what do you mean?" And she was saying, "Well, if I want them to draw a camel, they've never seen a camel, how do they know what a camel's like, unless I actually show them a picture of a camel, and that's how I do it.

This spontaneous interruption into practice was, on one hand, more discrete than a forum comment would have been - it avoided humiliation in front of peers; but on the other hand, it was a considerably more direct challenge; one that was difficult to avoid responding to. The teacher ("Sally") was successfully redirected in mid-practice:

....and so [Sally] was then talking to her kids and saying, "[the facilitator] wants to know about the camels. What are the things that camels have?" (Facilitator, CoP A)

\subsection{Piggybacking}

Piggybacking was a means of going deeper by building on the ideas of one or more others. It provided a more sophisticated means of aligning with the community than the agreeing and echoing that had occurred during Coming on Board.

We could go online and see what other teachers and principals - what their thoughts and comments were. We could hook into that, which for me was quite beneficial because...if you're not so clear on what's happening, you can see what others are talking about, and then it clarifies that you're on track, or gives you direction of where to go. (Teacher, CoP A)

When piggybacking it was necessary to interrogate the thinking of others before leveraging their ideas to help articulate one's own thinking. This fostered focusing while elevating thinking. One teacher portrayed piggybacking as a mental ladder-climbing exercise:

When you read a reading, you naturally form an opinion, [based] on...the knowledge you have... Once it goes online, people get the opportunity to feed back to you, and that's when the thinking begins, because all of a sudden somebody says, "Yes. I understand what you're saying, but have you thought about this?", or "I see that completely differently", and you're going, "Oh, okay..." and you can say "Yes, I agree", or "I disagree", or "I never thought of it like that, and if you think that, then I'm able to piggyback off what you've said", and then I can go to a new level: "Have you thought about this?"... and so between that, you're not in isolation. You haven't been able to have your thought and stick with it - It's actually promoted you to think, "Well, hang on". (Lead teacher, CoP A) 
As this deep, reflective thinking was underway, teachers had begun adapting their practice in the classrooms. This was, at least in part, a response to the cumulative impact of the online forums:

[By the third reading] people started to seriously think about the conversations.... and actually started to seriously think about doing other things, rather than trip up.

The facilitator of CoP A saw this as the starting point for the embedding of knowledge:

The moment that I start thinking about the modifications... and [then] start making some small change, or difference...then I'm starting to embed...

\subsubsection{Matching up theory and practice}

As teachers started going deeper, they considered the fit between their existing teaching methods and the student-centred paradigm - matching up theory and practice. This was prompted by discussions in online forums and face-to-face workshops:

You have the dialogue and the conversations, and...[then] you start actually thinking about what does this mean for me and my kids?

In forums, the emphasis shifted to identifying, celebrating and sharing things that teachers were already doing that supported the theory of the new way.

I am learning... to keep in mind the children who can make the technologies work when I can't and use the programmes to help each other which gives me time to help in other ways.

Teachers used reframing to chastise themselves for practices that did not align with it:

I confess the quick fix is sometimes the clipart...unfortunately I am probably cheating myself and the students with this route.

Sometimes, in the course of online discussions, they sometimes discovered that they were already doing things that fitted the new way, but had not previously recognised this. Matching up theory and practice helped them isolate which aspects of practice were relevant and which needed to be disembedded. In terms of the change journey, this practice helped teachers feel closer to their destination than they had at first imagined.

We discovered we were already doing bits of it, perhaps we didn't quite know why; we just thought it felt right. I think we learned a lot about the 
purpose of doing it that way.... The difference between a project and an inquiry; that it's got to have a meaningful outcome and something the children can take into the future with them, rather than just learning about something and that's the end of it...

Matching up theory and practice was an ongoing, iterative process that led to the gradual adaptation of teacher's practices and interpretive frameworks as gradual adjustments were made. It continued through the next two stages and is described in more depth in the next section.

\subsubsection{Identifying influencers}

During Setting Out, key individuals in each school - change agents and enthusiasts began a more sophisticated kind of piggybacking: They identified one or more online influencers, respected NZ and international educators, whose ideas they would increasingly follow. These influencers maintained an online presence in the form of a regularly updated edublog (educationally-focused weblog). Those who were based in $\mathrm{NZ}$ were core members of CoP E. I have called them connector-leaders, owing to the role they played in connecting communities and providing leadership. (I discuss this in depth in Chapter 8.)

Teachers followed influencers whose ideas struck a chord with them. Having the right kind of influencer was a way to ensure alignment with the new paradigm and gain authority for one's opinions. For change agents, there was particular value in aligning with those who kept in touch with educational research:

What I tend to do is look at people who I think are similar to me, or I find myself having similar views to them, and ...it gives a bit of weight to [my ideas], and often, what l've found also, is that - particularly with the inquiry learning and with the ICT... if you can justify your stand with a bit of research, then that gives just a bit more weight to what you're saying.

At a practical level, following influencers was a way of saving thinking time:

I'm a teacher, and I teach swimming and gymnastics and art and music and science and reading and maths and stuff, and their time is not fixed in that plane. They get time to exercise their minds on how things might be. So it's like saving me thinking time. They have more thought about it, so that I can learn from what they're doing.

Amongst the early influencers were the former facilitators of CoPs $B$ and $C^{16}$. These people published their thoughts about ICT in education, distilling relevant content that

\footnotetext{
${ }^{16}$ These people had moved on from these positions at the time of my visit, but their blogs were still being followed by teachers.
} 
was circulating amongst a larger network of edubloggers. The facilitators' blogs directed followers to resources, technological tips and the blogs of their own influencers; higher profile edubloggers who had a passion for the new way and/or ICT. There was a limited pool of international experts, and the community of local experts who blogged was even smaller, so change agents often followed the same sources. This redundancy in the system had a concentrating effect, increasing alignment amongst change agents. (This is further discussed in Chapter 8.)

\subsubsection{Summary}

During Setting Out, embedding of the new way began in earnest. Teachers piloted new models and methods and adapted their practice, exploring the implications of using ICT for student-centred learning. Knowledge of the new way became more personal and more practical. It was in a fluid state, as evidenced by the iterative matching up of theory and practice. Online CoPs played a key role, driving deeper engagement with ideas and fostering regular knowledge-sharing and problem-solving, Online interactions made visible like-minded individuals, leading to new buddy relationships. In CoP A, a critical enabler of embedding was the crossing over of the newly established online CoP culture, norms and themes into the workplace. This led to a new workplace culture of professional dialogue and peer support, promoting shared ownership of themes and the alignment of interpretive frameworks. Engagement in online forums was supplemented by the use and following of blogs by key members, leading to the identification of external online influencers. 


\subsubsection{Stage 4: Staying on Course}

The most competent teachers can come unstuck...you need to reaffirm and refocus...

During the fourth stage, Staying on Course, the key issue was maintaining both focus and momentum; moving forward while staying true to the spirit of the new way. Schools finished piloting and started adapting and contextualising their new learning models and methods. Individual teachers began developing fluency, adapting both their practice and methods that proved problematic in their pure form. Staying on Course required the continual planning of next steps and the seeking, applying and adapting of practical, technical and theoretical knowledge to facilitate understanding and delivery of the new way.

Teachers' desire for support and alignment was evident through peer-to-peer monitoring and benchmarking. As they contemplated their next steps, they relied on the online affirmation of buddies and the advice and opinions of influencers. There was an increase in the use of IM as teachers sought, received and applied practical and technical knowledge to aid delivery. Teachers engaged in self-monitoring, matching up theory and practice, reflecting on this online, and adjusting their practice.

Staying on Course was a transitional phase for the online CoPs. As the needs of individuals became more diverse and specific, a more complex CoP structure, with a tiered system of leading and following and online buddying relationships developed. There was a move away from a dependence on closed cluster-based forums, towards a more distributed and sustainable knowledge infrastructure based around blogs, with a community of mediating experts (CoP E) who directed relevant knowledge to change agents at each school.

This larger system-level infrastructure (described in Chapter 8) was better suited for supporting the increasingly specialised knowledge needs that had to be met in order for the new way to become embedded. By the end of this stage, knowledge of the new way was gaining considerable depth and richness in schools. The key embedding activities at this stage were planning next steps, monitoring and benchmarking, matching up theory and practice, buddying, leading and following, affirming, and contextualising (see table 11). 


\begin{tabular}{|c|c|c|}
\hline Activity & Description & Role of online community \\
\hline $\begin{array}{l}\text { Planning } \\
\text { next steps }\end{array}$ & $\begin{array}{l}\text { Planning the next steps in the } \\
\text { change journey }\end{array}$ & $\begin{array}{l}\text { Peers used IM to provide mutual advice on next steps, send links } \\
\text { to blogs/online content and suggest expert helpers. }\end{array}$ \\
\hline Monitoring & $\begin{array}{l}\text { Checking on progress against } \\
\text { goals and vision and fit with the } \\
\text { new paradigm }\end{array}$ & \multirow{2}{*}{$\begin{array}{l}\text { Teachers used IM to monitor and benchmark emerging practices } \\
\text { and promote alignment. }\end{array}$} \\
\hline $\begin{array}{l}\text { Bench- } \\
\text { marking }\end{array}$ & $\begin{array}{l}\text { Checking emerging ideas, } \\
\text { problems and practice against } \\
\text { those of others }\end{array}$ & \\
\hline Buddying & $\begin{array}{l}\text { Engaging in a sustained } \\
\text { professional relationship with a } \\
\text { buddy }\end{array}$ & $\begin{array}{l}\text { Peer-to-peer buddying was used for monitoring, benchmarking } \\
\text { and mentoring, affirming and planning next steps. This took } \\
\text { place in real time via IM and face to face; and via blogs and } \\
\text { email. It enhanced understanding of the new way in its } \\
\text { relationship to practice. }\end{array}$ \\
\hline $\begin{array}{l}\text { Matching } \\
\text { up theory } \\
\text { and practice }\end{array}$ & $\begin{array}{l}\text { Comparing theory with practice } \\
\text { and making adjustments }\end{array}$ & $\begin{array}{l}\text { Buddying via IM and/or blogging promoted the matching up of } \\
\text { theory and practice and leveraged complementary skills (e.g. } \\
\text { thinker/doer buddies) to foster this. } \\
\text { The online community culture encouraged individuals to identify } \\
\text { and share aspects of practice that fitted and/or did not fit, the } \\
\text { new way. }\end{array}$ \\
\hline $\begin{array}{l}\text { Leading and } \\
\text { following }\end{array}$ & $\begin{array}{l}\text { Following the ideas and advice } \\
\text { of chosen influencers and/or } \\
\text { providing leadership to others }\end{array}$ & $\begin{array}{l}\text { Some teachers followed the blogs of online influencers. Active } \\
\text { bloggers (connector-leaders) identified relevant content and fed } \\
\text { it to their followers, who fed it to so-called regular teachers. }\end{array}$ \\
\hline Affirming & $\begin{array}{l}\text { Reflecting in a way that affirms } \\
\text { the direction of change }\end{array}$ & $\begin{array}{l}\text { Teachers made affirming reflections on their changing practice } \\
\text { via IM, forums and blogs, providing "success landmarks". } \\
\text { Affirming built confidence, self-worth and motivation that } \\
\text { encouraged perseverance. Change agents made affirming } \\
\text { comments on each others blog, particularly if another change } \\
\text { agents had expressed doubts, frustration, or if shared values } \\
\text { were under threat. }\end{array}$ \\
\hline $\begin{array}{l}\text { Contextual- } \\
\text {-ising }\end{array}$ & $\begin{array}{l}\text { Changing a model or method to } \\
\text { make it better suit a particular } \\
\text { school context. This involved } \\
\text { adapting, simplifying and/or } \\
\text { integrating models. }\end{array}$ & $\begin{array}{l}\text { Teachers used IM to seek and provide ideas for "plugging gaps" } \\
\text { and adapting models. }\end{array}$ \\
\hline
\end{tabular}

Table 11: Stage 4 - Staying on Course 


\subsubsection{Planning next steps}

Schools and teachers now focused on planning next steps. This involved mapping out the route ahead, while staying true to the new way. Some participants emphasised that it was easy to get lost at this stage of the journey by falling prey to the lure of technology; losing touch with the student-centred approach.

People can get "hung up" on the way - they can get stuck on the tools instead of the learning...the technology is seductive, so you can get hooked on it. You keep hearing: "We've got a wiki...so we must be doing good stuff". (Facilitator)

In such cases it was necessary to refocus: "The most competent teachers can come unstuck. You have to stop - go Whoa! And "you need to reaffirm and refocus." (Teacher, CoP E). Refocusing involved the lead teacher or facilitator in intervening; encouraging teachers to take a clearer, more manageable focus, and bringing them back on board:

[You need to] take a smaller chunk of the snowball and start again...You need to say, "Maybe this chunk will help" and bring them on board [again] and say "We'll roll with this". And if that doesn't work, you try another chunk. (Teacher, CoP E)

Next steps typically involved breaking down, simplifying or revising a method that had not worked in the classroom.

\subsubsection{Monitoring and benchmarking}

A desire for collegial alignment and support was evident in the practice of peer-to-peer monitoring and benchmarking. As teachers contemplated their next steps, they relied on the affirmations of buddies and sought the advice and opinions of online influencers. Face-to-face discussions were supplemented by communications in invisible online contexts, using IM or email.

[I] What did you feel that they gained from that i-chat?

Support, I guess, and reinforcement, and also just someone else to chew the fat with really. And so that comes back to the support: you know, "Is my thinking on the right track? I'm just going to run this idea past you, do you think it's OK?" (Facilitator)

\subsubsection{Buddying}

Teachers further developed and leveraged the buddying relationships that had been established during the previous stage. This helped them stay motivated and identify their next steps. Two examples of buddying follow. 


\section{Example 1: Mentor buddying}

In the first example, lead teachers from different schools in CoP A, "Susan" and "Eric", reported on an extended dialogue concerning Susan's problems with WebQuests (an inquiry learning based method in which students gather information from websites).

What began as a forum dialogue was taken into a series of side-conversations traversing IM and face-to-face contexts. Because both teachers were change agents, it was important to avoid exposing Susan's doubts in the visible online forum.

I hated WebQuests with a vengeance. I had tried them in my room. It was a disaster.... And somebody i-chatted me. They'd seen what I put [on the forum], and they said, "Hey, I hear what you're saying, and this is what l've discovered"...."Hey, have you tried doing it this way? Have you tried doing it this way". And so, in actual fact, that went on for a long time... He'd obviously felt the same way that I did, but he'd pursued it as well. He said, "I agree, it's an absolute mess...you can't do WebQuests and teach your class because there's all the setting up, and you don't have a long enough time".

[I] So you had a common situation that he recognised?

Yes, definitely. But he'd made it work for him by putting these things in place... Because I'm a bit of a purist, I wanted to use it in its pure form. He said, "Take it apart. Download the stuff and print it off. I tell you what; we're not natives, we're immigrants. Print it off and give the kids a sheet. And then they can go online.

[I] So did you pursue that online contact?"

Yes, definitely

[i] But that was through i-chat?

Yes, definitely. Because the subjects we were supposed to be commenting on didn't involve WebQuests....

[I] So you had the formal agenda and the informal agenda?

Yeah.

[I] And did you use different media for those?

Yes...

('Susan')

In this case, buddying was an informal mentoring arrangement between equals; one of whom had more experience with a method. Eric provided encouragement and practical tips, helping Susan to de-emphasise technological issues and prioritise the student-driven inquiry process that underpinned learning. Their reported dialogue highlights the usefulness of side-conversations in staying on track and planning next steps. Nobody at Susan's school could have helped her, but once she achieved success, others followed her example. (It is interesting to note Eric's use of the digital immigrant motif to justify modifications that Susan sees as a dilution of pure practice.) 


\section{Example 2: Thinker-doer buddying}

The second example of how buddying facilitated staying on course comes from CoP E. Two innovative teachers - 'Diane' and 'Peter' - from different schools, developed a sustained online buddying relationship. Having met at a workshop, their original common ground was an interest in using interactive whiteboards. Both were committed to the new way but few of their school-based peers shared their degree of commitment and understanding. They interacted with each other daily via IM, blogs, Twitter and e-mail in a sustained, symbiotic dialogue. Diane was a self-confessed doer; an intuitive operator who occasionally found herself challenged to justify the rationale for her practice. At such times she was prone to a crisis of confidence:

It suddenly occurred to me that I needed to find out why this way of learning and teaching felt so right...It felt...urgent for me to redefine my own personal pedagogy to ensure that I wasn't just bombarding my students with the "bells, and whizzy stuff"...[l'm] feeling an increasing need to be able to justify why "that's what we do 'round here". (Diane's blog)

Peter, on the other hand was a thinker. He was prone to deep introspection about the educational theory behind the new way, but sometimes faced problems with motivation.

All I have done today is my presentation... It didn't go...so well...Sometimes when I'm in school and I'm going on and on about the importance of technology and the changing model of classroom practice, people either glaze over or tell me l'm talking rubbish. Sometimes you just need a real person to sit beside and encourage each other on this journey. I also think it'll be good for...twittering... (Peter, blog post)

Peter and Diane's online relationship had mutual benefits. Peter helped Diane think more critically about her practice, interrogating the rationale behind it. This had a grounding effect, focusing her on making adjustments to improve engagement and learning:

[Peter's] the thinker, and I'm the doer, and I think that that's probably why... our relationship works so well. He really examines why we're doing things... He's made me stop and think about what are the reasons behind doing this, why is this a good thing to do, what's the motivation behind doing it? Is it really good teaching practice to be doing this? Is this really going to help the students learn? ... He grounds me, because I get quite carried away... I plan all these wild and wonderful and wacky things, and sometimes I just go overboard. I'm discovering...that that's really put a lot of my students off. Like they're not as enthusiastic or as excited about ICT as I am...And so he's been really good helping me slow down.... it's like yin and yang." (Diane) 
Diane, in return, helped Peter stay on course by affirming his successes.

Well done you for putting into practise what we heard from [the speaker]...I am seriously considering it now - especially if the independence improves in our class as much as it has in yours! (Diane; comment on Peter's blog)

While Diane saw Peter as grounding her, Peter saw Diane as dragging him up: She provided him with time-saving practical knowledge and guided his next steps in using technology.

She's had a huge impact on dragging me up, in terms of my professional practice.... It's great...I don't spend until three in the morning trailing through the Internet, because [Diane] does it for me. She goes and tries stuff out. She'll have a conversation... with people around the world, and they share their ideas with her, but... She comes back and tells me things like the voice-thread, for example. She helped me with podcasting in my classroom. She directs me towards online applications... She's very good at "Oh, this will be good for...something in particular." (Peter)

This buddying arrangement provided long-term benefits, contributing to each teacher's understanding of how to effectively enact the new way. By developing as buddies, Diane and Peter expanded their common ground while retaining a clear focus, continually aligning their emerging practice with the new way. They also gained confidence from addressing each other's weaknesses. In a comment on Peter's blog, Diane noted:

It is always a pleasure to bounce ideas around with YOU! You keep me grounded (so I don't go off in a huge rip,roar and bust tangent!!) I guess we have similar goals, similar passions and whilst we're [teaching] at different levels we have the ability to see where we are going or where we have been and that is what makes our collaboration work!

The above two examples of online buddying did not just benefit the individuals involved. They also contributed to school-level KT and embedding by improving the knowledge and capabilities of those who were change agents: Susan gained useful practically oriented knowledge and the confidence to persist and enlist help if she encountered barriers. Eric gained experience in mentoring and articulating the value of his adaptation. Diane gained a stronger student focus and an ability to justify the methods she was promoting; while Peter gained know-how, confidence and motivation to persist as a change agent. 
Further benefit to schools came from the value and persuasive power that accrued in buddies' blogs over time. Both Diane and Peter had blogs that were followed by others in their schools - they were connector-leaders who positioned themselves as fallible learners. This can be seen as lowering the barriers to the engagement of their peers.

\subsubsection{Matching up theory and practice}

During Staying on Course, teachers undertook self-monitoring through the matching up of theory and practice that had begun in the previous stage. They reflected on the fit of their teaching with the principles of the new paradigm then made practical adjustments.

For me it was looking at the theory of teaching and the practice.... the theory backed up what you were doing practically, and if you were doing something practically, you could often match it up with the theory...

This process has also been exhibited in Diane's interactions with Eric. Reflecting on the theory underpinning the new way helped teachers to structure thinking and firm up their beliefs. Sometimes this exercise affirmed what they were doing intuitively, in their classrooms:

It was a case of (realising), "Oh, so this is how I do things. Oh this is what it's called, and this is why I do it like that."... It was sort of affirming and putting a title on the way I did things.

At other times it exposed a conflict between the theory of the new way and the reality of practice, motivating teachers to seek resolution:

We saw that what we were doing wasn't really what we wanted to be doing, so [we thought] "How do we go about changing that?"

The ongoing professional discussions, spanning online forums, IM and the workplace, played a key part in facilitating this iterative matching up process:

That link between theory and practice, it was never there before, but through those discussions, I was able to make that link...

Blogs provided an ideal context for reflecting on, and affirming, practice and beliefs. Unlike forums, blogs encouraged the building of value over time around eclectic interests. The practices of embedding links and tagging content (social bookmarking) aligned these personalised repositories with the blogs of global influencers. Teachers who were bloggers often reflected on events in light of their fit with the new way. They 
followed others who did likewise, helping to reinforce and extend their developing knowledge.

He made some great points about the 'new classroom' that fitted into my ideas and extended my thinking about pedagogy. (blog)

\subsubsection{Leading and following}

A complex system of leading and following developed during this stage as existing roles evolved and new, unofficial roles emerged. This was driven by a growth and diversification of knowledge needs and resulted in a more distributed online knowledge infrastructure, as key members of CoPs A, B and C joined CoP E.

Lead teachers become in-school experts and mentors as the emphasis of their role shifted from persuading to supporting teachers in adapting. As school needs became more specific and diverse, lead teachers needed access to external solutions and ideas. Their dependence on facilitators was complemented by a growing reliance on the edublogging community ( $\mathrm{COP} E$ ) and network to which the facilitators belonged. Some lead teachers became follower-feeders, peripheral members of CoP E. They regularly followed selected bloggers, but avoided leaving comments on their blogs, using side-conversations to communicate with them. (Figure 27 shows an excerpt from a follower's email.) When they gained useful information they fed it to regular teachers, often in face-to-face contexts.

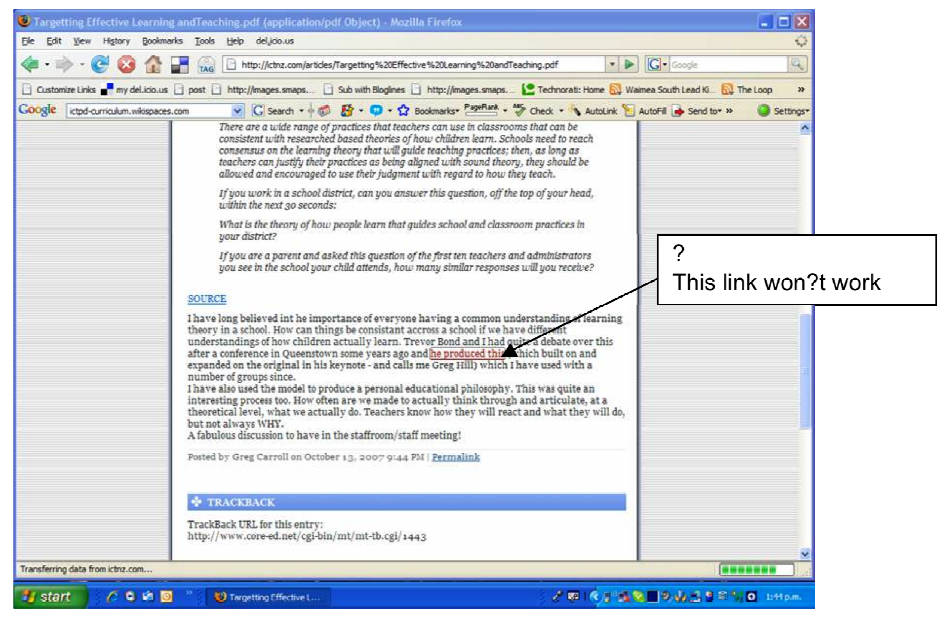

Figure 27: Invisible e-mail to a connector-leader from a follower (CoP E)

A smaller number of lead teachers (1-2 per cluster) became active members of CoP E. They also followed influencers, but communicated directly on blogs (and also via IM and email) creating an online presence. Over time they began to publish their own 
blogs, using RSS feeds and tagging to interact with higher profile bloggers. This was a little like being a fan:

They're out of my league! They're big names. On their cluster map on their blogs, all you see is red, swathes of red painted everywhere (see figure 28).
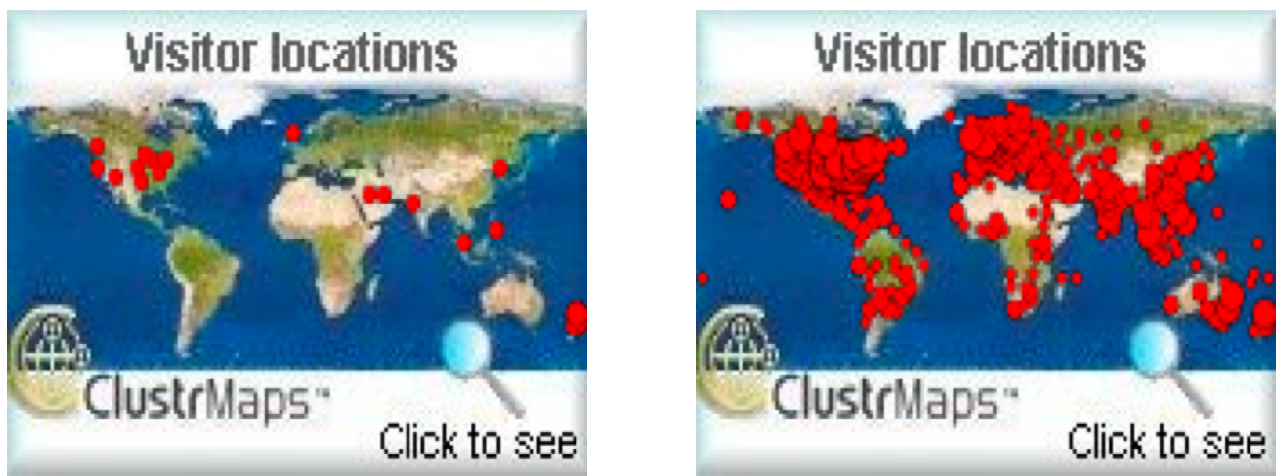

Figure 28: Cluster maps (blog widget) showing readership of a CoP E member's blog (left) compared with the blog of a global influencer (right).

Publishing one's own edublog ${ }^{17}$ was described as "moving into a bigger pond" or "joining the conversation". Those who did this became connector-leaders, core members of CoP E.

The differentiation of roles described above resulted in a stratified system of leaderfollower interactions. This is shown in figure 29 along with the communication contexts within which these interactions occurred. Regular teachers followed follower-feeders, who followed connector-leaders, who in turn followed global connector-leaders). As this system evolved, connector-leaders and follower-feeders became important knowledge brokers who were instrumental in facilitating embedding at a macro, or system, level. (I explain this in depth in Chapter 8).

\footnotetext{
${ }^{17}$ During the period of this study there was something of an explosion of edublogging internationally. This was reflected in NZ, with blogging being promoted at the annual ICT PD conference. These factors no doubt contributed to the growth in COP E.
} 


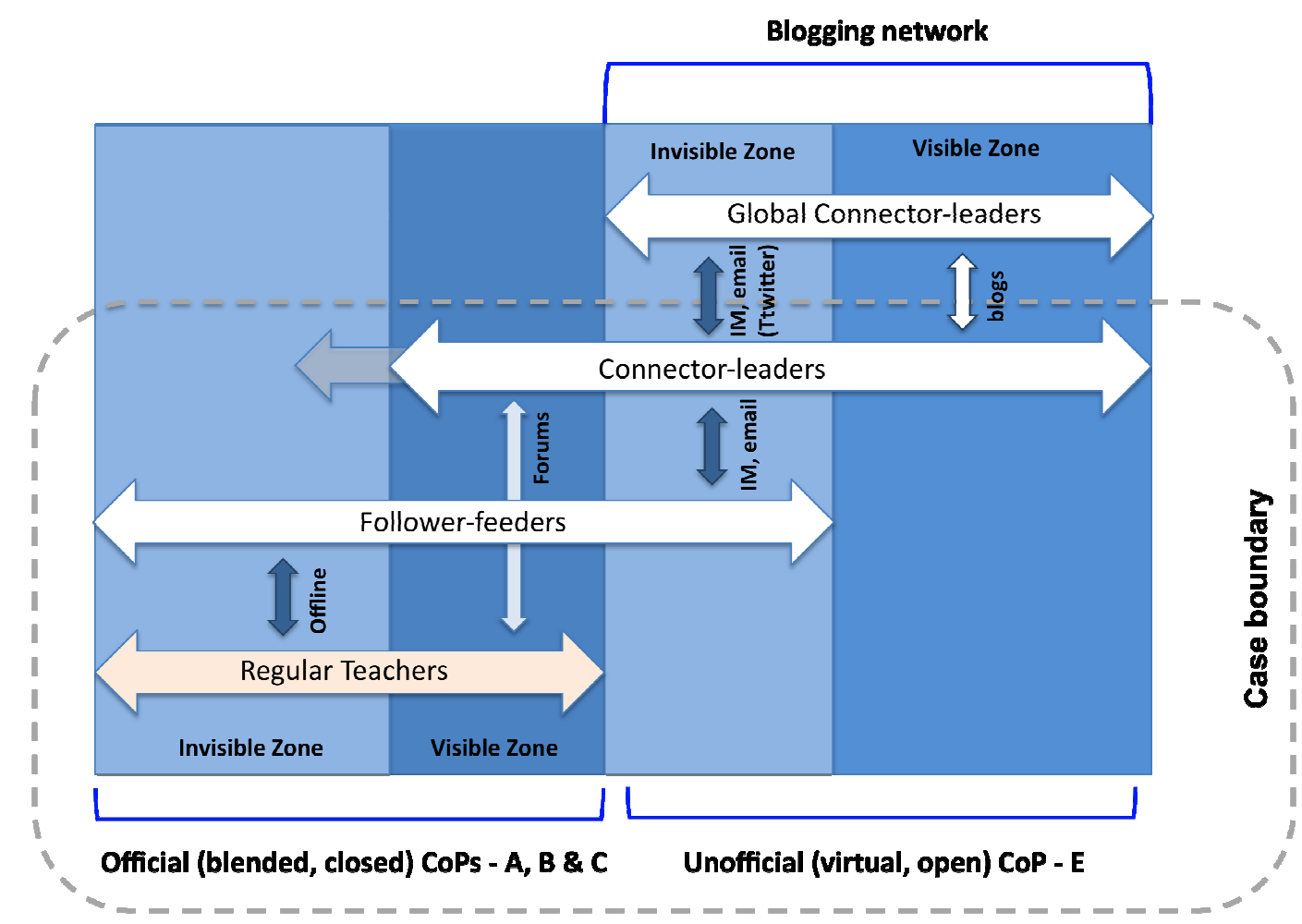

Figure 29: Tiered leader-follower relationships showing visible and invisible communciation zones

\subsubsection{Affirming}

During Staying on Course, change agents made affirmations about the progress they had made. Affirming was a form of persuading that legitimised efforts made to date and built a sense of pride. Online affirmations, made in forums and blogs, and via IM and Twitter, created landmarks of success, providing motivation to continue the journey and persevere:

All but 4 in my class are now reading 8+yrs, ALL are within a 6 month range of their age yippee! Must be the technology in my room... (Twitter)

Affirmations sometimes related to the value of the online community in facilitating collaborative development.

I have also been investigating Flickr $\mathrm{CC}$ of late - we are all on a learning journey - I agree that together we will learn more. Keep up your awesome work! (Collegial affirmation, blog)

Change agents also provided their peers with affirming comments, particularly if a colleague expressed doubts or was challenged. The need for collegial affirmation is clear in the following teacher's expression of isolation and frustration: 
It is really difficult to be a staff member on your own in a school where people don't 'get it'... I feel like an island, surrounded by water and sometimes drowning. (Blog)

Community members responded rapidly to such incidents:

I know how you feel. Far too often I find the system is too slow to accept change. (Blog)

\subsubsection{The changing knowledge infrastructure}

Staying on Course was a transitional phase during which the centralised, official online community infrastructure, based around forums, began to be supplanted by a more complex, sustainable, distributed, non-official online community infrastructure, based largely around blogs. Reliance on IM continued and was supplemented by the use of Twitter.

During the early stages, when schools' needs had been more uniform, online forums had spearheaded change by building common ground, facilitating communal reframing, establishing new norms, and persuading teachers of the value of the new way. However, forums were a less suitable means of supporting schools and individuals during the Staying on Course stage. Knowledge needs were becoming increasingly diverse and teachers needed more personalised support for adapting. The distributed structure of the blogging community, and its connection to a network with multiple pockets of expertise, was better suited to supporting these needs. As schools recognised this, there was a shift from full dependence on the facilitator to reliance on a more distributed knowledge base; CoP E's connector-leaders and their connections. Staying on Course required the voyagers to expand their focus beyond the vessel on which they were travelling and to start communicating with a larger fleet of travellers, and with those on the shores ahead.

\subsubsection{Contextualising}

Staying On Course also involved schools in contextualising: adapting models based on the new way to better fit their needs. Teachers were charged with bringing back what they had learned, helping to establish preferred school models. In some cases, this involved simplifying or streamlining models:

There was no doubt that it worked for us. It was more the fact that maybe we found it a little bit awkward....we wanted [it to be] a little bit...more simplistic. ...We thought it wasn't as good as what possibly we could have... 
In other cases contextualising involved integrating ideas to plug gaps that had been identified.

We'd been using [one] model for a while, and then I tried the Big 6 or Super 3 for Juniors model, which is quite popular. And they were OK, but there were gaps that we felt we needed to plug.

The online community provided an ideal way to canvas colleagues and resolve the problem:

Just by that on-line community discussing what other people were doing, we were able to find things that would probably plug our gaps...

Lead teachers also began aggregating the models and methods that had been tried and found successful, storing them in online community repositories. The aim was to provide examples of what worked for others to emulate.

\subsubsection{Summary}

During Staying on Course there was a stronger need for external guidance and support than at any other stage. This was reflected in an increased reliance on the online dimension of community and the move towards a distributed knowledge and leadership infrastructure. The emphasis was on individuals maintaining focus and staying on track - aligning with the new way and each other - while gathering the knowledge they needed to adapt their emerging practice and secure classroom gains. The result was that knowledge of the new way developed depth, diversity and richness. 


\subsubsection{Stage 5: Anchoring}

And then we formulated our own school model. The staff brought

back all that learning from way, way out, right back into what was best for us.

In the Anchoring stage, schools arrived at the end of their change journey and began anchoring the new knowledge in place. They focused their attention and energy strongly inwards, working on customising, formalising, aggregating and routinising their new teaching approaches. Anchoring was about the conscious, structured embedding and rolling out of newly articulated knowledge at school level. It can be seen as analogous to the start of the (re)freezing stage of Lewin's (1952) model of organisational change.

As schools anchored, they increasingly differentiated themselves from one another. Each school firmed up its own approach to the new way, documenting customised teaching models and methods and rolling them out. As a result of this differentiation and internal focus, their reliance on the official online community diminished. The shared online space became primarily a repository for models, effective practices and professional readings. However, the continued impact of the online CoP was apparent. Once-challenging language that had been cultivated in online forums was now normalised and integrated into day-to-day workplace talk, while the online forum records were still seen as a valuable resource. ("It was quite useful going back... because it was always there, and you could go back and look at something else..."). This is reminiscent of Wenger's (1998a) Memorable stage of CoP development, but did not signal the end of the informal online CoP.

Individuals continued to rely on the unofficial online community, and their online buddies, as they refined their teaching approach, developing stronger personal understandings of the new way. (This was strongly evident in CoP E, where change agents reflected on, affirmed and defended their changing practice on blogs.) They also continued to provide assistance to one another via IM and Twitter. (These activities are summarised here and outlined in more depth in Chapter 7.)

The key embedding activities during Anchoring were customising, formalising, aggregating, routinising, personalising and improvising (developing fluency). In 
addition, the following activities from the previous stage continued: Matching up theory and practice, Buddying, Leading and following and Affirming (see table 12).

\begin{tabular}{|c|c|c|}
\hline Activity & Description & Role of online community \\
\hline Customising & $\begin{array}{l}\text { Developing school-specific models and } \\
\text { approaches by adapting and/or } \\
\text { integrating existing ones }\end{array}$ & \multirow[t]{3}{*}{$\begin{array}{l}\text { Schools used the online community as a repository } \\
\text { for aggregating and formalising customised models. }\end{array}$} \\
\hline Formalising & $\begin{array}{l}\text { Documenting and approving models } \\
\text { for school-wide use }\end{array}$ & \\
\hline Aggregating & $\begin{array}{l}\text { Drawing together information and } \\
\text { models }\end{array}$ & \\
\hline Routinising & $\begin{array}{l}\text { Making routine use of the new } \\
\text { knowledge (embodied in models, } \\
\text { methods and language) }\end{array}$ & $\begin{array}{l}\text { Routinising began in syndicates, building on the } \\
\text { positive dynamic that had been established in the } \\
\text { online CoP, before being rolled out. }\end{array}$ \\
\hline Personalising & $\begin{array}{l}\text { Developing personal understanding } \\
\text { and use of a school-based method or } \\
\text { approach }\end{array}$ & $\begin{array}{l}\text { The history of online discussion placed teachers on a } \\
\text { "level playing field", allowing them to personalise } \\
\text { approaches while maintaining alignment with peers. } \\
\text { Forum records were used retrospectively to build } \\
\text { and reinforce understanding. Blogs provided } \\
\text { visibility to emerging practice of peers, providing } \\
\text { models and inspiration. }\end{array}$ \\
\hline Improvising & $\begin{array}{l}\text { Applying knowledge to adapt and } \\
\text { redirect teaching on-the-fly. This } \\
\text { required rapid matching of situations } \\
\text { to methods/theory. }\end{array}$ & $\begin{array}{l}\text { Teachers in CoP E reflected on improvising in } \\
\text { persuasive postings on their blogs, connecting } \\
\text { improvisations with theory of the new way and } \\
\text { noting positive outcomes. This promoted } \\
\text { understanding, affirmed value and created } \\
\text { motivation. It also attracted the online affirmations } \\
\text { of others. }\end{array}$ \\
\hline $\begin{array}{l}\text { Matching up } \\
\text { theory and } \\
\text { practice }\end{array}$ & See table 11 & \\
\hline Buddying & See table 11 & \\
\hline $\begin{array}{l}\text { Leading and } \\
\text { following }\end{array}$ & See table 11 & \\
\hline Affirming & See table 11 & \\
\hline
\end{tabular}

Table 12: Stage 5 - Anchoring 


\subsubsection{Customising}

Schools used their understanding of the new way and their unique needs to adapt the models they had trialled, developing customised approaches and toolkits (sets of approved teaching and learning tools) for school-wide use. This differentiated them more strongly from each other.

The other schools [in the cluster] haven't become clones of us...they've actually become their own school, and made their own decisions, and they've taken it where they wanted it to go...embedding of inquiry, but with a willingness to take it where your school and you believe it should go, not to go where [we've] gone.

There were two benefits of customising a learning model (versus using a generic one): Firstly, customisation ensured workability. The model was tailored to each school context, taking into account the results of piloting by its own teachers and the wider community's input where problems and gaps had been identified. Considerable knowledge was therefore embedded in it. Secondly, the collaborative process of customising promoted collective and individual ownership:

When you look at the models that are available, they do have lots of similarities. There's the immersion stage and the brainstorming [stage] and all the other stages. But we didn't want our model to be linear, so ... we made ours look like a tree with branches, so that you can actually rotate and go backwards and forwards... (Teacher, CoP A)

\subsubsection{Formalising}

Formalising models and approaches involved documenting them and mandating them for whole-of-school use. This was the visible outcome, and the endpoint, of the official online community: Once issues that arose during piloting had been resolved, there was no longer a need for community revision of the models. Formalising also impacted on planning:

At the beginning...we were doing so many science, so many social studies, so many health [units], in a certain time-frame and...everyone was going to sit down and study ants or whatever, and it was all level appropriate. Whereas now, I believe, we look at what we're delivering in relation to our school programme, in relation to our students' needs, and then we break that down into what individual children need, what their interests are; we're far more focussed about the children taking responsibility for their own learning. (Principal, CoP A).

\subsubsection{Aggregating}

Schools aggregated the models and methods that had been piloted and adapted, storing them in online repositories (the internet and/or online community space) with attributions to the teachers who were most familiar with them. This material included 
diagrams and practical tips. The formal site also contained links to materials that had been aggregated on blogs of lead teachers. For example, a teacher in CoP B had created an extended blog post capturing models that had been presented at a full-day professional development workshop. This was intended to provide an after-event focal point.

Hopefully this blog post will become an interesting focal point for the ongoing discussion of the thinking tools that we learnt today.

Such blog-based repositories provided complementary benefits to the more formal records: The featured models were interwoven with valuable reflective commentary and hyper-links.

\subsubsection{Routinising}

The formalised approaches started to be integrated into conscious routines. This typically began in the syndicates to which lead teachers belonged. The intention was to demonstrate success in that context, then roll it out more widely to get school-wide consistency.

We're doing inquiry units here at our syndicate, and that's just awesome, because they're learning so much.... it's more child-centred, less teacher-directed, and learning together. (Lead teacher, CoP A)

We're... trying to push these ideas out there, so we're getting consistency across the school. (Lead teacher, CoP B)

Routinising was a largely conscious activity, but this changed in the next stage.

\subsubsection{Personalising and improvising}

Within each school's chosen approach, teachers developed personalised understandings and methods. For example, if using inquiry learning, they adapted it in a way they felt was appropriate for their students, incorporating the use of blogs, podcasts, IM, websites, digital cameras, or drawing software. Teachers in a school were able to personalise their approach while maintaining alignment with their peers because, as one interviewee put it, they were on a level playing field, with a set of shared repertoires and meanings from which to draw:

We've all got the same background, and we've taken our own bent we've got that sort of scaffolding... and we all know what we're talking about. So when we talk about how we plan, or I come with some new idea... I know the basis of how it should work, because l've used it before and I know what's good about using it... It's made us think about what we can use at our school, and how we can use it within the age- 
group that we're teaching...we're all sort of on a level playing field, because we've all had the same background of shared discussion. (Lead teacher, CoP A)

By encouraging personalising, schools recognised the professionalism of teachers:

[We're making] a folder that gives guidance as to inquiry and graphic organisers; what's used in the school, and where it's used, so that we've got a consistency, so that we all understand what's happening, basically, in each room. But at the same time each teacher still knows that it's not a constraint. Just because they've got these graphic organisers in the system, it doesn't mean they can't pull out another one and use it. They've got a license to use their character as they like. (Principal, CoP B)

This was a very different situation from the school-centric approach outlined by a teacher in CoP E:

Everybody does the same reading, everybody does the same writing, everybody has the same model for maths, and all of those things, and we do it all in the same way, and you can see children's progress because we can; because we are delivering it in the same way....

Personalising included planning the application of a model, testing the approach, reflecting on its success, and iteratively improving it. This sometimes involved combining different techniques. One teacher spoke of using role-play to kick-start an inquiry learning process in which students used the web.

I thought, well, if I develop this model... it's a whole bunch of communication things going on...they're going to have to work in small groups, there's a whole lot of...higher-order thinking incorporated into it... (Teacher, CoP E)

As teachers developed fluency, they complemented this with improvising. This involved letting go of preconceived plans and habituated responses (disembedding) in order to enact the new way more intuitively, in response to students' learning needs, through on-the-fly decision-making. Examples of improvising, recounted on blogs, are provided in Chapter 7. In one case, a teacher allows a reading class to morph into an investigation when students express curiosity about the difference between crocodiles and alligators. In another, the teacher reframes learning to spell as a game with a level boss who must be defeated.

The fact that teachers were able to apply their new knowledge by improvising indicates that some embedding had occurred. Relevant knowledge was readily accessible, and 
teachers were able to match it up with real-time situations to create instant solutions. These episodes were examples of Schön's (1983) reflection in action. They also illustrate how embedding and disembedding (Szulanski, 2000), or learning and unlearning (Malhotra, 2002) occur hand in hand. While online CoPs did not play a role in these classroom events, teachers' later reflection on these episodes - notably in blogs - was significant.

These recorded episodes made the classroom practice of change agents (role models) visible to colleagues, providing ideas and inspiration. They were persuasive stories, typically including: (a) a story about how the teacher's diversion from their planned or habituated script to follow a student-centred approach, (b) a (proud) statement about the impact of this on students, (c) an affirmation of the teaching/learning act; interpreting it in light of the new way - matching of theory and practice. Combining these elements was a powerful embedding act on the authors' part, helping cement understanding of, and belief in, the new way. The blog posts exemplify reflection on action (Schön, ibid) and what I term theory-practice crossings (see Chapter 7).

A number of teachers in CoP B ran classroom blogs. These were student-authored records of classroom learning activities and not seen as part of the online CoP. However, they provided a window on their personal practice and became a source of applied knowledge and inspiration for their colleagues:

[I] Do you all keep an eye on each other's classroom blogs?

Yes...You know which ones to look at: the [teachers] that are doing things... I think, "Oh, that's really good" or "I've done that" or "I've done something, "We could try that"... It just helps you to try different things, and gives you different ideas.

\subsubsection{Affirming}

Self-affirmations were made through the online telling of success stories, as discussed above. These affirmations were often seconded. School-based followers provided positive feedback by email, IM, or in face-to-face settings. Core members of CoP E made affirming comments on the blog, sometimes offering suggestions for refining the practice:

It was a good idea to spell out the practicalities of what you do in the classroom. Sometimes people just need to see the small steps involved... I'm like you. I definitely have a play-time before I introduce anything to the kids. And I think it is equally as important for the kids to have a play-time when we introduce something new to them... 
This kind of feedback reinforced the value of the new practice, identified others pursuing a similar approach (aligning), and motivated the authors to pursue the practice and continue their blogging; an entirely voluntary leadership role. One teacher noted, "it's a huge buzz when someone engages with what I am thinking about and blogging about."

\subsubsection{Summary}

During Anchoring, knowledge of the new way became more unified, customised and explicit as it was captured; and more routinised as it was embedded in schools' planning and teaching approaches. The knowledge being anchored was the outcome of the online and offline community activities that had occurred during previous stages. The official online CoP now became a repository of models reflecting each school's approach. Online forum records were valued historical resources that provided reminders and continued to promote understanding.

As they developed customised school-wide approaches, becoming more differentiated, schools developed a strong sense of owning the new knowledge. This came with a sense of depth, or profundity, of shared understanding, as noted in one school's milestone report:

Having the opportunity to learn about questioning and inquiry learning is a very valuable use of lead teacher time. It has given us freedom to experiment with a new and challenging teaching tool, and has encouraged us to think about what education might be like in the future. Because of this, we have begun the process of changing and modifying our programmes to best suit the students, while ongoing staff discussion has developed a profundity of knowledge on a school wide basis. (School Milestone Report, CoP B)

A school-wide sense of professionalism was becoming embedded:

Now we're much more child-centred, we're much more professional in the way that we look at each other, we're much more rigorous in our debates that we have, we're more open to the doing things, new ideas we're much more open to new ideas. (Lead teacher, CoP A)

At the individual level, knowledge of the new way as becoming more personalised. In CoP B, classroom blogs gave online visibility to the emerging personal practice of peers, promoting mutual inspiration and alignment. 


\subsubsection{Stage 6: Settling}

That is the way now - Inquiry Learning is the way!

In the final stage, Settling, schools focused on cementing the new knowledge in place and becoming more comfortable with $\mathrm{it}^{18}$. The aim was institutionalising their version of the new way - aligning it across the school and embedding it both in routines and in the school's identity. This stage can be seen as analogous with Lewin's freezing.

As routinising continued, the 'new' teaching and planning approaches became more widely used across schools and more deeply integrated into practice, as teachers modified them for use with different subjects and/or age groups. Some schools began creating strategic documents to capture their new approach: These documents represented the maturing and flowering of ideas that had been seeded during early online discussions, and which had been nurtured and enriched during the journey. Along with the interview data, this material evidenced the fact that the new knowledge was seen as paradigmatic: a new approach to teaching and learning. It was now so strongly owned and well understood by schools that they were ready to explain it to stakeholders.

During this stage, knowledge of the new way became embedded in conscious and unconscious routines, interpretive frameworks and even identities. The new knowledge was also inextricably linked with a belief in its rightness.

Settling was also "catch-up time". Stopping change and guarding against new inputs provided time for teachers to work at mastering the new practices, and for latecomers to be brought into line. Schools consolidated gains by selective recruitment of staff.

For change agents, Settling and its cessation of change represented a paradox: On one hand, settling time was needed in order to embed knowledge more deeply and widely, but on the other hand, settling for too long was seen as risking stagnation.

\footnotetext{
${ }^{18}$ The four schools in CoP A were in the Settling stage and two in COP B were entering it. In addition, a key member of CoP D provided valuable data about longer-term settling.
} 
Key embedding activities during Settling were institutionalising, guarding, refining, refreshing, routinising, internalising, consolidating, and reviewing the journey (see table 13).

Although informal online collaboration continued among change agents and their followers, it played little role in supporting Settling.

\begin{tabular}{|c|c|c|}
\hline Activity & Description & Role of online community \\
\hline Institutionalising & $\begin{array}{l}\text { Making the knowledge part of } \\
\text { the organisation and its identity } \\
\text { through formalising and } \\
\text { normalising }\end{array}$ & $\begin{array}{l}\text { The online CoP became an institutional repository. In one } \\
\text { school, the online space was used to collaboratively } \\
\text { develop strategic documents. These represented the } \\
\text { outcome of ideas cultivated in the online CoP. }\end{array}$ \\
\hline Guarding & $\begin{array}{l}\text { Protecting teachers from new } \\
\text { demands and inputs to allow } \\
\text { catch-up time }\end{array}$ & N/A \\
\hline Consolidating & $\begin{array}{l}\text { Shoring up gains made by } \\
\text { refreshing knowledge, setting } \\
\text { expectations and selective } \\
\text { recruitment }\end{array}$ & \multirow[t]{3}{*}{$\begin{array}{l}\text { Teachers reviewed forum records to refresh their } \\
\text { memories. Re-reading discussions in the light of } \\
\text { experience resulted in new connections being made. }\end{array}$} \\
\hline Refining & $\begin{array}{l}\text { Making minor adaptations to } \\
\text { models/practices, for example } \\
\text { applying them in new subjects } \\
\text { or for new groups of students }\end{array}$ & \\
\hline Routinising & See table 12 & \\
\hline Internalising & $\begin{array}{l}\text { Integrating knowledge into } \\
\text { interpretive frameworks, } \\
\text { routines and practices so it } \\
\text { becomes a tacit (unconscious) } \\
\text { part of practice and identity }\end{array}$ & $\begin{array}{l}\text { Internalising was the outcome of a complex interplay of } \\
\text { factors, including participation in online communities. }\end{array}$ \\
\hline $\begin{array}{l}\text { Reviewing the } \\
\text { Journey }\end{array}$ & $\begin{array}{l}\text { Reviewing and celebrating the } \\
\text { change journey and gains made }\end{array}$ & $\begin{array}{l}\text { Schools recognised that online CoPs had played a part in } \\
\text { embedding their new knowledge, but underestimated } \\
\text { their impact. }\end{array}$ \\
\hline
\end{tabular}

Table 13: Stage 6 - Settling

\subsubsection{Institutionalising}

Schools began institutionalising - integrating, formalising and normalising the knowledge they had gained at higher, structural and strategic, levels. One principal described changing a syndicated staffing structure to one based around areas of natural strengths and interests, to better support students. Two schools had begun 
developing new visions, mission statements and even curricula. (Developing a curriculum was described as "taking the bull by the horns" by one principal: schoolspecific curricula were not yet mandatory.) Formalising the new knowledge at a strategic level in some way was nonetheless seen as a critical step by most schools. Leaders saw it as necessary to align the public face of the school - what parents read about and understood - with what was actually happening:

The reality is we no longer teach social studies, science, health, technology; they're all in one. And we actually need to write it, and say this is what we do, and more importantly, this is why we do it... If I presented you [with] my document now, you would assume that we taught [everything] in the old way; you know, everybody just sat in the classroom, so we need to [formalise] it .... We've spent three years discussing what beliefs are, and what teaching really is, and how ICT can support it. Now we actually need to firm up some ideas. So "At [this school], what is teaching and learning?" And "If you like it, Mum and Dad, this is the school for you; if you don't, you need to go and find somewhere else." (Principal, CoP A)

In one school, the new way was strongly embedded in new strategic documents. The impact of the earlier online community activities on this 'downstream' material was clear when I interviewed a lead teacher who worked on the documents: He did not hesitate before attributing the bulk of content to the online CoP, associated readings, forums and blogs:

We went right back to looking at our mission statement, rewriting it.

[I] So that happened while you were doing the ICT [PD programme]? Has some of that thinking fed into it?

Most of it... We wouldn't have gone to the depth that we did had we not had those discussions and that professional development in those online communities through the cluster.

[I] So in the mission statement, where would you point your finger?

[The teacher points...]

Just even going to Effective Learners, Effective Citizens, the core sort of stuff, our curriculum beliefs - What we Believe, all these are the result of discussions we had as a staff, but also from the things that we were either reading about online [or the facilitator] was introducing us to...Defining our Purpose of Inquiry, down to Our Vision, looking at Planning, Teaching and Assessment. All this here. Our reporting, our portfolio work was the result of stuff that we'd found as a result of going to that blog...All this stuff here is the result.

Although institutionalising suggests rigidity, this was not the case, as was made clear by a principal in CoP B: 
At [this] school it used to be... at Thursday afternoon at two-thirty, the children would be working on this worksheet. It was all very programmed in and everything was in tidy boxes. Now inquiry [learning] has taken teachers right out of their comfort zone, which is where learning happens, and now we don't know what we're going to be doing on Tuesday at two o'clock, because inquiry will take us in several different ways. And so I think teachers have relaxed, and are willing to let the children take the topic where it wants to go. That's a huge shift in practice.

\subsubsection{Catch-up time: Guarding and consolidating}

In order to facilitate deeper embedding, school began guarding against new inputs in order to consolidate gains. A teacher described this as catch-up time:

I believe that now is...catch-up time. It's..."Hey I achieved this. I know about this, and now I need to pull it into my classroom practice, and so I'm going to use it this way, and this way, and this way"...

Catch-up time was used for mastering new the approaches and bringing latecomers up to speed. During this period, teachers returned to online forum records to refresh their memories and understandings. Re-reading online discussions in the light of their experience could result in new connections being made.

I still have to feed myself with going back and reading again... refreshing the whole thing - [reminding myself] what it's about, and refining it...

One principal spoke of backing off:

The goal this year was just to sit back and acknowledge the fact that we'd done a lot, and embed just what we'd done, and back off for the teachers.

Using the journey metaphor, one lead teacher emphasised the need to keep an even kee/ while Settling occurred:

We're sort of on an even keel. We don't want to lose our staff. We want to keep them on board, so just little steps.

Where reluctants had resigned, principals sought out teachers with experience in the student-centred use of ICT who would be a better fit. They also began to communicate clear expectations:

It's just carrying on and making sure that it's at the forefront...part of their discussions, and it's part of what's expected. 


\subsubsection{Refining}

Teachers who had mastered the new approaches started refining them for use with different groups of students and/or subject areas:

Consistency within a school ...may make sense but...it's really important ...to make adaptations. These adaptations may just be for some children who require more support in certain areas... (Facilitator, CoP A)

Principals undertook regular benchmarking with each other, comparing notes and refining their approaches:

Just little pieces allow you to go back and adapt...sometimes it's just listening and hearing what they're doing in their school and thinking, "I'm doing that", so you feel good, or you think, "Oh, I hadn't thought of that. I might try that in my school". (Principal, CoP A)

\subsubsection{Routinising and internalising}

Schools continued routinising the new approaches; for example by using inquiry learning in more structured ways:

(It's) now been adopted school-wide: All classes are using it. It's on the wall in each classroom, the actual process that you go through, and we use that... Every term we do one inquiry. And it lasts six weeks, or seven or eight weeks even. And it's very deeply embedded now. (Lead teacher, CoP B)

Routinising was initially a conscious process, but the new way now started to gradually enter into unconscious routines.

I reflect with the children at the end of the day... It's not until we start talking about these things [that I remember]! It's probably a habit now...the kids are so used to doing evaluations and thinking about what they did, and how they can get better and improve...I suppose we're reflecting all the time at the moment, putting in these new tools and teaching ideas." (Teacher, CoP B)

This interview excerpt demonstrates the normalisation and internalisation of knowledge: the teacher's use of thinking tools has become so thoroughly integrated into their daily routine that it has become tacit; or unconscious. The teachers' use of thinking tools is not easily recollected because these tools are part of the 'normal' teaching repertoire. For this teacher and others in CoPs $\mathrm{A}$ and $\mathrm{B}$, knowledge of the new way became so strongly personalised and owned during Settling that it was no longer about specific models, practices or theories, but simply about how to teach. 
The new way had also infused teachers' interpretive frameworks, influencing their understanding of their role:

It's about ways that I can actually encourage the children...so that they can be out there using the equipment, and bringing it back and sharing it with the classroom, and [me] being more a facilitator, rather than... taking the photos myself... It's about the children, and then learning, and then being in charge. The children need to come first...For me, that's what it's about " (Teacher, CoP A)

The new way was also strongly internalised; bound up with people's identities and beliefs:

It's knowledge....about what I can do, what I actually know and I could do but I don't. (Teacher, CoP A)

I think it becomes the embedded belief that you have yourself about something, so it adds to you." (Principal. CoP A)

The degree of embedding that had, by now, occurred becomes clear when these statements are compared with a comment from an early online forum: "I am consciously trying to include some ICT skills in as many curriculum areas as possible."

\subsubsection{Unconscious competence?}

Three of the teachers whom I interviewed had difficulty describing what they were doing differently now compared to before the ICT PD programme. For example, one teacher described internalised knowledge almost as if it was a toolkit kept in the garage:

There's just...such a range of things that you can't really... I don't want to think about it too deeply now.

[I] But is it there?

Yes, it's all there"

[I] How would you describe where it is now?

(Laughs) It's waiting to be called on. Like for me, l've got such a poor memory, I don't remember all the specifics. I remember what I need to remember at the time, and if I want to go back to it then I've got those readings.

It seemed that these teachers may have reached a stage of unconscious competence. ${ }^{19}$ They had been active participants in their cluster's early online forums,

\footnotetext{
${ }^{19}$ Unconscious competence is the final stage in a well-known, anonymous four-quadrant model of competency development.
} 
but at the time of interviews, these forums had been inactive for six months, and they did not interact with the blogging community. I wondered whether their recent lack of an incentive to reflect on their practice online had contributed to their level of unconsciousness. However, their difficulty in recall may have been to do with other issues, such as their ability to focus at the time of interviews. (One of them was pregnant.)

Internalised knowledge could be seen as being the aim of KT, but if knowledge is embedded so deeply that it is no longer critically reflected on, it risks becoming stale. A change agent in CoP D who had witnessed a period of prolonged institutionalisation warned that:

One of the things which makes things stick has been this process of institutionalising, but as you institutionalise, you kill the community. There's a tension there, or perhaps the passion runs out. The vision becomes harder to sustain.

This theme was strongly supported through the participant check. As one participant eloquently noted on the anonymous feedback wiki:

Your model is a great way for us to stop and consider the journey we have been on and look at where we are going. The Settling stage is an important stage but I think if we settle there for too long we will possibly start to sink...

\subsubsection{Reviewing the journey}

During Settling school leaders engaged in reviewing the journey, celebrating the gains made by going over them in staff meetings and presentations. This involved teachers in running show-and-tell sessions, providing a sense of payoff intended to sustain motivation.

People won't do things unless there's a pay-off... it could be connecting with other people, it could be people acknowledging the fact that they've got something to share. It's a real buzz when someone says, "Hey, that's a good idea". Otherwise, I think it's just requirement stuff, and as soon as the requirement goes away, people will stop doing it.

It was clear from the interviews that principals were more easily able to see the positive gains made, and the extent of change, than change agents who had been closer to the action. This can be attributed a combination of factors: a disparity in visions - the change agents typically sought faster, more radical change than principals, and the differing nature of their roles. Principals had a unique perspective, and sense of time, 
that allowed them to 'see' change happen, but this did not match the perspective of those on the ground. It was therefore important for principals to acknowledge and validate the influence of the work done by lead teachers and facilitators.

Reviewing the journey helped schools to see that online communities had played a part in embedding the new knowledge:

A lot of what we've come through with is sort of a combination of ideas from the [online forum and] readings; we've sort of spinned it out, and adapted it to where we're going. (Principal, CoP A)

Despite this acknowledgement, most principals seemed unaware of the real impact of online CoP; particularly their 'invisible' dimension.

\subsubsection{Summary}

During the Settling stage, knowledge of the new way became more broadly and deeply embedded in schools and teachers' routines and identities. The new approaches were now being used routinely and were strongly owned. They had been adapted and customised by schools and personalised and internalised by individuals. Teachers had modified both their practice and their interpretive frameworks (how they thought about teaching), to become more compatible with the new way. Online CoPs played a limited part in this stage per se, but much of what occurred can be seen as an outcome of earlier online CoP activities. In particular, the new emerging strategic documents represented the flowering of the once-challenging ideas that had been seeded in online forums, and had subsequently infused their way into the workplace, together with the new culture of professional dialogue. 


\subsubsection{Continuing the journey}

Settling did not mark the end of the knowledge embedding process. Those who attended participant check workshops emphasised a view that that additional embedding cycles were needed in order to achieve deep, sustained professional change at school level. They saw it as important to provide sufficient time for new ways of working to become embedded, but felt that settling for too long could lead to stagnation, with routines replacing thinking. They identified strongly with my cyclic model and stressed the importance of schools Continuing the Journey - embarking on a new KEC that was consistent with the new paradigm and that would reinforce the previous cycle. The meta-model for the embedding of transformative professional knowledge at meso-level is therefore a knowledge embedding spiral in which each successive cycle wraps around and reinforces the previous cycle. The embedding of knowledge is seen as a process that is never entirely complete; characterised by the ongoing deepening and enrichment of understanding and increasing personalisation of practice.

The duration of this study meant I was unable to witness the completion of secondary embedding cycles, but it was clear new cycles were being initiated in a number of schools. Analysis of the data suggested that there were two ways of continuing the journey: top-down and bottom up.

The top-down approach occurred when a school formally embarked on a new schoolwide professional development programme: Several schools had embarked on government funded literacy and/or numeracy programmes. The principal of one of these schools explained that the literacy programme was being 'related back' to the newly acquired knowledge:

There's been a big move over the last term into [literacy]; relating it back to what it was about effective teaching and learning [that we learned with ICT].... So we're getting consistency.... (Lead teacher, CoP B)

The challenge with the top-down approach was how to leverage the new programme in such a way that it would align with and further embed the new way. Doing this required effort on the teacher's part.

The tricky thing now is that, as we come out of that [ICT] environment [to focus on] other curriculum areas, how to put it into that way of doing things. Because ICT lends itself to hands-on and group stuff, whereas... some other curriculum areas, perhaps don't so much. But it's stuck with me...the importance of the kids being involved in what you're doing and 
what they're doing; understanding what they're doing...It's the same thing with Maths... (Lead teacher, CoP A)

In contrast, the bottom-up approach to continuing the journey relied on the energies of individual change agents to set their sights on new goals and bring others on board.

They were in part motivated by a desire to collect those teachers who had "missed the boat" the first time. One person noted, on the feedback wiki:

Continuing the journey is where I'm at. As I sail around plotting the course, staying on course, anchoring and settling, I try to extend my sails to catch others and encourage them to join in. There are many here too...that have "missed the boat". This is why this continuing the journey is such an integral part of any learning journey, so [they] are presented with many opportunities to jump on board - with support along the way from those who are plotting their course again. Of course, not all will take those opportunities; hence the need for continual encouragement to come on board.

Unlike the first KEC, which was initiated and sanctioned at the organisational level, bottom-up KECs were smaller cycles, involving groups of ready and willing participants. They had manageable foci that were synergistic with the new paradigm. For example, teachers in one school had embarked on student-led podcasting. A change agent at another school was focusing on student e-portfolios; encouraging colleagues to come on board:

I put it up on our cluster web site as a forum, and I went searching; I put all this stuff [about e-portfolios] there, which is quite a huge step. I've now posted things onto the site and some other people have started to contribute, which is really good. So there's an on-line discussion in its infancy.

Change agents had both a passion for change and a sense of social responsibility: "We all have a responsibly in our schools to 'infect' our students and staff with the desire for this radical change." They also saw themselves as providing valuable seeds to action:

To use ICT effectively in a way that... enhances learning takes creative ideas. We need to have ideas and suggestions. I find teachers usually latch onto these and then start to bring their own creativity and thoughts to bear, come up with twists that suit them, come up with other ideas and alternatives. These ideas act like seeds to actions and to new ideas, but the seeds are needed... (Lead teacher, CoP B, blog)

If a whole school was to come on board, the principal also needed to be convinced. Principals were guided by their sense of what would fit the school and what would 
work. Choosing the right focus for a school-wide initiative was vital. One principal saw this as identifying the spark(s) that would generate success.

I have to see that maybe it can have some sort of benefit or impact on the school...l'm looking and saying, "Will this fit into our school?" because...I know, fairly clearly, what direction the school's going in, and the needs that it has. And if I see that it has a benefit, then it's a spark that starts something off.... Our staff are pretty determined people, and if they see something that they see that will work, wow, I don't need to do anything. They will make it work. If I see that there's no spark there, then I just back off and have another look at it, and think, "Well, is it worth it?" And [if] it's not going to work for our school do I need to come from a different direction? (Principal, CoP A)

Change agents therefore needed to work alongside principals in planning the new focus. While supporting their colleagues, they needed to look ahead, and strategise:

It's almost as if you are plotting, in all senses of the word. Your head is up there, spinning around...it's almost schizophrenic: l've got to be ahead of the others, plotting the next strategy....

Given the duration of my study, it was impossible for me to know how successful schools would be in continuing the journey, and how the activities, issues, moderators and factors involved would differ in a second cycle. (It seems likely, for example, that Setting Out and Staying on Course would present considerably less of a challenge; suggesting the potential for shorter, more frequent cycles.) This acknowledged limitation of the model is offset to a degree by faith in the experience of my participants.

Similarly, it is unclear what role online CoPs would play throughout subsequent cycles. While top-down programmes were designed and funded to support a formal wholeschool-community approach, bottom-up initiatives seemed to be reliant on the emerging distributed, blog-centric online CoP infrastructure, and on the change agents' critical roles as connector-leaders in CoP E. Chapter 8 explains how this infrastructure helped sustain knowledge embedding at macro, or system, level.

\subsubsection{The KEC as a driver of the five embedding processes}

Throughout this chapter, while explaining the KEC, I have made references to how activities undertaken at different stages facilitated the five fundamental embedding processes, focusing, persuading, aligning, adapting and owning; providing supporting examples. The relationship between the KEC activities and the fundamental processes 
was complex, in that activities typically contributed to more than one of the key processes. I include two tables showing these relationships in Appendix G.

\subsection{Chapter summary}

This chapter has shown that embedding of the new way in schools was a gradual, complex process. It comprised six stages linked with different issues, and requiring different solutions. The KEC has provided a framework for explaining this process and examining the role played by online CoPs played in facilitating it.

Online CoPs played a changing role over time. In the early stages, facilitated, platformcentric CoPs played a critical role in bringing staff on board, building common ground and fostering new communication norms and a professional agenda that spilled over into the workplace. Online forums drove focusing and facilitated the mutual persuading and aligning that fostered the owning and normalising of change. This was further fostered by invisible online discussions amongst the change agent sub-community.

As schools and teachers began adapting, their knowledge needs became more specific and diverse. The official community became a repository, while a distributed, blog-centric infrastructure developed to support day-today knowledge needs. The change-agent sub-community became part of a larger community of passion (CoP E) and network, and their roles as brokers of knowledge became more important. Buddying and leader-follower relationships helped to drive focusing. persuading, adapting and aligning as knowledge became more customised, personalised and owned.

The KEC is synergistic with research that positions organisational KT as a staged process (e.g., Szulanski, 1996); however, there is an important difference in that the KEC is not linear, but cyclic. This arises from this study's context of transformative change. As noted in Chapter 3 , embedding a new paradigm is known to be difficult, requiring sustained change in people's belief structures and interpretive frameworks. This underlines the rationale for the spiral model.

My analysis could have stopped at this, most visible, set of results, but data coding showed that additional embedding forces were at work. A further factor fuelled my interpretative persistence: In parallel with the start of this project, I had considered the issue of relevance in IS research (Cranefield \& Yoong, 2007c). This had led me to an 
awareness of, and concern about, an apparent meso level bias in IS: For example, based on an analysis of leading IS journals, Crowston and Myers (2004) found that most research was conducted at the organisational level of analysis $(51 \%)$, followed by the individual (21\%) and group (11\%) levels (ibid). It is possible that my awareness of this bias - or my bias about it - contributed to my theoretical sensitivity. Had it not been for this awareness, I may have stopped my analysis at the meso level. However, for a combination of reasons, I was not happy to do this, preferring to consider more of the richness and complexity that emerged from my study. This approach was fully compatible with the interpretivist paradigm.

In the next two chapters I outline my findings about how online CoPs facilitated knowledge embedding at the level of the individual (micro-level) and the system of communities (macro-level). 


\section{The micro-level facilitating mechanism: Crossings}

\subsection{Introduction}

In this chapter I discuss my micro-level theory for how online CoPs facilitated embedding of the new way at a personal, individual level. Members of the CoPs in my study interacted within a complex socio-technological system made up of multiple engagement spaces; differentiated contexts within, and across which they communicated and reflected. Newly introduced online engagement spaces supplemented and complemented traditional professional engagement spaces, such as the classroom and staffroom. In combination, they created a new arena for professional interaction, characterised by polycontextuality (Engeström, et al., 1995; Goodwin, 1990; Reder, 1993); multiple parallel activity contexts or micro-worlds. Each engagement space had a distinct culture of use and distinct benefits. Embedding of knowledge was facilitated by individuals' ongoing crossings between them. As they continually crossed between different engagement spaces, they had to recontextualise and personalise knowledge. This facilitated deeper understanding and owning of ideas. Crossings occurred within a social system that favoured the alignment and convergence of interpretive frameworks and beliefs, further facilitating embedding of the new way.

The facilitating mechanism outlined here worked like a catalyst of embedding for active members of the online CoPs; notably those in the change agent sub-community, who used a wide range of online tools, but also those who used a smaller range of tools, such as IM, online forums and email. (The participant group comprised mainly active individuals; selected because of my how research question.) In order to understand how online CoPs contributed, indirectly, to embedding of knowledge amongst those who were inactive in online settings, it is necessary to understand the macro-level knowledge embedding mechanism outlined in Chapter 8.

In this chapter, I firstly introduce the complex CoP environment, explaining how CoP members used, and were influenced by, engagement spaces. I then examine the process of crossings in depth, and how they occurred across various dualities of context. In particular, I consider theory-practice crossings and how they facilitated embedding of knowledge through personalisation. Drawing on the work of cognitive scientists, I examine the significance of polycontextuality in relationship to the 
embedding of knowledge. I then summarise the relationship between crossings and the five knowledge embedding processes.

\subsection{Complex CoP system with multiple engagement spaces}

In order to understand the embedding-facilitating mechanism at the micro level, it is necessary to consider the nature of the CoP environment within which the active online CoP participants in this study interacted..$^{20}$ This was not a traditional platform-based community, but a more complex system, comprising diverse communication settings linked by individuals and themes of common interest. It was an example of Castro's (2004, 2006) Online CoP Ecosystem, a setting in which overlapping groups deal with the same subject and individuals participate in more than one online setting, with different roles and degrees of activity.

The most active online CoP participants (lead teachers and unofficial change agents) interacted via a range of online tools, including online forums, IM tools (Skype or ichat), RSS feeds, Twitter, Del.icio.us (social bookmarking) and, in the case of very active members, voice-threads, podcasts, Teacher Tube videos, Second Life and/or other Web 2.0 tools. These different tools supported different needs and were used in complementary ways (see figure 30 ). For example, IM provided a ready means of seeking technical help in the daytime and gaining feedback on lesson planning in the evenings. Online forums facilitated personal reflection, while providing visibility to colleagues' beliefs, thoughts, problems, and evolving practices. This, in turn, created entry points for face-to-face staffroom dialogue, which fostered new relationships that could be leveraged via IM after-hours. Informal discussions, via IM and in the workplace, were used to persuade members to contribute to forums or blogs, to test out emerging ideas, and to gain buy-in from peers prior to finalising forum postings.

It is useful to view this rich environment as being made up of multiple engagement spaces - diverse communication and sense-making contexts, creating a situation of polycontextuality. As well being associated with the use of different tools, each engagement space had its own set of participants and its own culture of use: implicit rules governing the nature of the agenda, the genre (language, tone and style) and the conventions of communication.

\footnotetext{
${ }^{20}$ One of the five communities had completed the professional development programme over two years prior to data gathering and had entered into a period of inactivity as an online community.
} 

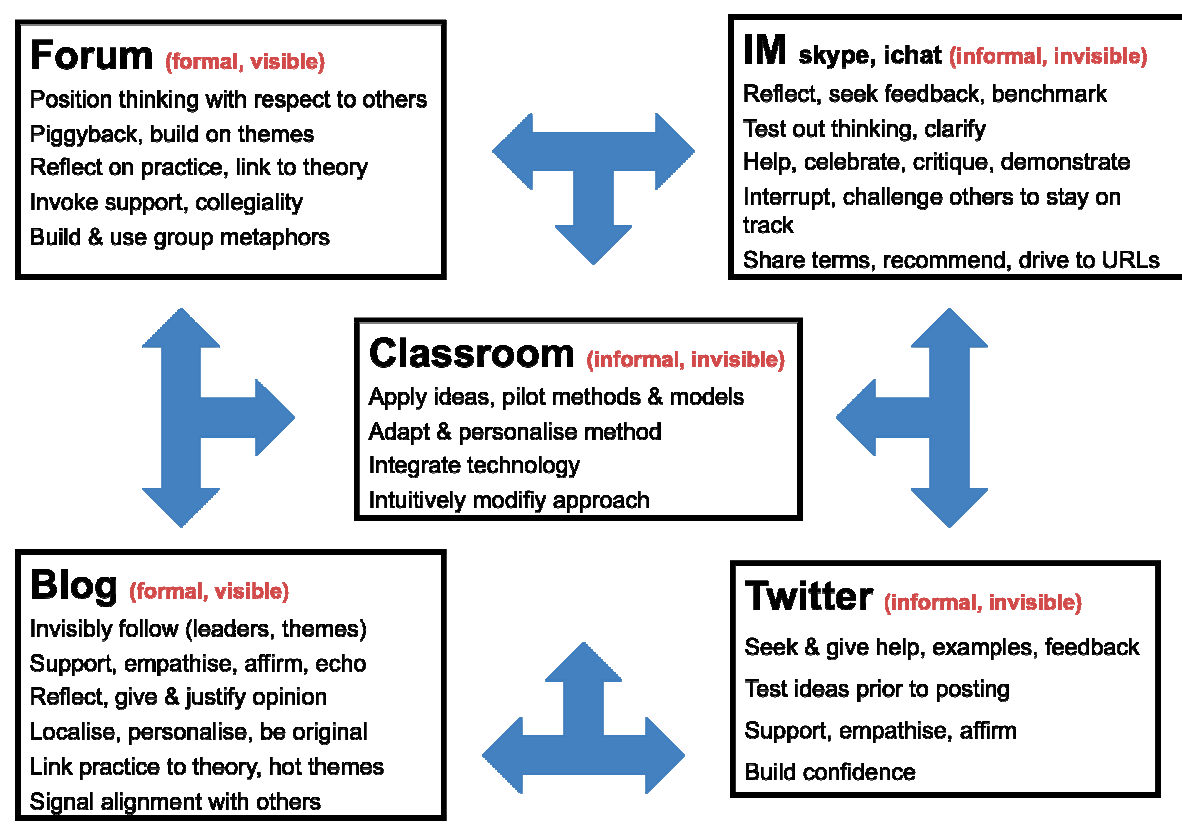

Figure 30: Polycontextuality - amplification of the professional environment with diverse engagement spaces, providing complementary fuctions.

For example, blogs were a semi-formal, but highly personalised, genre in which individuals were expected to cite one another, voice their opinions, and share their stories in ways that demonstrated learning. Blogging culture placed a high value on the contribution of new perspectives on themes of shared interest, examples of novel practice, and inspirational content that reinforced the community's belief in the potential of (effectively used) technology to inspire student learning.

In contrast, Twitter was a highly informal text-messaging tool, used by individuals to post brief text-based 'tweets' (updates on their day-by-day, or hour-by-hour, activities) to self-selected followers. Tweeting culture encouraged teachers to behave much less professionally; boasting about successes, complaining about challenges, and using a humorous 'low' style that contrasted strongly with their blog-based personas ( "What is it about Twitter that brings out the naughty child in us?'). Tweeting was a low-visibility, low risk activity that created powerful social allegiances that could be called on; for example, by requesting feedback on blog postings. This system of specialised contexts, conventions and genres can be seen as arising from human-technology interaction, as the community matched technological opportunities and constraints to shared and individual needs. 
Figure 30 shows the complementary ways in which online engagement spaces were used in relationship to teachers' emerging practice in the classroom. Interaction in different engagement spaces often resulted in mutually reinforcing outcomes. For example, in the Coming on Board stage, schools held meetings to discuss challenging content and collaboratively workshop their forum responses. This reduced individuals' barriers to online participation while securing conceptual buy-in, and sowed the seed of a common vocabulary that was embedded through subsequent online dialogue, promoting intra-school alignment. Prior to exposing their individual ideas in forums or blogs, teachers rehearsed their thinking in collegial contexts, face-to-face and/or via IM. The testing out of emerging thinking in a safe (invisible) online context helped people to articulate their ideas, and improve and validate their thinking, through the input and implicit approval of respected peers. These activities, resulting in the appearance of well-crafted thinking in online forums, drove the mutual alignment of teachers' interpretive frameworks. It increased their ownership of, and confidence in, their personal opinions about the new way and shored up their belief in the direction of change.

It was being able to get ideas and opinions from other teachers at other schools...that validates [your] ideas (Lead teacher, CoP A)

Figure 31 shows how this new polycontextual system had dramatically expanded on the capabilities of the traditional system in terms of opportunities for professional engagement. (The diagram simplifies the situation, illustrating commonly used engagement spaces, but excluding meetings, phone calls and SMS.) Traditional spaces in which teachers engaged with their profession appear as dark circles (the staffroom, workshops, classroom, home and email). The new online engagement spaces are shown in a pale colour (forums, blogs, IM and twitter). Lines show where crossings occurred between online and offline engagement spaces. (The dotted lines indicate less common crossings.) The engagement spaces are plotted on a continuum showing how they span a spectrum from public to private and visible to invisible. (I have viewed forums as public in relationship to the CoP because all teachers in each CoP were automatically signed up as members. Blogs were public in the common sense of the word.)

What is notable in this diagram is the fact that teachers' traditionally private, solo engagement spaces - the classroom (where they teach) and the home (where they plan and reflect) - were made less private through the availability of IM, forums, blogs 
and Twitter. Teachers could readily connect with others in their CoP during their solo, reflective times at home by crossing into, and between, these engagement spaces.

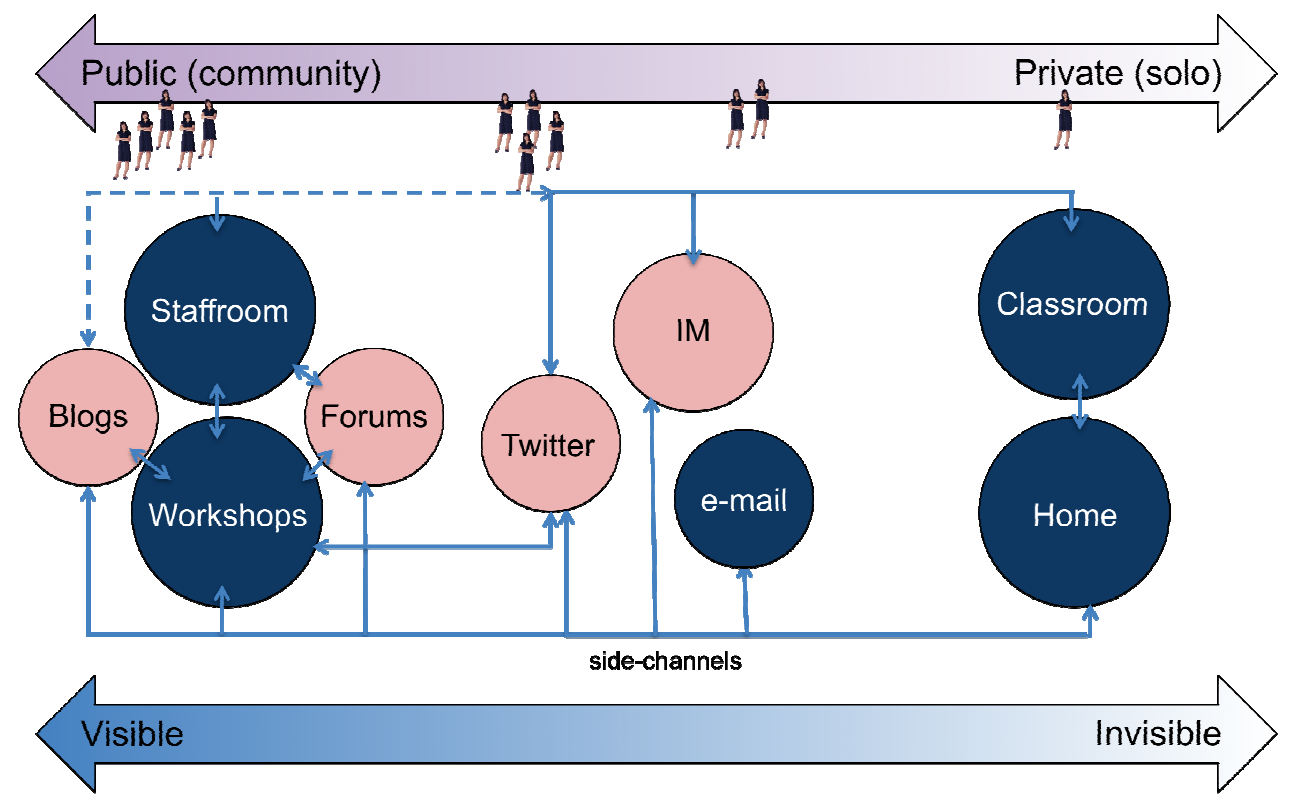

Figure 31: Engagement spaces, showing points where crossings were made. New online engagement spaces reduce the isolation and increase the visibility of individuals.

\subsection{Crossings as a driver of knowledge embedding}

Analysis of communication records, backed by the participant check process, revealed that crossings were a key driver of knowledge embedding at the individual level.

Crossing engagement spaces involved crossing between different sub-communities, different roles and personas, different literary genres and social conventions, as well as between synchronous and asynchronous modes of communication, online and offline contexts, and the work and home environments; often simultaneously. The system of engagement spaces can be seen as providing a series of contextual dualities that needed to be continually negotiated by individuals: online/offline, visible/invisible, public/private formal/informal, reflective/active (theoretical/practical), leader/learner, and home/work (see figure 32).

As individuals repeatedly crossed between online and offline, formal and informal, public and private, reflective and active, and visible and invisible engagement spaces, they needed to adapt their communication approach to fit the different genres, conventions and audiences associated with each setting. This involved them in an ongoing recontextualisation process, requiring them to translate, interpret, adapt, combine, argue, justify and re-articulate ideas. 


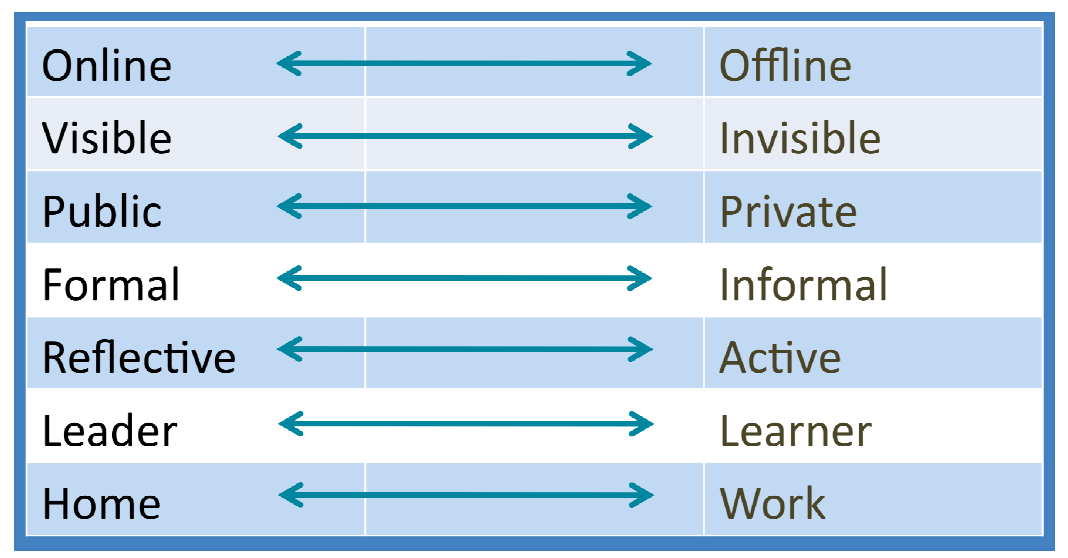

Figure 32: The multiple dualities of context provided by the system of engagement spaces

For example, a teacher's blog posting might draw on an idea stated in an online forum, but there were community pressures (linked with the blogging culture of use) for the idea to be more expansive, more argumentative in structure, and more authoritative (backed with examples) in this new context. Supporting examples could be gathered from colleagues, via an IM discussion, or a Twitter exchange. The effort involved in this recontextualisation process was significant: The challenge in creating a blog or forum posting was less about how to articulate one's thinking than working out what one's thinking was, and how it related to the views of others. This process of recontextualisation through crossings served to clarify and deepen personal understandings:

My learning is a dialogue. I read, comment, cross-comment, post, hyperlink and think and link. The views of others influence my thinking through this dialogue I have to re-define my thinking - by justifying why I think the way I do I clarify in my own head what it is that I do actually believe. (Anonymous, wiki)

Crossings also occurred in real time. In one example, a facilitator intervened into a teacher's classroom via IM, challenging them about a forum posting (see Table 14, example (e)). Another teacher reported engaging in a Twitter-based dialogue with their CoP E peers during conference keynote speeches. By using a collaborative engagement space as a synchronous side-channel, these people had been able to interpret, contextualise, validate, and enrich speeches on the fly, through a joint critiquing process:

After I... began to have more contact with people within the conference via twitters it changed the dimension of the conference. It changed from being...thoughts between the speaker and myself to the possibility of 
having other people's opinions...the twitters and examples that were given in rebuttal or agreement... It meant that I was questioning and thinking during the keynote to a higher dimension than if I was just sitting there listening to HER. (Teacher, blog, CoP E)

This was an example of a visible/invisible crossing that built personal understanding while aligning it with community norms: By crossing from the shared, visible (and audible) conference presentation to a parallel engagement space that was invisible to most conference participants, the teacher gained access to a commentary that simultaneously improved their understanding and aligned it with those of others from the online CoP. Visible/invisible crossings were a powerful means of aligning ideas, and occurred in other contexts; for example, when teachers used IM to test out ideas for forum postings or Twitter to solicit affirmative feedback on blog posts.

I will now consider theory/practice crossings, which contributed particularly powerfully to embedding of the new way. I have already mentioned the matching up of theory and practice that occurred in the middle stages of the KEC. The concept of crossings allows me to examine this theme more deeply, from an individual perspective.

\subsection{Theory-Practice Crossings}

It sort of firms up what you believe, and then you look at what you're doing, and so you change that...." (Lead teacher, CoP A)

Teachers' efforts to integrate and reconcile the theoretical and practical dimensions of practice are seen as critical to development of their personal professional knowledge (Bromme and Tillema 1995; Leinhardt et al. 1995). Similarly, Wenger (1998) views professional learning in a CoP as involving a continual tension between the duality of participation in work and reification (documented work-related knowledge). This theme from the literature was strongly reinforced by my findings.

As the ICT PD programme progressed, active online CoP members found themselves engaging in an increased number of theory-practice crossings (crossings between reflective and active contexts). This was driven by their participation in an increased number of engagement spaces suitable for - and where cultures of use demanded - 
reflection. This led to deeper, more personal, understanding of the new way by driving an iterative mutual alignment between their practice and their emerging beliefs:

It sort of firms up what you believe, and then you look at what you're doing, and so you change that.... (Lead Teacher, CoP A)

Firming up of beliefs was significant to the embedding process: Research has indicated that in order for professional change to be sustained, teachers' new knowledge and practices must be underpinned by compatible beliefs and interpretive frameworks (Handal, 2004; Keys, 2006; Richardson \& Placier, 2001).

Some teachers used IM and Twitter for spontaneous real-time discussions about the theory-practice relationship, but forums, wikis and blogs provided more formal loci for this purpose. These engagement spaces created a written record, creating an incentive for deeper thinking and more careful articulation of ideas. In addition, the cultures of use associated with forums, wikis and blogs strongly encouraged reflection and the relating of one's practice to the theories of others. The 'theories' with which teachers engaged were typically the personalised, persuasive theories of thought leaders. While being consistent with the new paradigm, and apparently aligned with research into effective teaching, they were non-academic in nature, based around simple, strongly memorable themes (see section 7.6).

My analysis of online records revealed a complex tapestry of theory-practice crossings. I provide five different examples in table 14. In each case, the teacher concerned recalls an incident in which they juxtaposed theory to do with the new way with their teaching practice. In the course of recounting this event, they articulate and expand on it, considering its meaning to them as teachers. The reflected-on events can be seen as having gained weight in terms of personal meaning, when compared with the initial thought or event.

Teachers most often reflected retrospectively on their teaching practice in light of theory (see table 14, examples (a) and (c)). Sometimes they also reflected on spontaneous theory-practice connections that they had made in the heat of the classroom. These stories illustrated memorable reflection-in-action episodes (Schön, 1987) that had moved them forward in their understanding, adaptation, and application of theoretical knowledge. 


\begin{tabular}{|c|c|c|c|}
\hline Nature of Crossing & Example & Excerpt & $\begin{array}{l}\text { Contexts } \\
\text { Crossed }\end{array}$ \\
\hline $\begin{array}{l}\text { (a) Reflection on } \\
\text { practice } \\
\text { (in light of theory) }\end{array}$ & $\begin{array}{l}\text { Teacher reflects on and reframes } \\
\text { their practice in an online forum, } \\
\text { adapting Prensky's metaphor of } \\
\text { the teacher as digital immigrant. }\end{array}$ & $\begin{array}{l}\text { "As I read the article I found myself } \\
\text { (wondering) what language I use with my } \\
\text { class... do I speak the language of the pre- } \\
\text { digital age because of the fact that all of my } \\
\text { teachers did?... I will be more aware of this } \\
\text { from now on". }\end{array}$ & Forum/Classroom \\
\hline $\begin{array}{l}\text { (b) Reflection in } \\
\text { practice } \\
\text { (in light of theory) }\end{array}$ & $\begin{array}{l}\text { After reflecting on a keynote } \\
\text { speaker's theory in a blog, } \\
\text { teacher makes a spontaneous } \\
\text { adjustment to practice, adapting } \\
\text { the theme of teacher as game } \\
\text { master, later reflecting on the } \\
\text { event on the blog, enfolding it in } \\
\text { the theory. }\end{array}$ & $\begin{array}{l}\text { "Well it actually worked, I can't really } \\
\text { believe it ... (He) was taking about the } \\
\text { LEVEL BOSS at the end of video games } \\
\text {... Here's what I did... A couple came to me } \\
\text { and said- "Haven't got any mistakes"... } \\
\text { What's going on here I think. So I stand up } \\
\text { and address the class. I tell them... Writing } \\
\text { is like a game... I said the level boss needs } \\
\text { to be blasted and your only ammo are the } \\
\text { words you are trying...I am now the Games } \\
\text { Master and they possess a cheat book...." }\end{array}$ & $\begin{array}{l}\text { Conference/Blog/ } \\
\text { Classroom/Blog }\end{array}$ \\
\hline $\begin{array}{l}\text { (c) Reflection on } \\
\text { practice } \\
\text { (in light of challenge to } \\
\text { elicit theory) }\end{array}$ & $\begin{array}{l}\text { Teacher reflects on the need to } \\
\text { justify their ICT-based practice in } \\
\text { light of theory, in response to a } \\
\text { collegial challenge made at } \\
\text { lunchtime. This highlights an } \\
\text { unresolved underlying issue, } \\
\text { raising its profile and extending } \\
\text { its life. }\end{array}$ & $\begin{array}{l}\text { "..it suddenly occurred to me that I really } \\
\text { needed to find out why this way of learning } \\
\text { and teaching felt so right and it felt } \\
\text { necessary, almost urgent for me to redefine } \\
\text { my own personal pedagogy to ensure that I } \\
\text { wasn't just bombarding my students with } \\
\text { the "bells, and whizzy stuff" available on } \\
\text { the "net" simply because it felt right or } \\
\text { because I liked it. I'm also feeling an } \\
\text { increasing need to be able to justify why } \\
\text { "that's what we do 'round here"... }\end{array}$ & $\begin{array}{l}\text { Face-to-face } \\
\text { discussion/Blog }\end{array}$ \\
\hline $\begin{array}{l}\text { (d) Reflection on } \\
\text { reflection on } \\
\text { theory }\end{array}$ & $\begin{array}{l}\text { Teacher recounts an earlier } \\
\text { spontaneous reflection that } \\
\text { occurred while cycling, capturing } \\
\text { and extending the recollected } \\
\text { thoughts. }\end{array}$ & $\begin{array}{l}\text { "I was reading David Warlick's School } 2.0 \\
\text { blog yesterday and as I cycled through the } \\
\text { streets... I began to give it some more } \\
\text { thought." }\end{array}$ & $\begin{array}{l}\text { Blog/Cycling/ } \\
\text { Home/Blog }\end{array}$ \\
\hline $\begin{array}{l}\text { (e) Intervention in } \\
\text { practice } \\
\text { (to facilitate live } \\
\text { application of theory) }\end{array}$ & $\begin{array}{l}\text { In response to a teacher's forum } \\
\text { comment about the limitations } \\
\text { of clipart, a facilitator uses IM for } \\
\text { an unscheduled intervention into } \\
\text { their classroom teaching, } \\
\text { prompting the teacher to engage } \\
\text { students in a more constructivist } \\
\text { approach to the use of the tool, } \\
\text { as had originally been promoted } \\
\text { in the forum. }\end{array}$ & $\begin{array}{l}\text { "I saw she was on the I-chat, and I asked } \\
\text { her, "Why do you do that and what do you } \\
\text { mean?" And she was saying, "Well, if I } \\
\text { want them to draw a camel, they've never } \\
\text { seen a camel, how do they know what a } \\
\text { camel's like unless I show them a } \\
\text { picture?"...And so she was then talking to } \\
\text { her kids... saying, "Mrs. French (the } \\
\text { facilitator) wants to know about the } \\
\text { camels. What are the things that camels } \\
\text { have?"'" }\end{array}$ & $\begin{array}{l}\text { Forum/IM/ } \\
\text { Classroom }\end{array}$ \\
\hline
\end{tabular}

Table 14: Theory-practice crossings: examples

For example, in table 14, example (b), a teacher, 'John', blogs about how he redirected his teaching approach following an aha moment: Realising that his students were unmotivated, he saw an opportunity to apply a concept on which he had earlier reflected - the teacher as game-master. Improvising with a video game analogy, John repositioned the lesson as a competitive game and the students engaged. By retelling the event and illustrating its success on his blog, John affirms and reinforces his learning. Rather than dropping the ball - as could happen in the absence of reflection he continues the process of adapting, amplifying the meaning of the event in retrospect by aligning his improvisation with the source theory to analyse why it worked. The 
blogging culture of use requires John to attribute the game master idea to its originator, David Warlick. This ensures focusing: John must reconnect with the metaphor's intent and underlying theory. The conceptual effort involved in this theory-practice crossing, plus the impact of the self-affirmation (persuading) more deeply embeds the meaning and significance of the event, increasing the likelihood that it will impact on John's ongoing practice. Furthermore, by tagging his blog post with Warlick's name (using Del.icio.us), John increases the chance of receiving further affirmative feedback from like-minded people (fans of Warlick who use RSS feeds).

Theory-practice crossings could be sustained over time, traversing multiple engagement spaces as teachers' understanding and practice evolved. An example of such a sequence of crossings is provided in Appendix $\mathrm{H}$, tracing a teacher's progressive introduction of a role-play method of inquiry learning, from 2007-2008.

IM was occasionally used for real-time theory-practice interventions, with individuals interrupting their colleagues' teaching for unscheduled, invisible in-class mentoring (see table 1, example (e)). This remarkable departure from the closed-door teaching tradition illustrates the potential for collaborative processes in the course of professional change. Interventions were made possible by a shared history of engagement on a theoretical topic, the resulting visibility of personal knowledge constructs, the relationship of trust that had been built from social online activity, and a culture of openness in relationship to the context of IM use.

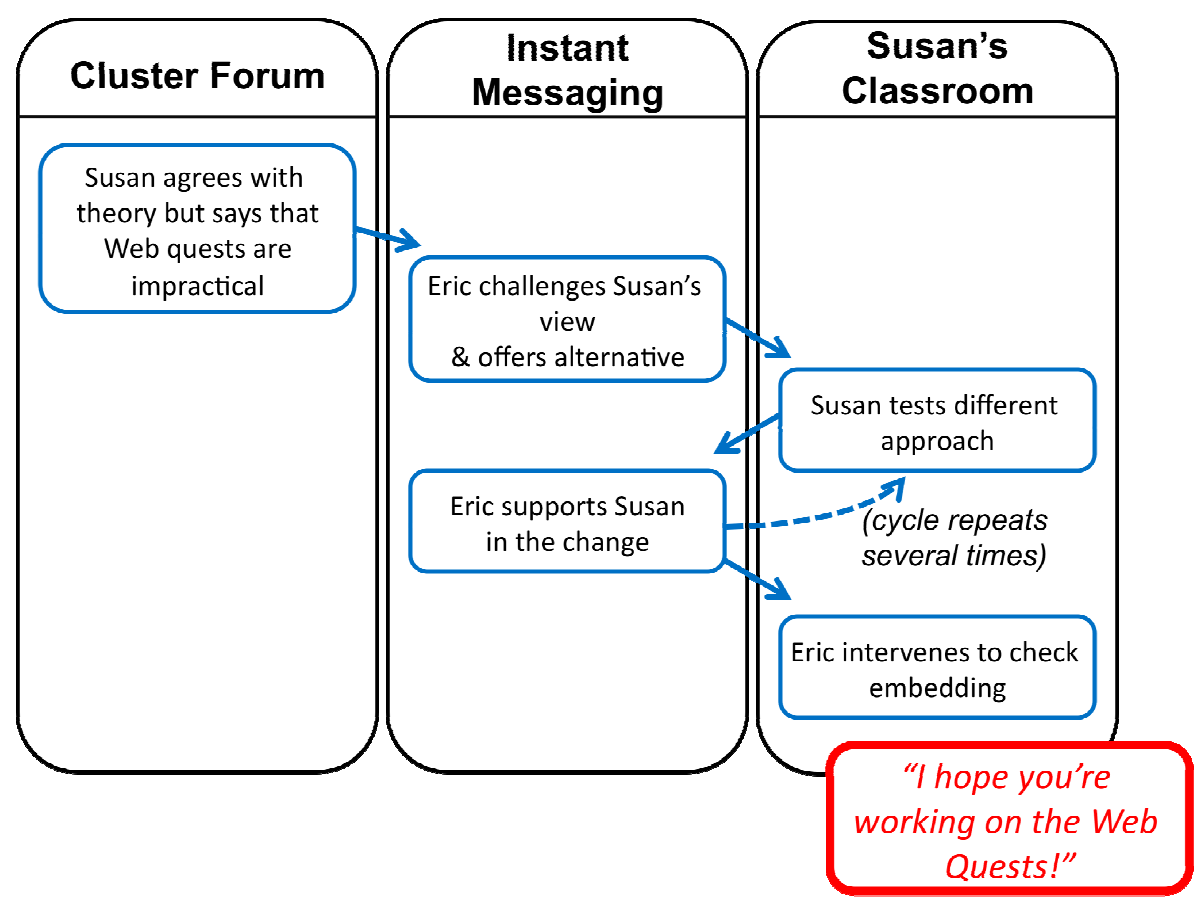

Figure 33: Making WebQuests practical - theory-practice and visible-invisible crossings between buddies 
Another interventional theory-practice crossing is shown in figure 33. (This is also both a visible-invisible and public-private crossing. I have discussed it in Chapter 6 as an example of buddying.) In an online forum, 'Susan', expresses frustration with using WebQuests. Crossing to an invisible side-channel - IM - 'Eric', a teacher from another school, challenges her to persevere. He suggests adapting the method to make it more practical. Susan tries out his suggestion in class, engaging in further IM discussions with Eric about the method's practicalities and intent, as she iteratively adapts it for her own purposes. Finally, Eric invisibly intervenes into Susan's class in real time and jokes, via IM, "I hope you're working on the WebQuests".

The image that emerged from the different examples of theory-practice crossings was one of two parallel ladders (theory and practice), with the practitioner crossing repeatedly between them, as they climbed upwards, gradually refining and personalising their understanding through a process of aligning the theoretical and practical dimensions of their knowledge, while taking on board the ideas of others.

It was looking at the theory of teaching, and the practice.... the theory backed up what you were doing practically, and if you were doing something practically, you could often match it up with something in the theory, and (say), "That's why we do that.'" (Lead teacher, CoP A)

\subsection{Polycontextuality as an enabler of embedding}

In relationship to my research focus - the embedding of professional knowledge - the feature of the CoP environment that made these crossings so powerful was its polycontextuality. Traditional workplaces are, to a degree, polycontextual in that they comprise different communication contexts, such as official meetings, coffee conversations, email dialogues etc. Goodwin (1990) describes these contexts as professional micro-worlds that provide "particular forms of access, structures that shape perceptions and talk, ways of acting etc."(p. 46). Studies of expert cognition have demonstrated that polycontextuality can contribute to professional learning in situations of novelty (Engeström, et al., 1995; Goodwin, 1990; Reder, 1993). This is because, as professionals move between different activity contexts, or participation frameworks, they integrate knowledge from, and across, them, revising their knowledge in the course of doing so. The development of new professional knowledge is seen as being facilitated primarily by this movement between contexts. 
Polycontextuality can be seen as providing a more granular view of the work environment than situated learning theory (Lave \& Wenger, 1991), which has traditionally underpinned studies of CoPs. It places boundary crossing as central to personal knowledge transformation. KM research has also highlighted the importance of boundary crossing in knowledge transfer exercises (Ancona \& Caldwell, 1992; Cranefield \& Yoong, 2007b; Harada, 2003; Pawlowski \& Robey, 2004). Crossing boundaries requires the boundary spanner to filter, interpret and translate knowledge and to create mediating concepts, or boundary objects (Star, 1989), such as metaphors and models. These make ideas accessible to others, facilitating KT from one context into another.

The online CoP participants in my study can be seen as boundary spanners who continually negotiated the boundaries of engagement spaces and the associated system dualities. The translation and interpretation efforts required were driven by the contextual requirements (cultures of use) of the engagement spaces and their own need for understanding. Crossings required focusing clearly on ideas and how best to communicate them as they were recontextualised. This led to strong personalising and owning of meaning.

In CoPs $\mathrm{A}$ and $\mathrm{B}$, online participation had led to a significant expansion in the number of professional engagement spaces available, creating a situation of enhanced or hyper-polycontextuality. For active online community members, this was linked with a higher-than-usual frequency of inter-professional communication, extending well outside of working hours. By leveraging social technologies to provide new contexts for reflection on practice, this system mitigated against the barriers to reflection that exist in many traditional work environments, highlighted by Leinhardt et al. (1995) as being antagonistic to the embedding of new knowledge. The process of repeated crossings within the polycontextual system was like an engine that helped to drive the embedding of knowledge.

In the earliest study of CoPs, Lave and Wenger (1991) found that apprentices gained knowledge of their profession through situated learning in the CoP. Situated learning is seen as inseparable from the activities, context and culture within which it occurs (i.e., it is situated). The CoP members in my study were also impacted on by their context, activity and culture; but with a difference: During the course of a day and night they were situated in diverse contexts (engagement spaces) with different cultures of use. The learning that occurred - in this case the embedding of professional knowledge - 
was promoted by their ongoing movement between this multiplicity of contexts, combined with social pressures that promoted alignment at community level. This could be seen as a specialised kind of situated learning - one that occurs in a situation where there are polycontextual micro-environments. Crossings were not about immersion in any particular environment, but about the learning that arises from the crossing of boundaries between micro-environments. The individuals in this study were actively involved in constructing personal meaning in a conscious, self-reflective way. This resulted from a high number of theory-practice crossings, promoted by the increased number of engagement spaces suitable for (and demanding of) reflection, provided by the online CoP.

\subsection{Powerful content, personalisation and convergence}

The complex polycontextual landscape resulted in individuals undertaking personalised trajectories of sensemaking that differed from those of their colleagues. However, this occurred within a broader environment that favoured the convergence of knowledge and beliefs. While teachers undertook repeated crossings, the themes and threads of ideas that influenced them, and which promoted community-level convergence, spread to permeate different contexts. Thematic repetition occurred across engagement spaces, with particular themes being favoured, and more strongly reinforced, by the community.

Powerful content - memorable metaphors and motifs, typically introduced by thought leaders, such as Prensky's (2005-6) teacher as a digital immigrant, Warlick's (2007) telling a new story, and the ubiquitous teacher as learner, facilitated the convergent personalisation of interpretive frameworks as they were appropriated, varied and recontextualised by individuals in the CoP. These metaphors functioned as community boundary objects, or interpretive anchors. In the process of crossings, they had a tendency to inveigle themselves deeply into the consciousness of individuals, merging into their language and thought patterns. An example of this unconscious impact can be seen in Figures 34 and 35 below, where an individual ('Alex') twice recommends and cites an influential thought leaders' video, then later (after a number of crossings) unconsciously replicates the thought leader's themes in a blog posting. Given the strong citation protocol in this blogging community, the absence of a citation in the final posting is significant. I do not interpret this as a case of unconscious plagiarism, but rather as an indicator that Alex's ideas concerning creativity had become so strongly internalised, from repeated exposure to the original themes and their variants, that they 
had been embedded. The source themes, while recognisable, had been recombined, varied, and extended in the course of a sustained value-adding personalisation process, until they were owned by Alex.

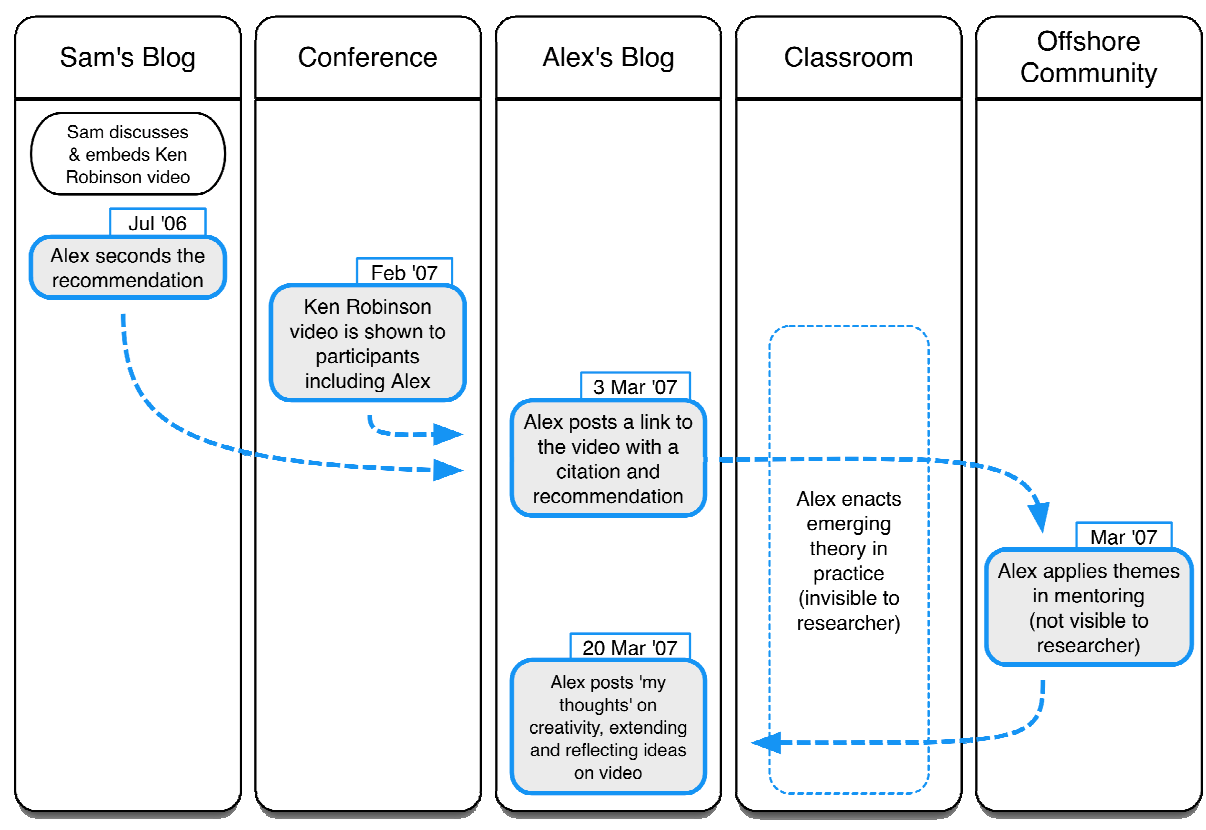

Figure 34: Embedding of memorable themes via crossings

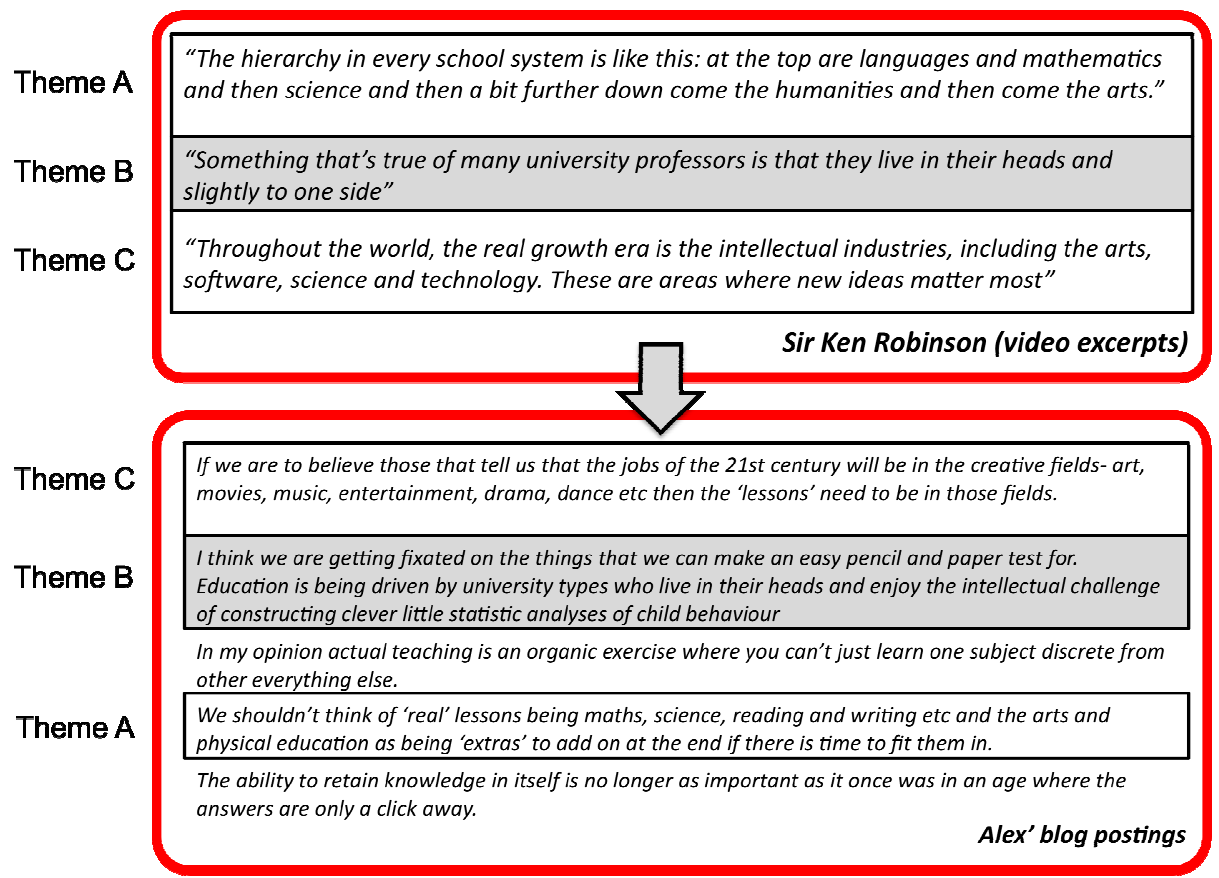

Figure 35: Embedded themes (following the above series of crossings) - source content at top 
The themes introduced by thought leaders were characterised by their memorability. The memorability of ideas can facilitate the transmission of cultural belief systems through contagion. (This is a concept underpinning epidemiological theories for the transmission of culture (Norenzayan and Atran, 2004)). This is significant here because in order for a change in professional understanding to be sustained, supporting beliefs are required (Handal, 2004; Handal and Herrington, 2003; Keys, 2006; Martin, 1993; Smith et al., 2005). These findings suggest that the themes of memorability and cultural contagion of beliefs are worthy of further investigation in relationship to knowledge transfer on online CoPs.

Figure 35 also illustrates Alex's adaptation of the source content to align more explicitly with the new way. In the cited example from her blog, she reframes the source themes by linking them with one of the subthemes of the new way: an emphasis on the learning process rather than on knowledge as a product: "The ability to retain knowledge itself is no longer as important as it once was in an age when the answers are only a click away". Alex has not simply embedded the 'source' content from Sir Ken Robinson - she has appropriated it in a way that brings its persuasive power to bear on a dominant community theme, reinforcing it, and further driving the embedding of the new paradigm. 


\subsection{Crossings and the five embedding processes}

The micro-level facilitating mechanism of crossings promoted all five of the fundamental knowledge embedding processes: persuading, focusing aligning, adapting and owning. In Table 15, I summarise how this occurred.

\begin{tabular}{|c|c|}
\hline Process & Ways in which promoted by crossings \\
\hline Persuading & $\begin{array}{l}\text { The cultures of use for blogs and forums required individuals to recontextualise ideas in a } \\
\text { persuasive way, with examples. } \\
\text { IM and twitter were used to persuade colleagues to contribute ideas in other, visible } \\
\text { engagement spaces (such as wikis, blogs and forums). } \\
\text { Persuasive themes had a contagious impact - they were appropriated by individuals and } \\
\text { taken into new engagement spaces. }\end{array}$ \\
\hline Focusing & $\begin{array}{l}\text { Theory-practice crossings involved focusing deeply on specific aspects of knowledge. } \\
\text { Justifying a stance (for a blog or forum) required focusing on an argument. }\end{array}$ \\
\hline Aligning & $\begin{array}{l}\text { Theory-practice crossings fostered gradual aligning of beliefs, interpretive frameworks } \\
\text { and practice. } \\
\text { Norms and thematic replication fostered aligning of thoughts/beliefs. }\end{array}$ \\
\hline Adapting & $\begin{array}{l}\text { Crossings involved the continuous adaptation of knowledge to contextual demands. } \\
\text { Theory-practice crossings promoted mutual adaptation of interpretive frameworks and } \\
\text { practice. }\end{array}$ \\
\hline Owning & $\begin{array}{l}\text { Crossings promoted owning of ideas through the need to adapt, differentiate and } \\
\text { personalise, and the deep engagement with ideas this required. } \\
\text { The memorable ideas of thought leaders were taken up, adapted and appropriated } \\
\text { (owned) by individual teachers. }\end{array}$ \\
\hline
\end{tabular}

Table 15: The micro-level mechanism and the five knowledge embedding processes

\subsection{Chapter summary}

The focus of this chapter has been on explaining how online CoPs facilitated the embedding of knowledge at the micro (individual) level. I have highlighted the fragmented, polycontextual nature of the complex online CoP environment as having played a key role in promoting the embedding of knowledge. In this polycontextual environment, individuals needed to undertake frequent crossings between different engagement spaces. These crossings involved them in an ongoing process of recontextualisation, so as to fit the different requirements of each communication context and a demand for originality. Crossings also helped teachers bridge the duality between theory and practice, making connections between the new way and their teaching. The reinterpretation, reconstruction, and personalisation of meaning that 
accompanied these crossings enriched their understanding while increasing their sense of ownership of knowledge, powerfully embedding it.

While individuals undertook different trajectories of crossings, they did this within an environment in which there broad convergence and alignment of knowledge and beliefs. Polycontextuality provided a setting in which influential themes were readily distributed and magnified. Memorable themes and metaphors and, as one participant put it, "threads of ideas" - were recycled and varied until they permeated many different community contexts. This, combined with social norms, promoted the convergence of beliefs and interpretive frameworks at community level. It ensured that the knowledge being embedded through personalised crossings was in keeping with the new way, and that it could be easily recognised within the community.

The micro level knowledge embedding mechanism was driven by ongoing boundary crossing between multiple engagement spaces. Boundary crossing also underpinned the macro-level embedding facilitating mechanism, although the boundaries crossed were at a higher structural level.

In the next chapter I outline the macro-level facilitating mechanism. 


\section{The macro-level mechanism: inter-CoP brokering}

\subsection{Introduction}

In this chapter I present my macro-level explanation for how online CoPs facilitated the embedding of professional knowledge at the level of a system of communities. Embedding of the new way at this level was driven and sustained by knowledgebrokering interactions between different levels, or tiers, of communities. The unofficial community of edubloggers, CoP E, acted as a critical bridging, or middle layer community between the ICT PD cluster CoPs and an international online CoP, linked to a larger global network. It can be seen as playing a key link in a knowledge value chain, brokering and converting knowledge as it moved both upstream and downstream. CoP E facilitated the embedding of knowledge by filtering and focusing inputs, reinforcing and contextualising knowledge, feeding it to local members and recycling key themes. It also provided a just-in-time support system, supporting the embedding of technology-based practices.

CoP E's knowledge-brokering function was carried out by individuals in two unofficial knowledge broker roles - the visible connector-leader and the invisible follower-feeder. Connector-leaders had a strong online influence in embedding knowledge, spanning the boundaries between online CoPs. Follower-feeders were local change agents who were largely invisible online, but had a strong offline influence. They followed connector-leaders and played a key role in brokering and embedding knowledge within school clusters by spanning the online-offline CoP boundary.

This chapter begins with an overview of the macro-level structure, explaining how CoP E functioned as a middle layer, knowledge-brokering structure within a tiered system of CoPs. I introduce the idea of a knowledge value chain and describe how the roles of the connector-leader and follower-feeder contributed to this. I outline a series of facilitating practices employed by connector-leaders and follower-feeders, and show how a combination of human and technological factors helped them to sustain the embedding of the new way. I then explain how the macro-level facilitating mechanisms helped drive the five embedding processes: focusing, persuading, aligning, adapting and owning. 


\subsection{The middle layer community}

Like platform $93 / 4$ in the novel Harry Potter (Rowling, 1997), the middle layer community (CoP E) was a non-official, in-between structure that was initially invisible to me. Prior to gathering data, I had envisaged the cluster-based CoPs (CoPs A, B, C and D) as sub-units of a national online CoP. This proved overly simplistic. At the national level, all clusters were formally connected via a website, a listserv, periodic online conferences and facilitated forums. However, in terms of active, day-to-day flow of professional knowledge, cluster CoPs proved to be more meaningfully connected in an indirect way, via their overlap with CoP E; part of a global online edublogging network.

CoP E was an open, non-official Web 2.0 community of movers and shakers (leaders, change agents and facilitators) from ICT PD clusters and affiliated NZ schools, based within a larger, global blogging community. Its members were united by a passion for, and belief in, the potential of ICT to transform teaching and learning, provided it was used in a student-centred manner and accompanied by critical reflection. These beliefs were strongly consistent with those underpinning the ICT PD programme, so becoming a member was an attractive proposition for those charged with spearheading change in the ICT PD clusters. The norms in this community of passion encouraged aligning with the emerging paradigm, ensuring a steady supply of persuasive arguments for the new way and practical solutions about how to implement it. The knowledge in CoP E was continually being refreshed, recycled and adapted on the community's inter-connected blogs. Community norms also ensured that its members received strong mutual support.

Joining the middle layer was a form of self-promotion that one person described as "moving into a bigger pond." Becoming an active member required one to cultivate a web-based presence, blogging persuasively about one's ideas and experiences and posting comments on the blogs of others. Although such groups are often referred to as networks, all eight active members of CoP E whom I interviewed stated that it was a community. (This supports Efimova and Hendrick's (2005) finding that online communities can exist in the dense areas of reciprocally linked blogs in networks.) One member explained that:

The community is very informal and fluid. I am of the opinion...that informal and fluid works much better. Formal and fixed communities such as exist for specific educational purposes tend not to have such a free flow of ideas.... People are scared of being wrong in those contexts and appearing stupid or ill informed. Whereas in this community...there is an easy nature about it. 
CoP E operated as a bridging, middle layer community, through which knowledge of the new way was brokered. (One person described it as "a middle layer of people, who are facilitating [KT], working between principals and schools'). It formed a horizontal middle layer by connecting up the cluster-based online CoPs to whom its members belonged. It also formed a vertical middle layer community in that it sat between the ICT PD communities and a global edublogging community. Analysis of blog linkages revealed a group of overseas bloggers with strong, multiple ties to CoP E (see Appendix F). This group included influential thought leaders, some of whom had visited as conference keynote speakers, as well as overseas teachers who were seen to be using ICT in effective, innovative ways.

The structure that emerged from my analysis was a system of overlapping online CoPs, similar to Castro's CoP Ecosystem of overlapping conversation spaces (2004, 2006), but with a tiered structure, reminiscent of an environmental ecosystem (see figure 36). There were three levels of CoPs and four tiers of participants: The first level of community was the cluster CoPs. The second level was the middle layer (CoP E) and the third layer was the global blogging community.

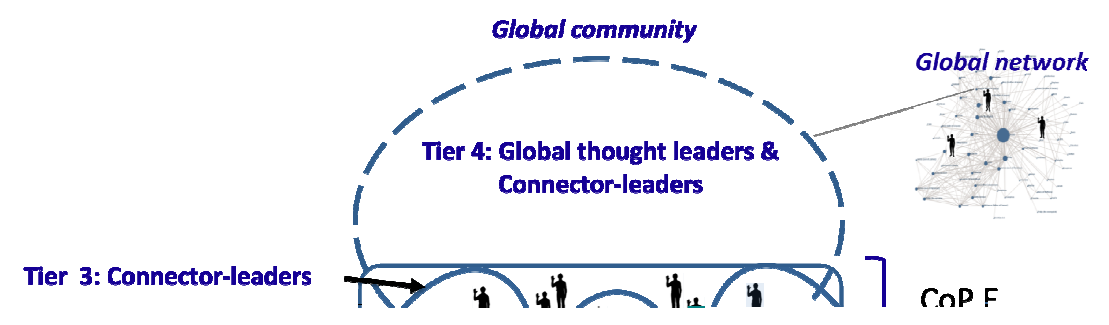


agents in cluster CoPs and invisible (non-blogging), peripheral members of CoP E. Tier 3 participants - connector-leaders - were change agents in cluster CoPs and also active members of CoP E and the global blogging community. Tier 4 participants were international thought leaders and connector-leaders who dominated the global community.

\begin{tabular}{|c|c|c|}
\hline Tier & Description & Quotes from interviews \\
\hline 4 & $\begin{array}{l}\text { Global thought } \\
\text { leaders and } \\
\text { connector-leaders }\end{array}$ & N/A: external to case \\
\hline 3 & $\begin{array}{l}\text { NZ connector- } \\
\text { leaders } \\
\text { (Visible, } \\
\text { active/blogging } \\
\text { members of CoP E) }\end{array}$ & $\begin{array}{l}\text { About tier 4: You've got really innovative and creative and motivational } \\
\text { people that are sitting up there at the top, and they're filtering their } \\
\text { ideas, and they're sparking conversations out... they're like the } \\
\text { conversation beginners. } \\
\text { About tier 4: They're out of my league; they're big names. }\end{array}$ \\
\hline 2 & $\begin{array}{l}\text { Follower-feeders } \\
\text { (Invisible, non- } \\
\text { blogging members of } \\
\text { CoP E) }\end{array}$ & $\begin{array}{l}\text { About tier 4: They're way up there, they know much more than I do. I } \\
\text { think if I say something, it might just sound really silly, so I don't say } \\
\text { anything.... I'm like a stalker } \\
\text { About tier 3: I'm a bit like a parasite. I take up her ideas, and I'm not } \\
\text { confident enough to give things back...But I am passing it on to people } \\
\text { below me. There are probably people feeding off me, who will never go } \\
\text { on-line, so I have to go out seeking more to give to them. } \\
\text { About tier 3: I look at people who I think are similar to me, or I find } \\
\text { myself having similar views to them... it gives a bit of weight to (my } \\
\text { ideas) } \\
\text { About tier 2: } \\
\text { I only communicated with one tier...making sure that I was on the right } \\
\text { page...via Skype and that sort of thing. } \\
\text { syndicate time. } \\
\text { We feed that [knowledge] back into our staff meeting time and our } \\
\text { syout tier }\end{array}$ \\
\hline 1 & Regular teachers & External to sample \\
\hline
\end{tabular}

Table 16: Quotes from interviews indicating hierarchical CoP structure 
A number of the participants whom I interviewed had an intuitive sense of this hierarchical system with its tiers of members (prior to me recognising it). Table 16 includes quotes that demonstrate this. One member of CoP E (a connector-leader) described the overall system as being like a spiral staircase, with the cluster-based change agents standing partway up. International thought leaders (the "conversation beginners") were at the top. Underneath them were other overseas bloggers who created opportunities for the NZ change agents to climb on board (global connectorleaders). Regular teachers were standing just below the change agents, following them. (Figure 36 simplifies the complexity of the overseas blogging community, which had sub-tiers, but was outside the case boundaries.)

\subsection{Knowledge brokers - driving embedding}

The tier 2 and 3 participants - visible connector-leaders and invisible follower-feeders in CoP E - played critical, complementary roles as knowledge brokers in this middle layer community, helping to drive the embedding of knowledge of the new way at a macro, or system of communities, level. I provide an overview of each of these roles below, with a discussion of their activities and how they facilitated embedding. (In Chapter 6, section 4.4.5, I outlined how these roles evolved during the decentralisation of ICT clusters' knowledge infrastructure in the Staying On Course stage of the KEC.)

\subsubsection{Visible connector-leaders}

Eight of those whom I interviewed were connector- leaders - core members of CoP E, whose opinions those in cluster-based CoPs followed. These people engaged in a high level of online activity, often being connected and available for over 12 hours a day. They used a wide range of online communication tools including blogs, RSS feeds, Twitter, IM tools, email and Elluminate. (Some also participated in private virtual communities such as Tapped In and Second Life.) When posting content on their blogs and on online forums, they selected themes that were consonant with CoP E's passion and beliefs surrounding the new way.

The blogs of connector-leaders employed a range of approaches. Some took a strongly pragmatic focus, emphasising ways of teaching with technology, addressing problems and telling success stories, while others took a more theoretical, analytical perspective, narrating on trends. Some steered a middle road, alternating between perspectives and/or regularly connecting up theory and practice. In addition, connector-leaders' blogs exhibited diverse personae. Some positioned themselves as experts, and others 
as ordinary, non-academic teachers with a passion for ICT. This corresponded with a division between connector-leaders with a theoretical orientation (thinkers) and those with a more practical bent (doers). The resulting thinker-doer duality in the CoP was well understood by participants. Some followers saw it as a polarity (two camps, one of which lacked self-criticality), but connector-leaders more often saw this as beneficial. It allowed them to build powerful reciprocal relationships (buddying), facilitating the matching up of theory and practice in a way that benefitted connector-leaders and their followers.

He's made me stop and think about, 'What are the reasons behind doing this? Why is this a good thing to do?, What's the motivation behind doing it? Is it really good teaching practice to be doing this? Is this really going to help the students learn?' (Connector-leader)

You can see the connection being made between the theoretical stuff to the practical [on the blogs].... I don't know if we'd do that very often otherwise. (Follower-feeder)

Over time, as the amount and range of their blog content increased, connector-leaders' blogs gained cumulative value. Interactions with thought leaders (who could be enticed to comment by tagging content with their names) increased connector-leaders'sense of recognition and status, while raising their game, encouraging them to operate at a higher level.

It grows and grows and grows, until you're putting a comment on really posh people's blogs. And they're putting comments on yours! (Connector-leader)

As unofficial knowledge brokers, connector-leaders bridged two key online boundaries: (a) the boundary between CoP E and the cluster-based online CoPs they worked with and (b) the boundary between CoP E and the international blogging community; a group of experts and educators who had similar beliefs about technology and a range of specialist expertise. (This is the distributed leadership system that schools began to depend on during the Staying on Course stage of the KEC). In relationship to these boundaries, connector-leaders were outward-facing in their identity as learners. They saw their online relationships as essential to their development, needing the support of like-minded others from the leading edge:

[When I had a problem] my blogging community was the one that made me feel better. It wasn't my colleagues in the classroom next door, because they couldn't offer me any advice...that wasn't how their classroom operated, and there really isn't anyone at school whose class operates like mine... There isn't anyone....as passionate about the technology in the classroom as I am. 
There was a strong spirit of mutual support amongst connector-leaders, enabled by $24 \times 7$ community mentality, message push services, and a spirit of reciprocity:

It doesn't matter what time it is; you can ask a question, you can ask for some feedback... say you've got a technical problem, you can put that out there... and your community will always come back.

As leaders, connector-leader played critical inward-facing roles. They facilitated the uptake and embedding of the way at the local cluster and school level, through a range of knowledge-brokering activities. I outline these below, after briefly describing the follower-feeder role.

\subsubsection{Invisible follower-feeders}

From the interview data, I identified a further group of eight less prominent knowledge brokers, whom I called follower-feeders. Follower-feeders were peripheral members of CoP E. They followed the blogs of one or more connector-leaders but did not post comments on these blogs, instead interacting with connector-leaders via email, IM and/or phone calls. Follower-feeders maintained a low online profile, being invisible outside of their closed, cluster-based online CoPs. They had neither the time nor the inclination to maintain a public blog, lacking the confidence to espouse their developing ideas in public. The focus of their roles as knowledge brokers was on bridging the online-offline CoP boundary. Follower-feeders were primarily inward-facing. They brought ideas that had been discussed online by connector-leaders into face-to-face contexts into the schools where they were change agents, helping their colleagues (who spent little, or no, time online) to access these new ideas and apply them in their teaching.

Over time, follower-feeders found themselves adopting similar beliefs to connectorleaders about the value of ICT-based learning:

I keep going back to see what else is there, and what they've found out." [I]: Do you feel that your thinking is becoming aligned with any particular people?

It's definitely developing, and along the lines of the 'e-learning is the beall and end-all' sort of thing... because it's the way of the future".

(Follower-feeder)

Even passion rubbed off onto the followers: 
I just like trying new things, and being up with it... because they seem to be so passionate about their learning. People who write these edublogs are really passionate. I guess some of it rubs off on me.

[I]: Interesting...

I'm not a leader, I'm just a follower.

I now consider a number of specific knowledge-brokering practices undertaken by connector-leaders and follower-feeders that contributed to the embedding of knowledge at the system of communities level.

\subsubsection{Knowledge-brokering practices of connector-leaders}

Analysis of data revealed a set of brokering practices performed by connector-leaders that contributed to the embedding of knowledge. These practices helped drive the five key knowledge embedding processes: focusing, persuading, aligning, adapting and owning.

\subsection{Filtering and focusing}

Connector-leaders selected specific foci to guide their online engagement, such as learning models, favourite theories, or ways of using a technology that supported the new way. They used these foci to screen and filter the large quantity of online content being produced daily, and to guide their own blog postings.

It's just getting an understanding of the way other people think, and seeing that there's bits and parts- maybe large parts, maybe small partsof what they're saying that fit with what I'm thinking, and what I believe. (Connector-leader)

Filtering also allowed them to check the alignment of their own thinking:

...what the on-line community manages to do is get the information you want a heck of a lot quicker, and from a variety of places, so you can validate it. And see if this stream of thinking is...in other people's places.

The job of filtering external blog content was in part devolved by connector-leaders to the upstream tier 4 participants whom they trusted:

There's about five people... I'll subscribe to the RSS feed in my Bloglines, and so I see everything that they stick on their Del.ici.ous ....'ve picked up some real gems...it's getting other people to do the work for you... I use other people as a filter. (Connector-leader) 
This first filtering step was facilitated by bloggers' use of a social bookmarking tool (Del.ici.ous) and RSS feeds. It was followed by a manual appraisal of quality described by one person as being like triage. Once connector-leaders had identified suitable content, they tagged selected content with relevant community themes (e.g. inquiry) for their followers:

I put it into my Del.icio.us, and I mark it for [Allan], or for [Susan], so they can link through to it that way.

Doing this created significant value for follower-feeders:

It's like going to the library, and rather than searching for your own good books, some nice librarian (comes up)... and says, "Here are fifteen books you might well be interested in"...these guys have filtered out a whole lot of good stuff, and so I can focus on reading and thinking about it.

Table 17 details the set of filtering and focusing practices and facilitating technologies used by connector-leaders: establishing foci; scanning, screening and filtering; following; filtering for quality; and sorting and classifying. These practices contributed to embedding by promoting and sustaining system level focusing on the new way.

\begin{tabular}{|l|l|l|}
\hline Practice & Technologies & Explanation \\
\hline $\begin{array}{l}\text { Establishing foci: Selecting } \\
\text { guiding/framing foci }\end{array}$ & RSS feeds & $\begin{array}{l}\text { Aids focusing; reduces noise of web } \\
\text { content }\end{array}$ \\
\hline $\begin{array}{l}\text { Scanning, screening and filtering: } \\
\text { and filter }\end{array}$ & RSS feeds & $\begin{array}{l}\text { Enhances focusing by aggregating } \\
\text { content on relevant themes }\end{array}$ \\
\hline $\begin{array}{l}\text { Following: Following respected, } \\
\text { influential people and/or colleagues }\end{array}$ & $\begin{array}{l}\text { Tagging/RSS feeds, } \\
\text { email, Twitter, Skype/ } \\
\text { iChat }\end{array}$ & $\begin{array}{l}\text { Facilitates aligning of ideas; } \\
\text { Topical shifts in themes keep ideas } \\
\text { fresh and create conversation hubs (re- } \\
\text { focusing) }\end{array}$ \\
\hline $\begin{array}{l}\text { Filtering for quality ("triage"): } \\
\text { Screening material for relevance and } \\
\text { quality }\end{array}$ & $\begin{array}{l}\text { Manual decision-making } \\
\text { supported by Skype / } \\
\text { iChat peer review }\end{array}$ & $\begin{array}{l}\text { Ensures attention is given to quality, } \\
\text { relevant material; condenses CoP } \\
\text { inputs (focusing) }\end{array}$ \\
\hline $\begin{array}{l}\text { Sorting and classifying: Sorting and } \\
\text { classifying content into familiar } \\
\text { categories (community taxonomy) }\end{array}$ & $\begin{array}{l}\text { Social bookmarking } \\
\text { (Del.icio.us ) }\end{array}$ & $\begin{array}{l}\text { Content is contextualised using } \\
\text { categories relevant to CoP, promoting } \\
\text { focusing and aligning (thematic } \\
\text { convergence) }\end{array}$ \\
\hline \begin{tabular}{l} 
Table 17: Filtering and focusing practices of comnector-leaders \\
\hline
\end{tabular}
\end{tabular}

Table 17: Filtering and focusing practices of connector-leaders 


\subsection{Reinforcing and contextualising}

Having identified relevant, quality material, connector-leaders reacted to it, recommending, reinforcing and recycling the incoming themes on their blogs. They increased the relevance and/or novelty of content to followers by extending themes, adding contextual commentary, juxtaposing and recombining ideas, and putting a new spin on a familiar theme. Packaging up familiar ideas in a new way helped to localise knowledge and generate novelty, while underlining and enriching key themes:

I've taken this bit from one person, this bit from someone else, and packaged it up differently. (Connector-leader)

New conversations are always happening, but then we're sometimes going back to the old conversation and putting a new spin on it. (Connector-leader)

Adding an original perspective to a thought leader's content created reciprocal benefits. It conferred authority by association on the citing author, and expanded the influence and blog ratings of the originator. The practice of tagging such postings with the cited authors' names (to enable discovery via RSS feeds) sometimes led to reciprocal commenting, setting up a virtuous cycle, and sustaining conversation on major themes. Stirring things up was another practice employed by connector-leaders helped promote embedding by challenging people, driving their deeper engagement with key ideas.

Connector-leaders also helped their followers by reflecting on the alignment between theory and practice.

[I]: I guess when you look at the blog, you don't know what you're going to find?

No, you don't. But... it is good, when you can see the connection being made between the theoretical stuff to the practical." (Follower-feeder)

Reinforcing content was a means of persuading others of the relevance of ideas, while adding contextual value promoted owning of knowledge at the local level. The set of Reinforcing and contextualising practices is detailed in table 18: Promoting, Extending, Stirring Up, Tagging, Commentating in a group, Remixing, and Echoing/resonating. 


\begin{tabular}{|c|c|c|}
\hline Practice & Technologies & Explanation \\
\hline $\begin{array}{l}\text { Promoting: Citing or } \\
\text { recommending a blog post or } \\
\text { presentation by another person }\end{array}$ & $\begin{array}{l}\text { Blogs, Online } \\
\text { Videos, Tagging, } \\
\text { RSS Feeds }\end{array}$ & $\begin{array}{l}\text { Amplifies significance of message; drives } \\
\text { followers to source (persuading others while } \\
\text { aligning with source) }\end{array}$ \\
\hline $\begin{array}{l}\text { Extending: Using someone else's } \\
\text { (referenced) blog post as a } \\
\text { springboard for one's own thoughts } \\
\text { (also described as piggybacking) }\end{array}$ & $\begin{array}{l}\text { Blogs, Online } \\
\text { Videos, Tagging, } \\
\text { RSS Feeds }\end{array}$ & $\begin{array}{l}\text { Adds local value and relevance by } \\
\text { contextualising content. Reinforces by } \\
\text { adding weight of local author, who gains } \\
\text { further credibility through association with } \\
\text { the cited material/author (persuading; } \\
\text { aligning) }\end{array}$ \\
\hline $\begin{array}{l}\text { Stirring up: As above, but } \\
\text { disagreeing with a referenced } \\
\text { source }\end{array}$ & $\begin{array}{l}\text { Blogs, Online } \\
\text { Videos, Tagging, } \\
\text { RSS Feeds }\end{array}$ & $\begin{array}{l}\text { As above, but may trigger deeper } \\
\text { engagement of readers with concepts as } \\
\text { they are challenged to take and justify a } \\
\text { stance (promotes stronger focusing) }\end{array}$ \\
\hline $\begin{array}{l}\text { Tagging: Tagging referenced } \\
\text { material with the originator's name }\end{array}$ & $\begin{array}{l}\text { Blogs, Tagging, RSS } \\
\text { feeds, e-mail }\end{array}$ & $\begin{array}{l}\text { Alerts the originator to a new, relevant } \\
\text { posting (focusing and aligning). This may } \\
\text { lead to the author responding, generating } \\
\text { further authority \& impact (persuading), and } \\
\text { deepening the conversation. }\end{array}$ \\
\hline $\begin{array}{l}\text { Commentating in a group: } \\
\text { Commentating on a blog or } \\
\text { conference keynote to } \\
\text { contextualise it, adding } \\
\text { local/personal opinion }\end{array}$ & Twitter, Skype/iChat & $\begin{array}{l}\text { Contextualises a real-time presentation, } \\
\text { promoting a shared interpretation (focusing, } \\
\text { aligning) }\end{array}$ \\
\hline $\begin{array}{l}\text { Remixing: Juxtaposing content } \\
\text { from different sources to make a } \\
\text { point; giving a new 'spin' }\end{array}$ & $\begin{array}{l}\text { Blogs, Tagging, RSS } \\
\text { feeds }\end{array}$ & $\begin{array}{l}\text { Novelty helps gain attention and can } \\
\text { generate new insights. May promote } \\
\text { persuading. }\end{array}$ \\
\hline $\begin{array}{l}\text { Echoing/resonating: Writing a } \\
\text { blog post that resonates with } \\
\text { previously introduced themes } \\
\text { (without referencing 'source') }\end{array}$ & $\begin{array}{l}\text { Blogs, Tagging, RSS } \\
\text { feeds }\end{array}$ & $\begin{array}{l}\text { Recycling familiar themes from a new angle } \\
\text { (aligning) reinforces concepts. Lack of } \\
\text { citation suggests owning of concepts. }\end{array}$ \\
\hline
\end{tabular}

Table 18: Reinforcing and contextualising practices of connector-leaders

\subsection{Feeding}

Connector-leaders were strongly aware of the needs of their local followers, going to considerable lengths to 'feed' them. This extended beyond posting blog content, to tagging and bookmarking material, and e-mailing followers the URL links to blog posts. This customised service helped teachers work out the next steps in their professional change journey. It resulted in follow-up discussions outside the visible community space, via e-mail, phone conversation and/or face-to-face, supporting the adapting process. 
Table 19 details the practices and technologies used by connector-leaders for Feeding and Helping others.

\subsection{Helping others}

Connector-leaders provided a voluntary just-in-time support service for each other and their followers, further facilitating the process of adapting. This service also helped to bind them to the CoPs to which they belonged and create a spirit of reciprocity. It was made possible by a culture of staying online for long periods. IM and Twitter were the tools of choice for seeking and providing assistance.

It doesn't matter what time it is, you can ask a question, you can ask for some feedback... say you've got a technical problem, you can put that out there into the Twitter world, and your community will always come back. (Connector-leader)

Competence with technology was essential for the successful delivery of the new way, so assisting each other with technical issues was important.

Connector-leaders supported cluster CoPs in implementing change through a practical matchmaking service, linking schools to external individuals with relevant specialist (teaching or technical) knowledge. They supported other connector-leaders who sought suitable quotes for their blog posts, feedback on emerging ideas, and input into communal resources, such as voice-threads. (Drawing together of complementary perspectives in communally developed resources served to mutually reinforce a core set of beliefs - persuading and aligning - and promote perseverance.) Real-time collaboration was also used to share successes (persuading) within the middle layer:

All but four in my class... are within a 6-month range of their age yippee! Must be the technology in my room tee-hee (Connector-leader, Twitter)

\section{Defending the community}

Sometimes Helping Others involved simultaneously Defending the Community. At times, change agents were challenged by individuals who disagreed with the new way - either by a reluctant from within their school or someone they had met at a conference or workshop. Connector-leaders reported on such episodes in their blogs, summarising the comments of the attacker. These challenges posed a threat to CoP E's core values and to those who had invested in the change journey. Such situations invoked a powerful community reaction, somewhat like the triggering of the human system's immune response by an antigen. A larger number of responses than usual 
were posted; combining combined the emotion and skills of persuading with the weight of aligning views.

My teaching role has never been, nor will it ever be, the entertainer and the kid's best friend. I use ICT and it's tools in my classroom because it's embedded in my teaching practise. It's not about using the flash-bang whizzy things that make learning "fun" - it's darn hard work... (Connector-leader, on another connector-leader's blog)

Table 19 details the practices and technologies used by connector-leaders for Feeding and Helping others.

\begin{tabular}{|c|c|c|}
\hline Practice & Technologies & Explanation \\
\hline \multicolumn{3}{|l|}{ Feeding } \\
\hline $\begin{array}{l}\text { Matching: Matching incoming online } \\
\text { (blog) content to known needs of clusters } \\
\text { and individuals }\end{array}$ & e-mail & $\begin{array}{l}\text { Ensures delivery of relevant content, } \\
\text { aiding ability of recipient to interpret, } \\
\text { embed and enact new knowledge }\end{array}$ \\
\hline $\begin{array}{l}\text { Passive feeding: Tagging content so it } \\
\text { can be accessed by others (see also } \\
\text { Sorting and Classifying above) }\end{array}$ & $\begin{array}{l}\text { Tagging, RSS } \\
\text { feeds }\end{array}$ & $\begin{array}{l}\text { Results in feeding of followers who use } \\
\text { RSS feeds and bookmarks; promotes } \\
\text { focusing }\end{array}$ \\
\hline $\begin{array}{l}\text { Active feeding: Alerting individuals who } \\
\text { have limited online time to specific } \\
\text { relevant blog/online content }\end{array}$ & $\begin{array}{l}\text { e-mail, } \\
\text { Skype/iChat, } \\
\text { Twitter }\end{array}$ & $\begin{array}{l}\text { Personalising content, combined with } \\
\text { individual attention, builds relevance } \\
\text { and owning; sustains focusing }\end{array}$ \\
\hline \multicolumn{3}{|l|}{ Helping others } \\
\hline $\begin{array}{l}\text { Being available: Community culture } \\
\text { involves long periods of being } \\
\text { continuously available online }\end{array}$ & $\begin{array}{l}\text { Twitter, } \\
\text { Skype/iChat }\end{array}$ & $\begin{array}{l}\text { Mutual facilitation of just-in-time } \\
\text { support service supports adapting and } \\
\text { promotes aligning }\end{array}$ \\
\hline $\begin{array}{l}\text { Sharing successes and problems: } \\
\text { Sharing and celebrating success }\end{array}$ & $\begin{array}{l}\text { Twitter, } \\
\text { Skype/iChat }\end{array}$ & $\begin{array}{l}\text { A form of persuading that sustains } \\
\text { beliefs and commitment }\end{array}$ \\
\hline $\begin{array}{l}\text { Testing and benchmarking: Testing out } \\
\text { ideas with colleagues, making } \\
\text { comparisons about ideas implemented in } \\
\text { different contexts }\end{array}$ & $\begin{array}{l}\text { Twitter, } \\
\text { Skype/iChat }\end{array}$ & $\begin{array}{l}\text { Practical support for embedding as } \\
\text { ideas and practices evolve; promotes } \\
\text { aligning }\end{array}$ \\
\hline $\begin{array}{l}\text { Brokering connections and solutions: } \\
\text { Brokering connections between local } \\
\text { community members and technology or } \\
\text { educational experts/practitioners }\end{array}$ & $\begin{array}{l}\text { Twitter, } \\
\text { Skype/iChat, e- } \\
\text { mail }\end{array}$ & $\begin{array}{l}\text { Practical support for followers as they } \\
\text { implement new processes and } \\
\text { technologies (adapting) }\end{array}$ \\
\hline $\begin{array}{l}\text { Defending the community: } \\
\text { Defending community members who are } \\
\text { under attack, using supportive } \\
\text { comments/arguments }\end{array}$ & $\begin{array}{l}\text { Blog, Twitter, } \\
\text { Skype/IM }\end{array}$ & $\begin{array}{l}\text { Reinforces community beliefs and } \\
\text { asserts aligning. Bolsters individual } \\
\text { morale by defending against non- } \\
\text { aligned views }\end{array}$ \\
\hline
\end{tabular}

Table 19: Feeding and Helping others: practices of connector-leaders 
The strength of the community's concern with threats was evident in an example when a change agent reported overhearing a muttered comment, 'When in ten years time it doesn't work we will all just go back to how it was anyway'." This drew a strong defensive response from seven CoP E members. In another case, a blog post reporting an overt challenge drew eight defensive responses with a combined total of over 2,500 words. Defending against challenges reasserted the CoP's beliefs and values (persuading). At a practical level, it supported the person who had been attacked.

It's how you deal with adversity that is more telling than experiencing success.... taking turns at being the damp sponge is essential - how else would we recognise and value our moments as the bomb? And if it always goes to plan with a hiss and a roar its much harder to figure out how to improve - this way whatever happens the next time you share your thinking with others you will feel better about the outcome (Defensive response, connector-leader's blog).

Change agents were critical to embedding within schools, so were vulnerable to attack, and needed one another's support. Despite this, individuals in CoP E recognised that there was value in being provoked:

Reading this post I was provoked - I believe this is a good thing, now there is a flurry of thoughts whirling round in my mind that I feel compelled to share... (Connector-leader, blog)

Being challenged was also valued as a way of militating against over-alignment and safeguarding against what one person called "blog evangelism". It kept connectorleaders' thinking fresh and encouraged critical reflection on, and fresh justification of, the new way.

[That person was] challenging us...to justify why we think what we're thinking. That's good for any community...that's the only way that we move forward, and solidify our position... Too often a community is 'closed'...We share similar pedagogical ideas and beliefs, which encourages one another... but we are also in danger of becoming a community of self-congratulatory nodding dogs. (Connector-leader, interview)

\subsubsection{Knowledge-brokering practices of follower-feeders}

Because follower-feeders spent a restricted amount of time online, they selected only a few connector-leaders, whose blogs they followed. They also actively communicated with these people, using email and instant messaging (IM) tools as invisible backchannels to follow-up their blog posts and seek their opinions and advice. 
I can talk to her via Skype, and she's very approachable, and can teach us about just (anything) ...you know, give her a call. Like interviewing with the kids... using Bubbleshare and all sorts of things. I brought [her ideas] back to our group, and said, "Have you tried this?"'

[I] So you've read her blog and then you go back and Skype her?

Yes. (Follower-feeder, CoP B)

Once follower-feeders had become regular behind-the-scenes blog correspondents, connector-leaders kept them up to date with their blogs by e-mailing (feeding) them

URLs. (Unlike connector-leaders, follower-feeders did not use RSS feeds to keep track of the blogosphere, relying instead on personal recommendations and visits to bookmarked URLs.)

It was notable that in the interviews, a number of follower-feeders used metaphors of feeding on the ideas articulated by connector-leaders:

She was like someone in the food chain I could feed off. (Followerfeeder, CoP B)

I don't get...to go to conferences...so (I) pop in there and scoff their knowledge and away again... (Follower-feeder, CoP B)

Follower-feeders saw themselves as feeding on the ideas of those whom they perceived as being above them, and then feeding this knowledge on to those below them, as they adapted and recycled themes in new contexts:

I rely on Rebecca. She spends hours and hours and hours looking at blogs on the net. She finds anything that's worthwhile, and she'll alert you to it... I'm a bit like a parasite. I take up her ideas, and I'm not confident enough to give things back. But I am passing it on to people below me. There are...people feeding off me, who will never go on-line so I have to go out seeking more to give to them. (Follower-feeder, CoP B)

Their passing on of ideas fostered localisation of knowledge, building intra-school ownership. The feeding language was strongly suggestive of a multi-layered food chain in which food (in this case, knowledge) was being reused and digested by successive levels of consumers. This recycling system, brokered by both the connector-leaders and follower-feeders, facilitated knowledge use in the local context by ensuring its relevance to consumers. Knowledge gained value as it was varied, amplified, and enriched by knowledge brokers.

At the macro level, recycling of dominant themes resulted in the aligning of thinking through the repetition and saturation of powerful themes. A lead teacher in CoP B 
spoke of a thread of ideas that kept coming through in their interactions with lead teachers from other schools. One follower-feeder spoke of having experienced this in relationship to inquiry learning:

It seems to me that there were a lot of things coming at once.... there were a lot of things out there ... about inquiry learning, and those sort of philosophical shifts. And you sort of just read stuff. But it was major, and through communication with the other schools that were involved in it, we started to change the way we'd done things here, and basically threw out our curriculum plan... and started again. (FF, CoP A)

The follower-feeders played a complementary role to connector-leaders. They helped to extend the reach of connector-leaders' knowledge by transferring and embedding knowledge in the workplace community. Their role brokering knowledge across the online-offline boundary of CoPs was particularly invaluable because there were multiple barriers to online community engagement in the day-to-day working environment of the regular teacher: Teachers often saw it as inappropriate to engage in online peer-to-peer dialogue during teaching hours. The vast majority of their online time was directed towards the immediate benefit of students, while non-contact time hours and evenings were spent on individual lesson planning, using the Internet as a resource. (In the case of follower-feeders this had changed: IM was used to engage with colleagues in the course of lesson planning.)

Regular teachers relied on follower-feeders to interpret, adapt, and pass on the ideas and advice of the connector-leaders and to find solutions when they got stuck. One follower-feeder described how they screened connector-leaders'ideas with the needs of the regular teacher in mind:

I check things out prior to telling staff. I guess I make decisions about what will work, and what not to tell them... (Follower-feeder, CoP B)

The impact of follower-feeders was invisible and impossible to measure, but it is hinted at in the following comment in a forum by a connector-leader who was a facilitator:

I never worry too much when I don't see the forums take off with every teacher commenting, sharing ideas and asking questions... It is more likely that people will have their discussions a week or two later, when they are starting to struggle a little with the new things they are doing...Even then the conversations may still be face-to-face with their cluster facilitator, or behind the scenes with an email to one of us, or a phone conversation at the moment that they are stuck on something. But the networks have grown. The contacts have been identified. 


\subsection{Sustaining embedding: the knowledge value chain}

The knowledge-brokering practices outlined above suggest a one-way, top-down flow of knowledge, but the reality was more complex. Knowledge brokering by the middle layer community was a reciprocal, two-way process. It occurred in both top-down and bottom-up directions, resulting in a cyclic value chain of knowledge. In order to explain this I use an example from CoP B.

Figure 37 shows how online interactions between the four tiers of participants (all given pseudonyms) facilitated KT in a subset of CoP B (the shaded area), helping foster new ideas and practices in three schools. (Lines with arrows indicate blog followers. Dotted lines show IM communication.)

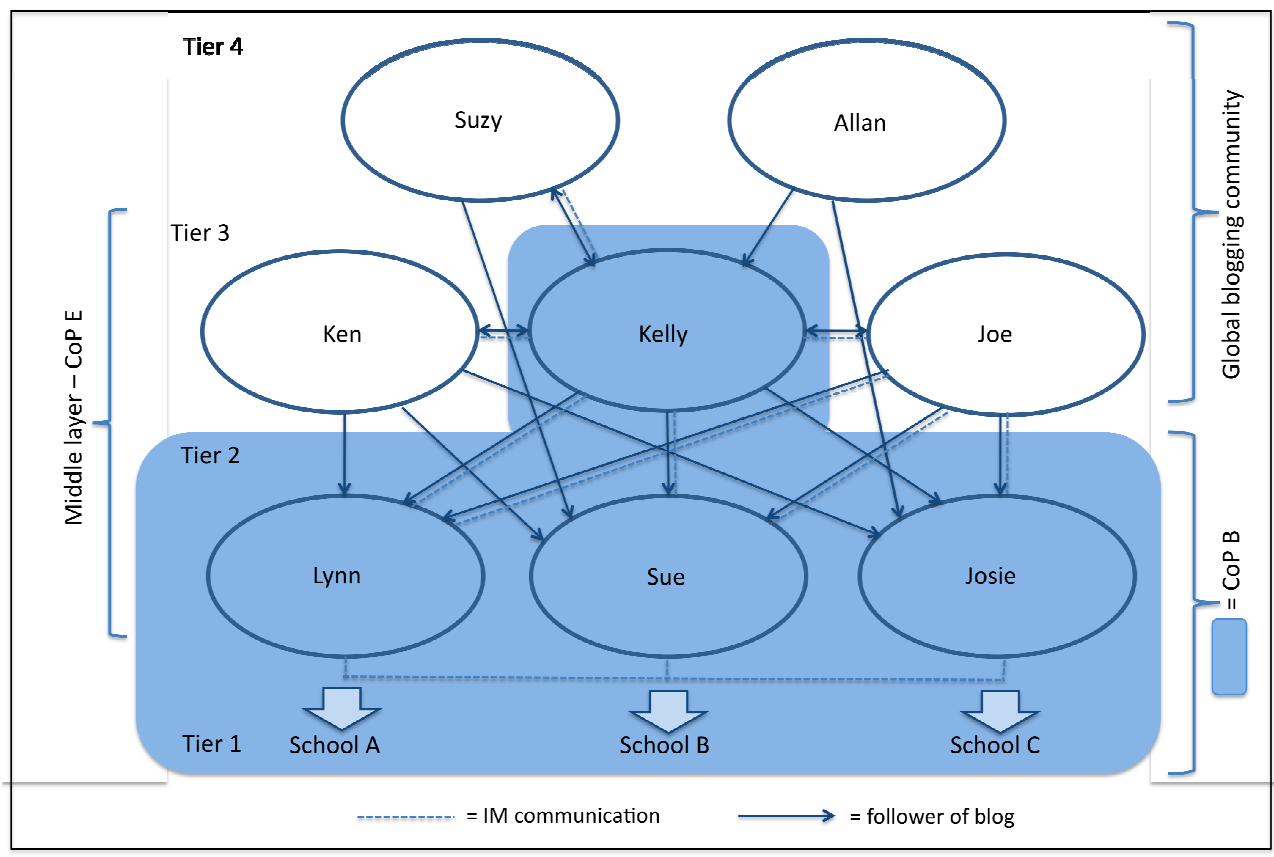

Figure 37: Interactions between tiers of system participants in a subset of CoP B

Kelly, a key member of CoP B, was a connector-leader with strong links to a number of tier 4 participants. (Two of them, Suzy and Allan, are shown in the diagram.) Kelly played a key role in synthesising, interpreting and brokering knowledge from tier 3 and 4 bloggers, via her blog and IM (as well as via email, Twitter and other tools). Three follower-feeders, Lynn, Sue and Josie regularly followed her blog. They also followed the blogs of Kelly's more theoretically orientated peers, Ken and Joe. Sue sometimes followed the blog of one of Kelly's tier 4 contacts, Suzy. Josie did likewise with Allan's blog. Lynn, Sue and Josie regularly engaged in IM dialogue with each other, Kelly and (less often) with another tier 3 participant, Joe. They relied on Kelly to acquire knowledge from other tier 3 and tier 4 participants. 
Lynn discovered that by following Kelly's blog she reduced the time needed for knowledge seeking, allowing her to focus her energies on meeting the needs of her followers. She passed on the knowledge acquired from Kelly (and her blog) to these followers, regular teachers in School A, via face-to-face means. Sue performed the same service for School B, and Josie for School C.

I'm picking up ideas. If they work in my room, I share them with other people. (Lynn)

The evolution of teaching practices in CoP B reflected the knowledge flows shown in figure 37: Lynn, Sue and Josie followed Kelly's lead, taking up student blogging. They followed Kelly's broad approach and technical advice, adapting the practice to work in their own contexts. Others in their schools had begun to do likewise. Because Lynn, Sue and Josie followed (some of) the same bloggers as Kelly, they were exposed to the same set of theoretical ideas. When they spoke via IM and face-to-face they were on the same wavelength and had no difficulty aligning their understandings.

Although Lynn initially told me that that she gave nothing back to Kelly, this was not the case in reality: Lynn had implemented a non ICT-based practice (picked up from another school), and Kelly had adapted it, creating an online version which she featured on her blog and shared with tier 4 colleagues. Lynn's inward-facing role had provided her with access to a novel practice that was of value to Kelly in her outwardfacing role. By adapting this practice so that it better aligned with the new way Kelly could enhance her street cred, creating new opportunities for others to engage with her blog, which in turn would build further value for her followers.

The flows of knowledge therefore resulted in a two-way knowledge value chain: Tier 4 participants were the system-level leaders in terms of generating knowledge about the theory of the new way (why knowledge). They did this by generating powerful new themes and metaphors and commentating on events. As these why ideas moved downstream they were fed on and adapted, adding weight to the ideas of those in tiers 2 and 3 as they pushed for change in their local clusters level. On the other hand, those participants who were closest to the coalface (tier 1, 2 and 3 teachers who worked in classrooms) were at the leading edge when it came to generating knowledge about how to successfully apply the new way in practice. One CL explained why it was essential for such knowledge to move back upstream to those in tier 4:

[I]: Thinking about the staircase, what do you see as the benefit [of your blog content] for those people who are at the higher level? 
It makes them reflect on what is actually working. Because there must be a huge amount of pressure... When you share something [on your blog] you can be really enthusiastic about it, but if it's actually not worked that well...You would have to be really careful if you were way up there on the staircase, because others will follow in your footsteps.

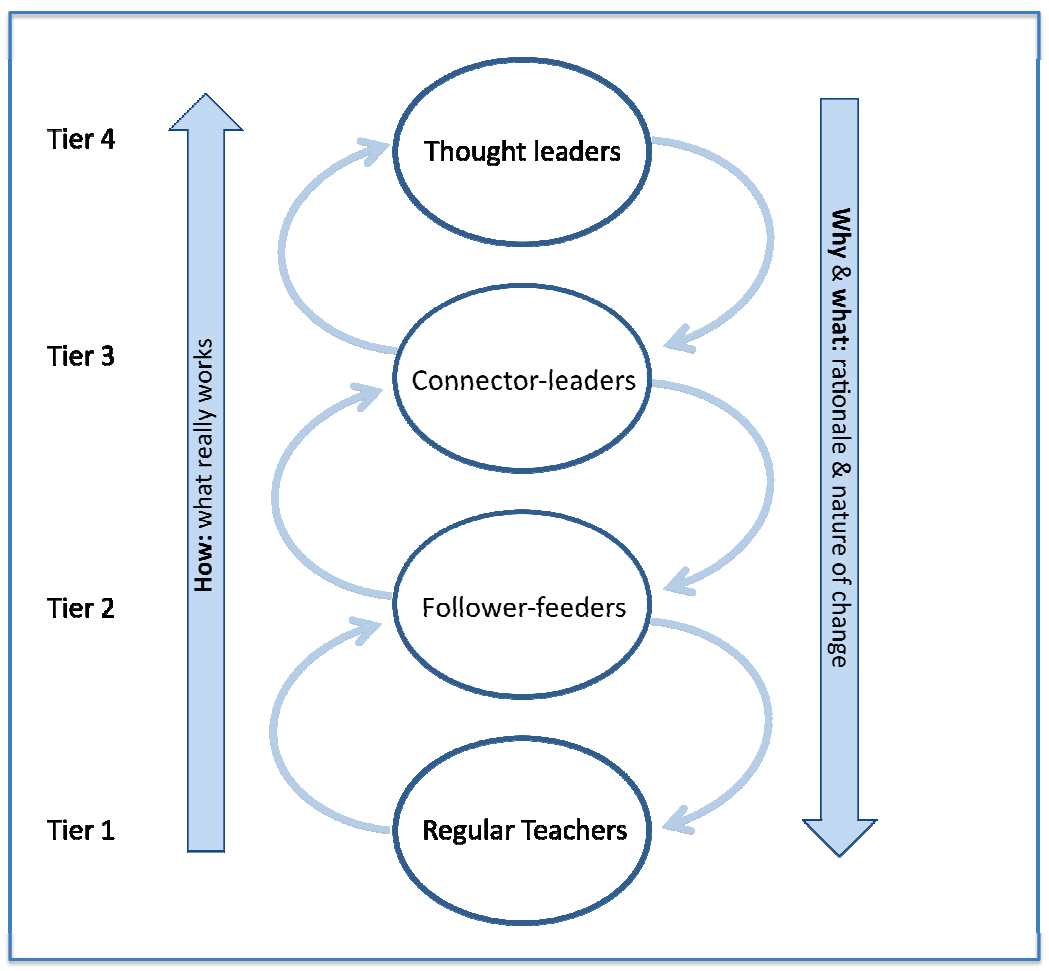

Figure 38: Knowledge value chain: reciprocal flows that drove and sustained embedding

In other words, tier 4 participants needed to ensure they were in touch with what worked in practice in order to minimise risks to their credibility. The tier 2 and 3 participants - follower-feeders and connector-leaders - therefore played a reciprocal role as knowledge brokers, facilitating both upstream and downstream knowledge flows. The nature of their role in the knowledge value chain is illustrated in figure 38. This can be seen as a means of sustaining the embedding of the new way at system level by ensuring a balance of theoretical and practical knowledge, while maintaining currency and novelty through continual recontextualisation. 


\subsection{The macro-level mechanism and the five embedding processes}

The macro-level facilitating mechanism of inter-community knowledge brokering promoted all five of the knowledge embedding processes: persuading, focusing aligning, adapting and owning. I have already provided a number of examples of how this occurred at the level of facilitating practices. Table 20 summarises the key macrolevel drivers of the five processes.

\begin{tabular}{|c|c|}
\hline Process & Ways in which promoted by inter-community brokering \\
\hline Persuading & $\begin{array}{l}\text { The middle layer community (CoP E) was a community of passion. It filtered and } \\
\text { brokered knowledge through a 'passion' lens. } \\
\text { The filtering and focusing of connector-leaders ensured a ready supply of } \\
\text { persuasive material that was enhanced by reinforcing and contextualising. } \\
\text { Connector-leaders fed persuasive arguments to each other and to follower- } \\
\text { feeders, who recycled them in offline settings. }\end{array}$ \\
\hline Focusing & $\begin{array}{l}\text { CoP E drove focusing on content that was relevant to both the new way and local } \\
\text { CoPs. This was driven by the filtering and focusing practices of connector- } \\
\text { leaders and the feeding practices of connector-leaders and follower-feeders. } \\
\text { Facilitating technologies (RSS feeds, social bookmarking tools) enabled these } \\
\text { practices. }\end{array}$ \\
\hline Aligning & $\begin{array}{l}\text { Intercommunity brokering by CoP E drove system-level aligning by sustaining } \\
\text { 'common threads' of themes across CoPs. CLs amplified the impact of influential } \\
\text { edubloggers by reinforcing practices (e.g.,echoing, remixing), while } \\
\text { contextualising increased local relevance. } \\
\text { Helping Others (e.g., testing and benchmarking, brokering connection and } \\
\text { defending the community) sustained aligning. Connector-leaders aligned in } \\
\text { defensive clusters when under attack. } \\
\text { Facilitating technologies make it easy to identify and follow aligned content and } \\
\text { enabled the tagging of content with community themes (social bookmarking, RSS } \\
\text { feeds). }\end{array}$ \\
\hline Adapting & $\begin{array}{l}\text { CoP E supported system-level adapting by creating rapid connections between } \\
\text { people and the ideas, people and technologies that could help them. Helping } \\
\text { Others and the community norm of being always online were the key drivers. } \\
\text { Twitter (and IM tools) facilitated the making of real-time connections and a just- } \\
\text { in-time support service that operated across the CoPs and network.. }\end{array}$ \\
\hline Owning & $\begin{array}{l}\text { In the course of 'downstream' brokering, CoP E fostered the owning of } \\
\text { knowledge by increasing local relevance (through the Reinforcing and } \\
\text { Contextualising practices of connector-leaders) The strong owning of ideas } \\
\text { exhibited by connector-leaders in their blogposts helped influence followers. } \\
\text { Connector-leaders solicited input into communal resources, such as voice- } \\
\text { threads. Drawing together of complementary perspectives in this way enriched } \\
\text { core beliefs while promoting owning amongst distributed members. }\end{array}$ \\
\hline
\end{tabular}

Table 20: The macro-level mechanism and the five knowledge embedding processes 


\subsection{Chapter summary}

In this chapter, I have outlined how the embedding of the new way was driven at the macro, or system of communities, level by the knowledge-brokering function of a middle layer community of bloggers and their followers; CoP E. During the Staying on Course stage of the KEC, as schools' and teachers' needs became more specialised, a greater range of knowledge was needed to support them in their change journey. As CoP E evolved to become a community of passionate knowledge intermediaries, it proved ideal for supporting these needs. It was characterised by a devotion to the new way, overlapping membership with the ICT PD programme, and international connections.

CoP E functioned as the hub in a knowledge value chain. It filtered, brokered, reinforced and recycled knowledge of the new way amongst different levels of CoPs, in ways that promoted the five knowledge embedding processes. This activity was based around the brokering practices of visible connector-leaders and invisible followerfeeders. My discovery of these two complementary types of knowledge brokers, spanning two different types of boundaries - online CoP boundaries, and the onlineoffline boundary - was significant. It illustrates the fact that multiple boundaries exist in today's complex CoP environment, and underlines the potential specialisation involved in knowledge broker roles.

In particular, my discovery of the critical role of follower-feeders challenges assumptions about the lurker, helping address a lack of understanding about this role (Nonnecke, et al., 2006; Nonnecke \& Preece, 1999; Nonnecke et al., 2004; Rafaeli, et al., 2004; Soroka \& Rafaeli, 2006). Assumptions that lurkers provide no benefit to their wider community appear to arise from a lack of recognition of the importance (perhaps even the existence) of the online-offline boundary, and the need to transfer knowledge across it. In CoP E, the activity of follower-feeders was invisible, owing to their backchannel interactions with connector-leaders. Furthermore, their visible activity in local online communities did not reflect their brokerage role with respect to connectorleaders. Although they were keen to avoid leaving a digital footprint, they created real CoP impacts.

In keeping with Castro's $(2004,2006)$ ecosystem view of CoPs, two ecological themes arise from this macro-level perspective of the case. The first theme is that of a knowledge food chain, or value chain, where valuable knowledge passed through an 
ecosystem, being reconstituted and re-used by different levels of consumers. The second ecological theme is that of interdependence, or symbiosis. Connector-leaders and follower-feeders performed symbiotic, mutually dependent roles as knowledge brokers. Connector-leaders gained a sense of value and recognition through their interactions with follower-feeders, and secured practical examples of knowledge application that would be valued and enhance their credibility in the eyes of international experts. Follower-feeders, in turn, relied on the connector-leaders as gatekeepers and purveyors of quality, for keeping their ideas current and relevant, for helping them solve problems, and for connecting them to others who were working in similar areas. The embedding of knowledge at macro level was strongly facilitated by their symbiotic relationship.

The next chapter is the final one in this thesis. In it, I summarise the three different levels of findings in relationship to my research question, and consider their implications for the IS field and for practice. I also highlight the limitations of this research, relate my meso-, micro- and macro-level theories to the existing theoretical literature, and suggest directions for future research. 


\section{Conclusion}

\subsection{Introduction}

This research project has investigated the process of professional change from a knowledge management (KM) perspective. Its aim was to elucidate how online CoPs can facilitate the embedding of new professional knowledge in the context of transformative professional change. My project used a case research design (Yin, 2003) based on a single, unique case; the NZ Ministry of Education's ICT Professional Development programme for schools. The case had five sub-units: four official online CoPs (the online dimension of blended school cluster communities) and one unofficial, virtual CoP with a distributed membership. The study focused on identifying and explaining the mechanisms, roles, technologies and other factors that facilitated the embedding of knowledge of the new way, an emerging, student-centred, ICT-enabled teaching paradigm.

This was an interpretivist study in which I used qualitative research methods with the aim of producing rich insights into a phenomenon about which there was little previous knowledge. Such an approach is considered appropriate when one is conducting research into an emergent area of understanding. However, it inevitably results in what some people would consider a trade-off: The findings are typically rich and deep, but they are also highly contextual. This means they typically cannot be readily transferred to other contexts. Additional research, building on case-bound results, may be necessary in order to create the kind of generic theory that can be applied in a broader range of settings.

In this final chapter of my thesis, I consider the issues outlined above. Firstly, I review the nature of my research results - a multi-level theory for explaining how online CoPs can facilitate the embedding of knowledge. I then outline the key limitations of my study. Following this, I review my research findings, summarising the answers that they have provided to my research questions, before moving on to discuss the implications of this research. I consider the potential significance of my findings in relationship to the IS field, the research context and the wider world of practice, suggesting possible directions for future research. 


\subsection{The nature of my research results}

My research has produced a multi-level (three-tier) theory for explaining how online CoPs can facilitate the embedding of knowledge. These results, outlined in Chapters 68 , represent my subjective view, as an interpretivist researcher, of the factors that were most significant for explaining the phenomenon I investigated. The interpretive paradigm views reality as an entirely subjective construct in which "facts and values are inextricably linked" (Charmaz, 2006, p.127), valuing the richness of insights over so-called universal truths. My results may differ from how others would interpret the data, but through their satisfying of the criteria for methodological plausibility, credibility and consistency (Gregor, 2006, p.625), as outlined in Chapter 3, they can be seen as having trustworthiness and as being worth paying attention to (Lincoln \& Guba, 1985, p.290). In order for interpretive studies to be of value, they should also lead to conclusions that have some degree of generality or transferability (Gregor, 2006; Klein \& Myers, 1999). I discuss how aspects of my theory meet this criterion in the implications section of this chapter.

Interpretivist researchers are exhorted to facilitate a depth of imaginative understanding by discovering and communicating rich contextual insights, patterns and connections (Charmaz, 2006, p.126). My explanatory theory - comprising three complementary mechanisms, at the micro, meso and macro levels, driving five key knowledge embedding processes - aims to generate this kind of understanding about how online CoPs facilitated the embedding of professional knowledge in my research context. Theory that has been generated through interpretive methods is not renowned for simplicity. This is in part because interpretivism considers the simultaneous existence of different explanations for events as a source of value. My development of multi-level theory is synergistic with this view.

In the field of Organisational Science, multi-level theorists have argued that different perspectives are equally valid for understanding organisational change, and also that any given theoretical perspective can provide only a partial explanation for a complex phenomenon (Edwards, 2005, p.269). The act of choosing a level of analysis as a focal lens is seen as placing constraints on the nature of the theory that will arise from research into a given phenomenon (Drazin et al., 1999). Because of such constraints, Edwards (ibid), Klein et al. (1999), Van de Ven and Poole (2000) and others have suggested that there is value in exploring the interplay between different perspectives. This is seen as providing possibilities for the development of novel and useful insights 
and theories, which may have broader explanatory power (Van den Ven and Poole, ibid). Similar issues exist in the IS field, where researchers have highlighted a limiting bias in the body of research towards a meso, or organisational level of analysis (Crowston \& Myers, 2004).

I did not set out to produce a multi-level theory - it emerged from the complexity of my data and my desire for a holistic answer to my research question. However, it can be seen as an appropriate theoretical outcome of this study for two reasons: Firstly, my study was based in an area of systemic concern: the NZ government's aim to improve effective teaching at a national level. Ecological theorists in the social sciences have highlighted the need to acknowledge the complexity of change initiatives targeted at a system level by utilising different levers at different levels of intervention (McLeroy et al., 1988). My theory is not ecological in the tradition of Bronfenbrenner (1979), but it is layered in a way that recognises the complementary nature of the knowledge embedding processes that were simultaneously occurring at different levels. A singlelevel theory - even a macro-level theory - would not have produced sufficiently granular explanatory theory to describe the complexity of the knowledge embedding mechanisms that were occurring at each level.

Secondly, multi-level theory is appropriate because of the complex way in which online CoPs manifested themselves in my study context. Online CoPs proved to be significantly more complex that I had anticipated. They operated beyond the boundaries of the school clusters, eroding traditional organisational constraints and opening schools to new influences. I found that a meso-level analysis on its own was insufficient to explain the way in which online CoPs were sustaining and driving change in my research case. In particular, schools were dependent on the micro- and macrolevel knowledge embedding mechanisms for helping drive the later stages of the knowledge embedding cycle, and for sustaining professional change.

In summary, I view my multi-level theory as a suitable way of acknowledging the complexity of the emerging online community environment - or online CoP ecosystem (Castro, 2004, 2006) - in which not only teachers, but many of today's professionals are operating. Taking a multi-level perspective in my analysis of data allowed me to take into account the genuine complexity of the online community environment and knowledge embedding processes operating in the research setting, ensuring the relevance of my findings. It has also provided me with a coherent and structured way of interpreting this complexity, contributing to the rigour of my theory. 
My research project has produced explanatory theory; a type of theory that Gregor (2006) sees as providing a dual source of value to the IS field. The first source of value lies in the research results themselves - in the degree to which they are novel (new and interesting, ibid, p.625) and/or significant (explaining "something that was poorly understood beforehand', ibid). The ways in which my theory can be seen as novel and significant are outlined later in this chapter. The second source of value lies in the potential of explanatory theory to be a vital ingredient for future, value-adding kinds of IS research: While explanatory theory does not aim to be predictive, it can contribute to the creation of theory for explaining and predicting and theory for design and action (ibid). I consider the potential of my research in this respect towards the end of this chapter.

\subsection{Summary of the research findings}

\subsubsection{Deriving order from complexity}

In this section, I summarise the way in which my multi-level theory of knowledge embedding in online CoPs has answered my research questions. I begin by restating the research questions, then recap on why I took a multi-level approach in addressing them. Finally, I summarise the answers each level of theory has provided.

My research project was guided by the question:

- How do online communities of practice (CoPs) facilitate the transfer and embedding of professional knowledge?

and the subsidiary questions:

- What is the nature of the knowledge embedding process in online CoPs?

- What technologies, roles, and other factors help online CoPs to embed knowledge?

As outlined previously, my focus was on articulating the process and facilitators of deep knowledge transfer, or knowledge embedding, in the online CoP context. My case research design was initially based on a nested approach, comparing four CoPs within a unique case; the ICT PD programme. I had envisaged that these CoPs would fit with traditional conceptions of the online CoP; facilitated groups of professionals who interact and share knowledge in a common online space. However, I discovered a very different reality: The online CoPs to which my research participants belonged were complex. They had visible and invisible, and official and unofficial 
dimensions. Much of members' interaction was not facilitated. Most importantly, these CoPs did not, on their own, account for how knowledge was being embedded in the research setting.

My discovery of CoP E, a highly active, fully virtual CoP, centred around blogs - which I initially saw as compensating for the fact that one of the other CoPs was inactive was significant. It was clear that this $\mathrm{CoP}$ (CoP E) was critical to understanding how the new professional paradigm was being embedded. It had overlapping membership with the other CoPs - indicative of likely knowledge transfer - yet it did not match my initial conception of an online CoP. When I revisited the literature, I found there were precedents for viewing it as an online CoP (Efimova \& Hendrik, 2005; Hodkinson, 2006). I amended my case design to include this community. This was also a decision to enlarge my perspective, viewing the CoPs in my study as a system of communities.

I encountered a similar level of complexity when it came to identifying the mechanisms that were facilitating embedding. Some of my data accounted for the way in which the new paradigm was being embedded at school and cluster level, while other data was illustrative of embedding at a higher, system of communities level. As I recognised the complexity of the online CoPs, I also began to gain an understanding of how knowledge embedding was operating at the level of the individual. To add to the above complexity, some of my data suggested that there were distinct stages of embedding, while other data was indicative of an ongoing, continual, process. Gradually, my data began to coalesce into three distinct themes.

I saw two options for moving forward. The first was to focus solely on articulating an organisation-centric (meso-level) explanation for how online CoPs were promoting knowledge embedding. This would mean ignoring my other findings in order to gain a depth of single focus. The second option was to develop a structure that would impose meaningful order on the complexity. This would entail sacrificing a single storyline in favour of a richer, more holistic and more original set of findings. I saw the latter option as a way of making a more valuable contribution to IS research, and as it was in keeping with the interpretivist tradition, I opted for this option, using three perspectives to structure my theory.

I have accounted for the complexity in the research setting by producing explanatory theory from three perspectives or levels of analysis - meso, macro and micro. The result is three complementary views; three sets of answers that are equally and 
simultaneously true; for explaining how online CoPs facilitated the embedding of paradigmatic professional knowledge in the research case. I now briefly review the three theoretical perspectives and discuss the nature of their relationship, before summarising how each one addresses the research question.

\subsubsection{Three complementary perspectives: meso, micro and macro}

My meso-level findings (Chapter 6 ) focus on how the knowledge embedding process occured at the level of the organisation and its associated $\operatorname{CoP}(\mathrm{s})$. The results at this level emphasise the importance of change in the organisational state over time, focusing on the role of CoPs in facilitating the progression from state to state as knowledge was gradually embedded. My theoretical findings at this level provide a structure (the KEC) for explaining knowledge embedding as a staged organisational change process and for considering the role of online CoPs at each stage. Online CoPs played an important, but changing, role in facilitating knowledge embedding as schools faced different issues over time. Table 21 provides a summary of how my meso-level theory addresses the research questions.

My findings at the macro and micro levels of analysis provide complementary perspectives. At both these levels, I have viewed the embedding of paradigmatic professional knowledge as a continual process, identifying ongoing mechanisms that explain how online CoPs drive knowledge embedding. My theoretical explanations at these levels are strongly interpretative. Both are based on the concept of boundaryspanning activity. 


\begin{tabular}{|c|c|}
\hline \multicolumn{2}{|c|}{ Meso-level theory: Overview } \\
\hline \multicolumn{2}{|c|}{$\begin{array}{l}\text { Online CoPs played an important, changing role in facilitating the embedding of knowledge, as } \\
\text { schools faced different issues over time. Schools embedded the new way through a six-stage } \\
\text { knowledge embedding cycle based around school-specific foci, or approaches. Online CoPs } \\
\text { facilitated a unique set of embedding sub-processes at each stage, contributing to the key } \\
\text { knowledge embedding processes; focusing, persuading, aligning, adapting and owning. }\end{array}$} \\
\hline \multicolumn{2}{|c|}{$\begin{array}{l}\text { Question 1: How do online CoPs facilitate the transfer and embedding of professional } \\
\text { knowledge? }\end{array}$} \\
\hline & $\begin{array}{l}\text { By facilitating a set of embedding activities at each stage of the } \\
\text { knowledge embedding cycle. These drove the five fundamental knowledge } \\
\text { embedding processes; focusing, persuading, aligning, adapting and owning. }\end{array}$ \\
\hline & $\begin{array}{l}\text { By establishing a culture of professional dialogue that infiltrated into the workplace, } \\
\text { transforming norms to create an environment conducive for the embedding of the } \\
\text { new paradigm. Workplace conversations reinforced and enriched further online } \\
\text { communications, creating a virtuous online-offline cycle. }\end{array}$ \\
\hline & $\begin{array}{l}\text { By challenging the status quo though the seeding and cultivating of powerful } \\
\text { reframing themes, leading to their appropriation by the CoP }\end{array}$ \\
\hline & By facilitating the iterative matching up of theory and practice \\
\hline & $\begin{array}{l}\text { By providing a shared repository for (a) school-specific models and methods and (b) } \\
\text { the record of forum dialogue }\end{array}$ \\
\hline \multicolumn{2}{|r|}{$\begin{array}{l}\text { Question 1(a): What are the technologies, roles and other factors that help online CoPs to } \\
\text { embed knowledge? }\end{array}$} \\
\hline \multicolumn{2}{|c|}{ Technologies: } \\
\hline & $\begin{array}{l}\text { Online forums (facilitated identifying with others, piggybacking, alignment and } \\
\text { appropriation of key metaphors; exposed the thinking and emergent practices of } \\
\text { peers; fostered the discovery of like-minded people, leading to new relationships) }\end{array}$ \\
\hline & $\begin{array}{l}\text { Instant messaging (allowed discussions to be taken into invisible side-conversations } \\
\text { for problem solving; was used to solicit alignment) }\end{array}$ \\
\hline \multicolumn{2}{|l|}{ Roles: } \\
\hline & $\begin{array}{l}\text { Skilled online facilitation (building the community, selecting and seeding challenging } \\
\text { reframing content, and challenging thinking) }\end{array}$ \\
\hline & A sub-community of change agents (providing mutual support) \\
\hline & $\begin{array}{l}\text { Strong, change-focused workplace leadership, combined with a clear vision and } \\
\text { focus (and a requirement for online participation) }\end{array}$ \\
\hline \multicolumn{2}{|c|}{ Other factors: Buddying practices; Leading and following } \\
\hline \multicolumn{2}{|r|}{ Question 1(b): What is the nature of the knowledge embedding process in online CoPs? } \\
\hline & $\begin{array}{l}\text { Schools followed six discrete stages of a knowledge embedding cycle (KEC), or } \\
\text { professional change journey: Plotting the Course, Coming on Board, Setting Out, } \\
\text { Staying on Course, Anchoring and Settling. The embedding process was } \\
\text { characterised by differing activities at each stage. }\end{array}$ \\
\hline & $\begin{array}{l}\text { Sustained embedding of a new paradigm requires an embedding spiral in which } \\
\text { successive KECs enfold and reinforce the previous cycle(s) }\end{array}$ \\
\hline & $\begin{array}{l}\text { Online CoPs do not operate in isolation. Their value in facilitating the organisational } \\
\text { knowledge embedding process arises from the interplay of visible and invisible, } \\
\text { formal and informal, and online and offline dimensions of CoPs. }\end{array}$ \\
\hline
\end{tabular}

Table 21: Overview of how the meso-level theory answers the research question(s) 
At the micro, or individual, level, I have explained the embedding of knowledge as a gradual process of developing personal professional knowledge based around a combination of understandings (interpretive frameworks) beliefs and capability. According to my crossings theory, teachers can be seen as regularly operating in a situation of polycontextuality (Engeström et al., 1995), comprising different professional contexts or engagement spaces (such as the classroom, the staffroom, workshops, and the home, where they prepare lessons). They need to acquire, adapt, and apply knowledge as they cross between these spaces.

\begin{tabular}{|c|c|}
\hline & $\begin{array}{l}\text { evel theory: Overview } \\
\text { ting of personal professional knowledge was driven by individuals' crossings between } \\
\text { engagement spaces in a complex polycontextual system }\end{array}$ \\
\hline & $\begin{array}{l}\text { ion 1: How do online CoPs facilitate the transfer and embedding of professional } \\
\text { ledge? }\end{array}$ \\
\hline & $\begin{array}{l}\text { Online CoPs comprised multiple engagement spaces with distinct cultures of use. } \\
\text { Individuals made ongoing crossings between these spaces, and between online and } \\
\text { offline engagement spaces. When crossing, they had to translate, interpret, adapt, } \\
\text { combine, argue, analyse and justify ideas to fit the different cultures of use. This } \\
\text { facilitated embedding by enforcing focusing and adapting and by encouraging } \\
\text { persuading (blogs/forums). It resulted in owning (personalising) the new way and the } \\
\text { aligning of individuals with norms and with dominant themes. }\end{array}$ \\
\hline & $\begin{array}{l}\text { ion 1(a): What are the technologies, roles and other factors that help online CoPs to } \\
\text { d knowledge? }\end{array}$ \\
\hline & $\begin{array}{l}\text { ologies: Technologies were part of engagement spaces, linked with distinct cultures of } \\
\text { g. blogging culture promoted persuading). }\end{array}$ \\
\hline & $\begin{array}{l}\text { Roles may impact on the intensity/rate of embedding rather than the embedding } \\
\text { anism. For example, connector-leaders engaged with emerging ideas more regularly and } \\
\text { y online than others. (Refer to meso- and macro-level findings for dicussion of roles.) }\end{array}$ \\
\hline & factors: \\
\hline & $\begin{array}{l}\text { Engagement spaces: } \\
\text { Each engagement space had its own tools, genre and culture of use. These governed } \\
\text { agenda, language, tone, style, and register. }\end{array}$ \\
\hline & $\begin{array}{l}\text { Polycontextuality: } \\
\text { By providing multiple engagement spaces, online CoPs dramatically increased the } \\
\text { situation of professional polycontextuality. }\end{array}$ \\
\hline & $\begin{array}{l}\text { Polycontextuality: } \\
\text { By providing multiple engagement spaces, online CoPs dramatically increased the } \\
\text { situation of professional polycontextuality. }\end{array}$ \\
\hline & $\begin{array}{l}\text { Cultures of use: } \\
\text { Cultures of use linked with the engagement spaces enforced the continual adapting, } \\
\text { personalising and reinforcing of ideas. }\end{array}$ \\
\hline & ion 1(b): What is the nature of the knowledge embedding process in online CoPs? \\
\hline & $\begin{array}{l}\text { The embedding of knowledge was a continual process of knowledge personalisation, } \\
\text { driven by inter-contextual crossings. }\end{array}$ \\
\hline
\end{tabular}

Table 22: Overview of how the micro-level theory answers the research question(s) 
For the teachers who participated in the complex online CoPs in this study, the number of engagement spaces increased dramatically, creating a situation of hyperpolycontextuality. These people found themselves operating across multiple engagement spaces, bound up with distinct cultures of use, that required them to continually apply, adapt and personalise knowledge. Their ongoing crossings of the boundaries between these engagement spaces contributed powerfully to the embedding of the new paradigm, apparently acting as a catalyst. Table 22 provides a summary of how my micro-level theory addresses the research questions.

At the macro, or system of communities, level, I have explained the knowledge embedding process as having been facilitated by the interaction of different levels, or layers, of online communities, within an online CoP ecosystem. I have demonstrated how a middle layer boundary spanning community (CoP E) played a key role in this macro level embedding process, and how the roles of the connector-leader and follower-feeder played a key part in transferring and embedding knowledge. Table 23 provides a summary of how my macro-level theory addresses my research questions.

There is duality inherent in these three sets of findings: I have presented knowledge embedding as a process that occurs in stages at the meso level, but as a continual, stage-agnostic process when viewed from the micro and macro levels. This is consistent with a duality that I discovered in a review of the research literature prior to embarking on my study. Some researchers, including Szulanski (1996, 2000), Boisot (1998) and Kwan and Cheung (2006) have devised models presenting organisational knowledge transfer as a staged process. The benefit of such models is that they isolate issues and risks that pertain to different stages, highlighting relevant management problems and solutions. Other researchers have portrayed knowledge transfer as an ongoing process, based on a view of the organisation as a fundamentally social system. For example, Nonaka (1998) has highlighted the continual role that humans play in internalising, socialising knowledge, and in externalising it (making it explicit) and combining it; while Orlikowski (2002) has presented employees as engaged in a continual process of knowing.

I have come to see these two views as being complementary, or simultaneously true. The outcome of research into knowledge transfer depends not only on one's perspective and their level of analysis, but also on the way in which knowledge is viewed and the form that it takes. The creators of stage-based process theories (such as Szulanski, ibid) have studied the transfer of discrete items of organisational 
knowledge. Such research focuses on tracking the uptake of these items by the organisation over time. In a similar vein, my meso-level analysis investigated how CoPs facilitated the progressive embedding of the new way in schools, based around the introduction of school-specific teaching approaches, such as inquiry learning.

However, if one is studying the embedding of knowledge at a more abstract level, as was the case with Orlikowski's investigation of the evolution of workers' collective capability, the main concern is with the identification of ongoing processes. Similarly, my macro- and micro-level analyses aimed to identify how the new way, as a generic paradigm, was embedded. Rather than tracking the uptake of a specific knowledge object, these perspectives account for the embedding of a broad set of related ideas and themes. 


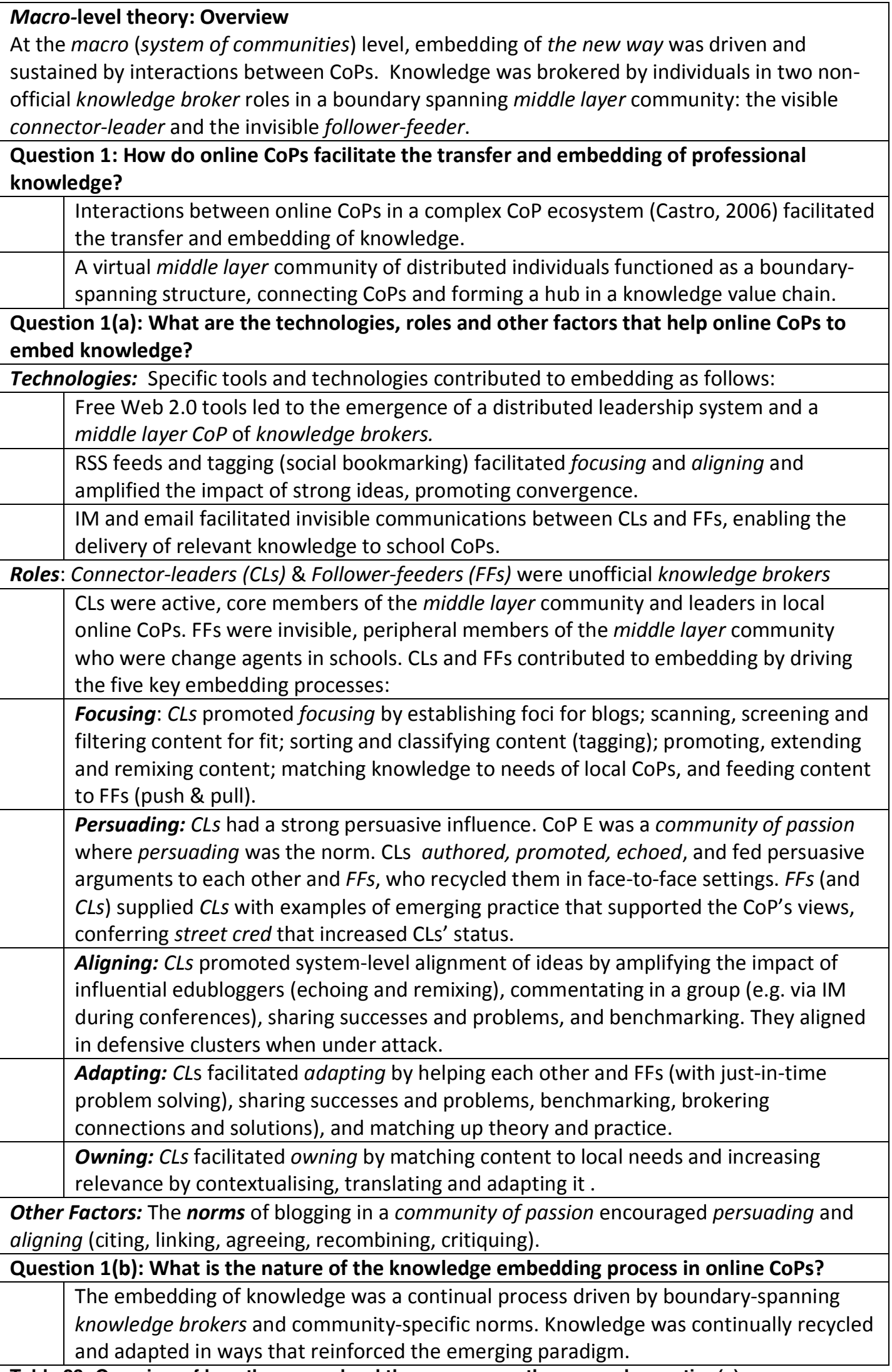

Table 23: Overview of how the macro-level theory answers the research question(s) 


\subsection{Limitations}

The key limitation of this research project is that, as is typical of case-based research, the results are strongly contextual. They are therefore unable to be readily transferred to other contexts. As interpretivist theory, the results outlined here represent my subjective interpretation of events and their significance. They also need to be seen as part of larger set of more complex events that occurred in reality. Those who seek parsimony and simplicity in IS theory would see these factors as limitations.

A further limitation arises from the fact that my understanding of teachers' emerging knowledge and how it impacted on their understandings and classroom practice was based largely on their subjective, retrospective reports and recollections. It is not possible to know how closely their reports resembled what actually occurred, but it is likely that hindsight led to some 'collapsing' of events. For example, in interviews, some teachers recalled having made a rapid decision to come on board, but facilitators noted that in their experience, making a decision with this speed had been rare. (Research by Wagenaar (1986) has shown that people's memories can be highly unreliable when it comes to orientating events in time). I aimed to infer the sequence of events by matching up teachers' verbal recollections of events with time-specific online data in forums and/or blogs. However, it was often difficult to establish the exact sequence of events. There is a related risk in some schools and/or teachers may have exaggerated the extent of embedding of the new way that had occurred, with the aim of appearing to be progressive.

I have noted in Chapter 7 that there was, to some extent, a meso-level bias in the views of knowledge embedding provided to me by my research participants. Their focus on change that had occurred at the organisational level appears to be due, in part, to the large proportion of school leaders and change agents in my sample. This undoubtedly arose from my need for schools to select individuals who were participants in online CoPs; and perhaps from schools' desire to promote the favoured institutional view. More imaginative understanding (Charmaz, 2006, p.126) was required from me as a researcher when investigating the micro- and macro-level facilitating mechanisms. I compensated for this imaginative input by conducting thorough analyses of online records and by triangulating online and offline records.

CoPs in which the embedding of new professional knowledge was seen to have been successful formed the basis of my research case. While this was necessary in order to 
answer my how question, it can be seen as providing limitations in terms of my exposure to the barriers operating against the phenomenon I investigated.

Nonetheless, even in the selected research context, I was able to identify some key barriers to the effective utilisation of online communities to support professional change via knowledge embedding.

\subsection{Relationship of key findings to existing theory}

Table 24 relates my findings to the previous 'baseline' position, as established in the literature summary (see chapter two, page 55). It provides a detailed summary of the relationship between my major findings at each level and existing theory (or gaps in the body of theory). I discuss the key theoretical relationships in the implications section that follows.

\subsection{Implications of this study}

This study has a number of implications for practice and research. I first consider its general implications and then discuss specific implications that arise from the meso-, macro-, and micro-level findings.

\subsubsection{Potential of online CoPs to drive professional change}

This study suggests there is potential to use online CoPs as a tool to help drive professional change - not merely to promote the acquisition of new procedural methods, but to foster a deeper change in the way in which professionals conceive of their role and its source of value. In this study context, this influenced professionals' motivation to enact and sustain meaningful change in their practice. Online CoPs appear to be well suited for supporting change strategies that take an empirical rational and/or normative-re-educative approach (Chin and Benne, 1969).

The empirical-rational (ER) approach drives change through understanding and reasoning. It relies on convincing people of the value of change. The online CoPs in this study facilitated ER change by (a) promoting focusing on change-related themes, (b) facilitating persuading through the seeding and cultivation of powerful themes, (c) promoting theory-practice crossings in which teachers interrogated their practice in light of new ideas and (d) fostering owning of an emerging paradigm. 


\begin{tabular}{|c|c|}
\hline \multicolumn{2}{|c|}{ Relationship of key findings to existing theory } \\
\hline \multirow[t]{2}{*}{ Overview } & $\begin{array}{l}\text { Shows how macro- and micro-level embedding processes operate concurrently } \\
\text { with organisational level processes. Suggests the potential for multi-level } \\
\text { research that investigates interdependencies of these processes. }\end{array}$ \\
\hline & $\begin{array}{l}\text { Demonstrates a duality in the knowledge embedding process, helping explain } \\
\text { the tension in KM literature between an ongoing process view (e.g., Orlikowski, } \\
\text { 2002, Nonaka, 1998) and a staged view (eg. Szulanski, 2000). In this study, the } \\
\text { staged view accounts for the embedding of specific knowledge (models, or } \\
\text { boundary objects) in schools, whereas the continual process view illustrates the } \\
\text { progressive embedding of a larger paradigm at the individual and system level. }\end{array}$ \\
\hline \multirow[t]{3}{*}{$\begin{array}{l}\text { Meso } \\
\text { view }\end{array}$} & $\begin{array}{l}\text { Builds on research into organisational knowledge transfer by focusing on a key } \\
\text { aspect of knowledge transfer; the knowledge embedding process. This has been } \\
\text { identified as a key gap in KM (Argote et al., 2003). }\end{array}$ \\
\hline & $\begin{array}{l}\text { Identifies a model for knowledge embedding, the Knowledge Embedding Cycle. } \\
\text { Unlike linear models of knowledge transfer, the KEC shows embedding of new } \\
\text { paradigmatic knowledge as being staged, but cyclic and iterative. }\end{array}$ \\
\hline & $\begin{array}{l}\text { Provides a context-specific view of the evolution of embedded (or sticky) } \\
\text { knowledge in an organisation. Previous efforts have focused on identifying how } \\
\text { to unstick sticky knowledge (Szulanski, 2000) }\end{array}$ \\
\hline \multirow[t]{4}{*}{$\begin{array}{l}\text { Micro } \\
\text { view }\end{array}$} & $\begin{array}{l}\text { Builds on research positioning knowledge transfer as driven by boundary } \\
\text { crossing (Ancona and Caldwell, 1992, Levina and Vaast, 2005, Pawlowski and } \\
\text { Robey, 2004). Identifies a new type of boundary, between engagement spaces, } \\
\text { within online CoPs. Illustrates how ongoing crossings between engagement } \\
\text { spaces can lead to the embedding of personal knowledge. }\end{array}$ \\
\hline & $\begin{array}{l}\text { Builds on research into expert cognition, extending the concept of professional } \\
\text { polycontextuality (Engeström et al., 1995, Goodwin, 1990, Reder, 1993). Online } \\
\text { CoPs dramatically increased the situation of polycontextuality faced by change } \\
\text { agents, leading to hyper-polycontextuality, which in turn promoted embedding. }\end{array}$ \\
\hline & $\begin{array}{l}\text { The specificity of the individual-level embedding mechanism underlines the } \\
\text { importance of Personal KM; an under-researched area of KM (Pauleen, 2009). }\end{array}$ \\
\hline & $\begin{array}{l}\text { Updates research into online CoPs by presenting the CoP as a complex, } \\
\text { polycontextual environment rather than a single shared space. This can be seen } \\
\text { as an extension of Castro's }(2004,2006) \text { online CoP ecosystem. }\end{array}$ \\
\hline \multirow[t]{3}{*}{$\begin{array}{l}\text { Macro } \\
\text { view }\end{array}$} & $\begin{array}{l}\text { Updates research into online communities by investigating the workings of a } \\
\text { complex online CoP ecosystem (Castro, ibid) that includes a blogging community } \\
\text { and considers its interaction with forum-centric facilitated CoPs. Illustrates how } \\
\text { knowledge flows within this complex, tiered online CoP system. }\end{array}$ \\
\hline & $\begin{array}{l}\text { Introduces the concept of a middle layer knowledge broking community, } \\
\text { elevating the concept of boundary crossing to a structural level. }\end{array}$ \\
\hline & $\begin{array}{l}\text { Enriches the existing body of research into the role of the knowledge broker in } \\
\text { knowledge transfer, identifying two new roles, the Connector Leader (CL) and } \\
\text { Follower-Feeder (FF). Identifies a series of knowledge embedding practices } \\
\text { performed by online knowledge brokers and challenges the traditional concept } \\
\text { of the lurker (Nonnecke et al., 2006, Rafaeli et al., 2004, Soroka and Rafaeli, } \\
2006 \text { ) as being an unproductive member of online CoP. }\end{array}$ \\
\hline
\end{tabular}

Table 24: Relationship of key findings to existing theory 
The normative-re-educative (NR) approach is based on accomplishing change through cultural and social means. It aims to establish new norms and values and foster commitment to these. The online CoPs in this study supported NR change by (a) helping establish new communication norms, (b) facilitating persuading (leading to questioning of traditional values and normalising of the new student-centred approach), (c) promoting the ongoing aligning of interpretive frameworks and beliefs amongst individuals, and (d) facilitating a culture of just-in-time professional support.

In this study, the rational and empirical aspects of change were deeply intertwined, largely owing to the brokering role of $\mathrm{CoP} \mathrm{E}$, a community of passion, whose values and beliefs were inseparable from the knowledge they circulated amongst CoPs.

\section{Implications for practice:}

If online communities are to successfully drive change, the approach taken should fit with one, or both, these types of change strategy. (It would seem inappropriate to try to use online CoPs to drive power-coercive change.)

\section{Implications for research:}

Online collaboration tools have previously been seen as suitable for supporting the sharing and incremental refinement of knowledge (Wagner and Bolloju, 2005). This study indicates a greater potential for use of these tools by a CoP, if effectively managed and combined with a clear sense of focus and vision. There is an opportunity for future research to explore the suitability of online CoPs to drive ER and NR change, and clarify how to best design CoPs for each approach.

\subsubsection{Understanding how knowledge is embedded}

Thus study has extended KM research by exploring the process of knowledge embedding, or deep knowledge transfer, in the context of professional change. It has developed a multi-level theory of how embedding was facilitated by online CoPs and has isolated five fundamental processes - focusing, persuading, aligning, adapting and owning - that promoted the embedding of transformative knowledge. These processes were driven by embedding-facilitating mechanisms at all three levels of analysis meso, micro and macro. 


\section{Implications for practice:}

The five processes could be used as a tool to guide and streamline the application of online CoPs where professional change is the goal. Although online CoPs were complex, with multiple dimensions, technologies and cultures of use, the embedding processes played a constant, easy-to-understand role in promoting embedding.

In the study context, the MOE had placed an emphasis on using evidence to drive professional change. However, teachers' explicit use of evidence played little part in the embedding process, which relied more strongly on persuasion. The embedding of evidence-based knowledge may be best accomplished through the seeding of powerful content (based on evidence) and recruiting appropriate people as influencers.

\section{Implications for research:}

The five knowledge embedding processes could be tested in other contexts to determine their transferability.

\subsubsection{Multi-level theory of knowledge embedding}

This study identified three complementary knowledge embedding facilitating mechanisms that were simultaneously operating at the meso, micro and macro levels of the research case.

\section{Implications for practice:}

This suggests an opportunity to utilise online CoPs in a targeted way to promote professional change at any, or all, of these levels. This is of particular relevance to those planning change at industry level, and to those such as government whose objective is system-level change. The study highlights the need for stakeholders to consider the level(s) of the system at which embedding is required and to manage accordingly. If taking a whole-of-system perspective it is necessary to recognise that the mechanisms operating at different levels are complementary and that interdependencies are involved: For example, the ability of online CoPs to sustain change at macro (system) level relied on the brokering activities of people who play meso-level leadership roles in organisations involved in fixed-term change programmes. Issues arise from the fact that the macro-level value provided by these people may not be recognised by their employers, and that the funding models designed to support fixed-term organisational change cannot support longer-term macro-level brokerage. Whether these problems are offset by the reputational gains made by individuals in their informal system-level leadership roles is unclear. 
The need for strong leadership, and other facilitating factors outside the scope of this study, should not be overlooked.

\section{Implications for research:}

Applying a multi-level approach is a suitable way of acknowledging the complexity involved in the context of transformative professional change: A single theoretical perspective can provide only a partial explanation for a complex phenomenon (Edwards, 2005, p.269). The multi-level view does, however, raise questions regarding the interdependencies of mechanisms operating at different levels. It was beyond the scope of this study to investigate cross-level interdependencies, but future studies could do this. For example, there is an opportunity to consider in detail how individual (personal) knowledge is embedded in the organisation (becoming organisational knowledge) and organisational knowledge is embedded in individuals (becoming personal knowledge). Zhang (2009) has proposed a framework for these reciprocal processes of personalising and organisationalising.

\subsubsection{Knowledge embedding as a staged and an ongoing process}

This study suggests that it is useful to view the process of embedding knowledge as both a staged and an ongoing process: The meso-level KEC was a stage-based model, but also a spiral. The embedding facilitating mechanisms operating at micro and macro levels were continuous processes.

\section{Implications for practice:}

Where the intent is whole-of-organisation alignment and change, it is useful to view embedding as a staged process. However, it is also useful for managers to take a micro-level view, recognising that staff have individual needs: They need opportunities to apply, adapt and personalise new knowledge in the company of like-minded peers. Likewise, appreciation of the ongoing nature of macro-level knowledge-brokering activities would help managers understand the value provided by knowledge brokers.

\section{Implications for research:}

The three-level theory casts a new light on the apparent dichotomy in the literature, illustrating that it is useful to view KT and embedding as being simultaneously a stagebased and an ongoing processes. The perspective taken is linked with the intended outcomes and the level of analysis (e.g., anchoring of a whole-of-school approach versus building deep personal understanding). It would be useful for researchers to be aware of the value of both perspectives when undertaking research in this area. 


\subsubsection{Underestimating the value and complexity of online CoPs}

In this study, the value and complexity of CoPs went unrecognised by many stakeholders. This was partly because many participants conceived of online CoPs as discrete online spaces, and partly because they were unable to see the invisible dimension of online CoP activities that accounted for much of their KT and transformative power. This study uncovered a significant amount of invisible online activity amongst community members, often undertaken via IM tools. This invisible activity helped to drive and embed professional change, and to influence other, more visible, online activities, as well as events in the workplace.

The study also exposed a complex reality in which online CoPs formed part of a polycontextual socio-technological system: Diverse technologies were bound up with distinct cultures of use, and knowledge continually flowed between online and offline, formal and informal, and visible and invisible engagement spaces. The crossing of ideas, beliefs and norms between online and offline engagement spaces promoted individual transformation, yet was invisible. Likewise, the side-conversations that promoted collegial aligning and KT between connector-leaders and follower-feeders were invisible, as were the online-offline brokering activities of follower-feeders.

\section{Implications for practice:}

These findings demonstrate a need for organisations to develop a more complex conception of online CoPs; one that recognises their significance as part of a multiplicity of professional engagement spaces and therefore allows for their role in facilitating online-offline interactions. This in turn should foster valuing of the roles of key players. Practitioners and researchers alike would benefit from an awareness of the invisible dimension of CoP activity. The aim should not be to monitor this invisible activity, but to be aware of it, and foster it so that it supports community needs.

\section{Implications for research:}

My conceptions about online CoPs were challenged by my discovery of an active online CoP of bloggers in this study. Recognition of this CoP led me to richer findings than would otherwise have been the case. This illustrates how vital it is for researchers to recognise the complex and diverse nature of contemporary online CoPs and reflect this in their studies. In particular, it is important to consider CoPs' use of diverse tools (which I suggest are best conceived of as engagement spaces) and to recognise the existence of overlapping CoPs. 
This study also demonstrates the value of taking a holistic view of online CoPs. For example, when CoPs have both online and offline dimensions, the value of the online dimension can only be understood by considering how it operates in relationship to the offline dimension. Likewise, the value of the visible dimension of online CoP activity is best understood by considering its relationship to the invisible dimension. A surprising number of studies view online CoPs as if they exist in total isolation from other contexts. Even in the case of virtual communities (such as CoP E) there are likely to be interactions with other CoPs. By acknowledging this kind complexity, future studies of online CoPs may lead to a richer understanding of CoP dynamics.

\subsubsection{Emerging ecological themes}

This study has identified a number of ecological themes that are worthy of exploration: The online CoP environment exemplified Castro's online CoP ecosystem. Within this ecosystem, connector-leaders and follower-feeders played symbiotic roles, as did thinker-doer buddies. Knowledge was recycled and recombined as it was passed from primary consumers (connector-leaders) to secondary consumers (follower-feeders) and those who 'fed' off them (regular teachers). This was reminiscent of a food chain or web. By applying an ecological lens, future interpretive studies may uncover new insights into the workings of online CoPs.

This study also suggests an opportunity to research the theme of succession in relationship to the development of an online CoP ecosystem. Succession is the gradual supplanting of a plant or animal community by another. (In NZ, manuka trees can flourish on bare soil, providing a nursery environment in which forest seedlings are protected as they grow. As they mature, the forest trees take over.) In the ICT PD clusters, formal online CoPs provided finite value - they were a powerful means of bringing staff on board, uniting interpretive frameworks and establishing new cultural norms. However, the sustaining of change appeared to be dependent on a shift on the part of key participants towards using more informal, distributed CoPs within online networks. The timing of the transition to this informal system may be critical in order to build on the success of local CoPs before they have outlived their purpose. The ecological theme of succession could be a useful framework to apply in studies.

\subsubsection{General theoretical implications}

The study resulted in a three-level explanatory theory. Although explanatory theory does not aim to predict and or produce testable propositions (Gregor, 2006, p.630) it 
can be a useful input into two other types of IS theory: theory for explaining and predicting and theory for design and action (ibid). Future studies could build on my explanatory findings to develop more transferable and predictive theory. For example, the KEC could be developed into a predictive tool for by testing it in other settings (perhaps using action research) and my theory about how polycontextuality promotes personal embedding of knowledge in online CoPs could be further explored and tested.

\subsubsection{General methodological implications}

Some of my research methods may be of value to future research. My use of diverse data sources (combining interviews with analysis of online data) proved valuable in exposing the significant invisible dimension of online CoPs. Without combining and triangulating these rich data sources the results would have been considerably less rich: The invisible dimension of online activity, the micro-level crossings mechanism, and the online-offline brokering practices of follower-feeders would have remained invisible. With the expansion in IM there is a significant opportunity for future studies to explore the interaction of the visible/invisible duality of online CoPs in more depth. Gaining greater access to IM records would facilitate this.

My analysis of the development of discrete themes over time as they crossed engagement spaces was a valuable way of tracking KT and embedding. Future studies could extend this approach; for example, tracking the system-level transfer and embedding of a single theme within a finite time period. Finally, my use of an iteratively developed interpretive toolkit may be of interest to other interpretive researchers.

I now consider implications of the meso-, micro- and macro-level findings.

\subsubsection{Implications of the meso-level findings}

At the meso (organisational) level, the embedding of knowledge followed a six-stage knowledge embedding cycle (KEC). Different issues and activities characterised each stage of the cycle, with online CoPs playing a changing role.

\section{Implications for practice}

Subject to further testing, the KEC could become a useful framework for managers, promoting an awareness of stage-dependent issues and guiding their application of online CoPs. Coming on Board was supported by an official, facilitated online 
community. There was a shift to a dependence on a non-official, distributed online CoP to support Staying on Course. During Anchoring, the official CoP was again needed to aggregate school-specific knowledge. This suggests the need for managers to cultivate the kind of online environment that suits their organisation's state and stage of knowledge. It may be useful to alternate the periodic reinvigoration of official online communities with the recruitment of individuals to more informal, distributed communities. It seems there is also a need to balance inward-facing and outwardfacing roles.

Another key issue is the problem of the sustainability of online communities. To truly embed a new teaching paradigm, a knowledge embedding spiral was seen by some participants as being necessary, with each successive cycle reinforcing the previous one. In the schools and clusters studied, one KEC had taken three years to complete, by which time schools' funding for an online facilitator had been exhausted. As schools embedded a specific teaching approach, the need for a centralised online community diminished, and the need for individualised support increased. This support was provided largely met by those in change agent and unofficial leadership roles, often in the evening, via their connections with the distributed online community, CoP E. However, the availability of funded release time from classroom duties remained an enabler of online participation, and at least some of the holders of voluntary roles had some form of programme-related government funding. It is unclear whether the distributed online CoP system would be sustainable without this funding. In addition, for schools to bring reluctant practitioners on board would require a second cycle, but it is unclear whether this could be achieved without the official online community. Given staff turnover and the need for ongoing KECs, it would seem pragmatic for organisations to aim to retain an official, facilitated online community.

\section{Implications for research:}

Previous models of KT employ a linear model with a fixed endpoint, but key research participants suggested that a spiral model - comprising successive KECs - was more suitable for explaining the process of sustained embedding. It would be beneficial to test the KEC model in other contexts and refine it (for example, using action research), with the aim of developing it into a management tool. It would also be useful to conduct longitudinal research into knowledge embedding to ascertain the applicability of the spiral model over, perhaps, a 5-10 year period. 


\subsubsection{Implications of the micro-level findings}

My micro-level findings illustrate how individuals' participation in diverse engagement spaces promoted personalising and owning though the continual need to adapt and recontextualise knowledge.

\section{Implications for practice:}

Managers would benefit from recognising the totality of online and offline, formal and informal, and visible and invisible interactions in embedding new professional knowledge. (Such a holistic view of community was evident to very few of those interviewed, with the result that the value and impact of the online dimension of CoPs was poorly recognised.) Managers should encourage the shared use of a range of complementary tools and ensure that $\mathrm{CoP}$ members understand the value of different engagement spaces. (For example, the cultures of use linked with blogs and forums encourage the matching up theory and practice, while IM culture encourages mutual alignment.)

Today's online CoPs are operating in a more complex environment than early generation CoPs. The uptake of user-centred Web 2.0 collaboration tools, and the resulting distributed nature of conversations, can be seen as dramatically enhancing the situations of polycontextuality and boundary crossing that professionals have historically faced (Engeström et al. 1995, Goodwin 1990; Reder 1993). It is possible that this enhanced polycontextuality, when leveraged appropriately by a changefocused community, has the potential to facilitate deeper professional knowledge transfer than might otherwise occur in situations where individuals have little sustained face-to-face contact. Further study is needed to explore this possibility.

\section{Implications for research:}

My theory at this level is a novel contribution to IS research. I have combined the concept of boundary crossing (Ancona \& Caldwell, 1992; Levina \& Vaast, 2005; Pawlowski \& Robey, 2004) with the concept of polycontextuality from studies of expert cognition (Engeström et al., 1995; Goodwin, 1990; Reder, 1993) to explain how knowledge embedding was facilitated at an individual level for active members of online CoPs. The specificity of the knowledge-embedding mechanism at this level underlines the importance of Personal KM, an under-researched area (Pauleen, 2009). 
Rather than viewing online CoPs as a group of users using different tools, I have conceived of the CoP as a set of diverse professional engagement spaces in a sociotechnological system. This system was characterised by multiple dualities of context and governed by cultures of use (arising from human-technology interaction) that placed contextual demands on users. The study identifies a new type of boundary: the boundary between engagement spaces in online CoPs. When individuals undertook crossings between these spaces they needed to adapt, recontextualise and personalise knowledge to meet these demands (e.g., using themes in an original way, providing evidence, justifying a stance with theory). This stimulated deeper thinking, building personal understanding that led to owning of knowledge. In the CoPs I studied, there had been a dramatic expansion in the number of engagement spaces, leading to a situation of (hyper) polycontextuality and resulting in more frequent crossings than before. This seems to have acted as a catalyst for personal knowledge embedding.

Situated learning theory (Lave and Wenger, 1991) underpins early studies of CoPs, but cannot account for knowledge embedding in polycontextual CoPs, where participants are constantly becoming re-situated. My crossings theory provides an alternative explanation for how knowledge is embedded by individuals in such contexts: The individual professional is a boundary spanner in a complex CoP environment made up of multiple engagement spaces. This emergent theory represents only part of my findings and requires further exploration. Future investigation of crossings would ideally be conducted in real-world settings. A useful approach would be to focus on tracking specific ideas and themes as they are embedded over time. Data from visible and invisible online sources should be included and triangulated with interviews and perhaps observations.

\subsubsection{Implications of the macro-level findings}

At the macro level, the study illustrates the workings of a complex multi-layered online community, or online CoP ecosystem (Castro, 2004, 2006). This system comprised formal, facilitated, closed, forum-based communities and an open, informal online community based around Web 2.0 technologies. The macro-level findings extend the theme of boundary spanning in $\mathrm{KM}$, identifying a middle layer community as playing a key knowledge-brokering function. I identified two types of boundary spanner in this community who played complementary roles: the visible connector-leader (spanning online boundaries) and the invisible follower-feeder (spanning the online-offline boundary). Connector-leaders played non-official, but critical, roles in brokering knowledge. The activities were sophisticated and their skills and understanding had 
been built over time. They also had natural connector and leader attributes. Followerfeeders were knowledge brokers who helped drive change by bringing online community knowledge into the workplace. Their activities resulted in online communities having an indirect influence on the so-called regular teacher.

\section{Implications for practice:}

It is not feasible for managers to aim to build a complex online CoP structure from scratch. It would be better to seek out and leverage existing online CoPs and foster a connecting layer, or sub-group of connector-leaders. Similarly, connector-leaders cannot easily be 'made'. It is better to identify and recruit people with appropriate attributes, connections, skills and knowledge. It is also necessary to ascertain how these intermediary roles should be recognised, funded and sustained.

The fact that CoP E was a community of passion was a key finding: Individuals who care about the ideas they are passing on are likely to be more effective than those who do not. Managers should encourage those with passion for an emerging professional approach to connect with like-minded others. They should also encourage internal change agents to communicate with suitable online influencers and allow time for this.

There was under-recognition of the value of connector-leaders and follower-feeders; knowledge brokers who operated across system boundaries. Some connector-leaders expressed feeling undervalued. This is unsurprising, given that schools have no responsibility for system-level change and traditional staffing structures do not support such roles. It is unclear how best to support these broker roles, but if online communities are to play a meaningful part in promoting knowledge embedding, existing staffing models and ways of recognising value will need to be replaced with new models and means of recognising the value of brokering activities.

The invisibility of the follower-feeder role can be seen as presenting a barrier to gaining ongoing stakeholder support to online CoP initiatives. In this study, some people in leadership positions portrayed connector-leaders as likeable, albeit socially deprived eccentrics, whose long hours of extramural online activity had little relevance to the regular teacher. Upon reflection, this is unsurprising: The true value of connectorleaders could only be seen when the role of follower-feeders was taken into account, yet the invisibility of follower-feeders' interactions with connector-leaders made this value impossible to recognise. Taking a system perspective is undoubtedly difficult for 
those inside the system, but failure to do so could lead to the kind of problem that occurs if a key species is removed from a functioning ecology.

\section{Implications for research:}

Connector-leaders spanned the boundaries of two or more online communities and were members of an international network. They were therefore in a position to combine the value of CoPs and networks. Future research could consider in depth how

such knowledge brokers operate and the benefits they provide. Connector-leaders had different individual attributes (for example, some were natural thinkers while others were doers). There is an opportunity for future research to consider the workings and make-up of such a distributed community in more depth. Perhaps, for example, there is a desirable degree of diversity, an identifiable range of complementary facilitating attributes, or an optimum number of members.

The discovery of the follower-feeder's critical role is significant because it challenges the somewhat simplistic lurker concept (Nonnecke et al., 2006; Nonnecke \& Preece, 1999; Nonnecke et al., 2004; Rafaeli et al., 2004; Soroka \& Rafaeli, 2006). Existing studies acknowledge that little is known about so-called lurkers. My findings help to address this gap. Follower-feeders did not lurk: They were inward-facing knowledge brokers who spanned the online-offline CoP boundary, feeding knowledge they had gained online from following connector-leaders to regular teachers in offline settings. Follower-feeders were reluctant to leave visible traces in the public online record (e.g., on blogs), communicating instead using invisible means, such as IM. This meant that the true nature of their role was invisible to managers; as was the role of connectorleaders in providing them with knowledge. Assumptions about the lack of value provided by the so-called lurker may arise from a lack of recognition of what I have termed the online-offline CoP boundary, and the need to broker knowledge across it.

\subsection{Conclusion}

This interpretive case research project used qualitative methods to investigate how online CoPs facilitated the transfer and embedding of professional knowledge in the context of transformative change. It was motivated by a lack of understanding about how online CoPs work and how 'deep' knowledge transfer, or embedding, occurs. The study also aimed to address a real-world problem: The NZ government had embarked on a high-level strategy of embedding knowledge about effective teaching in the schooling system. Aiming to leverage its investment in IT infrastructure, it had placed 
an onus on online CoPs to help achieve this. What was missing was a clear understanding of how this might occur, and therefore how to facilitate the process.

The study context was a unique case; a national ICT PD programme for schools. I analysed data from five online CoPs associated with this programme, taking a multilevel perspective. The result is a three-level explanatory theory: I found that online CoPs facilitated knowledge embedding via complementary embedding-facilitating mechanisms operating at the meso, micro and macro levels. These mechanisms helped drive five fundamental knowledge embedding processes: focusing, persuading, aligning, adapting and owning.

While subject to the usual limitations of interpretive case research, this study has helped address the identified gaps in the literature. It has produced a rich contextual understanding of how knowledge embedding occurred in the research setting, and how online CoPs facilitated this. It has also produced the following insights:

- The study illustrates the complexity of a contemporary online CoP and the value of this complexity: The CoPs studied were part of a larger ecosystem comprising overlapping structures and multiple engagement spaces. This ecosystem provided a multiplicity of boundaries across which knowledge was continuously transferred. When combined with the knowledge-brokering activities of a community of passion, employing sophisticated cultures of use so as to exploit the benefits of different technologies, this ecosystem enabled the recycling and amplification of powerful themes, facilitating both local-level ownership and system-level convergence. The polycontextuality of the new professional environment acted as a catalyst for individuals to personalise and embed the new paradigm, modifying their professional beliefs and identities.

- The study has exposed the importance of various dualities in the knowledge embedding process: Embedding was both a staged and a continuous process. CoP members personalised knowledge as they crossed between visible and invisible, and formal and informal, engagement spaces, iteratively matching up theory and practice. New communication norms were established online, then crossed into the offline workplace, setting up a virtuous cycle that helped drive change. Connector-leaders and follower-feeders played symbiotic roles, helping to transfer knowledge across the online-offline boundary. 
- My findings suggest that there is a significant invisible dimension in today's online CoPs. Although activities based around IM, Twitter and email are easily overlooked, they may play a key role. In this study, the use of invisible engagement spaces facilitated deeper thinking and the alignment of perspectives, fostering acceptance of change, adaptation and perseverance.

- Boundary spanning has been previously identified as a promoter of knowledge transfer. This study identifies three new kinds of boundaries: the boundaries between engagement spaces, the concept of a boundary-spanning middle layer community, and the online-offline CoP boundary. The online-offline boundary is so important to organisational knowledge transfer that online CoPs may be better conceived of as the online dimension of CoPs, unless they are entirely virtual.

- The study suggests a strong potential for online CoPs to be used as a tool to support professional change strategies that use a normative-re-educative and/or empirical-rational approach. However, it has also exposed a lack of understanding of the potential (and actual) value of online CoPs. Key stakeholders cannot readily see the holistic view of online CoP activity. This was linked with an undervaluing of those who play key knowledge broker roles. Compounding this problem, traditional staffing and funding structures do not cater for the sustaining of system-level knowledge flows.

In summary, this study has aimed to add richness to IS and KM research by presenting a multi-level view of how online CoPs drove the process of knowledge embedding, or deep transfer, in the context of professional change. I believe there are abundant opportunities for future research that takes a system perspective, particularly with relationship to driving system-level reform initiatives.

In this chapter I have summarised my key findings and discussed their relationship to the existing body of theory. I have also considered the potential value of these findings to practitioners and researchers and suggested possible opportunities for future research. I hope that this contribution will be of future use to the IS community and other stakeholders. 


\section{BIBLIOGRAPHY}

Albino, V., Garavelli, A., \& Gorgoglione, M. (2004). Organization and Technology in Knowledge Transfer. Benchmarking: An International Journal, 11(6), 584-600.

Allee, V. (2000). Knowledge Networks and Communities of Practice. OD Practitioner, 32(4).

Allen, T. (1967). Communications in the Research and Development Laboratory. Technology Review, 70, 31-37.

Allen, T. (1977). Managing the Flow of Technology: Technology Transfer and the Dissemination of Technological Information within the $R \& D$ Organization. Cambridge, MA: MIT Press.

Alton-Lee, A. (2003). Quality Teaching for Diverse Students in Schooling: Best Evidence Synthesis. Wellington, NZ: Ministry of Education.

Alton-Lee, A. (2006, March 29, 2006). Iterative Best Evidence Synthesis Programme: Strengthening Research, Policy and Practice Links to Improve Outcomes. Paper presented at the 4th Annual Policy Conference: Policy Evolution. Retrieved from http://www.educationcounts.govt.nz/themes/BES.

Ancona, D., \& Caldwell, D. (1992). Bringing the Boundary: External Activity and Performance in Organizational Teams. Administrative Science Quarterly, 34(4), 634-665.

Applebee, A. C., Ellis, R. A., \& Sheely, S. D. (2004). Developing a Blended Learning Community at the University of Sydney:

Broadening the Comfort Zone. In R. Atkinson, D. McBeath, D. Jonas-Dwyer \& R. Phillips (Eds.), Beyond the Comfort Zone: Proceedings of the 21st ASCILITE Conference, Perth (pp. 58-66).

Ardichvili, A., Page, V., \& Wentling, T. (2003). Motivation and Barriers to Participation in Virtual Knowledge-Sharing Communities of Practice. Journal of Knowledge Management 7(1), 64-77.

Argote, L., \& Ingram, P. (2000). Knowledge Transfer: A Basis for Competitive Advantage in Firms. Organizational Behaviour and Human Decision Processes, 82(1), 150-169.

Argote, L., McEvily, B., \& Reagans, R. (2003). Introduction to the Special Issue on Managing Knowledge in Organizations: Creating, Retaining, and Transferring Knowledge. Management Science, 49(4), v-viii.

Argote, L., \& Ophir, R. (2000). Learning Within Organizations: Factors Affecting the Creation, Retention, and Transfer of Knowledge. In J. Baum (Ed.), Blackwell Companion to Organization. Oxford: Blackwell.

Argyris, C., \& Schön, D. (1978). Organizational Learning: A Theory of Action Perspective. Reading, Mass: Addison Wesley.

Armistead, C. (1999). Knowledge Management and Process Performance. Journal of Knowledge Management, 3(2), 143-154.

Badaracco, J. (1991). Alliances Speed Knowledge Transfer. Planning Review, 19(2), 10-12.

Baker-Eveleth, L., Sarker, S., \& Eveleth, D. (2005). Formation of an Online Community of Practice: An Inductive Study of Key Elements. Proceedings of the 38th Hawaii International Conference on System Sciences (HICSS '05), 8, 254b. Available at http://doi.ieeecomputersociety.org/210.1109/HICSS.2005.1275.

Barney, J. (1991). Firm Resources and Sustained Competitive Advantage. Journal of Management, 17(Special Issue), 99-120.

Barney, J., Wright, M., \& Ketchen, D. (2001). The Resource-Based Theory of the Firm: Ten Years After 1991. Journal of Management, 27(Special Issue), 625-641.

Bateson, G. (1972). Steps to an Ecology of Mind. New York: Ballentine. 
Bell, M., Cordingley, P., \& Mitchell, H. (2006). The Impact of Networks on Pupils, Practitioners, Organisations and the Communities they Serve: A Summary of the Systematic Review of the Literature: National College for School Leadership (NCSL), England. Retrieved from http://www.ncsl.org.uk/mediastore/image2/the-impact-of-networks-on-pupilspractitioners-organisations-and-the-communities-they-serve.pdf.

Bennett, N. (1992). Perspectives on Knowledge Bases for Teaching. In T. Plomp (Ed.), European Conference on Educational Research (pp. 339-342). Enschede, The Netherlands: University of Twente.

Bereiter, C. (2002). Education and Mind in the Knowledge Age. Mahwah, NJ: Lawrence Erlbaum Associates.

Bhaskar, R. A. (1979). The Possibility of Naturalism. London: Routledge.

Boisot, M. H. (1998). Knowledge Assets: Securing Competitive Advantage in the Information Economy. Oxford: Oxford University Press.

Bonk, C., \& Kim, K. (1998). Extending Sociocultural Theory To Adult Learning. In M. Smith \& T. Pourchot (Eds.), Adult Learning And Development: Perspectives From Educational Psychology (pp. 67-88). Mahwah, NJ: Lawrence Erlbaum

Borko, H., \& Putnam, R. (1996). Learning To Teach. In D. Berliner \& R. Calfee (Eds.), Handbook of Educational Psychology (pp. 673-708). New York: Macmillan.

Bromme, R., \& Tillema, H. (1995). Fusing Experience and Theory: The Structure of Professional Knowledge. Learning and Instruction, 5(4), 261-267.

Bronfenbrenner, U. (1979). The Ecology of Human Development: Experiments by Nature and Design. Cambridge, MA: Harvard University Press.

Bronfenbrenner, U. (2001). The Bioecological Theory of Human Development. In N. J. Smelse \& P. B. Baltes (Eds.), International Encyclopaedia of the Social and Behavioural Sciences (Vol. 10, pp. 6963-6970). New York: Elsevier.

Bronfenbrenner, U. (2005a). Ecological Systems Theory. In U. Bronfenbrenner (Ed.), Making Human Beings Human: Bioecological Perspectives in Human Development (pp. 106-173). Thousand Oaks: Sage Publications.

Bronfenbrenner, U. (2005b). Making Human Beings Human: Bioecological Perspectives on Human Development. London: Sage.

Brosnan, K., \& Burgess, R. (2003). Web Based Continuing Professional Development: A Learning Architecture Approach. Journal of Workplace Learning, 15(1), 2333.

Brower, R. S., Abolafia, M., \& Carr, J. B. (2000). On Improving Qualitative Methods in Public Administration Research Administration and Society, 32(4), 363-397.

Brown, J., Collins, A., \& Duguid, P. (1989). Situated Cognition and the Culture of Learning. Educational Researcher, 18(1), 32-42.

Brown, J., \& Duguid, P. (1991). Organizational Learning and Communities of Practice: Toward a Unified View of Working, Learning and Innovation Organization Science, 2(1), 40-57.

Brown, J., \& Duguid, P. (1998). Organizing Knowledge. California Management Review 40(3), 90-111.

Brown, J., \& Duguid, P. (2001). Knowledge and Organization: A Social-Practice Perspective. Organization Science, 12(2), 198-213.

Brown, J. S., \& Duguid, P. (2000). The Social Life of Information. Boston: Harvard University Press.

Bruner, J. (1985). Narrative and Paradigmatic Modes of Thought. In E. Eisner (Ed.), Learning and Teaching the Ways of Knowing (84th Yearbook of the National Society for the Study of Education) (Vol. Part II, pp. 97-115). Chicago: University of Chicago Press.

Calderhead, J. (1988). The Development of Professional Knowledge Structures in Learning to Teach. In J. Calderhead (Ed.), Exploring Teachers' Thinking (pp. 51-64). London: Cassell. 
Carlile, P., \& Rebentisch, E. (2003). Into the Black Box: The Knowledge

Transformation Cycle. Management Science 49(9), 1180-1195.

Carter, K., \& Doyle, W. (1987). Teachers' Knowledge Structures and Comprehension Processes. In J. Calderhead (Ed.), Exploring Teachers' Thinking (pp. 147-160). London: Cassell.

Castro, M. (2004). The Community of Practice Ecosystem: On Competition, Cooperation, Differentiation, and the Role of Blogs. Knowledge Board. Retrieved from http://www.knowledgeboard.com/lib/1567

Castro, M. (2006). Revisiting Communities of Practice: From Fisherman Guilds to the Global Village. Paper presented at the 3rd European Knowledge Management Network Summer School, Madrid, Spain. Retrieved from http://www.knowledgeboard.com/item/2713.

Chai, K., Gregory, M., \& Shi, Y. (2003). Bridging Islands of Knowledge: A Framework of Knowledge Sharing Mechanisms. International Journal of Technology Management, 25(8).

Charmaz, K. (2006). Constructing Grounded Theory: A Practical Guide Through Qualitative Analysis. London: Sage.

Chin, R., \& Benne, K. (1969). General Strategies for Effecting Changes in Human Systems. In W. Bennis, K. Benne \& R. Chin (Eds.), The Planning Of Change (2nd ed., pp. 32-59). New York: Holt, Rinehart and Winston.

Clarke, H., \& Brennan, S. (1991). Grounding in Communication. In L. Resnik, J. Levine \& S. Teasley (Eds.), Socially Shared Cognition (pp. 17-149). Washington D.C.: American Psychology Association.

Cognition (2007). What Research is Saying. Online Presentation: Leadspace (Online Community for Principals, Ministry of Education, New Zealand).Retrieved 2 May, 2008 from www.leadspace.govt.nz/leadership/ict/ppt/what-research-issaying.ppt

Cohen, M., \& Bacdayan, P. (1994). Organizational Routines are Stored as Procedural Memory: Evidence from a Laboratory Study. Organization Science, 5(4), 554568.

Connelly, F., \& Clandinin, D. (1985). Personal Practical Knowledge and the Modes of Knowing: Relevance for Teaching and Learning. In E. Eisner (Ed.), Learning and Teaching the Ways of Knowing (84th Yearbook of the National Society for the Study of Education) (Vol. Part II, pp. 174-198). Chicago: University of Chicago Press.

Connelly, F., \& Clandinin, D. (1994 ). Telling Teaching Stories. Teacher Education Quarterly, 1, 145-158.

Cooper, A., Levin, B., \& Campbell, C. (2009). The Growing (but Still Limited) Importance of Evidence in Educational Policy and Practice. Journal of Educational Change, 10(2-3), 159-171.

Cornu, B. (2004). Networking and Collective Intelligence for Teachers and Learners. In A. Brown \& N. Davis (Eds.), World Yearbook of Education, 2004: Digital Technology, Communities and Education (pp. 251-259). London: RoutledgeFalmer.

Cox, A. (2005). What Are Communities of Practice? A Comparative Review of Four Seminal Works. Journal of Information Science, 31(6), 527-540.

Cranefield, J., \& Yoong, P. (2005). Organizational Factors Affecting Inter-organisational Knowledge Transfer in the New Zealand State Sector: A Case Study. The Electronic Journal for Virtual Organizations and Networks [online journal], (7), 933. Retrieved from http://www.ejov.org/

Cranefield, J., \& Yoong, P. (2006). The Stages of Inter-Organisational Knowledge Transfer: An Exploratory Study of the New Zealand State Sector International Journal of Knowledge, Culture and Change Management, 5(5), 75-84. 
Cranefield, J., \& Yoong, P. (2007a). Inter-Organisational Knowledge Transfer: The Role of the Gatekeeper. International Journal of Knowledge and Learning, 3(1), 121-138.

Cranefield, J., \& Yoong, P. (2007b). The Role of the Translator/ Interpreter in Knowledge Transfer Environments. Journal of Knowledge and Process Management, 14(2), 95-103.

Cranefield, J., \& Yoong, P. (2007c). To Whom Should Information Systems Research Be Relevant: The Case for an Ecological Perspective. In H. Österle, J. Schelp \& R. Winter (Eds.), Proceedings of the 15th European Conference on Information Systems (ECIS2007), June 7-9 2007, St. Gallen, Switzerland (pp. 1313-1324). St. Gallen: University of St. Gallen.

Cresswell, J. (1994). Research Design: Qualitative and Quantitative Approaches. Thousand Oaks, CA: Sage.

Cresswell, J. W. (2003). Research Design: Qualitative, Quantitative, and Mixed Methods Approaches (2nd ed.). Thousand Oaks, CA: Sage.

Cross, R., \& Prusak, L. (2002). The People Who Make Organizations Go - Or Stop. Harvard Business Review, 80(6), 104-112.

Crotty, M. (1998). The Foundations of Social Research: Meaning and Perspective in the Research Process. London: Sage.

Crowston, K., \& Myers, M. (2004). Information Technology and the Transformation of Industries: Three Research Perspectives. The Journal of Strategic Information Systems, 13(1), 5-28.

Darke, P., Shanks, G., \& Broadbent, M. (1998). Successfully Completing Case Study Research: Combining Rigour, Relevance and Pragmatism. Information Systems Journal, 8(4), 273-289.

Davenport, E. (2004). Double Agents: Visible and Invisible Work in an Online Community of Practice. In P. Hildreth \& C. Kimble (Eds.), Knowledge Networks: Innovation through Communities of Practice (pp. 256-266): Idea Group Publishing.

Davenport, T. (1997). Information Ecology: Mastering the Information and Knowledge Environment. New York: Oxford University Press.

Davenport, T., \& Prusak, L. (1998). Working Knowledge: How Organizations Manage what they Know. Boston: Harvard Business School Press.

Davidson, C., \& Voss, P. (2002). Knowledge Management: An Introduction to Creating Competitive Advantage from Intellectual Capital. Auckland: Tandem Press.

Denzin, N., \& Lincoln, Y. (2000). Handbook of Qualitative Research (2nd ed.). California: Sage.

Denzin, N., \& Lincoln, Y. (2005). The Sage Handbook of Qualitative Research. Thousand Oaks, CA: Sage.

Dewey, J. (1933). How We Think. A Restatement of the Relation of Reflective Thinking to the Educative Process (Revised ed.). Boston: D.C.: Heath.

Dodge, B. (1995). WebQuests: A Technique for Internet-based Learning. Distance Educator, 1(2), 10-13.

Drazin, R., Glynn, M. A., \& Kazanjiian, R. K. (1999). Multilevel Theorizing about Creativity in Organizations: A Sensemaking Perspective. Academy of Management Review, 24(2), 286-307.

Dubé, L., Bourhis, A., \& Jacob, R. (2006). Towards a Typology of Virtual Communities of Practice. Interdisciplinary Journal of Information, Knowledge, and Management, 1, 69-93.

Dubé, L., \& Paré, G. (2003). Rigor in Information Systems Positivist Case Research: Current Practices, Trends, and Recommendations. MIS Quarterly, 27(4), 597636.

Dunford, R. (2000). Key Challenges in the Search for Effective Management of Knowledge in Management Consulting Firms. Journal of Knowledge Management, 4(4), 295-302. 
Dunne, R., \& Wragg, T. (1994). Effective Teaching. London: Routledge.

Earl, L., Katz, S., Elgie, S., Ben Jaafar, S., \& Foster, L. (2006). How Networked Learning Communities Work: Aporia Consulting Ltd. Retrieved from http://networkedlearning.ncsl.org.uk/collections/network-researchseries/summaries/how-networked-learning-communities-work-summary.pdf.

Earl, M., \& Scott, I. (1998). What on Earth is a CKO? Survey IBM: London.

Edwards, M. (2005). The Integral Holon: A Holonomic Approach to Organisational Change and Transformation. Journal of Organizational Change Management 18(3), 269-288.

Efimova, L., \& Hendrik, S. (2005). In Search For a Virtual Settlement: An Exploration of Weblog Community Boundaries Updated version of a paper presented at Communities and Technologies Conference 2005. Retrieved 10 May, 2008 from https://doc.telin.nl/dsweb/Get/Document46041/weblog_community_boundaries.pdf.

Eisenhardt, K. (1989). Building Theories from Case Study Research. Academy of Management Review, 14(4), 532-550.

Elbaz, F. (1983). Teacher Thinking: A Study of Practical Knowledge. London: Falmer Press.

Engeström, Y., Engeström, R., \& Kärkkäinen, M. (1995). Polycontextuality and Boundary Crossing in Expert Cognition: Learning and Problem Solving in Complex Work Activities. Learning and Instruction, 5(4), 319-336.

English, M., \& Baker, W. (2006). Winning the Knowledge Transfer Race. New York: McGraw-Hill.

Farkas, M., Jette, A., Tennstedt, S., Haley, S., \& Quinn, V. (2003). Knowledge Dissemination and Utilization in Gerontology: An Organizing Framework. The Gerontologist, 43(Special Issue 1), 47-56.

Fitzgerald, B., \& Howcroft, D. (1998). Towards Dissolution of the IS Research Debate: From Polarisation to Polarity. Journal of Information Technology, 13(4), 313326.

Fontaine, M. (2001). Keeping Communities of Practice Afloat: Understanding and Fostering Roles in Communities. Knowledge Management Review, 4(4), 16-21.

Fontaine, M., \& Millen, D. (2004). Understanding the Benefits and Impact of Communities of Practice. In P. Hildreth \& C. Kimble (Eds.), Knowledge Networks: Innovation through Communities of Practice (pp. 1-13). Hershey, PA: Idea Group Publishing.

Fox, R., Lidz, V., \& Bershady, H. (2005). Introduction In R. Fox, V. Lidz \& H. Bershady (Eds.), After Parsons: A Theory of Social Action for the Twenty-First Century (pp. 1-30). New York: Russell Sage Foundation.

Friedman, M., \& Farag, Z. E. (1991). Gaps in the Dissemination/Knowledge Utilization Base. Science Communication, 12(3), Knowledge: Creation, Diffusion, Utilization, 266-288.

Fullan, M. (2009). Large-scale Reform Comes of Age. Journal of Educational Change 10(2-3), 101-113.

Gawith, G. (2004). Less is More. Good Teacher Magazine (Term 1), 1-2.

Giddens, A. (1987). Social Theory and Modern Sociology. Cambridge: Polity Press.

Gilbert, D. (1999). What the Mind's Not. In S. Chaiken \& Y. Trop (Eds.), Dual Process Theories in Social Psychology (pp. 3-11). New York: Guilford Press.

Gilbert, J. (2005). Catching the Knowledge Wave? The Knowledge Society and the Future of Education. Wellington, NZ: NZCER Press.

Gladwell, M. (2000). The Tipping Point: How Little Things Can Make a Big Difference London: Little Brown.

Golafshani, N. (2003). Understanding Reliability and Validity in Qualitative Research. The Qualitative Report, 8(4), 597-607.

Gongla, P., \& Rizzuto, C. (2001). Evolving Communities of Practice: IBM Global Services Experience. IBM Systems Journal, 40(4), 842-863. 
Goodfellow, R. (2003). Virtual Learning Communities: A Report for the National College for School Leadership (NCSL): The Institute of Educational Technology, The Open University, England. Retrieved from http://kn.open.ac.uk/public/document.cfm?docid=2778

Goodman, R., \& Steckler, A. (1989). A Model for the Institutionalisation of Health Promotion Programs. Family and Community Health, 11(4), 63-78.

Goodwin, C. (1990). Perception, Technology and Interaction on a Scientific Research Vessel. Paper presented at the 89th Annual Meeting of the American Anthropological Association. (Cited by Engeström et al., 1995, p.320)

Gorgoglione, M. (2003). Strategies for Knowledge Transfer Knowledge Board (Global Community for Knowledge Management. Retrieved 24 October, 2005 from http://www.knowledgeboard.com/cgibin/item.cgi?id=110132\&d=1\&h=417\&f=418\&dateformat=\%0\%20\%B\%20\%Y.

Gorman, G., \& Clayton, P. (2005). Qualitative Research for the Information Professional (2nd ed.). London: Facet.

Gray, B. (2004). Informal Learning in an Online Community of Practice. Journal of Distance Education, 19(1), 20-35.

Greenhalgh, T., Robert, G., MacFarlane, F., Bate, P., \& Kyriakidou, O. (2004). Diffusion of Innovations in Service Organisations: Systematic Review and Recommendations. The Milbank Quarterly, 82(4), 577-786.

Greenhalgh, T., Robert, G., Macfarlane, F., Bate, P., Kyriakidou, O., \& Peacock, R. (2005). Storylines of Research in Diffusion of Innovation: A Meta-Narrative Approach to Systematic Review. Social Science and Medicine, 61, 417-430.

Gregor, S. (2006). The Nature of Theory in Information Systems. MIS Quarterly, 30(3), 611-642.

Grossman, P. (1995). Teachers' Knowledge. In L. Anderson (Ed.), International Encyclopedia of Teaching and Teacher Education (2nd ed., pp. 20-24). Kidlington, Oxford, UK: Elsevier Science Ltd.

Ham, V. (2005). Clustering Schools for Teacher Development in ICT: The 'Grand Experiment'. In K. Lai (Ed.), E-Learning Communities: Teaching and Learning with the Web. Dunedin: Otago University Press.

Ham, V. (2008). National Trends in the ICT PD School Clusters Programme 20042006. Wellington, New Zealand: Ministry of Education. Retrieved from http://www.educationcounts.govt.nz/publications/ict/5819/22930.

Handal, B. (2004). Teachers' Instructional Beliefs about Integrating Educational Technology. E-Journal of Instructional Science and Technology, 17(1), 1-10.

Handal, B., \& Herrington, A. (2003). Mathematics Teachers' Beliefs and Curriculum Reform Mathematics Education Research Journal, 15(1), 59-69.

Hara, N., \& Kling, R. (2002). Communities of Practice with and without Information Technology. In E. Rasmussen \& E. Toms (Eds.), Proceedings of the 65th Annual Meeting of the American Society for Information Science and Technology (Vol. 39, pp. 338-349). Medford, NJ: Information Today, Inc.

Harada, T. (2003). Three Steps in Knowledge Communication: The Emergence of Knowledge Transformers. Research Policy, 32(10), 1737-1751.

Harasim, L. (1990). Online Education: Perspectives on a New Environment. New York: Praeger.

Hargreaves, D. (2003). Education Epidemic: Transforming Secondary Schools through Innovation Networks. Retrieved from http://www.demos.co.uk/media/educationepidemic page277.aspx

Harragon, A., \& Sutton, R. (1997). Technology Brokering and Innovation in a Product Development Firm Administrative Science Quarterly, 42, 716-749.

Hemmasi, M., \& Csanda, C. (2009). The Effectiveness of Communities of Practice: An Empirical Study. Journal of Managerial Issues, 21(2), 262-279.

Hernandez, H., Liang, Q., Prescott, P., \& Kirch, A. (2004). Gotcha! Guide to Knowledge Management (UC Berkeley's School of Information Management \& Systems) 
Retrieved from

http://sims.berkeley.edu/courses/is213/s99/Projects/P9/web site/glossary.htm

Hew, K., \& Hara, N. (2007a). Empirical Study of Motivators and Barriers of Teacher Online Knowledge Sharing. Educational Technology, Research and

Development, 55(6), 573-595.

Hew, K., \& Hara, N. (2007b). Knowledge Sharing in Online Environments: A Qualitative Case Study. Journal of the Association for Information Science and Technology, 58(14), 2310-2324.

Hildreth, P., Wright, P., \& Kimble, C. (1998). Computer Mediated Communications and International Communities of Practice Proceedings of Ethicomp '98 (pp. 275286). Erasmus University, The Netherlands. Available at http://www.chriskimble.com/Publications/Documents/Hildreth 1998.pdf.

Hippel, E. (1991). 'Sticky Information' and the Locus of Problem Solving. Management Science, 44, 477-490.

Hodkinson, P. (2006). Subcultural Blogging: Online Journals and Group Involvement Among UK Goths', in Bruns, A. and Jacobs, Y. (eds.), Uses of Blogs, Peter Lang 2006. . In A. Bruns \& Y. Jacobs (Eds.), Uses of Blogs, Peter Lang 2006. (pp. 187-198). New York: Peter Lang

Holden, N., \& Von Kortzfleisch, H. (2004). Why Cross-Cultural Knowledge Transfer is a Form of Translation in More Ways than You Think. Knowledge and Process Management, 11(2), 127-137.

Huberman, A. M., \& Miles, M. B. (1998). Data Management and Analysis Methods. In N. K. Denzin \& Y. S. Lincoln (Eds.), Collecting and Interpreting Qualitative Materials (pp. 179-210). London: Sage.

Hutchinson, J., \& Huberman, M. (1993). Knowledge Dissemination and Use in Science and Mathematics Education: A Literature Review. National Science Foundation.

Johnson, C. (2001). A Survey of Current Research on Online Communities of Practice. The Internet and Higher Education(4), 45-60.

Johnson, M., Kazakov, D., \& Svehla, M. (2005). ICT in Schools Report: Information and Communications Technology in New Zealand Schools 1993-2005. Wellington, New Zealand: 2020 Communications Trust.

Kagan, D. (1990). Ways of Evaluating Teacher Cognitions: Inferences Concerning the Goldilocks Principle. Review of Educational Research, 60, 419-470.

Kaiser, S., Müller-Seitz, G., Pereira Lopes, M., \& Pina e Cunha, M. (2007). WeblogTechnology as a Trigger to Elicit Passion for Knowledge. Organization, 14(3), 391-412.

Kaplan, B., \& Maxwell, J. A. (1994). Qualitative Research Methods for Evaluating Computer Information Systems. In J. Anderson, C. Aydin \& S. Jay (Eds.), Evaluating Health Case Information Systems: Methods and Applications (pp. 45-68). California: Sage.

Kaplan, S. (2002). Building Communities - Strategies for Collaborative Learning. In Learning Circuits: American Society for Training \& Development. Retrieved 20 May, 2009 from http://www.astd.org/LC/2002/0802 kaplan.htm.

Katz, R., \& Tushman, M. (1981). An Investigation into the Managerial Roles and Career Paths of Gatekeepers and Project Supervisors in a Major R\&D Facility. $R$ \& D Management, 11, 103-110.

Kayes, A. B., Kayes, D. C., \& Yamazaki, Y. (2005). Transferring Knowledge Across Cultures: A Learning Competencies Approach. Performance Improvement Quarterly, 18(4), 87-100.

Kew, K. F., \& Hara, N. (2007). Empirical Study of Motivators and Barriers of Teacher Online Knowledge Sharing. Educational Technology, Research and Development, 55(6), 573-595.

Keys, P., M. (2006). A Knowledge Filter Model for Observing and Facilitating Change in Teachers' Beliefs. Journal of Educational Change, 8(1), 41-60. 
Klein, H., \& Myers, M. (1999). A Set of Principles for Conducting and Evaluating Interpretive Field Studies in Information Systems. MIS Quarterly, 23(1), 67-93.

Klein, K., Tosi, H., \& Cannella, A. (1999). Multilevel Theory Building: Benefits, Barriers and New Developments (Introduction to Special Topic Forum) The Academy of Managment Review, 24(2), 243-248.

Kogut, B., \& Zander, U. (1992). Knowledge of the Firm, Combinative Capabilities, and the Replication of Technology. Organization Science, 3, 383-397.

Krefting, L. (1991). Rigor in Qualitative Research: The Assessment of Trustworthiness. The American Journal of Occupational Therapy, 45(3), 214-222.

Kwan, M., \& Cheung, P. (2006). The Knowledge Transfer Process: From Field Studies to Technology Development. Journal of Database Management, 17(1), 16-32.

Lai, K. (2005). E-Learning Communities: Teaching and Learning with the Web. Dunedin: University of Otago Press.

Lai, K., Pratt, K., Anderson, M., \& Stigter, J. (2005). Literature Synthesis and Review: Online Communities of Practice. Retrieved 6 July, 2006 from http://educationcounts.edcentre.govt.nz/publications/curriculum//rs-onlinecom.html.

Lave, J., \& Wenger, E. (1991). Situated Learning: Legitimate Peripheral Participation. Cambridge, UK: Cambridge University Press.

Lee, M., \& Lan, Y. (2007). From Web 2.0 to Conversational Knowledge Management: Towards Collaborative Intelligence Journal of Entrepreneurship Research 2(2), 47-62.

Leinhardt, G. (1988). Situated Knowledge and Expertise in Teaching. In J. Calderhead (Ed.), Teachers' Professional Learning (pp. 146-168). London: Falmer Press.

Leinhardt, G., Young, K. M., \& Merriman, J. (1995). Integrating Professional Knowledge: The Theory of Practice and the Practice of Theory. Learning and Instruction, 5(4), 401-408.

Lerner, R. (2002). Concepts and Theories of Human Development (3rd ed.). Mahwah, New Jersey: Lawrence Erlbaum.

Lerner, R. (2005). Urie Bronfenbrenner: Career Contributions of the Consumate Developmental Scientist. In U. Bronfenbrenner (Ed.), Making Human Beings Human: Bioecological Pespectives on Human Development (pp. ix-xxvi (Forward)). Thousand Oaks: Sage Publications.

Lesser, E., \& Storck, J. (2001). Communities of Practice and Organizational Performance. IBM Systems Journal, 40(4), 831-841.

Levina, N., \& Vaast, E. (2005). The Emergence of Boundary Spanning Competence in Practice: Implications for Implementation and Use of Information Systems MIS Quarterly, 29(2), 335-363.

Lewin, K. (1952). Field Theory in Social Science. London: Tavistock.

Lewthwaite, B. (2006). "I want to enable teachers in their change": Exploring the Role of a Superintendent on Science Curriculum Delivery Canadian Journal of Educational Administration and Policy [online](52), 1-23. Retrieved fom http://www.umanitoba.ca/publications/cjeap/articles/lewthwaite.html.

Lincoln, Y., \& Guba, E. (1985). Naturalistic Inquiry. Beverly Hills, CA: Sage.

Louden, W. (1991). Understanding Teaching: Continuity and Change in Teachers' Knowledge New York: Teachers College Press.

Madhaven, R., \& Grover, R. (1998). From Embedded Knowledge to Embodied Knowledge: New Product Development as Knowledge Management. Journal of Marketing, 62(4), 1-12.

Major, E., \& Cordey-Hayes, M. (2001). Foresight as a Core Competence. Futures, 33(2), 91-108.

MaKinster, J. G., Barab, S. A., Harwood, W. S., \& Anderson, H. O. (2006). The Effect of Social Context on the Reflective Practice of Pre-Service Science Teachers: Incorporating a Web-Supported Community of Teachers. Journal of Technology and Teacher Education, 14(3), 543-579. 
Malhotra, Y. (2002). Information Ecology and Knowledge Management: Toward Knowledge Ecology for Hyperturbulent Organizational Environments Encyclopaedia of Life Support Systems (EOLSS) Oxford: UNESCO/Eolss Publishers.

Martin, P. (1993). An Evaluation of the Effects of the Victorian Certificate of Education on Mathematics Teachers. Paper presented at the 16th Annual Conference of the Mathematics Education Research Group (MERGA), Brisbane. Cited by Handal and Herrington, 2003.

McCormick, R. (2002). Theroetical Perspectives of Relevance to Networked Learning Communities: Centre for Curriculum and Teaching Studies, The Open University. Retrieved from http://networkedlearning.ncsl.org.uk/knowledgebase/research-papers/theoretical-perspectives-of-relevance-to-nlcs.pdf

McDermott, R. (2000). Community Development as a Natural Step. Knowledge Management Review, 3(5), 16-19.

McGrath, J. E., \& Argote, L. (2001). Group Processes in Organizational Contexts. . In M. A. In Hogg \& R. S. Tindale (Eds.), Blackwell Handbook of Social Psychology: Group Processes (Vol. 3, pp. 603-627). Oxford: Blackwell.

McGregor, J., Fielding, M., Robinson, C., \& Spender, B. (2006). Footprints of Practice: Exploring the Sharing and Development of Practice Through Collaborative Adult Learning: National College for School Leadership (NCSL).

McLeroy, K., Bibeau, D., \& Huberman, A. (1988). An Ecological Perspective on Health Promotion Programs. Health Education Quarterly, 15(4), 351-377.

Miles, M., \& Huberman, A. (1984). Qualitative Data Analysis: A Sourcebook of New Methods. California: Sage.

Miles, M., \& Huberman, A. M. (1994). Qualitative Data Analysis: An Expanded Sourcebook (2nd ed.). Thousand Oaks, CA: Sage.

Minister for Information Technology's IT Advisory Group (1999). The Knowledge Economy

Ministry of Education (2003). Effective Literacy Practice in Years 1 to 4. Wellington, New Zealand: Learning Media

Ministry of Education (2004). Statement of Intent, 2004-2009. Wellington, New Zealand: Author.

Ministry of Education (2005a). Briefing for the Incoming Minister of Education, October 2005. Wellington, New Zealand: Author. Retrieved 21 October, 2006 from http://www.minedu.govt.nz/index.cfm?layout=document\&documentid=10907.

Ministry of Education (2005b). Report of the Findings of the 2004 Teacher Census. Wellington, New Zealand: Author.

Ministry of Education (2006a). Enabling the 21st Century Learner - An E-Learning Action Plan for Schools 2006-2010. Wellington, New Zealand: Author. Retrieved from http://www.minedu.govt.nz/index.cfm?layout=document\&documentid=10475\&in dexid=1024\&indexparentid $=1072$.

Ministry of Education (2006b). Making a Good Education System Great (Speech by Howard Fancy, Secretary for Education. 18th May, 2006). Retrieved May 24, 2006. from http://mediacentre.minedu.govt.nz/speeches/2006/2006 009 1805.html.

Ministry of Education (2006c). Statement of Intent, 2006-2010. Wellington, New Zealand: Author.

Ministry of Education (2007a). The New Zealand Curriculum. Wellington Learning Media.

Ministry of Education (2007b). The New Zealand Curriculum Online. Retrieved 7 November 2008, from http://nzcurriculum.tki.org.nz/About-this-site/Frequentlyasked-questions-FAQ 
Ministry of Education (2008). Education Sectors Website. Retrieved 2 May, 2009. from http://www.minedu.govt.nz/educationSectors/Schools/Initiatives/ICTInSchools/l CTStrategy/LatestICTNewsAndReleases/ELearningTeacherFellowships.aspx.

Mishler, E. G. (2000). Validation in Inquiry-Guided Research: The Role of Exemplars in Narrative Studies. In B. M. Brizuela, J. P. Stewart, R. G. Carrillo \& J. G. Berger (Eds.), Acts of Enquiry in Qualitative Research (pp. 119-146). Cambridge, MA: Harvard Educational Review.

Munby, H., Russell, T., \& Martin, A. (2001). Teachers' Knowledge and How It Develops. In V. Richardson (Ed.), Handbook of Research on Teaching (Fourth ed., pp. 877-904). Washington, DC: American Educational Research Association.

Murillo, E. (2008). Searching Usenet for Virtual Communities of Practice: Using Mixed Methods to Identify the Constructs of Wenger'sTheory. Information Research, 13(4), paper 386. Retrieved from http://informationr.net/ir/13-4/paper386.html

Myers, M. (1997). Qualitative Research in Information Systems. MIS Quarterly, 21(2), 241-242.

Nelson, R., \& Winter, S. (1982). An Evolutionary Theory of Economic Change. Cambridge, CA: Belknap.

Nichani, M., \& Hung, D. (2002). Can a Community of Practice Exist Online? Educational Technology, 42(2), 49-54.

Nonaka, I. (1998). The Knowledge-Creating Company Harvard Business Review on Knowledge Management Boston: Harvard Business School Publishing.

Nonaka, I., \& Konno, N. (1998). The Concept of "Ba": Building a Foundation for Knowledge Creation. California Management Review, 40(3), 40-54.

Nonaka, I., \& Takaeuchi, N. (1995). The Knowledge Creating Company: How Japanese Companies Create the Dynamics of Innovation. New York: Oxford University Press.

Nonnecke, B., Andrews, D., \& Preece, J. (2006). Non-public and Public Online Community Participation: Needs, Attitudes and Behaviour. Electronic Commerce Research, 6, 7-20.

Nonnecke, B., \& Preece, J. (1999). Shedding Light on Lurkers in Online Communities. In K. Buckner (Ed.), Proceedings of Ethnographic Studies in Real and Virtual Environments: Inhabited Information Spaces and Connected Communities (pp. 123-128). Edinburgh.

Nonnecke, B., Preece, J., Andrews, D., \& Voutour, R. (2004). Online Lurkers Tell Why. AMCIS 2004 Proceedings. Paper 321. Available at http://aisel.aisnet.org/amcis2004/321.

Nye, B., Konstantopolous, S., \& Hedges, L. (2004). How Large Are Teacher Effects? Educational Evaluation and Policy Analysis, 26(3), 237-257.

Organisation for Economic Co-operation and Development (OECD) (1999). Managing National Innovation Systems. Paris: OECD.

Organisation for Economic Co-operation and Development (OECD) (2000). The Programme for International Student Assessment (PISA). Paris: OECD.

Organisation for Economic Co-operation and Development (OECD) (2003). New Challenges for Educational Research. Paris: OECD.

Organisation for Economic Co-operation and Development (OECD) (2005). School Factors Related to Quality and Equity: Results from PISA 2000. Paris: OECD.

Orlikowski, W. J. (2002). Knowing in Practice: Enacting a Collective Capability in Distributed Organizing. Organization Science, 13(3), 249-274.

Orlikowski, W. J., \& Baroudi, J. (1991). Studying Information Technology in Organizations: Research Approaches and Assumptions. Information Systems Research, 2(1), 1-28.

Parsons, T. (1937). The Structure of Social Action. A Study in Social Theory with Special Reference to a Group of Recent European Writers (2nd ed.). New York: MacGraw Hill. 
Parsons, T. (1949). The Structure of Social Action. A Study in Social Theory with Special Reference to a Group of Recent European Writers. (2nd ed.). Glencoe, IL: Free Fress.

Parsons, T. (1951). The Social System. London: Routledge and Kegan Paul Ltd.

Patton, M. Q. (1990). Qualitative Evaluation and Research Methods (2nd ed.). Newbury Park, CA: Sage.

Pauleen, D. (2009). Personal Knowledge Management: Putting "Person" back into the Equation. Online Information Review, 33 (2), 221-224.

Pawlowski, S., \& Robey, D. (2004). Bridging User Organizations: Knowledge Brokering and the Work of Information Technology Professionals. MIS Quarterly, 28(4), 645-672.

Pawlowski, S., Robey, D., \& Raven, A. (2000). Supporting Shared Information Systems: Boundary Objects, Communities, and Brokering. ICIS 2000 Proceedings. Paper 30. Available at http://aisel.aisnet.org/icis2000/30

Polanyi, M. (1958). Personal Knowledge: Towards a Post-Critical Philosophy. London: Routledge and Kegan Paul.

Polanyi, M. (1967). The Tacit Dimension. Chicago: University of Chicago Press.

Prensky, M. (2001). Digital Natives, Digital Immigrants. From On the Horizon (NCB University Press), 9(5), 1-2.

Prensky, M. (2005-6). Listen to the Natives. Educational Leadership, 63: Learning in the Digital Age(4), 8-13.

Rafaeli, S., Ravid, G., \& Soroka, V. (2004). De-lurking in Virtual Communities: A Social Communication Network Approach to Measuring the Effects of Social and Cultural Capital. Proceedings of the 37th Annual Hawaii International Conference on System Sciences (HICSS'04), vol 7,pp. 70203. Available at http://doi.ieeecomputersociety.org/10.1109/HICSS.2004.1265478.

Reder, S. (1993). Watching Flowers Grow: Polycontextuality and Heterochronicity at Work. The Quarterly Newsletter of Comparative Human Cognition, 15(4), 116125 (Cited by Engeström et al., 1995, p.1320)

Reed, R., \& DeFillippi, R. (1990). Causal Ambiguity, Barriers to Imitation, and Sustainable Competitive Advantage. Academy of Management Review, 15(1), 88-102.

Reynolds, S., Anderson, J., \& Calvo, A. (1997). Ecological Models of Health Behaviour and Health Promotion. University of South Florida Health and Community Website. Retrieved 27 July, 2006, from http://hsc.usf.edu/ kmbrown/Ecological Models Overview.htm

Rich, R. (1991). Knowledge Creation, Diffusion, and Utilization. Science Communication, 12(3), 319-337.

Richardson, V., \& Placier, P. (2001). Teacher Change. In V. Richardson (Ed.), Handbook of Research on Teaching (4th ed., pp. 905-947). Washington, DC. : American Educational Research Association.

Robinson, S. K. (2005). Do Schools Kill Creativity? Paper presented at the National Forum on Education Policy, Education Commission of the United States, Chairman's Breakfast Retrieved May 4 2007, from http://www.ecs.org/html/projectsPartners/chair2005/docs/Sir Ken Robinson S peech.pdf.

Rogers, E. (1962). The Diffusion of Innovations. New York: The Free Press.

Rogers, E. (1983). The Diffusion of Innovations (3rd ed.). New York: The Free Press.

Rogers, E. (2003). The Diffusion of Innovations (5th ed.). New York: The Free Press.

Rolland, N., Chauvel, D., \& Despres, C. (2003). Knowledge Transfer and Organisational Learning in Strategic Alliances. In A. Bono (Ed.), Research in Knowledge Management Consulting (Vol. 3: Enhancing Inter-firm Networks and Inter-organizational Strategies, pp. 93-116).

Rovai, A. (2002). Building Sense of Community at a Distance. International Review of Research in Open and Distance Learning, 3(1), 1-12. 
Rowlands, B. (2005). Grounded in Practice: Using Interpretive Research to Build Theory. The Electronic Journal of Business Research Methodology 3(1), 81-92. Available at www.ejbrm.com.

Rowling, J. (1997). Harry Potter and the Philosopher's Stone. London: Bloomsbury.

Saint-Onge, H., \& Wallace, D. (2003). Leveraging Communities of Practice for Strategic Advantage. Boston, MA: Butterworth-Heinemann.

Sanchez, R. (2005). Knowledge Management and Organizational Learning: Fundamental Concepts for Theory and Practice. . Working Paper Series, Lund University, Institute of Economic Research (3). Retrieved from http://econpapers.repec.org/paper/hhblufewp/2005 5F003.htm

Sarker, S. (2007). Qualitative Research Genres in the IS Literature: Emerging Issues and Potential Limitations. Proceedings of the 40th Annual Hawaii International Conference on System Sciences (HICSS'07), 244-253. Available at http://doi.ieeecomputersociety.org/10.1109/HICSS.2007.456

Sarker, S., Sarker, S., Nicholson, D. B., \& Joshi, K. (2005). Knowledge Transfer in Virtual Systems Development Teams: An Exploratory Study of Four Key Enablers. IEEE Transactions on Professional Communication, 48(2), 201-218.

Scharmer, C. O. (2001). Self-Transcending Knowledge: Organizing around Emerging Realities. In D. In Teece (Ed.), Managing Industrial Knowledge: Creation, Transfer, and Utilization (pp. 68-90). Thousand Oaks, CA: Sage.

Schlager, M., \& Fusco, J. (2003). Teacher Professional Development, Technology, and Communities of Practice: Are We Putting the Cart Before the Horse? The Information Society, 19, 203-220.

Schmitz-Justen, F., \& Wilhelm, A. (2005, 14-16 December ). Factors Influencing and Supporting Key Knowledge Processes in Online Knowledge Communities: Results of a SEM-based Exploraory Case Study. Paper presented at the IADIS International Conference on Cognition and Exploratory Learning in Digital Age (CELDA 2005), Porto, Portugal.

Schön, D. (1983). The Reflective Pracititioner. How Professionals Think in Action New York: Basic Books.

Seale, C. (1999). The Quality of Qualitative Research. London: Sage.

Sharatt, M., \& Usoro, A. (2003). Understanding Knowledge-Sharing in Online Communities of Practice. Electronic Journal of Knowledge Management 1(2). Retrieved from www.ejkm.com/volume-1/volume1-issue.../issue2-art18sharratt.pdf

Smith, S. Z., Smith, M. E., \& Williams, S. (2005). Elaborating a Change Process Model for Elementary Mathematics Teachers' Beliefs and Practices Current Issues in Education [online journal], 8 (19). Available at http://cie.ed.asu.edu/volume8/number19/.

Snyder, W., \& Wenger, E. (1999). Communities of Practice: Lessons Learned from Auburn Hills. Stuttgart: Daimler-Chrysler Corporate University.

Soroka, V., \& Rafaeli, S. (2006). Invisible Participants: How Cultural Capital Relates to Lurking Behaviour Proceedings of the 2006 International Conference on the World Wide Web 2006. pp. 163-172. Available at http://doi.acm.org/10.1145/1135777.1135806.

Spender, J., \& Grant, R. (1996). Knowledge and The Firm: Overview. Strategic Management Journal, 17(Winter Special Issue), 5-9.

Star, S. (1989). The Structure of III-Structured Solutions: Boundary Objects and Heterogeneous Distributed Problem Solving. In L. Gasser \& M. Huhns (Eds.), Distributed Artificial Intelligence (Vol. II, pp. 37-54). London: Pitman (Cited by Engeström et al. 1995, p. 322).

Statistics New Zealand (2008). Human Capital Statistics Part 3: The Providers of Education and Training, and the Learning Environment. Department of Statistics Website Retrieved 10th January 2009, from 
http://www.stats.govt.nz/analytical-reports/human-capital-statistics/part-3providers-of-education-and-training.htm

Szulanski, G. (1996). Exploring Internal Stickiness: Impediments to the Transfer of Best Practice within the Firm. Strategic Management Journal, 17(Winter Special Issue), 27-43.

Szulanski, G. (2000). The Process of Knowledge Transfer: A Diachronic Analysis of Stickiness. Organizational Behaviour and Human Decision Processes, 82(1), 927.

Tarmizi, H., \& De Vreede, G. (2005). A Facilitation Task Taxonomy for Communities of Practice. AMCIS 2005 Proceedings. Paper 485. Available at http://aisel.aisnet.org/amcis2005/485.

Teigland, R., \& Wakso, M. (2004). Extending Richness with Reach: Participation and Knowledge Exchange in Electronic Networks of Practice. In P. Hildreth \& C. Kimble (Eds.), Knowledge Networks: Innovation through Communities of Practice (pp. 230-242). Hershey, PA: Idea Group Publishing.

Thomson, R., Reeves-Lipscombe, D., Stuckey, B., \& Mentis, M. (2005). Discourse Analysis and Role Adoption in a Community of Practice. Retrieved from http://www.cpsquare.org/stuckey-etal-AERA-Discourse analysis.pdf

Thorpe, M. (2003a). A Report on the Concept of Communities of Practice and its Applicability to NCSL's Networked Communities: Report 1 for NCSL Networked Communities: The Institute of Educational Technology, The Open University, England. Retrieved from http://networkedlearning.ncsl.org.uk/knowledgebase/research-papers/concept-of-communities-of-practice-report1-thorpe2003.doc.

Thorpe, M. (2003b). A Report on the Conceptualisation of Knowledge and Learning in Studies of Practice in Organizational and Professional Contexts: Report 3 on Communities of Practice, for NCSL Networked Communities: The Institute of Educational Technology, The Open University, England. Retrieved from http://networkedlearning.ncsl.org.uk/knowledge-base/researchpapers/conceptualisation-of-knowledge-and-learning-report3-thorpe-2003.doc.

Thorpe, M., \& Jelfs, A. (2004). Applications of a Communities of Practice Approach in Organisations: Report 4 on Communities of Practice, for NCSL Networked Communities: The Institute of Educational Technology, The Open University, England. Retrieved from http://networkedlearning.ncsl.org.uk/knowledgebase/research-papers/applications-of-comunities-of-practice-report4-thorpe2004.doc.

Tillema, H. H. (1995). Changing the Professional Knowledge and Beliefs of Teachers: A Training Study. Learning and Instruction, 5(4), 291-318.

Timbrell, G., Lambe, P., \& Taule, O. (2007). Throwing Pebbles into a Dark Cave:A Study of Participation and Behaviour in the \{act-km\} Online Community. Journal of Information and Knowledge Management, 6(2), 139-152.

Tsoukas, H. (1996). The Firm as a Distributed Knowledge System: A Constructionist Approach. Strategic Management Journal, 17, 11-25.

Tuggle, F., \& Goldfinger, W. (2004). A Methodology for Mining Embedded Knowledge from Process Maps. Human Systems Management, 23(1), 1-13.

Tushman, M. L. (1977). Special Boundary Roles in the Innovation Process. Administrative Science Quarterly, 22(4), 587-605.

Van Buren, M. (1999). A Yardstick for Knowledge Management. Training and Development Journal, 53(5), 71-78.

Van de Ven, A. H., \& Poole, M. S. (2000). A Typology of Process Theories. In M. S. Poole, A. Van de Ven, K. Dooley \& M. Holmes (Eds.), Organizational Change and Innovation Processes (pp. 57-88). Oxford: Oxford University Press.

Van Maanen, J. (1989). Some Notes on the Importance of Writing in Organizational Studies. In J. Cash \& P. Lawrence (Eds.), The Information Systems Research Challenge: Volume 1 (pp. 27-33). Boston: Harvard Business School. 
Wagenaar, W. (1986). My Memory: A Study of Autobiography Memory Over Six Years. Cognitive Psychology 18, 225-252.

Wagner, C., \& Bolloju, N. (2005). Supporting Knowledge Management in Organizations with Conversational Technologies: Discussion Forums, Weblogs, and Wikis. Journal of Database Management, 16(2), 1-8.

Walsh, J., \& Ungston, G. (1991). Organizational Memory. Academy of Management Review, 16(1), 57-91.

Walsham, G. (1995). Interpretive Case Studies in IS Research: Nature and Method. European Journal of Information Systems, 4, 74-81.

Wasko, M., \& Faraj, S. (2005). Why Should I Share? Examining Social Capital and Knowledge Contribution in Electronic Networks of Practice. MIS Quarterly, 29(1), 35-57.

Wasko, M., \& Teigland, R. (2002). The Provision of Online Public Goods: Examining Social Structure. ICIS 2002 Proceedings. Paper 15. Available at http://aisel.aisnet.org/icis2002/15.

Wasko, M., \& Teigland, R. (2004). Public Goods or Virtual Commons? Applying Theories of Public Goods, Social Dilemmas and Collective Action to Electronic Networks of Practice. Journal of Information Technology Theory and Applications, 6(1), 25-42.

Wei, C. (2004). Formation of Norms in a Blog Community. Into the Blogosphere; Rhetoric, Community and Culture of Weblogs. In S. A. L.Gurak, L. Johnson, C. Ratliff, \& J. Reyman (Ed.), Into the Blogosphere; Rhetoric, Community and Culture of Weblogs. Minnesota: University of Minnesota. Retrieved from http://blog.lib.umn.edu/blogosphere/formation of norms.html.

Wenger, E. (1998a). Communities of Practice: Learning as a Social System. The Systems Thinker, 9(5). Retrieved from http://www.co-i-l.com/coil/knowledgegarden/cop/lss.shtml

Wenger, E. (1998b). Communities of Practice. Learning, Meaning and Identity. Oxford: Cambridge University Press.

Wenger, E., McDermott, R., \& Snyder, W. (2002). Cultivating Communities of Practice. Boston: Harvard Business School Press.

Wiig, K. (2000). Knowledge Management: An Emerging Discipline Rooted in a Long History. In C. Despres \& D. Chauvel (Eds.), Knowledge Horizons: The Present and the Promise of Knowledge Management (pp. 3-26). Boston: ButterworthHeinemann.

Yamazumi, K., Oshima, J., Itoh, D., \& Shimada, M. (2007). Collaborative and Networked Expertise: An Activity-theoretical Study of New Forms of Preservice Teachers' Expansive Learning Kansai: Center for Human Activity Theory, Kansai University

Yin, R. (2003). Case Study Research, Design and Methods (3rd ed.). Newbury Park: Sage.

Yoo, W., Suh, K., \& Lee, M. (2002). Exploring Factors Enhancing Member Participation in Virtual Communities. Journal of Global Information Management 10(3), 5571.

Zhang, W., \& Watts, S. (2003). Knowledge Adoption in Online Communities of Practice. ICIS 2003 Proceedings. Paper 9. Available from http://aisel.aisnet.org/icis2003/9

Zhang, Z. (2009). Personalising Orgnisational Knowledge and Organisationalising Personal Knowledge. Online Information Review, 33 (2), 237-256. 


\section{Appendix A}

\section{A.1 Project initiation documentation}

\begin{tabular}{|c|c|c|c|}
\hline \multirow[b]{2}{*}{18 December 2006} & & \multicolumn{2}{|c|}{ 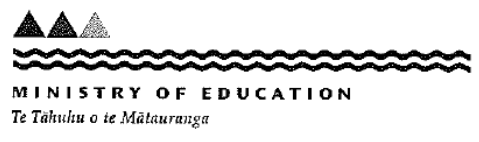 } \\
\hline & National Office & $\begin{array}{l}\text { 45-47 Pipitea Street } \\
\text { Thorndon } \\
\text { PO Box } 1666 \\
\text { Wellington } \\
\text { New Zealand }\end{array}$ & $\begin{array}{l}\text { Phone: } 0-4-4638000 \\
\text { Fax: 0-4-463 } 8001 \\
\text { www.minedu.govt.nz }\end{array}$ \\
\hline
\end{tabular}

Associate Professor Pak Yoong

School of Information Management

Victoria University of Wellington

P.O.Box 600

WELLINGTON

Dear Pak

\section{Confirmation of approval for research into the Ministry's ICT PD programme}

This letter is to confirm the Ministry of Education's approval for Jocelyn Cranefield to undertake student research within the Ministry's Information and Communication Professional Development (ICT PD) Programme.

You and Jocelyn and I have recently met to discuss the Ministry's interest in participating in her student research project entitled "The role of online Communities of Practice in the transfer and embedding of effective teaching practice". Jocelyn has since provided me with a short topic proposal and details of the objectives and scope of her research. The Ministry has agreed to assist Jocelyn in gaining access to participants in the ICT PD Programme for the purposes of this research. I also want to express support for her work as it will be particularly useful in our strategic plans for the use of online environments.

I understand that the research is subject to the University's formal approval process, including presentation of the research proposal. This letter is provided in support of Jocelyn's application for the Human Ethics Committee approval.

Yours sincerely

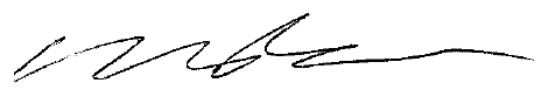

Murray Brown

Manager

e-Learning Unit

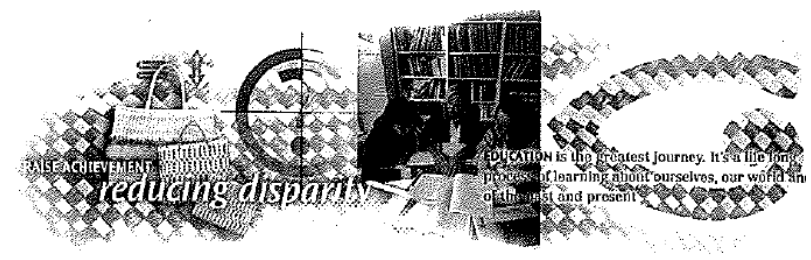




\section{ETHICAL APPROVAL FOR INTERVIEW: INFORMATION SHEET}

$<$ Date $>$

Dear $<$ participant $>$,

\section{The role of online communities of practice in the transfer and embedding of effective teaching practice}

This letter follows up our recent conversation/email in which I expressed an interest in interviewing you about how online Communities of Practice (CoPs) help facilitate the transfer and 'embedding' of effective teaching practice. Prior to conducting the proposed interview, Victoria University of Wellington requires that I obtain your written informed consent.

Purpose of Research

The purpose of the research is to identify how online CoPs facilitate the transfer and embedding of professional knowledge. The project also aims to elucidate the process that is involved, and to identify the kinds of technologies, roles, and other factors that contribute to this process. The research is also being undertaken for the purposes of completing the degree of $\mathrm{PhD}$ in Information Systems. A summary of the research findings will be available to participants.

\section{Confidentiality}

All raw data will be kept confidential. The collected, collated and analysed data may be published in case studies, academic journals and/or presented at conferences. Any information and opinions that you provide will not be attributed to you, and you will not be able to be identified in any way. There will be an opportunity for you to review any written notes or transcripts of recorded sessions that result from the interviews, to ensure that material is recorded accurately. Throughout the project, hard copies of data and interview tapes will be kept in a locked cupboard in my office at Victoria University. Electronic files will be stored in password protected files, with access being restricted to myself my $\mathrm{PhD}$ supervisor. The data will be destroyed two years after the conclusion of the project.

Please feel free to contact Dr Pak Yoong on 4635878 or our Head of School (Professor Sid Huff) on 4635819 if you require further information about the informed consent requirement. You may withdraw from this project at any time up until 1 November 2007, and in this case, your data will be immediately destroyed and excluded from the study.

The consent form is attached. It includes a request for permission to tape-record interviews. The interview will last 60-90 minutes. Please could you complete the form, sign it and return it to me in the enclosed stamped and addressed envelope by 20 February 2007. Please contact me if you have any questions. Thank you. 


\section{Consent to Participation in Research}

\section{The role of online communities of practice in the transfer and embedding of effective teaching practice}

[Please mark each box with a $\checkmark$ to indicate agreement]

1. I have been given and have understood an explanation of this research project and the confidentiality conditions

2. I have had an opportunity to ask questions and have them answered to my satisfaction

3. I agree to be interviewed by Jocelyn Cranefield for the purpose of this research, and I consent to the use of my perceptions, experiences, opinions and information in this research provided they are not attributed to me.

4. I understand that all data collected (tapes and interview transcripts) will be destroyed two years after the completion of the project.

5. I understand that I may withdraw from this project at any time up until 1 November 2007, and that in this instance, all data collected (tapes and interview transcripts) will be immediately destroyed and excluded from the study.

6. I would like to receive feedback on this research, in the form of a research summary

7. I confirm that I DO......... DO NOT....... have the approval of my employer to participate in this research project.

8. I agree to have interviews tape-recorded YES.......

NO............

Name:

Signed:

Date: 


\section{A.2 Preliminary interview script}

\section{Introductory Questions}

1. What is your job? What is your role in the ICT PD programme?

2. What online communities (of practice) do you belong to? (Please list/describe them: both formal and informal communities)

3. What tools/ technologies do you use within these communities? (Forums, email, website, newsletter, listserv, blogs, websites, chat...)

4. How often do you use these tools, and what do you use them for?

\section{Knowledge Transfer and Embedding (Individual Level)}

I'm interested in understanding how professional knowledge to do with teaching with ICT is transferred in the online environment, and especially how online communities help to pass on new ideas, so that they're taken up by different people or schools or clusters...

5. What encourages you to take up new ideas? What part do online communities play in this?

6. Please think of an example of a new teaching idea that you've picked up during the ICT PD programme and that you now use in your school. What part did online communities and technologies play in helping you establish this idea? Can you describe the process?

7. Did any other online community (outside the ICT PD programme) play a part in this?

8. Do you use the online medium for 'reflective practice'? (Please describe)

9. Do you pass on your own ideas in online communities? (Please give an example)

10. Thinking of the online communities you mentioned before, what type of knowledge is transferred?

11. How? What sorts of things help this?

12. What problems are there?

\section{Knowledge Transfer and Embedding (School, Cluster, National Community Levels)}

So far we've been talking about you as an individual and what knowledge you've gained. I'm also interested in how online environment helps to pass on knowledge at the level of the school, and the cluster, and the national community.

13. Do online communities play a part in transferring knowledge within the cluster? How does this happen? (Can you give an example?)

14. What challenges are involved?

15. What shared ideas are there at cluster level? How are these shared and represented online?

16. Do online communities play a part transferring knowledge within the school? How does this happen? (Please give an example)

17. What challenges are involved? 
18. What shared ideas are there at school level? How are these shared and represented online?

19. Do online communities help to transfer knowledge within the national community? How does this happen? (Please give an example)

20. What challenges are involved?

21. What shared ideas are there at the national community level? How are these shared and represented online?

22. Have ideas moved from the national community to the level of your school or cluster? How did this happen? (Please give an example)

23. Have ideas from your school or cluster made their way elsewhere within the national community? How did this happen? What challenges were involved?

24. What shared ideas exist at the level of the national community? How are they shared and represented online?

\section{Wrap Up}

25. In terms of the online community environment overall, what has the biggest impact on the transfer and embedding of professional knowledge? What kind of knowledge is transferred most effectively in this way?

\section{Thank you...}

\section{A.3 Project initiation - notice posted on listserv}

\section{Seeking Online Collaborators!}

Do members of your cluster collaborate with colleagues online, sharing ideas about teaching and learning with ICT? Do you use tools such as forums, blogs, wikis, listervs and/or e-mail groups to support professional learning? Jocelyn Cranefield, a researcher at Victoria University, is seeking participants for a study that investigates how online communities of practice support the transfer of professional knowledge. Up to six clusters will be selected to participate in the research.

If teachers in your cluster or school are regularly exchanging ideas online with colleagues (either within, or outside, of the ICT PD programme using any online environment) and may be willing to participate in interviews, please contact Jocelyn directly at Jocelyn.cranefield@vuw.ac.nz. (Confidentiality applies.) 


\section{Appendix B}

\section{Map of early activity (process) and issue (factor) codes}

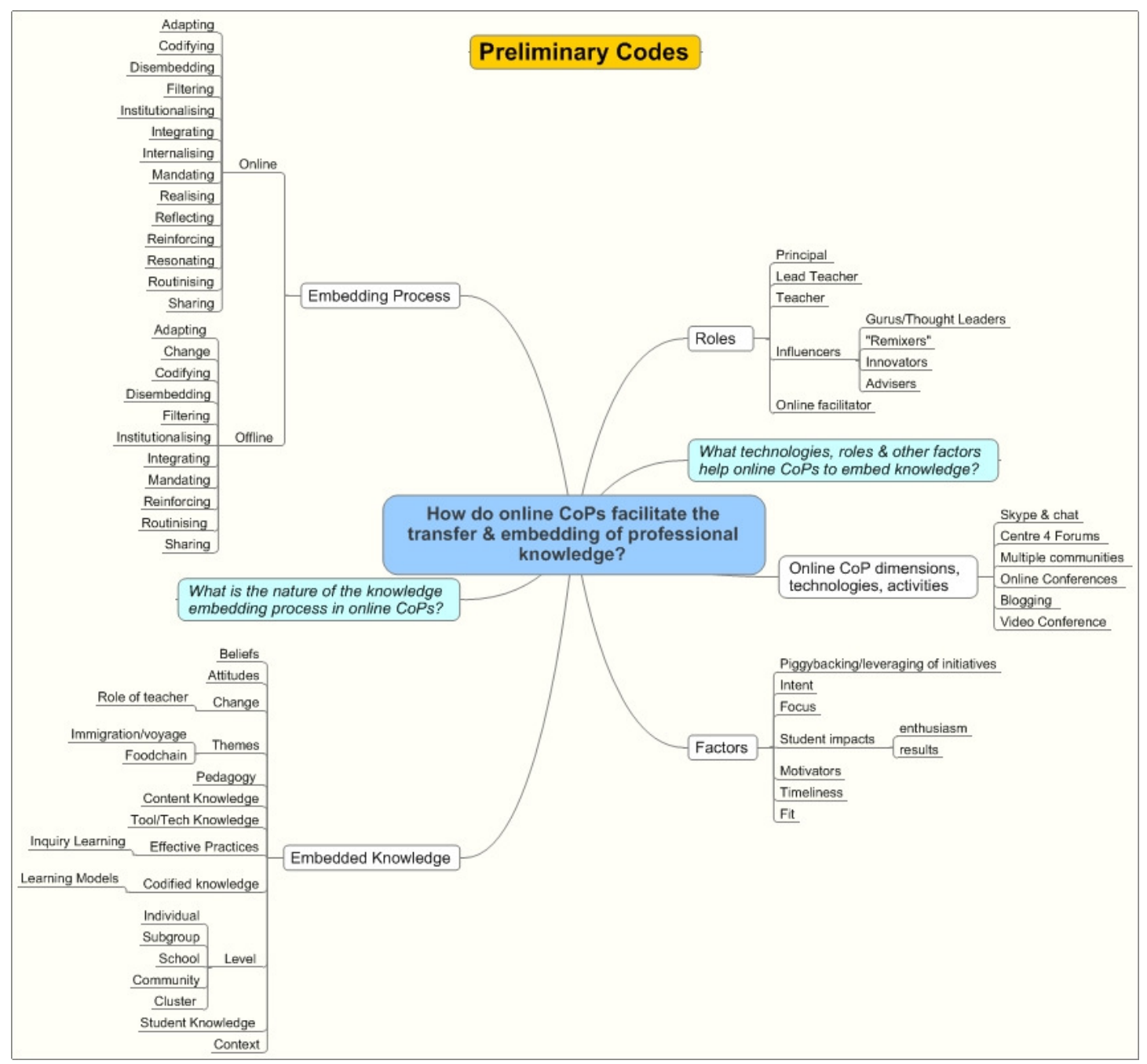




\section{Appendix C}

\section{Feedback wiki: sample pages}

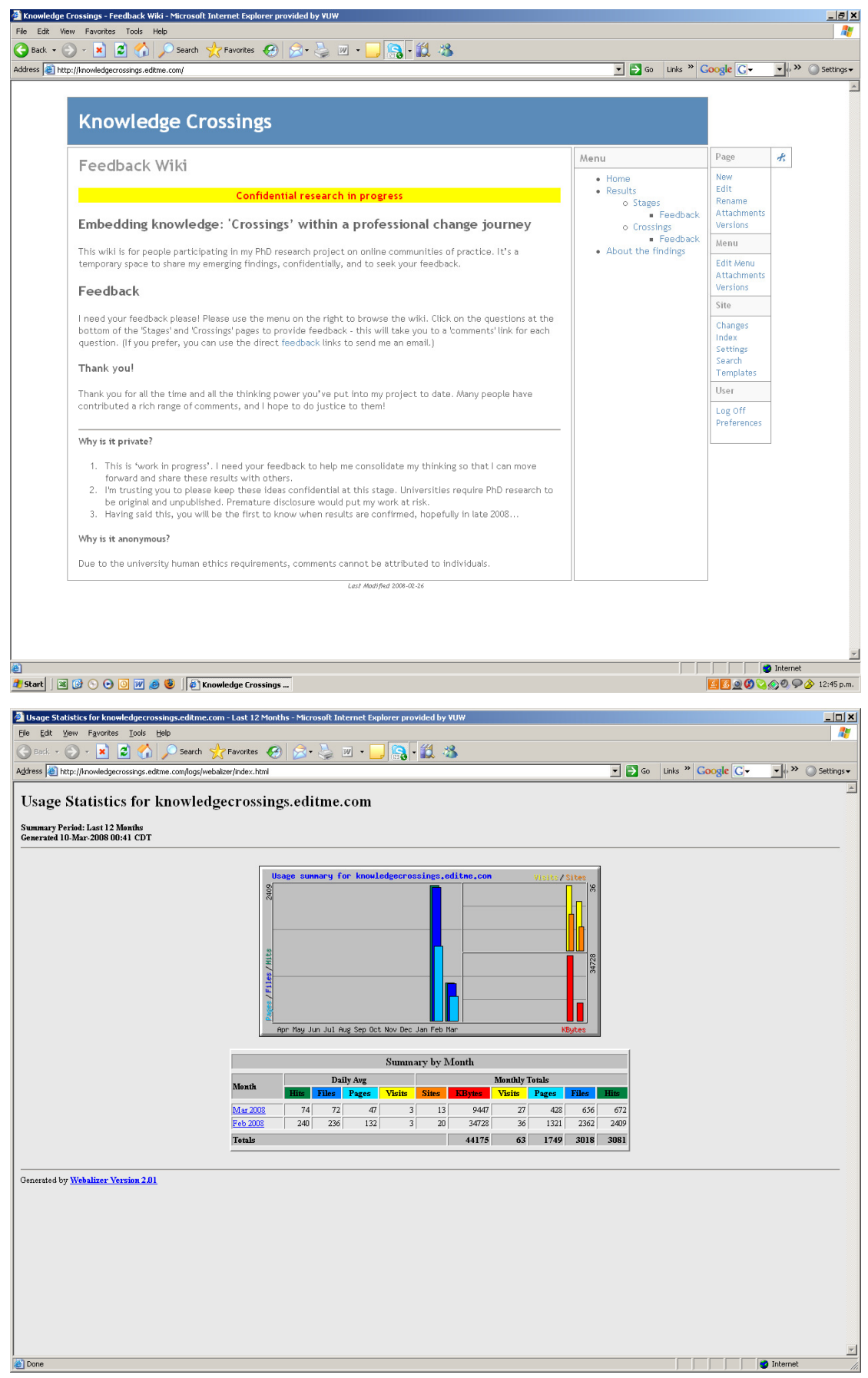




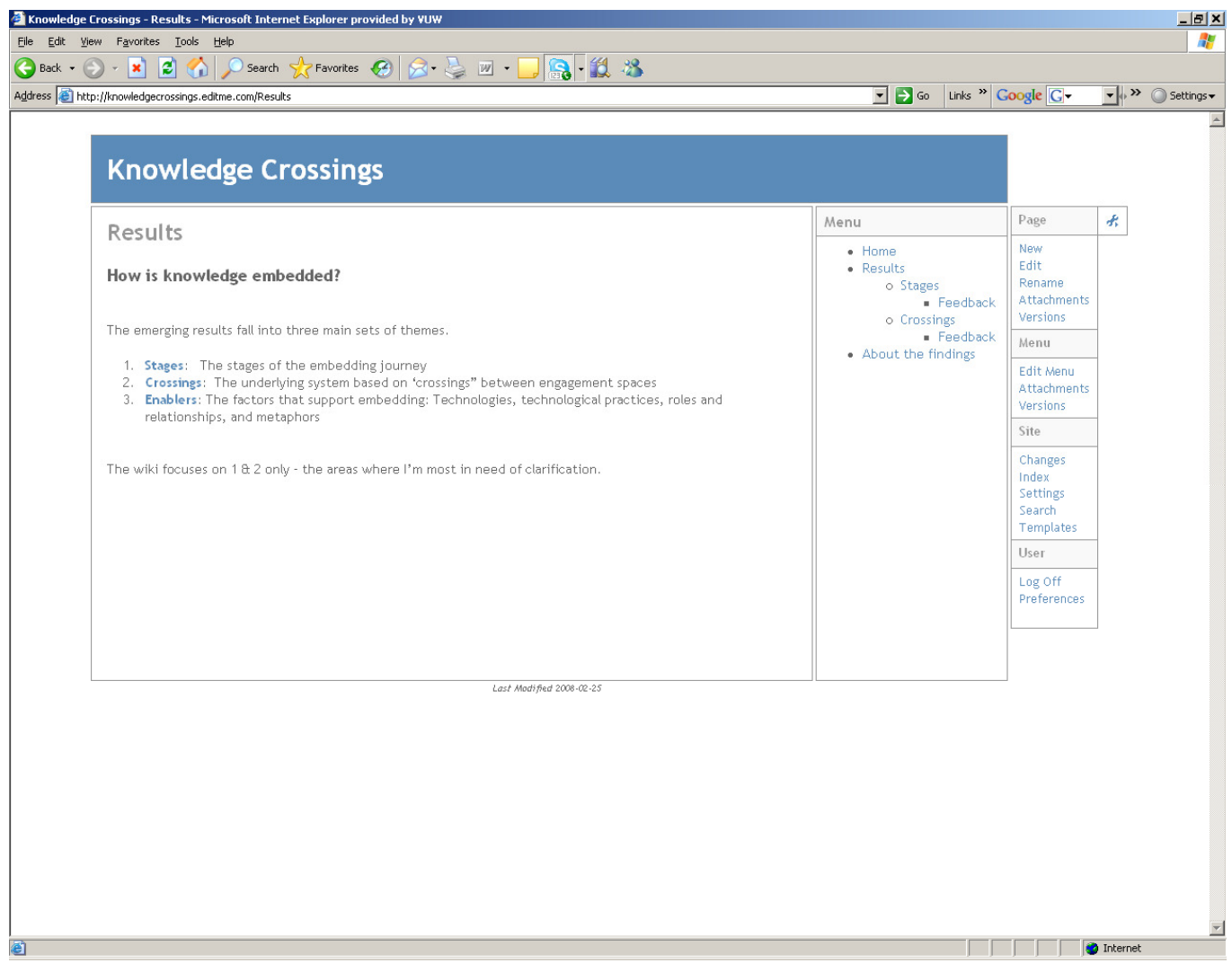

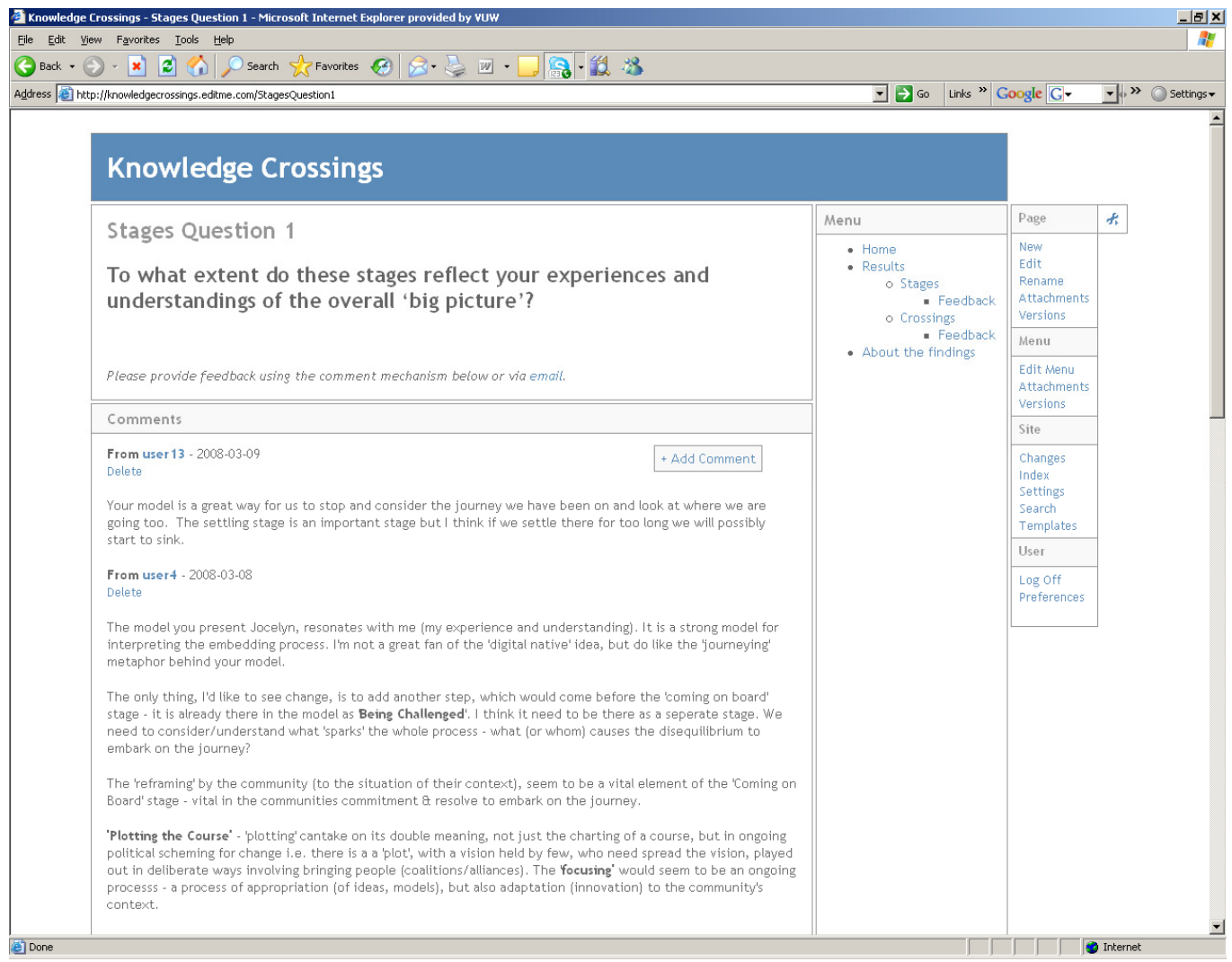



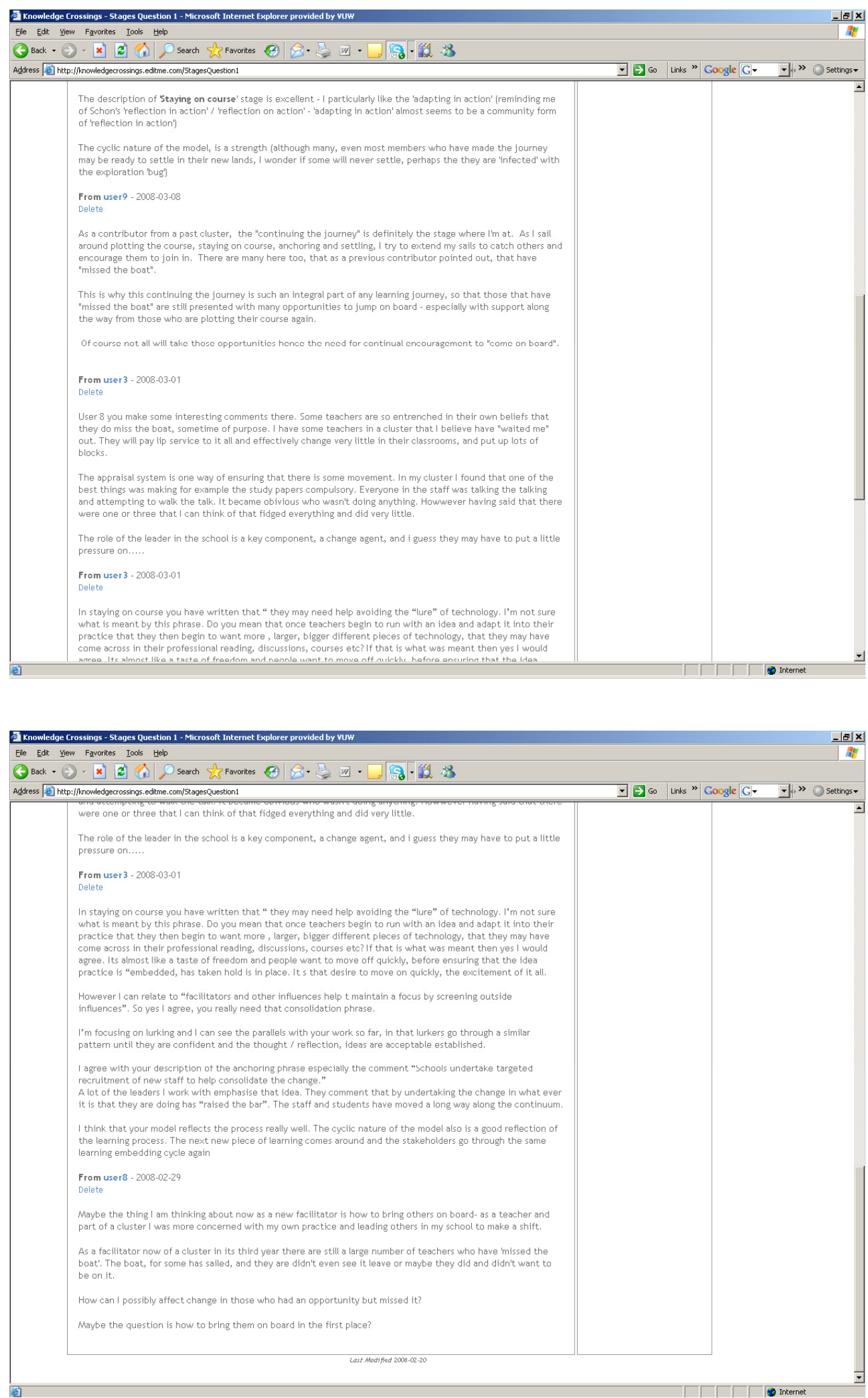


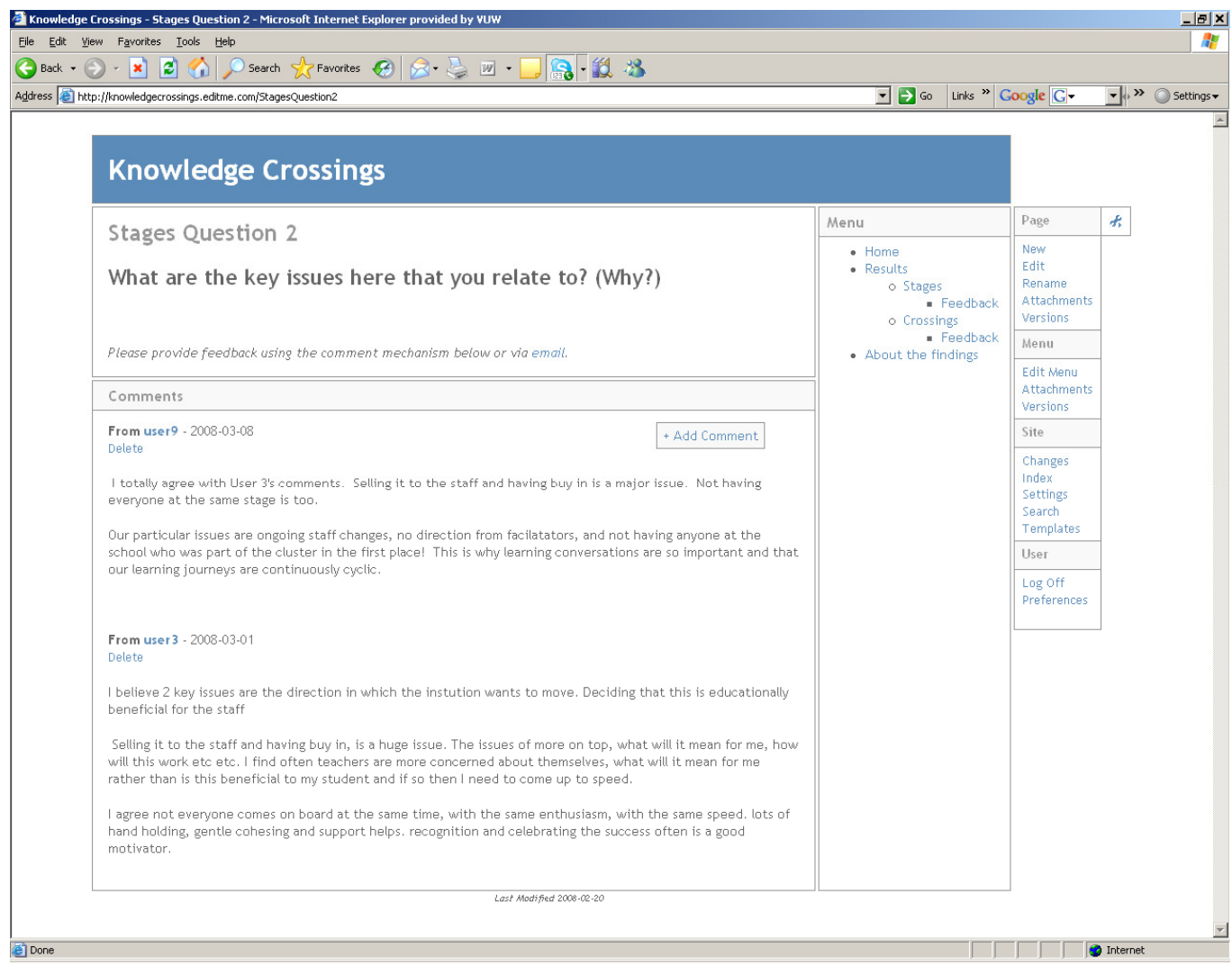

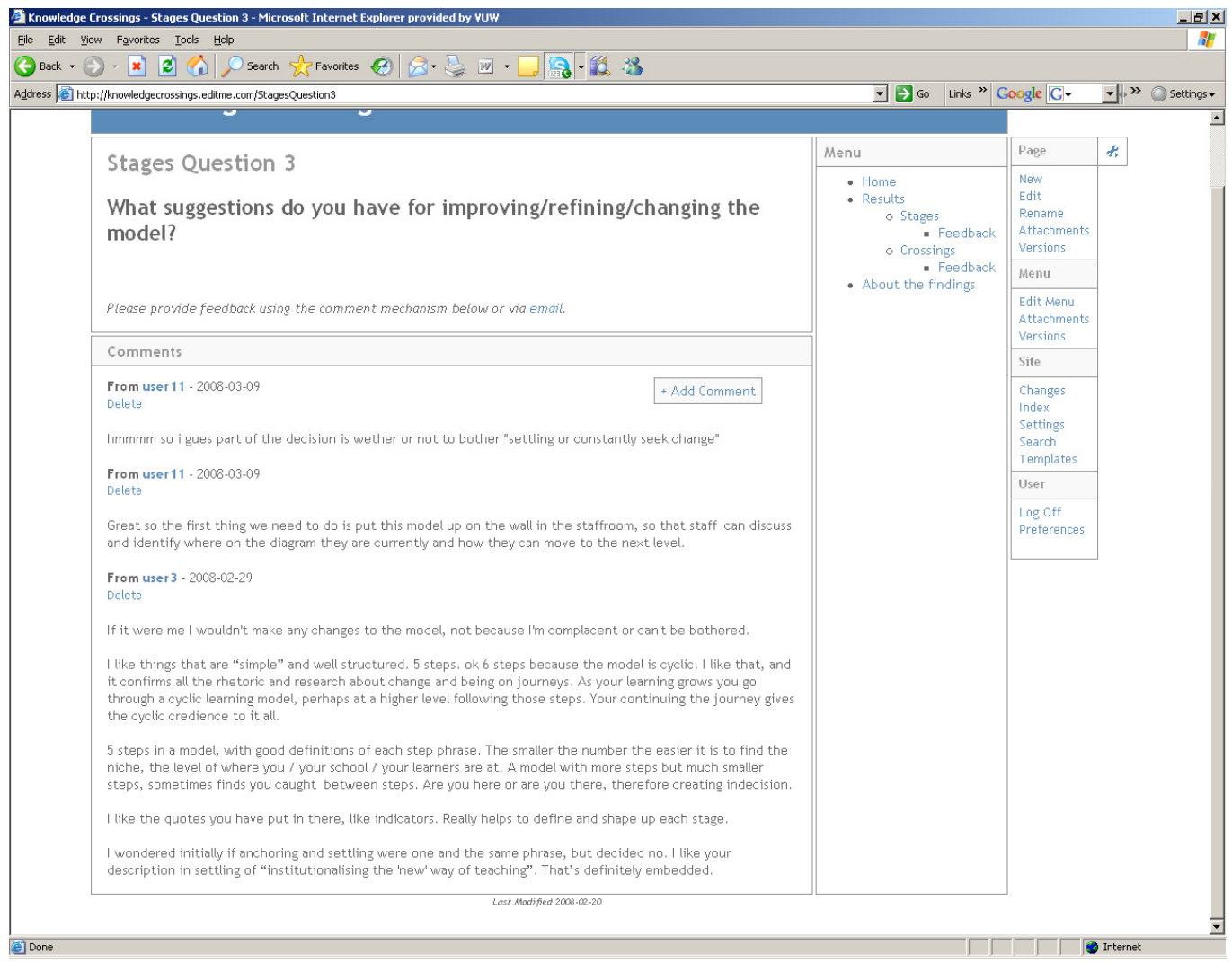



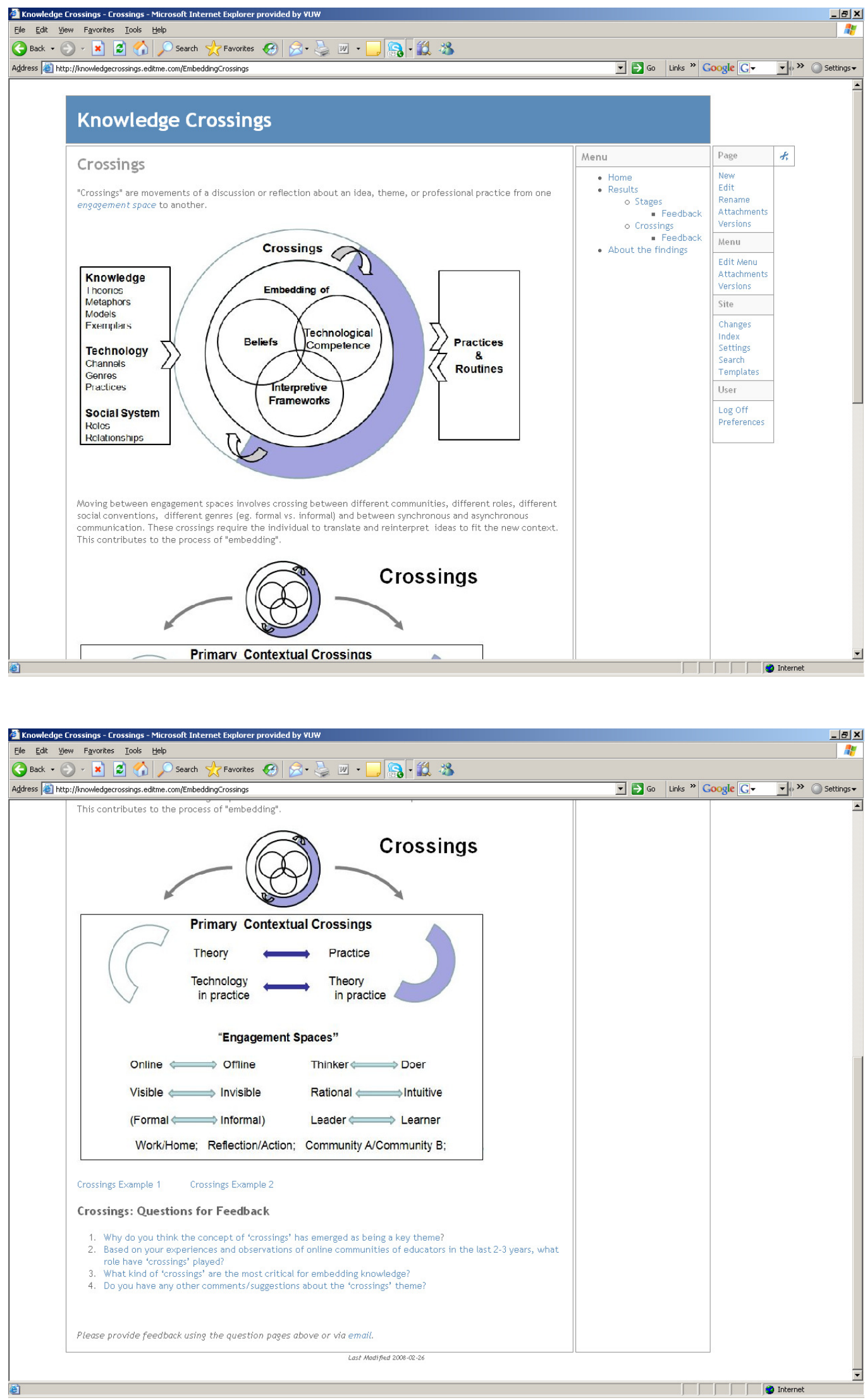


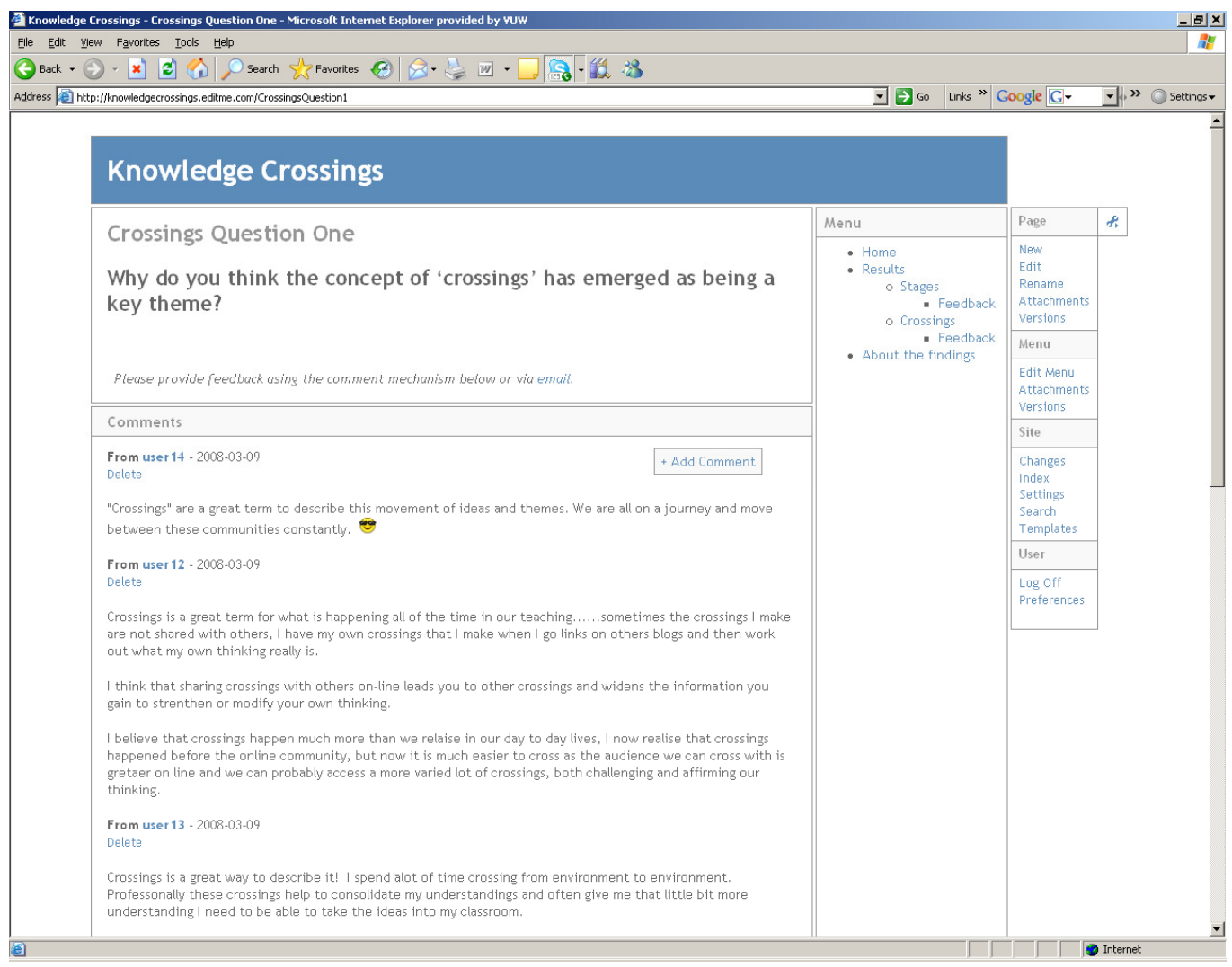

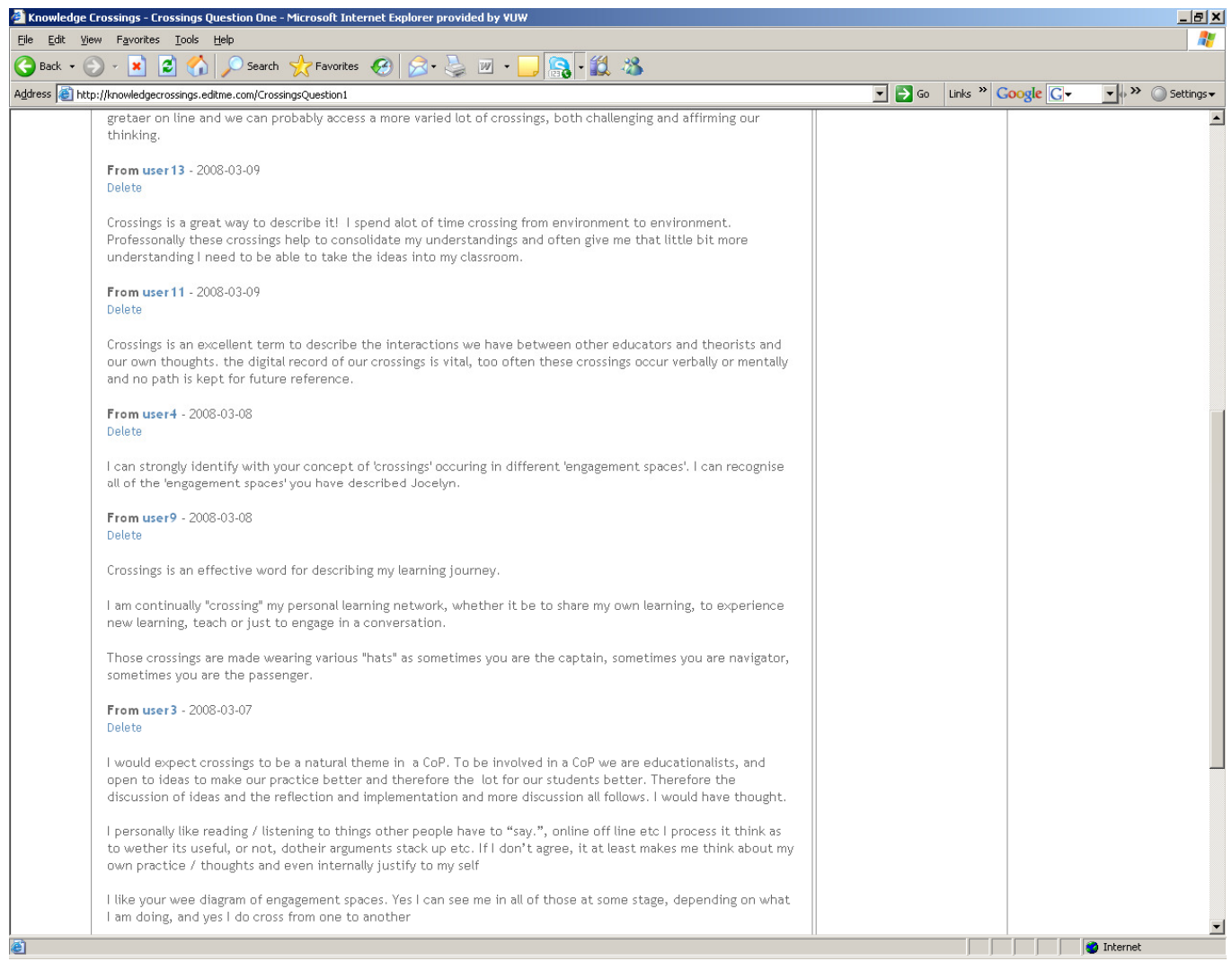



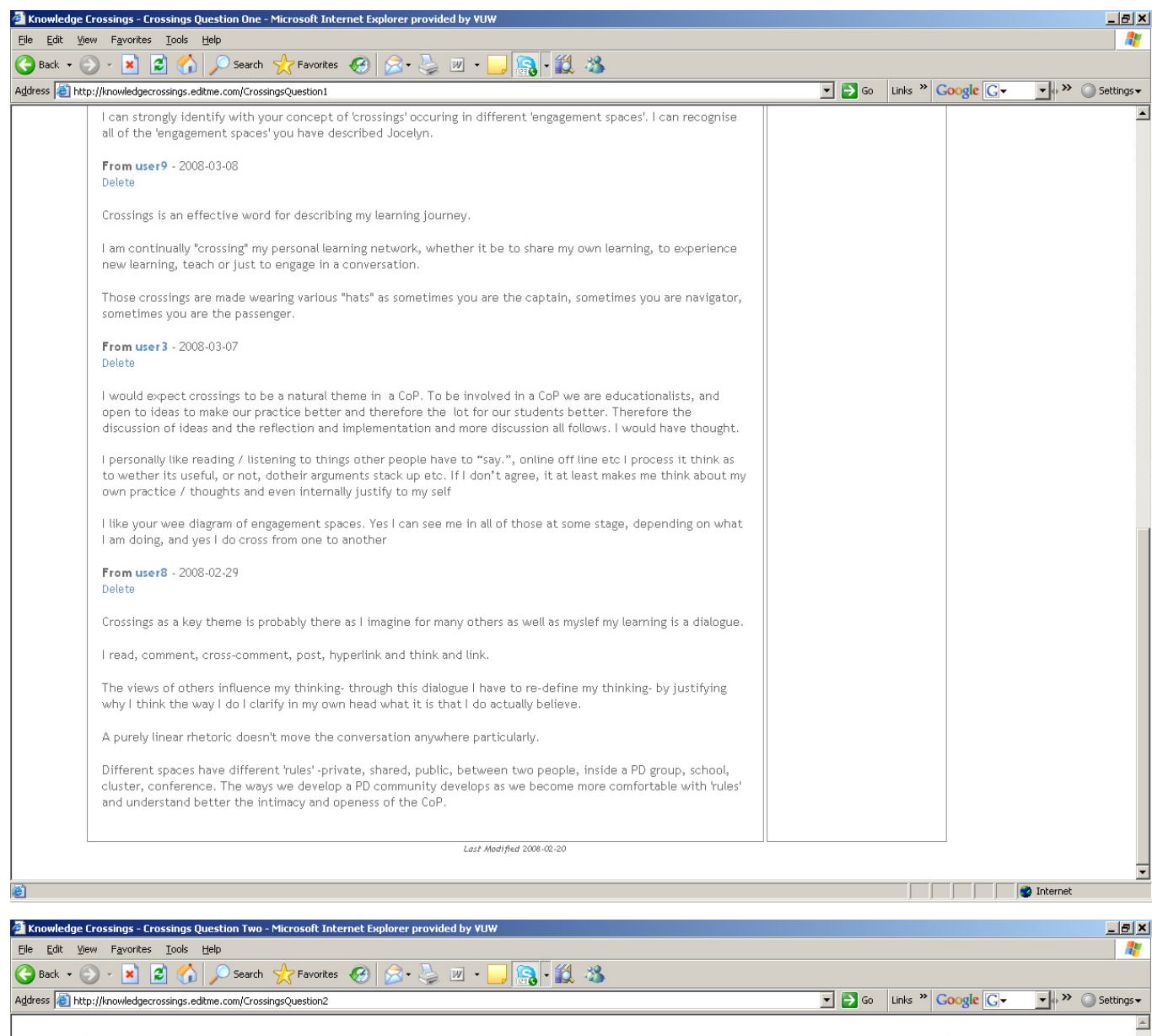

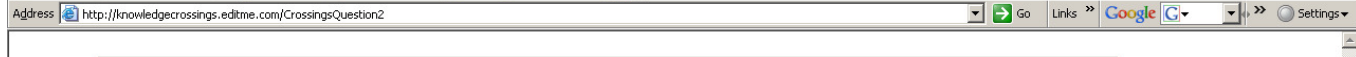

\section{Knowledge Crossings}

Crossings Question Two

Based on your experiences and observations of online communities of educators in the last 2-3 years, what role have 'crossings' played?

Please provide feed back using the comment mechanism below or via email.

Comments

From user $3-2008-03-07$

Delete

+ Add Comment

I'm not sure if this is the place for this quote, but I think its a really good example of a crossing that happened in our online community, and again reinforces my thoughts on crossing reflections and changing our practice. Read reflect chnage practice, read some more, reflect some more.

gain crossing from theopry to practice to thinker to doer etc

Tam amazed at how many sensible comments have been made regarding the use of Clipart. donat think i had ever seriously considered the implications of "quick click" technology before.

Jamie Mckenzie struck a chord unth me by mentioning the cheapening and antution of the authenticity of experience through virtual reantya. Who would want to be guilty of that? Sol am promising myself to us

Last Modififed 2006-00.20

\begin{tabular}{|c|c|c|}
\hline Menu & Page & A \\
\hline \multirow{7}{*}{$\begin{array}{l}\text { - Home } \\
\text { - Results } \\
\text { o Stages } \\
\text { o Feedback } \\
\text { o Crossings } \\
\text { - About the findings }\end{array}$} & $\begin{array}{l}\text { New } \\
\text { Edit } \\
\text { Rename } \\
\text { Attachments } \\
\text { Versions }\end{array}$ & \\
\hline & Menu & \\
\hline & $\begin{array}{l}\text { Edit Menu } \\
\text { Attachments } \\
\text { Versions }\end{array}$ & \\
\hline & Site & \\
\hline & $\begin{array}{l}\text { Changes } \\
\text { Index } \\
\text { Settings } \\
\text { Search } \\
\text { Templates }\end{array}$ & \\
\hline & User & \\
\hline & $\begin{array}{l}\text { Log off } \\
\text { Preferences }\end{array}$ & \\
\hline & & \\
\hline & & \\
\hline
\end{tabular}



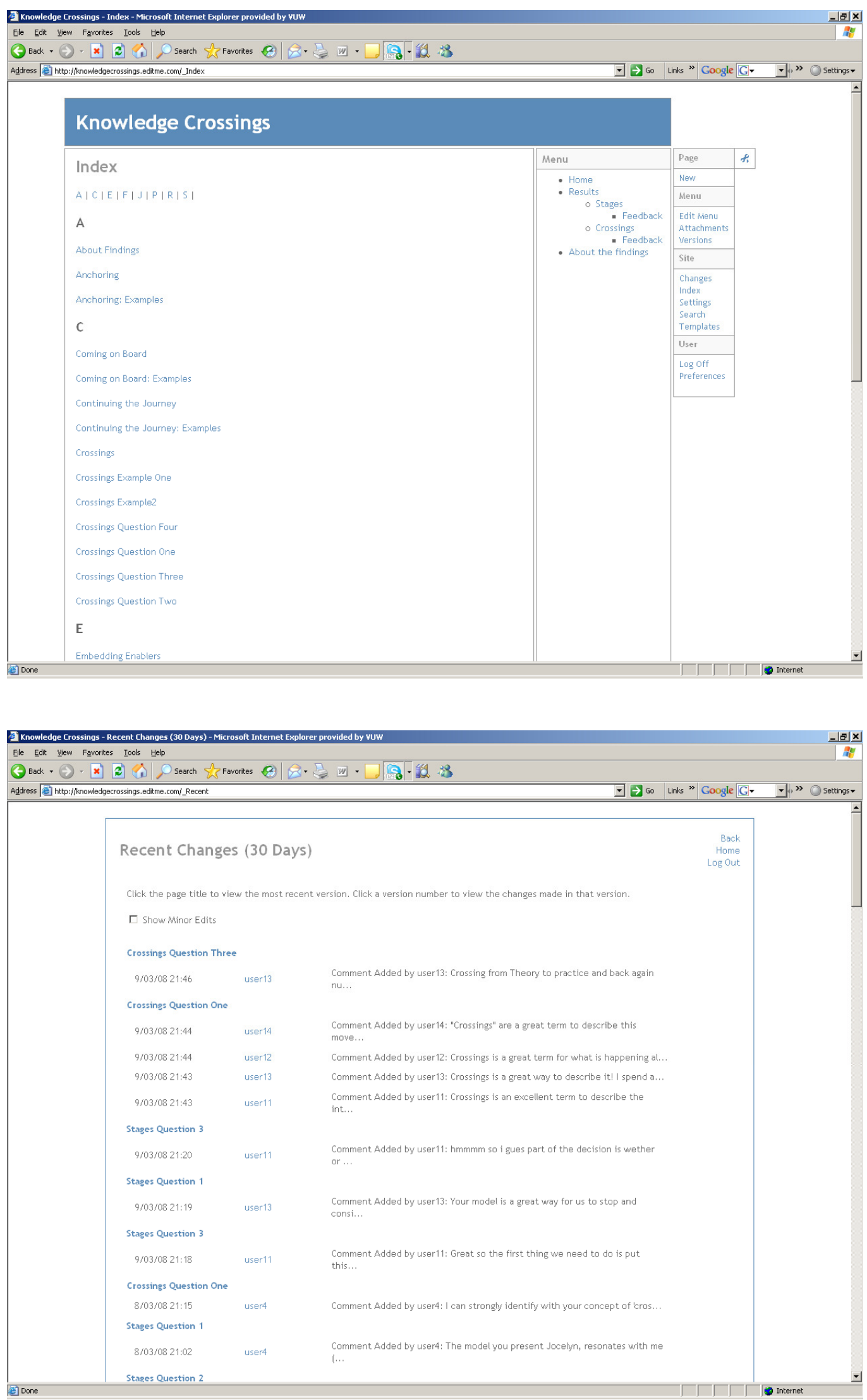


\section{Appendix D}

\section{Presentation slide (Ministry of Education)}

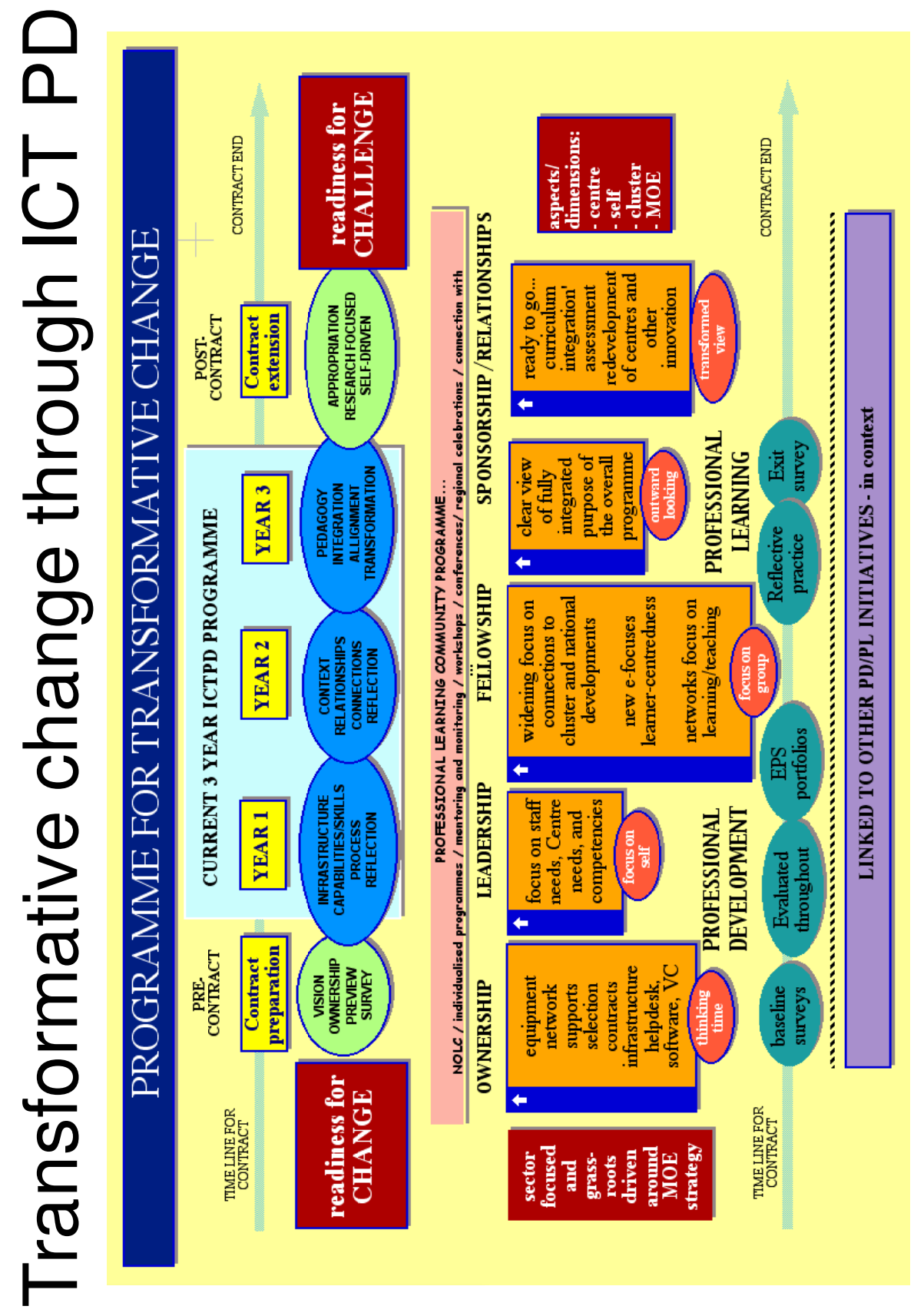




\begin{tabular}{|c|c|c|c|c|}
\hline CoP & Student-Centred Learning & Teacher as Facilitator & Learning Process \& Skills & $\begin{array}{l}\text { Technology to Support Student } \\
\text { Learning }\end{array}$ \\
\hline A & $\begin{array}{l}\text { "In relation to teaching and learning, my belief is that teachers, or we } \\
\text { as a school, have found we are more about individual learning, and } \\
\text { that the learning process, and providing teaching strategies to } \\
\text { support the individual kids".(Principal, interview) }\end{array}$ & $\begin{array}{l}\text { "I step back and listen to the children... allow others to } \\
\text { share their understanding" (Teacher, Online Forum) }\end{array}$ & $\begin{array}{l}\text { "Lest (sic) keep the focus on the learning not the tool. } \\
\text { Tools will constantly change (but)...the skills associated } \\
\text { with learning and thinking will always remain." } \\
\text { (Teacher, Online Forum) }\end{array}$ & $\begin{array}{l}\text { "I am adapting my teaching by always thinking about } \\
\text { when and how technology can be integrated into my } \\
\text { teaching practice to enhance learning" (Teacher, Online } \\
\text { Forum) }\end{array}$ \\
\hline B & $\begin{array}{l}\text { "And they've personal ownership of what they're learning. So } \\
\text { perhaps it's more child-centred, less teacher-directed, and learning } \\
\text { together" (Teacher, interview) }\end{array}$ & $\begin{array}{l}\text { "We group the children into groups of three, and we } \\
\text { basically just check in with each group as the process } \\
\text { goes along, and provide a bit of scaffolding where } \\
\text { necessary...the teacher is no longer seen as the body of } \\
\text { knowledge within the classroom." (Teacher, Interview) }\end{array}$ & $\begin{array}{l}\text { We're teaching the children skills to find the answers, } \\
\text { we're not saying "This is the answer". And they're using } \\
\text { the ICT as a vehicle, not just the library. (Teacher, } \\
\text { interview) }\end{array}$ & $\begin{array}{l}\text { "We've now got groups of three and four computers being } \\
\text { used within classrooms, within reading programmes, } \\
\text { within maths programmes, within other areas of } \\
\text { curriculums, suited to a group of children rather than the } \\
\text { whole class instruction. Teachers are now comfortable to } \\
\text { use ICT as a tool to enhance learning as a part of every day } \\
\text { teaching..." (Principal, interview). }\end{array}$ \\
\hline C & $\begin{array}{l}\text { "...there's a change in perspective about how you view students, how } \\
\text { students learn - and those are quite powerful things, really. And also } \\
\text { a change in perspective about pigeon-holing students. You have to be } \\
\text { more open-minded to their own individual needs." (Teacher, } \\
\text { interview) }\end{array}$ & $\begin{array}{l}\text { "It's become less standing up the front talking and more } \\
\text { sitting alongside facilitating the students learning" } \\
\text { (Teacher, interview) }\end{array}$ & $\begin{array}{l}\text { "Last year we had a school goal that every student gets } \\
\text { sixty credits or more, and as I said, we just about } \\
\text { achieved that completely. This year it's slightly deeper. } \\
\text { It's now looking at developing the thinking skills with all } \\
\text { students, so in a way it's working on that previous year's } \\
\text { target, and actually a quiet goal will be to make sure } \\
\text { every student gets eighty credits as well..." (Teacher, } \\
\text { interview). }\end{array}$ & $\begin{array}{l}\text { "...there's a much more open-minded attitude towards IT } \\
\text { within the school and towards integrating it into the } \\
\text { students' learning .." (Teacher, interview) }\end{array}$ \\
\hline D & $\mathrm{N} / \mathrm{A}$ & N/A & $\mathrm{N} / \mathrm{A}$ & $\mathrm{N} / \mathrm{A}$ \\
\hline $\mathbf{E}$ & $\begin{array}{l}\text { "By the time my class (are) ready for 'work' the oldest Net-Gen will be } \\
38 \text { and filling the jobs... What will that mean for my class? They will } \\
\text { have to adapt further, be quicker, smarter and even more determined } \\
\text { than the Net-Gen. How is that going to impact on my teaching today, } \\
\text { tomorrow, next week?! introduced my kids to the concept of a wiki.. } \\
\text { It is basic, but it has them communicating, sharing and presenting } \\
\text { their work online." (Teacher, blog) }\end{array}$ & $\begin{array}{l}\text { "As class discussions developed, talking partners and } \\
\text { group working and responding via the (interactive } \\
\text { whiteboard) board there came a time when I felt I had } \\
\text { to let the pad go. Into the pupils hands. That safety net } \\
\text { for the both my class and myself was gone. They had to } \\
\text { fix the mistakes themselves" (Teacher, blogpost) }\end{array}$ & $\begin{array}{l}\text { "Yes, the future is unclear but if they leave us armed } \\
\text { with the tools, shortcuts, and critical thinking skills then } \\
\text { they will be prepared for a lifetime of learning and } \\
\text { change." (Teacher, blogpost) }\end{array}$ & $\begin{array}{l}\text { "I probably embed the technology now, rather than just } \\
\text { use it as an add-on.... When I do my planning... it's "This is } \\
\text { the subject that I've got to teach", or "this is the area that I } \\
\text { want to get across to my students. How can I do that with } \\
\text { my equipment?" So it's more that. And probably the off- } \\
\text { spin of that has been more collaboration, more global } \\
\text { projects". (Teacher, interview) }\end{array}$ \\
\hline
\end{tabular}

\section{Confirming the Knowledge Focus: Fit between the CoPs and the four subthemes of the new way}




\section{Appendix F}

Finding CoP E: Reciprocal blog and Twitter linkages
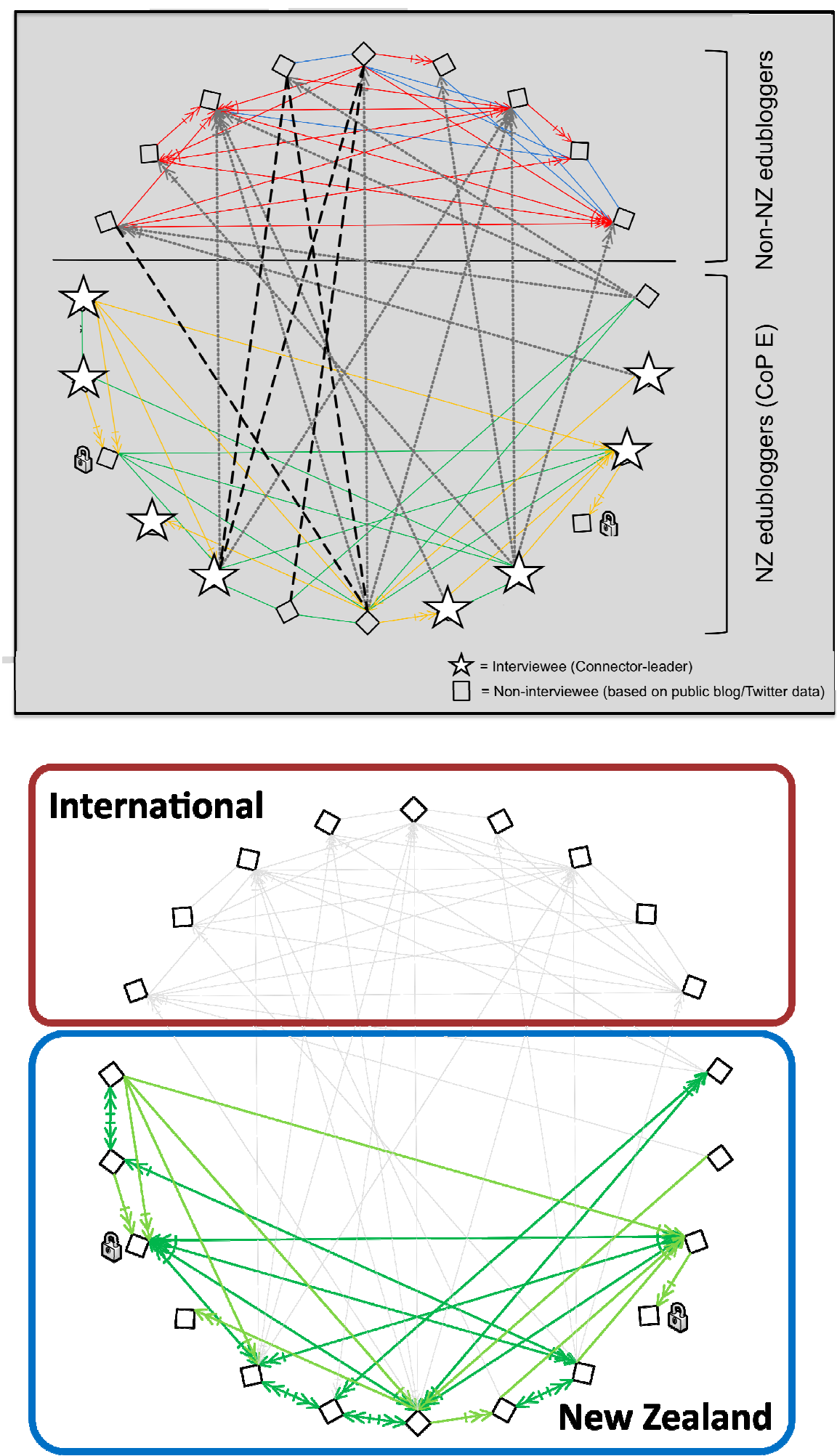


\begin{tabular}{|c|c|c|c|c|c|c|c|}
\hline \multicolumn{2}{|c|}{ Activity } & \multirow{2}{*}{$\begin{array}{l}\text { Description } \\
\text { Reflecting in a way that affirms the direction of change }\end{array}$} & \multirow{2}{*}{ 圛 } & \multirow{2}{*}{ 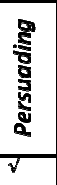 } & \multirow{2}{*}{ 疍 } & \multirow{2}{*}{ 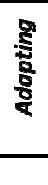 } & \multirow{2}{*}{ s: } \\
\hline 1 & Affirming & & & & & & \\
\hline 2 & Aggregating & Drawing together information and models & V & & 7 & & $\checkmark$ \\
\hline 3 & Benchmarking & Checking one's emerging ideas, impressions, problems and practice against those of others & & & 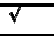 & & \\
\hline 4 & Buddying & Engaging in a sustained professional relationship with a buddy & & $\nabla$ & $\nabla$ & 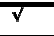 & \\
\hline 5 & Building coalition & Building an alliance to drive and support change & & 7 & $\nabla$ & & v \\
\hline 6 & Building community & Building a sense of community & & & จ & & V \\
\hline 7 & Challenging & Challenging the validity of current practice, causing discomfort and/or tension. This included occasional intervening in practice. & V & $\mathbf{v}$ & & & \\
\hline 8 & Committing & Committing to a change journey (to change, to being open to new knowledge and to working through difficulties) & & $\mathbf{v}$ & & $\nabla$ & $\nabla$ \\
\hline 9 & Consolidating & Consolidating and reinforcing change (e.g. by rolling out an approach, recruiting new staff or setting expectations) & V & & & $\nabla$ & \\
\hline 10 & Contextuallsing & Making changes to practice and/or knowledge to make It better sult a particular context & & & & & $\nabla$ \\
\hline 11 & Customising & Adapting and differentiating a generic model or approach so that it is organisation-specific & & & $\mathbf{\gamma}$ & $\nabla$ & $\nabla$ \\
\hline 12 & Drawing Out & Asking questions that require a deeper level of engagement & $\sqrt{7}$ & & & & \\
\hline 13 & Formalising & Formalising and enshrining a new practice/approach by articulating it via language $\&$ models in shared documentation & V & & $\nabla$ & & $\nabla$ \\
\hline 14 & Going Deeper & Engaging more deeply with the new knowledge and its implications & $\sqrt{7}$ & & & & \\
\hline 15 & Guarding & Protecting teachers from new demands and inputs (to allow catch-up time) & $\mathrm{V}$ & & & v & $\mathbf{v}$ \\
\hline 16 & Having side-conversations & Having conversations in 'invisible' online spaces (such as via IM) with selected members of the online CoP & & V & $\nabla$ & & \\
\hline 17 & Identifying \& aligning with others & Developing a sense of unity with others undergoing change ("we're all in the same boat"); aligning stated views with others & & & r & & \\
\hline 18 & Identifying influencers & Selecting influential people to follow (local/national/ international leaders in practice and/or theory) & & 7 & $\mathrm{q}$ & & \\
\hline 19 & Improvising & Making changes to a practice "on the fly" on the basis of ones' knowledge (c.f. Schön's knowing in practice) & & & & $\mathbf{v}$ & \\
\hline 20 & Institutionalising & Making a new practice and/or approach a mandatory requirement and a norm within the organisation; freezing and guarding it & & & จ & & $\mathbf{v}$ \\
\hline 21 & Intemallsing & Embodylng expllelt knowledge Into more taclt, unconsclous, operatlonal knowledge, vla 'learning by dolng' & & & & $\checkmark$ & V \\
\hline 22 & Leading \& folliowing & Following the Ideas and advice of chosen Influencers and/or provlding this leadershlp to others & & & $\mathrm{r}$ & & \\
\hline 23 & Matching up theory \& practice & Comparing practice \& theory; interpreting \& adjusting practice in light of theory; personalising theory to improve fit & $\mathbf{v}$ & & & $\nabla$ & \\
\hline 24 & Monitoring & Checking on progress against goals and vision; monitoring fit with the new paradigm & V & & $\checkmark$ & & \\
\hline 25 & Personalising & Adapting and differentiating an emerging practice and set of ideas at an individual, personal level & & & & $\checkmark$ & $\sqrt{ }$ \\
\hline 26 & Plggybacking & Building on from the expressed ideas of others, using their language, metaphors etc. & & & $\checkmark$ & & \\
\hline 27 & Piloting & Trying out a new approach over a period and evaluating success & $\mathrm{V}$ & & & $\mathrm{V}$ & \\
\hline 28 & Planning next steps & Planning the next steps in the change journey (changes to make further progress towards the vision) & V & & & & \\
\hline 29 & Problematising & Identifying, thinking about \& articulating practical problems associated with introducing the new knowledge and practice & V & & & & $\mathbf{v}$ \\
\hline 30 & Refining & Making small changes to a newly established model or practice & v & & & $\mathbf{v}$ & \\
\hline 31 & Reframing & Framing the status quo (existing practice) in a way that changes its meaning & $\mathrm{v}$ & $\nabla$ & & $\nabla$ & \\
\hline 32 & Reviewing the journey & Reviewing the professional change process and making sense of it in terms of the (now better understood) new paradigm & V & จ & $\nabla$ & $\nabla$ & $\nabla$ \\
\hline 33 & Routinising & Using a new practice routinely; integrating it into existing routines (and modifying routines as required) & & & & $\nabla$ & \\
\hline 34 & Seeding $(\&$ cultivating) reframing themes & Introducing powerful themes and metaphors that reframe existing practice (and varying them) & $\mathbf{v}$ & $\nabla$ & $\mathrm{T}$ & & \\
\hline 35 & Selecting a focus & Selecting a model or method around which to base change/ embody the new paradigm & $\mathrm{V}$ & & & & \\
\hline 36 & Visioning & Developing a vision for haw the future will be different & 1 & & & & 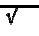 \\
\hline
\end{tabular}




\section{G.2 Activities of the Knowledge Embedding Cycle}

Stage-by-stage breakdown of the KEC showing how different activities promoted the five fundamental embedding processes at each stage (Refer to Appendix G.1 for key to numbering and definition of activities)

\begin{tabular}{|l|l|l|l|l|l|l|}
\hline & $\begin{array}{l}\text { Stage 1. } \\
\text { Plotting the Course }\end{array}$ & $\begin{array}{l}\text { Stage 2. } \\
\text { Coming on Board }\end{array}$ & $\begin{array}{l}\text { Stage 3. } \\
\text { Setting Out }\end{array}$ & $\begin{array}{l}\text { Stage 4. } \\
\text { Staying on Course }\end{array}$ & $\begin{array}{l}\text { Stage 5. } \\
\text { Anchoring }\end{array}$ & $\begin{array}{l}\text { Stage 6. } \\
\text { Settling }\end{array}$ \\
\hline Persuading & 5 & 7,34 & $4,7,34,18$ & 1,4 & $1,4,13$ & 32 \\
\hline focusing & 35,36 & 7,34 & $7,14,23,27,29,34$ & $23,24,28$ & 2,23 & $9,15,30,32$ \\
\hline Aligning & 5 & $6,8,17,34$ & $4,6,18,26,34$ & $3,4,22,24$ & $2,4,13,22,34$ & 20,32 \\
\hline Adapting & & 8,34 & 23,27 & $4,22,23$ & $4,11,19,23,25,33$ & $9,15,21,30,32,33$ \\
\hline Owning & 5,36 & 6,8 & 29, & 10,22 & $2,11,13,25$ & $15,20,21,32$ \\
\hline
\end{tabular}




\section{Appendix $\mathrm{H}$}

A sequence of theory-practice crossings that took place from 2007-2008, tracing the embedding of a role play method of inquiry learning by 'Pete'. Annotations identify the five embedding processes; Focusing (F), Persuading $(P)$, Aligning (Al), Adapting (Ad), and Owning (O).

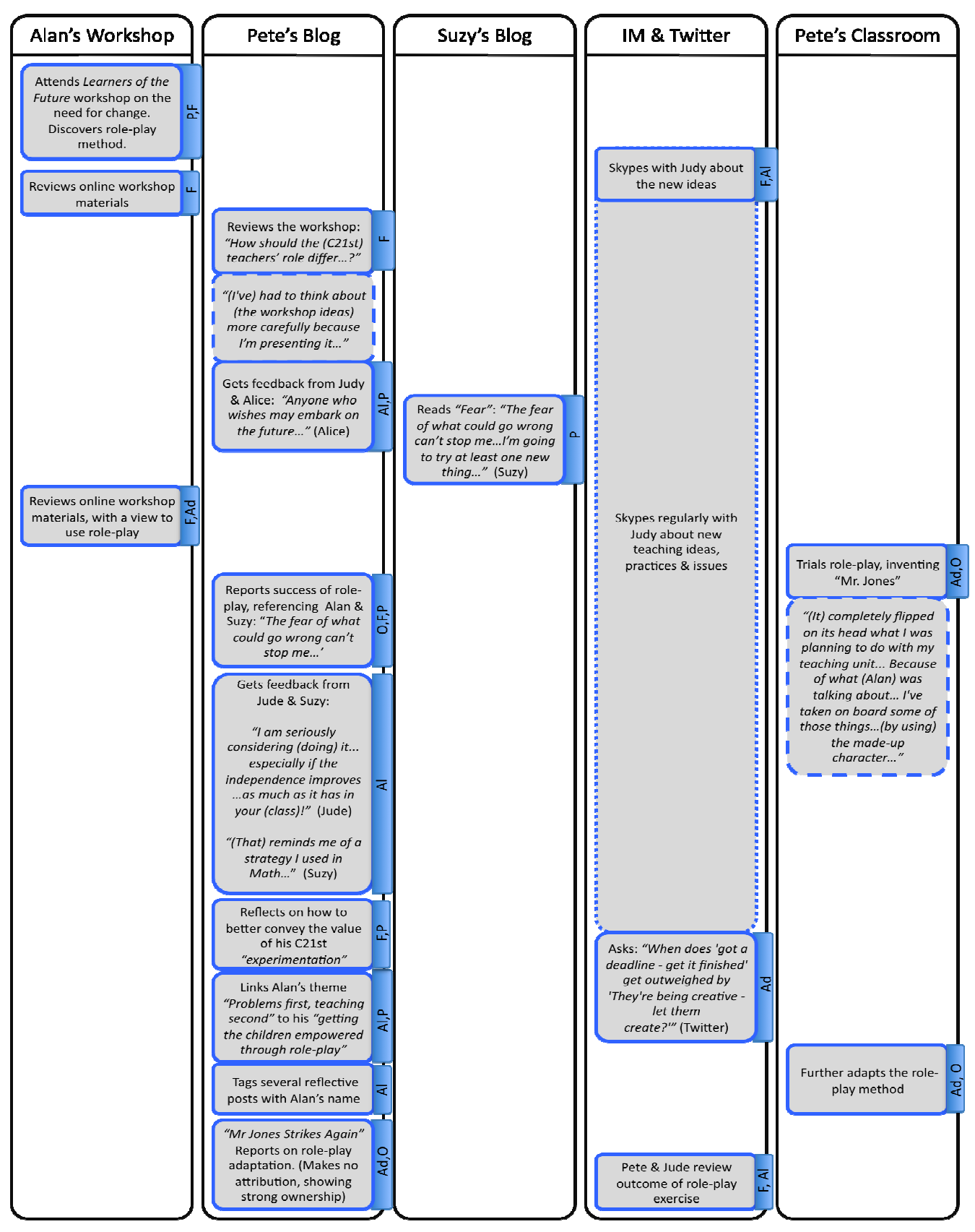

